\title{
IntechOpen
}

\section{Wind Solar Hybrid \\ Renewable Energy System}

Edited by Kenneth Eloghene Okedu, Ahmed Tahour and Abdel Ghani Aissaou 



\section{Wind Solar Hybrid Renewable Energy System}

Edited by Kenneth Eloghene Okedu, Ahmed Tahour and Abdel Ghani Aissaou 

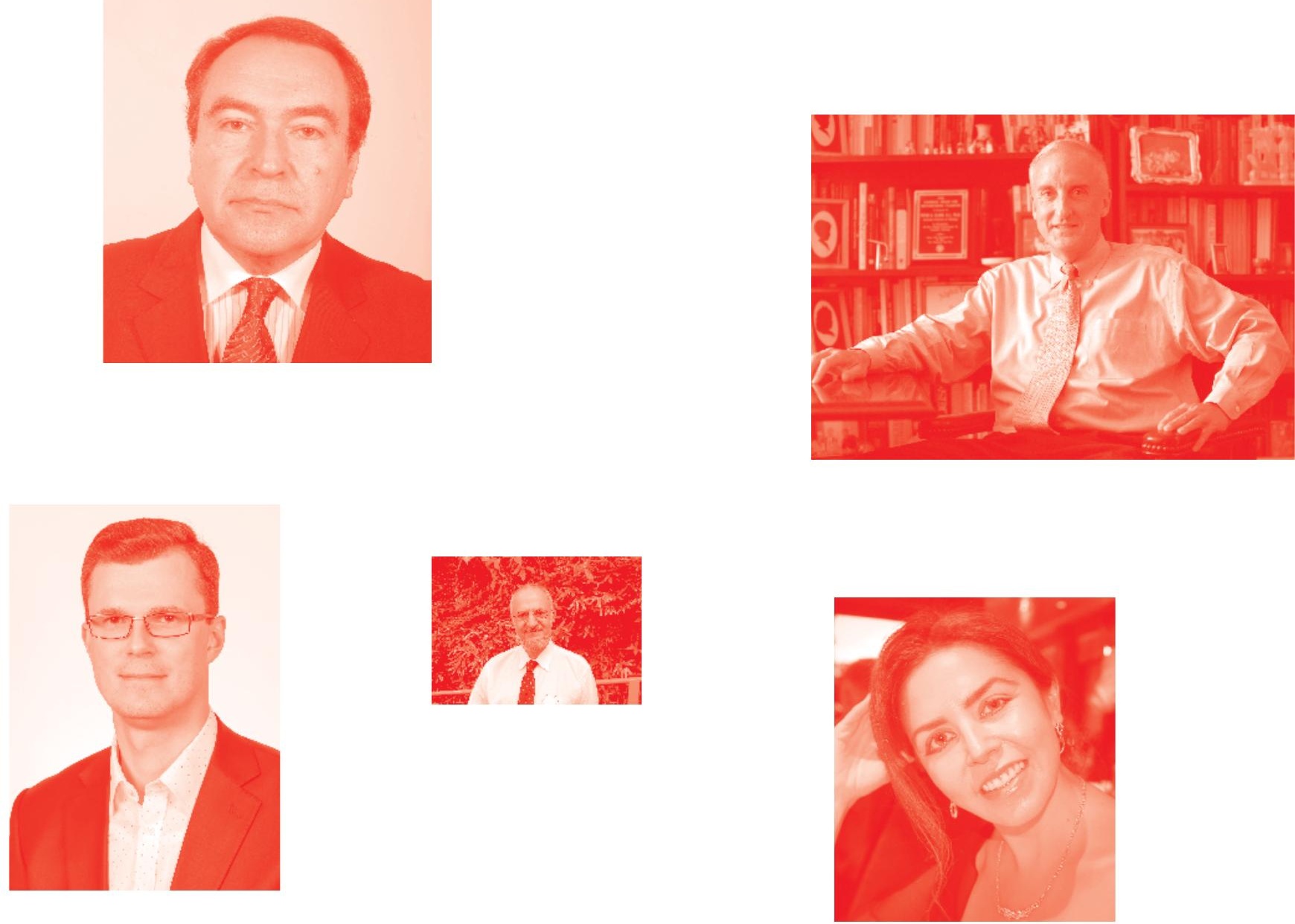

Supporting open minds since 2005
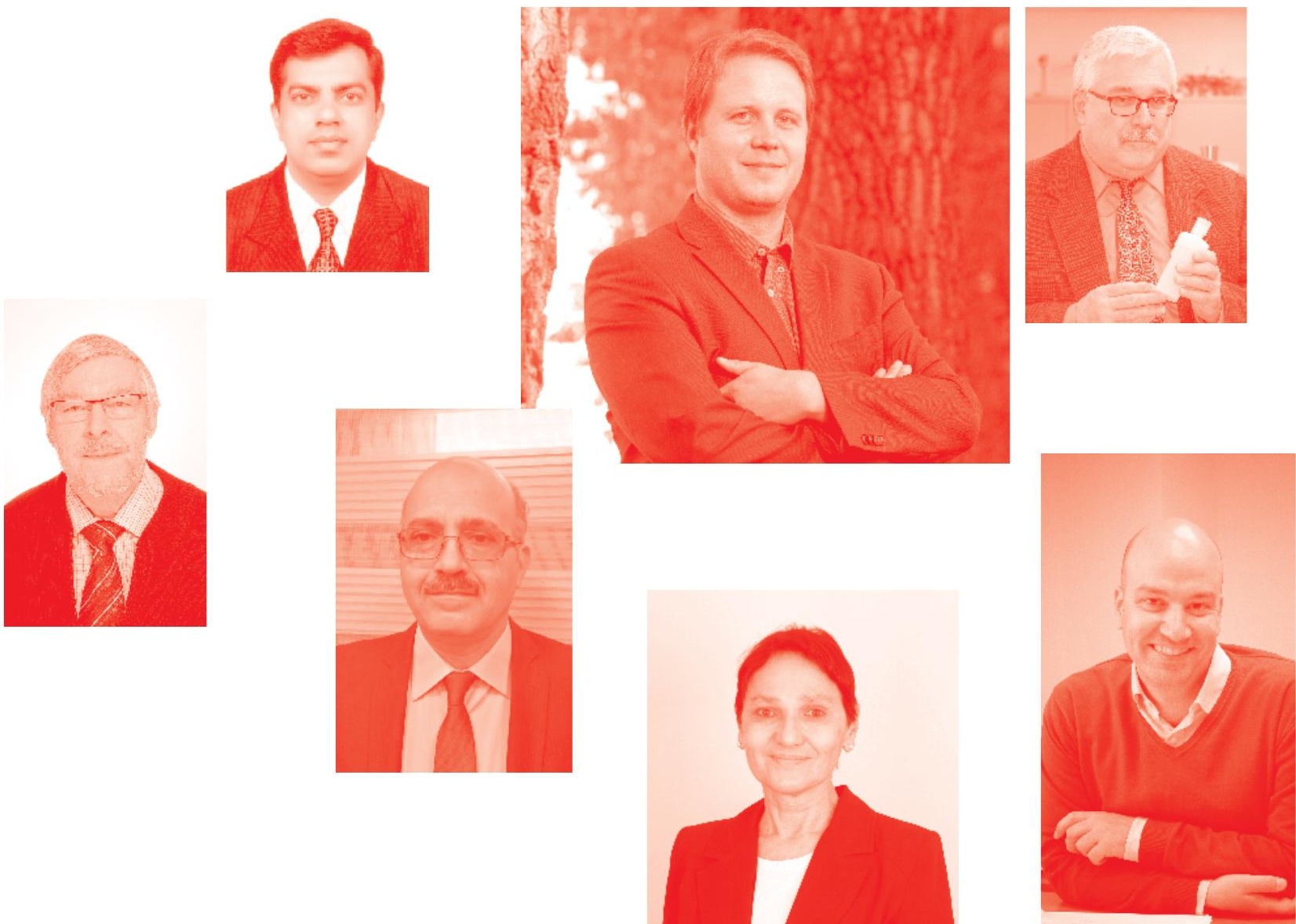
Wind Solar Hybrid Renewable Energy System

http : //dx. doi.org/10.5772/intechopen. 77440

Edited by Kenneth Eloghene Okedu, Ahmed Tahour and Abdel Ghani Aissaou

\section{Contributors}

Weifei Hu, Zhenyu Liu, Jianrong Tan, Alexandre Teplaira Boum, Adolph Moukengue Imano, Steve Alan Talla Ouambo, Gabriel Modukpe, Don Diei, Salisu Muhammad Lawan, Wan Azlan Wan Zainal Abidin, Choon-Man Jang, Sajid Ali, Ochuko Kelvin Overen, Edson Leroy Meyer, Golden Makaka, Sasmita Behera, Bibhuti Bhusan Pati, Jignesh Pravinbhai Patel, Satish Kantilal Joshi, Hongsheng Wang, Nicolás MuñozGaleano, Jesús M Lopez-Lezama, Oswaldo A. Arráez-Cancelliere, Mahesh Kumar

( ) The Editor(s) and the Author(s) 2020

The rights of the editor(s) and the author(s) have been asserted in accordance with the Copyright, Designs and Patents Act 1988. All rights to the book as a whole are reserved by INTECHOPEN LIMITED. The book as a whole (compilation) cannot be reproduced, distributed or used for commercial or non-commercial purposes without INTECHOPEN LIMITED's written permission. Enquiries concerning the use of the book should be directed to INTECHOPEN LIMITED rights and permissions department (permissions@intechopen.com).

Violations are liable to prosecution under the governing Copyright Law .

\section{(cc) BY}

Individual chapters of this publication are distributed under the terms of the Creative Commons Attribution 3.๑ Unported License which permits commercial use, distribution and reproduction of the individual chapters, provided the original author(s) and source publication are appropriately acknowledged. If so indicated, certain images may not be included under the Creative Commons license. In such cases users will need to obtain permission from the license holder to reproduce the material. More details and guidelines concerning content reuse and adaptation can be found at http : //www . intechopen . com/copyright-policy . html .

\section{Notice}

Statements and opinions expressed in the chapters are these of the individual contributors and not necessarily those of the editors or publisher. No responsibility is accepted for the accuracy of information contained in the published chapters. The publisher assumes no responsibility for any damage or injury to persons or property arising out of the use of any materials, instructions, methods or ideas contained in the book.

First published in London, United Kingdom, 2020 by IntechOpen IntechOpen is the global imprint of INTECHOPEN LIMITED, registered in England and Wales, registration number: 11086078 , 7th floor, 10 Lower Thames Street, London,

EC3R 6AF, United Kingdom

Printed in Croatia

British Library Cataloguing-in-Publication Data

A catalogue record for this book is available from the British Library

Additional hard and PDF copies can be obtained from orders@intechopen.com

Wind Solar Hybrid Renewable Energy System

Edited by Kenneth Eloghene Okedu, Ahmed Tahour and Abdel Ghani Aissaou

p. cm.

Print ISBN 978-1-78984-590-7

Online ISBN 978-1-78984-591-4

eBook (PDF) ISBN 978-1-83880-372-8 


\section{We are IntechOpen, \\ the world's leading publisher of Open Access books}

\section{Built by scientists, for scientists}

\section{$4,600+$}

Open access books available

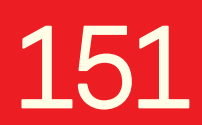

Countries delivered to

\section{$120,000+$}

International authors and editors

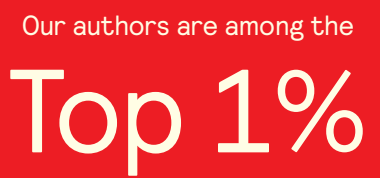

most cited scientists

Contributors from top 500 universities
$135 \mathrm{M}+$

Downloads
1200

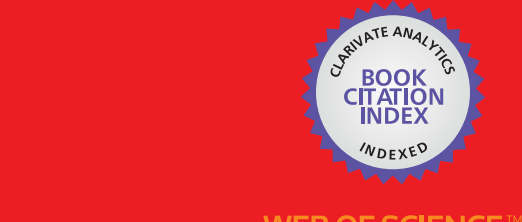

Selection of our books indexed in the Book Citation Index in Web of Science ${ }^{\mathrm{TM}}$ Core Collection (BKCI)

\section{Interested in publishing with us? \\ Contact book.department@intechopen.com}

Numbers displayed above are based on latest data collected.

For more information visit www.intechopen.com 



\section{Meet the editors}

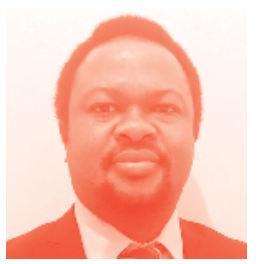

Kenneth Eloghene Okedu was a Massachusetts Institute of Technology (MIT) Research Fellow in the Department of Electrical and Computer Engineering, Cambridge, Boston, USA (2013). He obtained his Ph.D. from the Department of Electrical and Electronic Engineering, Kitami Institute of Technology, Hokkaido, Japan in 2012. He received his B.Sc. and M. Eng. degrees in Electrical and Electronic Engineering from the University of Port Harcourt, Nigeria in 2003 and 2007, respectively, where he was retained as a faculty member from 2005 until the present day. He has also been a visiting faculty member to The Petroleum Institute, (ADNOC), Abu Dhabi. He has also been a visiting faculty member to the Caledonian College of Engineering, Muscat, Oman (Glasgow Caledonian University, UK). He is presently a visiting professor to the National University of Science and Technology (NUST), Muscat, Oman in the Department of Electrical and Computer Engineering. His research interests include power system stability, renewable energy systems, stabilization of wind farms using doubly fed induction generator variable speed wind turbine, augmentation and integration of renewable energy into power systems, grid frequency dynamics, wind energy penetration, FACTS devices and power electronics, renewable energy storage, and hydrogen and fuel cells.

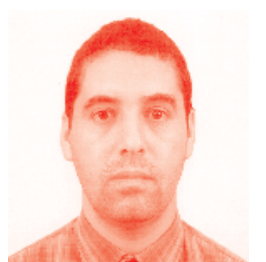

Ahmed Tahour was born in 1972 in Ouled Mimoun, Tlemcen, Algeria. He received his BS degree in Electrical Engineering in 1996, his MS degree in 1999, and his $\mathrm{PhD}$ in 2007 from the Electrical Engineering Institute of the University of Sidi Bel Abbes (Algeria). He is currently Professor of Electrical Engineering at the Superior School of Applied Sciences of Tlemcen (Algeria). He serves as an editor in international journals (IJEAEEE) and as a reviewer in RRST, IJEPES, and CEP (Elsevier), etc. He serves as reviewer and international programme committee member for international conferences: SEB (2014-2019) and ICRERA (2014-2019). His current research interests include power electronics, control of electrical machines, and renewable energies.

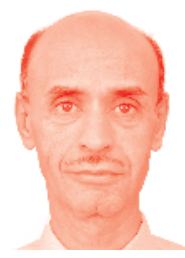

Abdel Ghani Aissaoui is a full Professor of Electrical Engineering at the University of Bechar (Algeria). He was born in 1969 in Naama, Algeria. He received his BS degree in 1993, his MS degree in 1997, and his $\mathrm{PhD}$ degree in 2007 from the Electrical Engineering Institute of Djilali Liabes University of Sidi Bel Abbes (Algeria). He is an active member of the IRECOM (Interaction Réseaux Electriques-COnvertisseurs Machines) Laboratory and an IEEE senior member. He is an editor member and a reviewer for many international journals (IJET, RSE, MER, IJECE, etc. and IJAC, ECPS, COMPEL, etc., respectively).. He serves as a member of a technical committee (TPC) and a reviewer in international conferences (CHUSER 2011, SHUSER 2012, PECON 2012, SAI 2013, SCSE 2013, SDM 2014, SEB 2014, PEMC 2014, PEAM 2014, SEB 2014, 2015, ICRERA 2015, 2016, 2017, 2018, 2019, etc.). His current research interest includes power electronics, control of electrical machines, artificial intelligence, and renewable energies. 



\section{Contents}

Preface

Section 1

Modeling and Control of Wind Turbine

Chapter 1

Thermodynamic Analysis of Wind Energy Systems

by Weifei Hu, Zhenyu Liu and Jianrong Tan

Chapter 2

Advanced Monitoring of Wind Turbine

by Steve Alan Talla Ouambo, Alexandre Teplaira Boum and

Adolphe Moukengue Imano

Chapter 3

Modeling and Simulation of a $10 \mathrm{~kW}$ Wind Energy in the

Coastal Area of Southern Nigeria: Case of Ogoja

by Gabriel Modukpe and Don Diei

Chapter 4

Harmonic Resonance Analysis for Wind Integrated Power System and Optimized Filter Design

by Jignesh Pravinbhai Patel and Satish Kantilal Joshi

\section{Section 2}

Hybrid Technologies: Wind, Solar and Storage Systems

Chapter 5

A Review of Hybrid Renewable Energy Systems Based on Wind and Solar Energy: Modeling, Design and Optimization

by Salisu Muhammad Lawan and Wan Azlan Wan Zainal Abidin

Chapter 6

Grid-Connected Distributed Wind-Photovoltaic Energy

Management: A Review

by Sasmita Behera and Bibhuti Bhusan Pati

Chapter 7

Solar Thermochemical Fuel Generation

by Hongsheng Wang 
Evaluation of PV-Wind Hybrid Energy System for a Small Island by Sajid Ali and Choon-Man Jang

Chapter 9

Methodology for Sizing Hybrid Battery-Backed Power Generation Systems in Off-Grid Areas

by Oswaldo A. Arraez-Cancelliere, Nicolás Muñoz-Galeano and Jesús M. López-Lezama

Section 3

Sustainable Development and Impacts of Renewable Energies

Chapter 10

Towards Sustainable Rural Development in South Africa through Passive Solar Housing Design

by Ochuko K. Overen, Edson L. Meyer and Golden Makaka

Chapter 11

Social, Economic, and Environmental Impacts of Renewable Energy Resources

by Mahesh Kumar 


\section{Preface}

Recently, the fight against polluting emissions, which is the cause of many harmful phenomena like the greenhouse effect, the stratospheric ozone hole, global warming, etc., is on the rise. These harmful gases are emitted mostly by various human activities, thus making the environment unfriendly.

In the field of electricity production, conventional sources of energy based on fossil fuel are considered an important source of pollution. Renewable energies constitute excellent solutions to both the increase in energy consumption and environmental problems. They are clean and present an alternative to meet the needs of today's society. The electrical needs of people can be met through distributed generation provided by renewable energy sources (RES), which reduce the electrical energy generated from conventional sources and therefore their pollutant emissions and consequences.

Renewable energy participation to meet consumer needs is governed by the "Kyoto Protocol," which imposes tangible requirements for the signatory countries. This document provides the basis for the future development of RES. On the basis of the Kyoto Protocol, the European Union requires a strategy for member states, better known as the 20/20/20 strategy. These figures equate to a $20 \%$ reduction in greenhouse gas emissions, $20 \%$ reduction in energy consumption due to energy efficiency, and 20\% participation of RES to meet future objectives. These objectives should be achieved by 2020 .

Despite global development, the use of RES encounters a number of difficulties due to the intermittent availability of these sources. The main difficulty is the lack of guaranteed power from generators, converting primary energy potential into electricity due to the stochastic nature of variations of energy sources. In the case solar energy, the variations are caused by the day/night cycle, clouds crossing the sky, or other obstacles between the sun and the photovoltaic (PV) installations. For wind turbines, the variations in power are due to the variability of wind speed, gusts, and other barriers. In this way, the power of the wind, converted by the wind turbine into mechanical power, has a variable character, because it is proportional to the cube of the wind speed. However, this problem is less pronounced in hydroelectric plants, because the presence of dams that retain the water predetermines the presence of a certain quantity of water. The technological experience acquired form hydraulic turbines facilitates the application of this RES.

Several sources of renewable energy are the subject of great interest for scientific research. In this work, we focus on solar and wind energy. The use of these energies has become competitive due to three main factors:

- They are clean, renewable, and naturally replenished

- The development of the wind turbines and the PV panel industry, 
- The evolution of semiconductor/power electronics technologies and new methodologies for the effective enhancement and control of both variable speed wind turbines and PV systems.

The integration of renewable energy resources into the electric grid is very important, based on recent grid codes that require renewable energy sources to remain grid connected, despite their stochastic nature. A hybrid energy system, including solar power systems and wind turbines, is a very interesting technology. It is more reliable and often yields greater economic and environmental returns than standalone systems (wind, solar, geothermal, etc.).

The goal of this book is to present recent works on the concept, control, and applications in hybrid renewable energy. Developments in this field are rapid, accompanied by many difficulties that require solutions. In this book, different solutions are proposed based on new techniques of control and advanced technology products in the field of hybrid renewable energy.

The book is intended for researchers in the field of design of hybrid renewable energy. For anyone working in the development of renewable energy systems, it is hoped that it will serve as a useful reference work.

This book is divided into three sections:

- In the first section, we present a modeling and simulation of wind turbines and a thermodynamic analysis of wind energy systems. This section consists of four chapters.

- In the second section, a review of hybrid renewable energy is represented, which compares and discusses the state-of-the-art progress of solar thermochemical fuel generation and the characteristics of different systems, which may give technical insight into the development and improvement of solar fuel generation by thermochemical approaches in the future. This section is made up of five chapters.

- In the third section, we present social, economic, and environmental impacts of renewable energy systems. The aim of this study is to analyze the thermal performance of the passive solar house. To this effect, indoor and outdoor conditions of the house, which include air temperature, relative humidity, and solar radiation, are monitored. This section includes two chapters.

The contributions of this book reveal the importance of the subject matter. The aim of this book is to provide new trends of research into renewable energy, and to present recent challenges in the implementation of hybrid renewable energy topologies. We hope that readers will find this book a unique and significant source of knowledge and reference.

Dr. Kenneth E. Okedu

Visiting Professor,

Department of Electrical and Computer Engineering, National University of Science and Technology,

Muscat, Sultanate of Oman 
Dr. Ahmed Tahour

Professor,

Université Mustapha Stambouli de Mascara,

Algeria

Dr. AbdelGhani Aissaoui

Professor,

Electrical Department,

Faculty of Technology,

University Tahri Mohamed of Bechar,

Bechar, Algeria 

Section 1

\section{Modeling and Control of Wind Turbine}





\title{
Thermodynamic Analysis of Wind Energy Systems
}

\author{
Weifei Hu, Zhenyu Liu and Jianrong Tan
}

\begin{abstract}
This chapter studies the efficiency performance of wind energy systems evaluated by energy and exergy analyses. The theories of energy and exergy analyses along with efficiency calculation for horizontal-axis wind turbines (WTs) are provided by a lucid explanation. A $1.5 \mathrm{MW}$ WT is selected for the thermodynamic analysis using reanalyzed meteorological data retrieved from the National Aeronautics and Space Administration's (NASA) Modern-Era Retrospective Analysis for Research and Applications, Version 2 (MERRA-2), data set. Matlab scripts are developed to calculate the energy and exergy efficiencies using the MERRA-2 data set. The energy efficiency presents higher magnitude than the exergy efficiency based on the theoretical derivation and the calculated time series of efficiencies. Comparison of impacts of four meteorological variables (wind speed, pressure, temperature, and humidity ratio) on WT efficiencies shows that although wind speed dominates the turbine's efficiency performance, other meteorological variables also play important roles. In addition, uncertainties of the meteorological variables are represented by the best-fit distributions, which are critically important for evaluating the reliability of wind power performance considering realistic meteorological uncertainty.
\end{abstract}

Keywords: wind energy, thermodynamic analysis, energy efficiency, exergy efficiency, meteorological uncertainty, wind speed, pressure, temperature, humidity

\section{Introduction}

Global installed wind power capacity has been tremendously increased over the last 15 years from 23,900 MW in 2001 to 486,790 MW in 2016 [1]. More than 314,000 WTs are now operating around the world, which accounts for more than $4.3 \%$ of 2015 global electricity demand. Yet it is still far from ambitious targets, e.g., increasing wind energy's contribution to $20 \%$ of US electricity supply by 2030 [2]. To approach that, it is of critical importance to accurately evaluate the WT performance considering realistic environmental conditions.

The most common factors that are considered when planning a wind farm include substantial wind resources, landowner and community support, feasible permitting, compatible land use, nearby access to electrical grid, appropriate site conditions for access during construction and operations, aviation compatibility, and favorable electricity market [3]. However, the influences of meteorological variables (e.g., pressure, temperature, and humidity) are often neglected which 
could cause inaccurate evaluation of WT performance. For example, a dry air assumption (i.e., constant air density) does not really consider the moisture changeability. Baskut et al. discussed the effects of several meteorological variables including air density, pressure difference, humidity, and ambient temperature on exergy efficiency and suggested that neglecting these meteorological variables while planning wind farms could cause important errors in energy calculations [3].

The efficiency performance of a WT can be studied in two aspects, energy and exergy efficiencies. The former is calculated as the ratio of produced electricity to the total wind potential within the swept area of the rotor. Thus, only the kinetic energy of the air flow is considered in the energy efficiency calculation, while other meteorological variables such as pressure and temperature are often neglected. The latter considers the maximum useful work that can be obtained by a system interacting with an environment in thermodynamic equilibrium state [4]. The exergy efficiency along with availability and capacity factor of a small WT (rated power $1.5 \mathrm{~kW}$ ) has been studied in Izmir, Turkey, to assess the WT system performance [5]. Sahin et al. developed an improved approach for the thermodynamic analysis of wind energy using energy and exergy, which provided a physical basis for understanding, refining, and predicting the wind energy variations [6]. According to [7], exergies are suggested as the most appropriate link between the second law of thermodynamics and the environmental impact, in part because it measures the deviation between the states of the system and the environment.

This brief précis thus illustrates the importance of energy and exergy analyses for wind energy systems considering meteorological variables and provides a motivation for the thermodynamic analysis conducted herein. The chapter presents the methods and results of thermodynamic analysis of a 1.5 MW WT, which is assumed to be deployed in the northeastern United States, experiencing meteorological reanalysis data retrieved from the NASA's MERRA-2 data set. Matlab scripts are developed to calculate the energy and exergy efficiencies using the MERRA-2 data set. Section 2 provides the fundamental theory of thermodynamic analysis, particularly in derivations of energy and exergy efficiencies. The studied site, meteorological data, and the selected WT are explained in Section 3, which is followed by results and discussion in Section 4. Concluding remarks are provided in Section 5.

\section{Theory}

A WT converts kinetic energy from air flow to electrical energy through subassemblies including rotor blades, drivetrain, generator, and electronic control systems, as well as other auxiliary components. As the kinetic energy is extracted, the air flow that passes through the turbine rotor must slow down. Assuming there is a boundary surface that contains the affected air flow inside, a long stream tube extended far from the upstream and to the downstream with varied cross sections is often used to study the thermodynamics of horizontal-axis WTs [6, 7] (Figure 1). The wind speed, pressure, and temperature at the inlet of the stream tube are represented by $V_{1}, P_{1}$, and $T_{1}$, respectively. Their counterparts at the outlet are $V_{2}$, $P_{2}$, and $T_{2}$ and at the rotor are $V_{\text {ave }}, P_{\text {ave }}$, and $T_{\text {ave }}$. Here a constant specific humidity ratio is assumed in the stream tube for a short-period time (e.g., 10 minutes or 1 hour). The following sections explain the theory of WT thermodynamics in two aspects, energy analysis and exergy analysis, which both apply the meteorological variables such as wind speed, air density, atmospheric pressure, temperature, and humidity. The use of energy and exergy efficiencies considering a comprehensive set of meteorological variables can enable us to accurately evaluate the efficiency performance of WTs. 


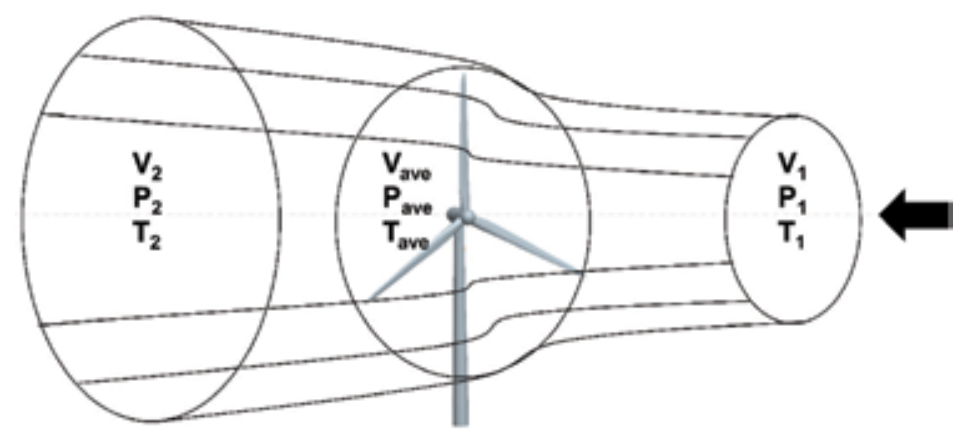

Figure 1.

A schematic plot of WT stream tube for thermodynamic analysis.

\subsection{Energy analysis}

The energy analysis of WT systems stems from the air flow's kinetic energy $E_{k}$ that is calculated as

$$
E_{k}=\frac{1}{2} m V^{2}
$$

where $m$ and $V$ are the mass and speed of the air flow, respectively. The mass $m$ can be further expressed as

$$
m=\rho A V t
$$

where $\rho$ is the air density, $A$ is the rotor swept area perpendicular to the flow, and $t$ is the time that the flow passing through the swept area with speed $V$. By applying the simple momentum theory, the rate of momentum change is equal to the overall change of velocity times the mass flow rate $\dot{m}$, i.e.,

$$
\dot{M}=\dot{m}\left(V_{1}-V_{2}\right)
$$

where $V_{1}$ and $V_{2}$ are the wind speeds at the inlet and outlet, respectively, of the stream tube (Figure 1). The rate of momentum change is also equal to the resulting thrust force. Thus, the power absorbed by the WT is calculated as

$$
P=\dot{m}\left(V_{1}-V_{2}\right) V_{\text {ave }}
$$

where $V_{\text {ave }}$ is the average flow speed at rotor. On the other hand, the rate of kinetic energy change of the flow can be calculated as

$$
\dot{E}_{k}=\frac{1}{2} \dot{m}\left(V_{1}^{2}-V_{2}^{2}\right)
$$

Based on the conservation of energy, Eqs. (4) and (5) should be equal which results in

$$
V_{\text {ave }}=\frac{1}{2}\left(V_{1}+V_{2}\right)
$$

Hence, the retardation of the wind before the rotor $\left(V_{1}-V_{\text {ave }}\right)$ is equal to the retardation of the wind after the rotor $\left(V_{\text {ave }}-V_{2}\right)$. By Eqs. (2), (4), and (6), the rotor power can be calculated as 


$$
P=\frac{1}{4} \rho A\left(V_{1}+V_{2}\right)^{2}\left(V_{1}-V_{2}\right)
$$

Let $a=\frac{V_{2}}{V_{1}}$; Eq. (7) can be reformulated as

$$
P=\frac{1}{4} \rho A V_{1}^{3}(1+a)^{2}(1-a)
$$

In order to obtain the maximum power, equate 0 to the differentiation of Eq. (8) with respect to $a$ resulting in $a=\frac{1}{3}$. Thus, the maximum power $P_{\max }=\frac{8}{27} \rho A V_{1}^{3}$ is achieved, when the outlet wind speed is equal to one-third of the inlet wind speed. Defining the power coefficient as

$$
C_{p}=\frac{P}{\frac{1}{2} \rho A V_{1}^{3}}
$$

the maximum power coefficient is calculated $C_{P_{\max }}=\frac{16}{27} \approx 0.593$. This maximum power coefficient, known as the Lanchester-Betz limit (or Betz limit) [8, 9], explains the maximum power that can be extracted from the air flow and can also be easily derived by other theories (e.g., the rotor disc theory and blade element momentum theory [10]).

Despite the simplicity of Eq. (9) when calculating power coefficient, the total input power in the denominator does not take account of the impacts from pressure, temperature, and humidity. Actually the air density changes as the ambient pressure, temperature, and humidity change, which can be expressed as

$$
\rho=\frac{1+\omega}{R_{a}+\omega R_{v}} \frac{p}{T}
$$

where $\omega(-)$ is the humidity ratio of air, gas constant $R_{a}=287.1 \mathrm{~J} / \mathrm{kg} \mathrm{K}$, water vapor constant $R_{v}=461.5 \mathrm{~J} / \mathrm{kg} \mathrm{K}$, and $T$ is the absolute temperature (unit: $\mathrm{K}$ ). In order to distinguish wind power $P$, the small letter $p$ is used to represent the pressure (unit: $\mathrm{Pa}$ ) in the humid air hereafter. Combining Eqs. (9) and (10), the power coefficient of a WT considering a comprehensive set of meteorological variables can be expressed as

$$
C_{p}=\frac{2\left(R_{a}+\omega R_{v}\right)}{1+\omega} \frac{T P}{p A V_{1}^{3}}
$$

The above derivations provide the fundamentals of the theoretically available energy/power that a WT can extract from the air flow. However, various effects could have influence on the real power output, e.g., vortices shed from the blade tip and hub could significantly affect the rotor lift force and power output [11]. Power losses also occur during the energy transformation through rotor to mechanical shaft and to generator that converts angular kinetic energy to electrical energy. In addition, sustained high wind speeds could cause strong fatigue and extreme loads on WT systems without proper turbine control or safety protection. Thus, wind power is intended to be constrained, when the inflow wind speed is beyond a rated value (i.e., rated wind speed), through different strategies commonly including stall regulation, pitch regulation, and yaw control [12]. As a result, the output power $P_{\text {out }}$ of a WT is corresponding to four operating stages: (1) zero power when the inflow wind speed is smaller than a cut-in wind speed, (2) exponentially increased power as the wind speed increases between the cut-in wind speed and the rated wind speed, (3) rated output power when the wind speed is between the rated wind 


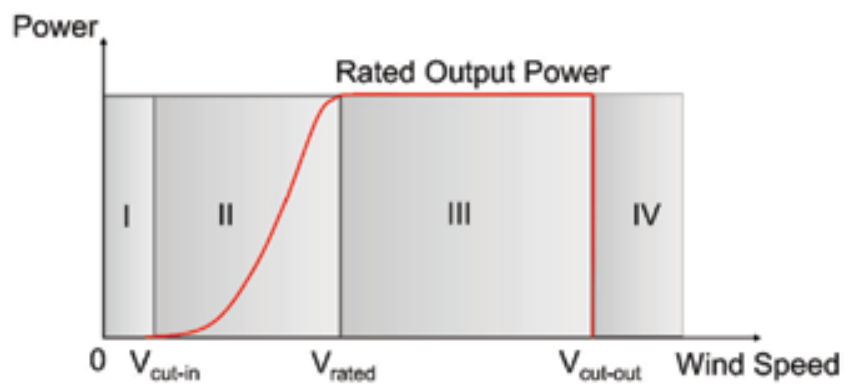

Figure 2.

A typical power curve of WTs with four operational stages I-IV.

speed and a cutout wind speed, and (4) zero power when the inflow wind speed is larger than the cutout wind speed (Figure 2).

\subsection{Exergy analysis}

In thermodynamics, the exergy of a system is defined as the maximum amount of useful work during a process that can bring the system into equilibrium with a reference environment [13]. Based on the second law of thermodynamics, exergy analysis is an alternative useful tool for analysis, evaluation, and design of many power and energy systems, e.g., renewable and traditional energy systems. The significant difference between energy and exergy analyses may be characterized as [6]:

1. In real irreversible process, exergy is always consumed; thus it is not subjected to a conservation law. In contrast, energy is neither created nor destroyed, but changing from one form to another, during a process. Thus, it is subjected to the conservation of energy law.

2. Although from a theoretical point of view exergy may be defined without a reference environment, it is often defined as a quantity relative to a specified reference environment and is equal to zero when it is in equilibrium with the reference environment.

The total exergy Ex of a flow with unit mass generally consists of four parts, which can be expressed as

$$
E x=E x_{k i}+E x_{p o}+E x_{p h}+E x_{c h}
$$

where $E x_{k i}, E x_{p o}, E x_{p h}$, and $E x_{c h}$ represent the kinetic, potential, physical, and chemical exergies, respectively. For thermodynamic analysis of WT systems, the potential exergy and chemical exergy are negligible in the total exergy. Thus, the total exergy for a WT can be reduced as

$$
E x=E x_{k i}+E x_{p h}
$$

where the kinetic exergy is defined herein as the maximum possible available kinetic energy that the air flow can produce from a wind speed to a complete stop and the physical exergy includes the enthalpy and entropy changes related to the turbine operation. The physical exergy can be calculated as $[6,7]$. 


$$
E x_{p h}=c_{p}\left(T_{2}-T_{1}\right)+T_{0}\left(c_{p} \ln \left(\frac{T_{2}}{T_{1}}\right)-R \ln \left(\frac{P_{2}}{P_{1}}\right)-\frac{c_{p}\left(T_{0}-T_{a v e}\right)}{T_{0}}\right)
$$

where the first term and the second term on the right side of Eq. (14) are the enthalpy and entropy contributions, respectively. $c_{p}$ is the specific heat of the flow; $T_{0}, T_{1}, T_{2}, T_{\text {ave }}$ are the reference temperature, inlet temperature, outlet temperate, and average temperature, respectively; $P_{1}$ and $P_{2}$ are the inlet pressure and outlet pressure, respectively (see Figure 1); and $R$ is a constant related to the gas and water vapor constants. Ideally, temperature and pressure at both inlet and outlet are needed to calculate the physical exergy. However, it is cumbersome to measure the temperatures and pressures at both inlet and outlet for the WT stream tube in real applications, not to mention the situation when evaluating the wind energy resource and/or WT efficiency performance before deploying WTs. In addition, the meteorological variable humidity is not considered in Eq. (14). To handle this difficulty, other studies have provided another formula to calculate the physical exergy for wind energy $[3,5,14,15]$ :

$$
\begin{aligned}
E x_{p h}= & \left(c_{p, a}+\omega c_{p, v}\right)\left(T-T_{0}\right) \\
& -T_{0}\left[\left(c_{p, a}+\omega c_{p, v}\right) \ln \left(\frac{T}{T_{0}}\right)-\left(R_{a}+\omega R_{v}\right) \ln \left(\frac{p}{P_{0}}\right)\right] \\
& +T_{0}\left[\left(R_{a}+\omega R_{v}\right) \ln \left(\frac{1+1.6078 \omega_{0}}{1+1.6078 \omega}\right)+1.6078 \omega R_{a} \ln \left(\frac{\omega}{\omega_{0}}\right)\right]
\end{aligned}
$$

where $c_{p, a}$ and $c_{p, v}$ are specific heat of air and water vapor, respectively; $\omega_{0}$ and $\omega$ are the humidity ratio of air at the reference state and at the current state, respectively; $R_{a}$ and $R_{v}$ are the gas constant and the water vapor constant, respectively; $T_{0}$ and $P_{0}$ are the reference temperature and atmospheric pressure, respectively; and $T$ and $p$ are measured temperature and pressure in this study.

\subsection{Energy and exergy efficiencies}

The efficiency for wind energy systems is explained by using energy efficiency $\eta$ and exergy efficiency $\psi$. The former is obtained as the ratio of useful energy produced by a WT to the total input wind energy, while the latter is defined as the useful exergy created by a WT to the total exergy of the air flow. These general definitions of energy and exergy efficiencies have been introduced in several literature (e.g., $[3,5-7,16])$. However, the specific definitions of useful energy/exergy for wind energy systems are often not very clearly explained in the literature. In order to avoid confusion, here we define that both the useful energy and useful exergy are equal to the rate of electricity output $E_{\text {out }}$ that a WT can produce under a wind speed (i.e., $E_{\text {out }}$ equals to actual output power $P_{\text {out }}$ ). Thus, the energy efficiency and exergy efficiency are calculated as, respectively,

$$
\begin{gathered}
\eta=\frac{E_{\text {out }}}{W_{\text {wind }}} \\
\psi=\frac{E_{\text {out }}}{E x}
\end{gathered}
$$

where $W_{\text {wind }}$ is the total input wind energy equal to the total kinetic energy given in Eq. (1) and Ex is the total exergy given in Eq. (13). By incorporating the 
meteorological variables and referring Eqs. (9)-(11), the energy efficiency can be expressed as

$$
\eta=\frac{2\left(R_{a}+\omega R_{v}\right)}{1+\omega} \frac{T P_{o u t}}{p A V_{1}^{3}}
$$

where $P_{\text {out }}$ is the output power defined by the power curve (see Figure 2). By Eqs. (13), (15), and (17), the exergy efficiency can be reorganized as

$$
\psi=\frac{P_{\text {out }}}{\left\{\begin{array}{l}
\frac{(1+\omega) p A V_{1}^{3}}{2\left(R_{a}+\omega R_{v}\right) T}+\left(c_{p, a}+\omega c_{p, v}\right)\left(T-T_{0}\right) \\
-T_{0}\left[\left(c_{p, a}+\omega c_{p, v}\right) \ln \left(\frac{T}{T_{0}}\right)-\left(R_{a}+\omega R_{v}\right) \ln \left(\frac{p}{P_{0}}\right)\right] \\
+T_{0}\left[\left(R_{a}+\omega R_{v}\right) \ln \left(\frac{1+1.6078 \omega_{0}}{1+1.6078 \omega}\right)+1.6078 \omega R_{a} \ln \left(\frac{\omega}{\omega_{0}}\right)\right]
\end{array}\right\}}
$$

Eqs. (18) and (19) derive the energy and exergy efficiencies given various meteorological variables, which can offer a straightforward evaluation of WT efficiency performance in a perspective of energy and exergy before deploying WTs. Hence, it will be beneficial in wind resource evaluation, wind farm site selection, and new WT design.

\section{Case study}

Using the presented thermodynamic analysis methods for wind energy systems, the wind energy potential is evaluated by investigating the energy and exergy efficiencies of a Goldwind 1.5 MW WT (model GW82/1500) [17], which is assumed to be deployed at Ithaca, New York, where 18-year reanalysis meteorological data are obtained from the Modern-Era Retrospective analysis for Research and Application, version 2 (MERRA-2), the latest atmospheric reanalysis of the modern satellite era produced by NASA's Global Modeling and Assimilation Office [18]. This section explains the site; the meteorological data including wind speed, pressure, temperature, and humidity; and the characteristics of the WT used for thermodynamic analysis.

\subsection{Site and data}

The wind energy potential is evaluated at Ithaca, which has moderately complex terrain in a landscape dominated by patches of forest, crop fields, hills, waterfalls, and lakes in the Upstate New York (at approximately $42.44^{\circ} \mathrm{N}, 76.50^{\circ}$ W, Figure 3). Experiencing a moderate continental climate, Ithaca has long, cold, and snowy winters and warm and humid summers with a dominance of westerly wind flows. The meteorological data are obtained from the MERRA-2 (a meteorological reanalysis data set created by NASA), which has a resolution of $0.5^{\circ}$ latitude $\times 0.625^{\circ}$ longitude [19]. Although it does not provide measured data in fields, the meteorological reanalysis is thought as a valuable tool to estimate the long-term variables, such as wind speed and temperature, for subsequent meteorological, climatological, energy, and environmental studies. By specifying the latitude and longitude of Ithaca, five types of meteorological data are retrieved from the 


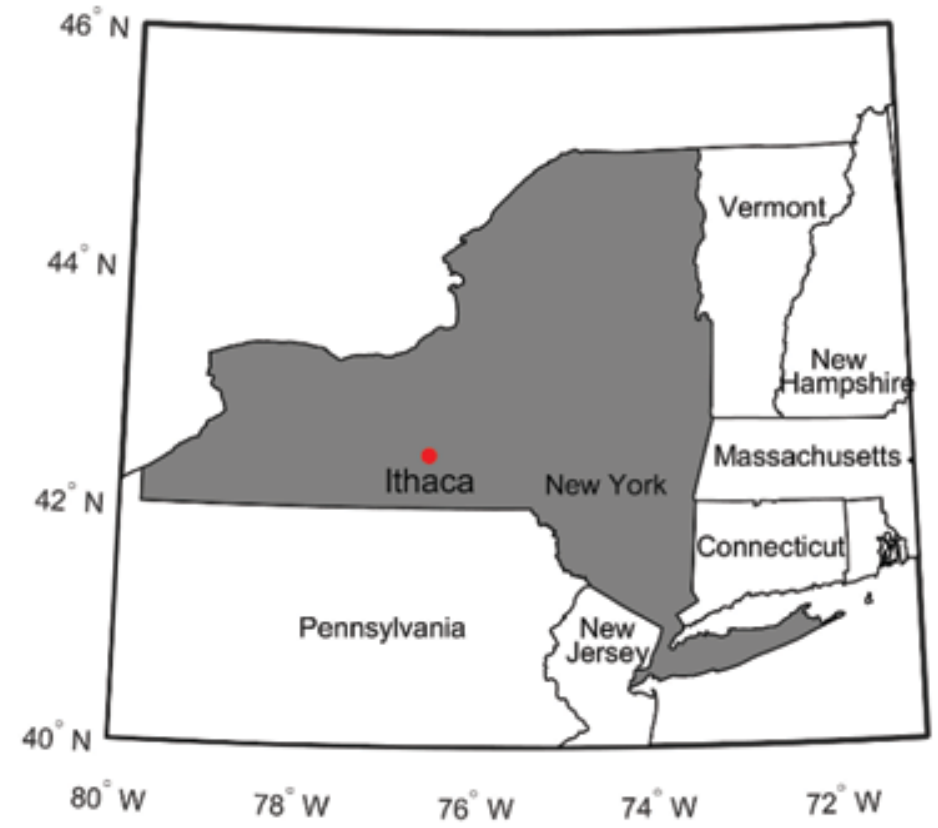

Figure 3.

Location of Ithaca, New York, where thermodynamic analysis of a 1.5 WM WT is investigated.

MERRA-2 including 10-m eastward wind U10M (in $\mathrm{ms}^{-1}$ ), 10-m northward wind V10M (in $\mathrm{ms}^{-1}$ ), surface pressure PS (in Pa), 10-m air temperature T10M (in K), 10-m specific humidity QV10M (in $\mathrm{kg} \mathrm{kg}^{-1}$ ), as well as their hourly time stamps from January 2000 to December 2017. The 10-m horizontal wind speed U is calculated as $U=\sqrt{U 10 M^{2}+V 10 M^{2}}$, and the humidity ratio $\omega$ is calculated from the specific humidity as $\omega=\mathrm{QV} 10 \mathrm{M} /(1-\mathrm{QV} 10 \mathrm{M})$. In total, there are 18 years of hourly meteorological data used for the thermodynamic analysis of the WT, which is assumed to be deployed in Ithaca, New York.

\subsection{Wind turbine}

The expected wind energy that can be harvested at a location is highly related to the WT characteristics, e.g., power curve and the available wind resources. Herein a Goldwind 1.5 MW permanent magnet direct-drive (PMDD) WT (GW85/1500) is assumed to be deployed at Ithaca area and used for evaluating the WT's energy and exergy efficiencies. Table 1 provides a summary of technical specifications of the WT. Since this study investigates the WT efficiency performance before real deployment, measured output power data are not available. It is assumed that the WT is performing perfectly according to its power curve, which consists of four operational stages (Figure 2). The WT starts to produce electricity at its cut-in wind speed of $3 \mathrm{~ms}^{-1}$, and the produced power is increased to the rated one of $1.5 \mathrm{MW}$ at the rated wind speed of $10.3 \mathrm{~ms}^{-1}$. In order to mitigate the fatigue and structural loadings under sustained high wind, WT control systems (e.g., the active blade pitch control) are operated to maintain the aerodynamic loads applied on blades and control the output power to be constant at the rated power. The WT is stopped, when wind speed is larger than the cut-out wind speed of $22 \mathrm{~ms}^{-1}$, to keep the whole turbine safe under extreme wind conditions. In this study, the power curve is represented by a six-order polynomial equation of wind speed during the cut-in and rated speeds, which is expressed as 


\begin{tabular}{lc}
\hline IEC wind class & IIIA \\
\hline Rated power $(\mathrm{kW})$ & 15,000 \\
\hline Cut-in wind speed $\left(\mathrm{ms}^{-1}\right)$ & 10.3 \\
\hline Rated wind speed $\left(\mathrm{ms}^{-1}\right)$ & 22 \\
\hline Cutout wind speed $\left(\mathrm{ms}^{-1}\right)$ & 5325 \\
\hline Swept area $\left(\mathrm{m}^{2}\right)$ & 3 \\
\hline Number of blades & 90 \\
\hline Hub height $(\mathrm{m})$ & Active blade pitch control \\
\hline Power control & PMDD synchronous generator \\
\hline Generator & 690 \\
\hline Rated voltage $(\mathrm{V})$ & 3 induction motors with hydraulic brakes \\
\hline Yaw system & Tubular steel tower \\
\hline Tower & Flat foundation \\
\hline Foundation & Microprocessor controlled with remote monitoring \\
\hline Converter &
\end{tabular}

Table 1.

Technical specifications of the Goldwind 1.5 MW PMDD WT [17].

$$
P_{\text {out }}=\left\{\begin{array}{l}
0, \quad V_{1}<3 \mathrm{~ms}^{-1} \text { or } V_{1}>22 \mathrm{~ms}^{-1} \\
0.0184 V_{1}^{6}-1.3507 V_{1}^{5}+30.8477 V_{1}^{4}-320.8737 V_{1}^{3}+1699.2172 V_{1}^{2} \\
-4366.5508 V_{1}+4287.3549 \mathrm{~kW}, \quad 3 \mathrm{~ms}^{-1} \leq V_{1} \leq 10.3 \mathrm{~ms}^{-1} \\
1500 \mathrm{~kW}, \quad 10.3 \mathrm{~ms}^{-1}<V_{1} \leq 22 \mathrm{~ms}^{-1}
\end{array}\right.
$$

\section{Results and discussion}

With the available meteorological data and the selected WT properties, assumptions are made for calculating the energy and exergy efficiencies: (1) the air pressure, temperature, and humidity are not significantly changed in the swept area of the WT. Thus, the surface pressure data, 10-m air temperature, and 10-m specific humidity obtained from the MERRA-2 data are directly used for the thermodynamic analyses. (2) Due to the wind shear effect in the atmospheric boundary layer, the normal wind profile model with a power law exponent of 0.2 is used to convert the $10-\mathrm{m}$ horizontal wind speed to the hub-height $(90 \mathrm{~m})$ wind speed according to the IEC standard [20]. It takes about 0.5 hour to convert six channels (five meteorological channels and one channel for time stamps) from the MERRA-2 netCDF4 data to Matlab data and then to calculate 18 years' hourly energy and exergy efficiencies using the developed Matlab scripts. Results and discussion are elaborated in three aspects: (1) WT efficiency variation in time domain, (2) meteorological variables impact on the efficiencies, and (3) uncertainty of meteorological variables represented by the best-fit distributions.

\subsection{Variation of energy and exergy efficiencies in time domain}

The energy and exergy efficiencies of the Goldwind WT are calculated by Eqs. (18) and (19), respectively, using the Ithaca meteorological data (wind speed, 
pressure, temperature, and humidity) retrieved from the MERRA-2 data set. As demonstrated in Figure 4, the variation of energy and exergy efficiencies is more closely following the variation of wind speed comparing with the other three meteorological variables, as wind power is proportional to the cubic of wind speed. Both efficiencies become 0 when the wind speed is less than the cut-in wind speed due to the WT being in idling status at the very low wind speed. As the WT is stopped when wind speed is larger than the cutout wind speed, the efficiencies are also equal to 0 . In addition, the energy efficiency present a higher magnitude than that of exergy efficiency, which is consistent with the theoretical derivations (Eqs. (18) and (19)) and previous findings (e.g., $[6,7])$. The difference between the two efficiencies is due to exergy destruction caused by irreversibility [7]. The concurrent low temperature and humidity ratio also demonstrate the cold and dry weather in winter of Ithaca.

Figure 5 shows the mean and standard deviation of energy and exergy efficiencies in different years and months. Annual means of energy and exergy efficiencies are smaller than the corresponding standard deviations, which indicates a significant variation of WT efficiency performance in 1 year as also demonstrated in Figure 4(e). Neither energy efficiency nor exergy efficiency exhibits clear trend from 2000 to 2017, even though relatively small and large means are observed in 2005 and 2014, respectively (Figure 5(a)). However, both mean and standard deviation of energy and exergy efficiencies present smaller values in summer than those in winter (Figure 5(b)). This seasonal change of efficiencies is likely related to the fact that high sustained wind speeds with strong variation more frequently occur in winter than in summer at the Ithaca area.

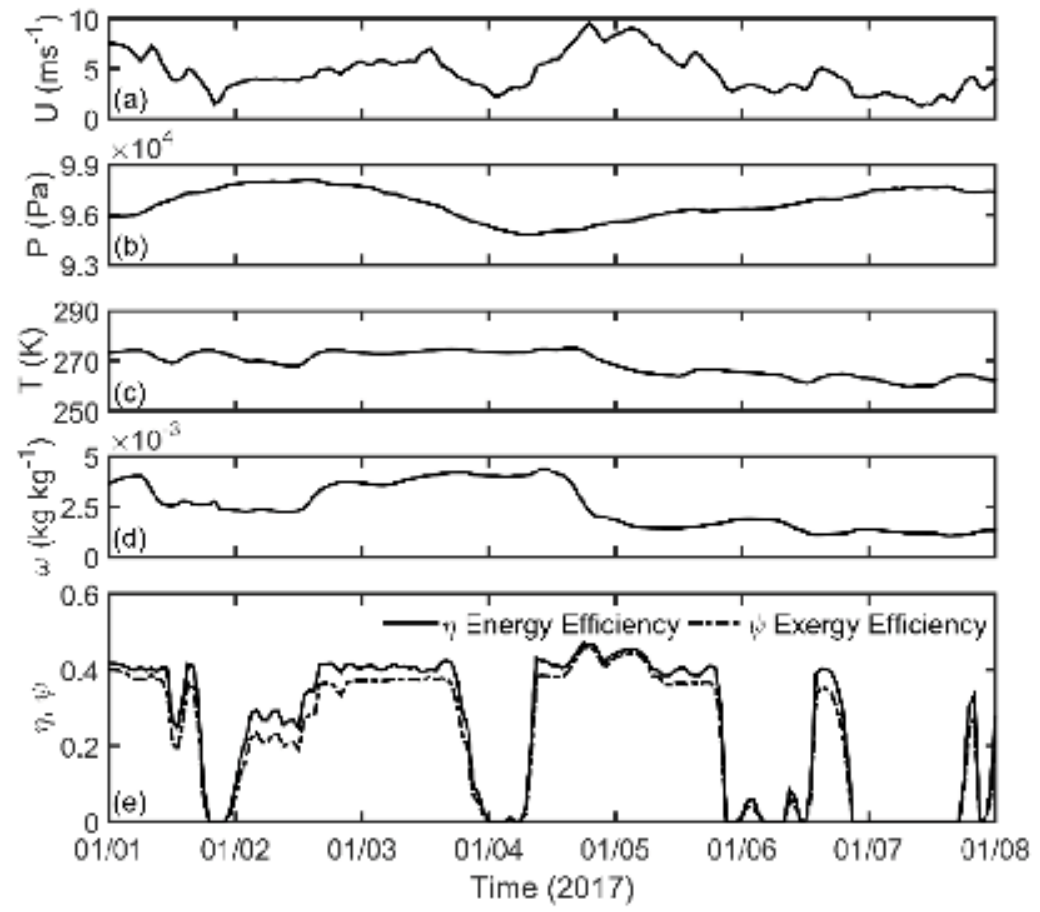

Figure 4.

Time series of hourly concurrent $(a)$ wind speed $U,(b)$ pressure $P,(c)$ temperature $T,(d)$ humidity ratio $\omega$, and $(e)$ energy efficiency $\eta$ and exergy efficiency $\psi$ during January 1-7, 2017. 

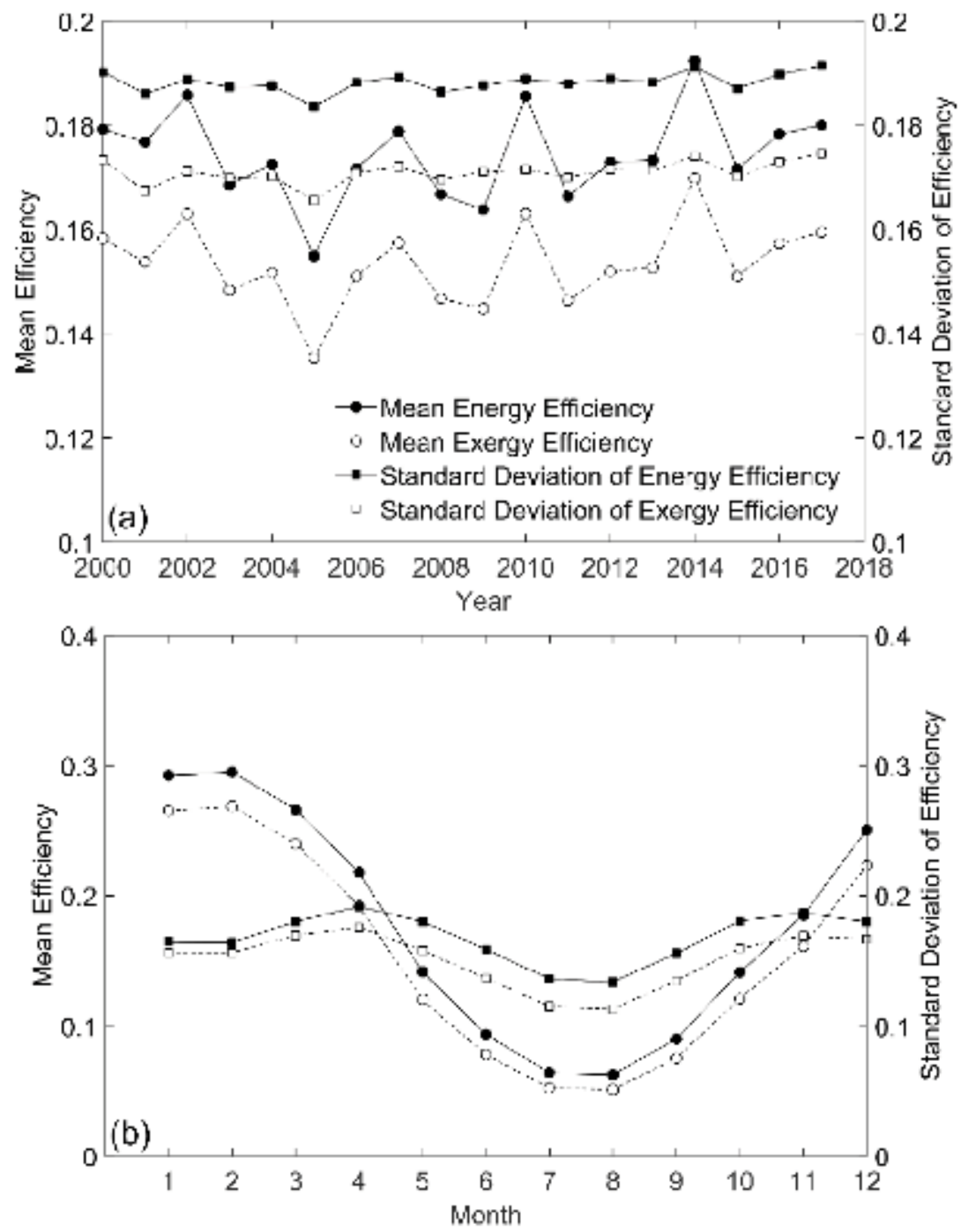

Figure 5.

Mean and standard deviation of energy and exergy efficiencies in different (a) years and (b) months.

\subsection{Impact of meteorological variables on energy and exergy efficiencies}

Relationships between the WT efficiencies and meteorological variables offer the trends of WT efficiency performance as meteorological variables change. Figure 6 shows the scatter diagrams of energy and exergy efficiencies versus the four meteorological variables (wind speed, pressure, temperature, and humidity ratio), as well as their relationships represented by different metrics. A bimodal relationship between the efficiencies and wind speed is observed due to the nonlinearity of the efficiency function with respect to wind speed (Figure 6(a)). The mean curves in Figure 6(a) show that the maximum means of energy and exergy efficiencies are $46.2 \%$ and $45.2 \%$, respectively, at the high peaks when the wind speed is equal to $\sim 9.2 \mathrm{~ms}^{-1}$, while the counterparts at the low peaks are $42.7 \%$ and $38.1 \%$ when the wind speed is equal to $\sim 5 \mathrm{~ms}^{-1}$. Despite the large variation, the efficiencies are linearly proportional to temperature and to the inverse of pressure (Figure 6(b and c)). Figure 6(d) shows that both the energy and exergy 

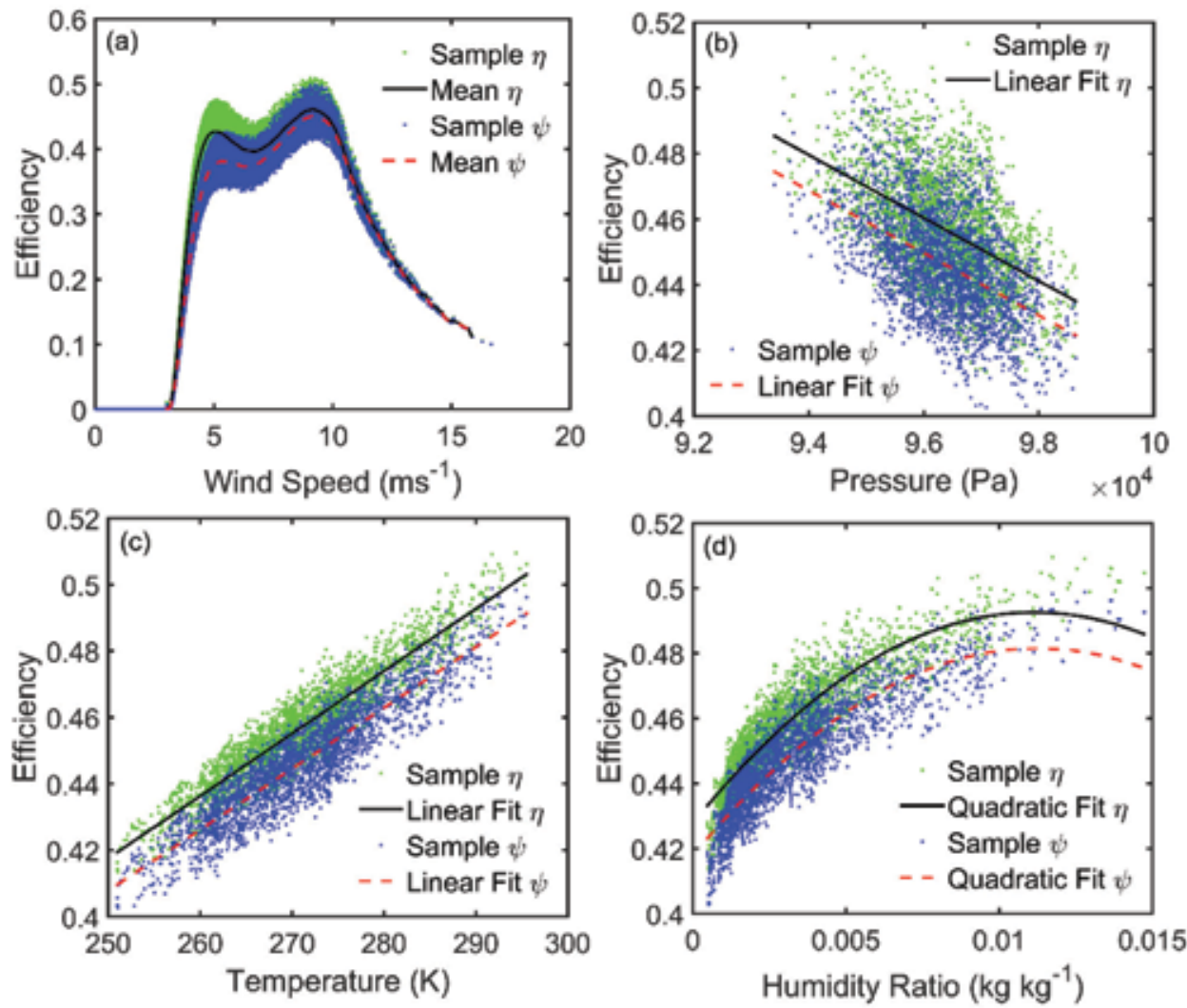

Figure 6.

Relationships between the WT efficiency (energy efficiency $\eta$ and exergy efficiency $\psi$ ) and meteorological variables including (a) wind speed, (b) pressure, (c) temperature, and (d) humidity ratio. All 18-year samples of hourly $\eta$ and $\psi$ versus wind speed are used in (a). For demonstration, samples in $(b),(c)$, and $(d)$ are conditionally sampled under a wind speed bin of $9 \mathrm{~ms}^{-1}$ (bin width $1 \mathrm{~ms}^{-1}$ ).

efficiencies are increased by $\sim 8 \%$ as the humidity ratio is increased from 0.001 to $0.015 \mathrm{~kg} \mathrm{~kg}^{-1}$, which indicates humidity plays an important role in affecting the WT efficiency performance.

\subsection{Uncertainties of meteorological variables and WT efficiencies}

Variation of meteorological variables could have significant impact on not only energy and exergy efficiencies as explained in Section 4.2 but also many other aspects, e.g., fatigue and structural reliability. Although Weibull distribution is often used to represent the uncertainty of mean wind speed in long term [21, 22], few previous studies have sought to address which parent distribution best represents other meteorological variables, e.g., pressure, temperature, and humidity for WT analyses. This is an important omission since these meteorological variables could have critical roles, but maybe indirectly, to WT performance. For example, high air humidity, low wind speed, and temperature above $\sim 10^{\circ} \mathrm{C}$ are preferred by insects that will increasingly foul the leading edges of WT blades and contaminate the blade surface eventually decreasing the aerodynamic performance [23]. Since both the wind speed and pressure considered herein are zero bounded, four positive-valued distribution types (Weibull, lognormal, gamma, and log-logistic; see Figure 7(a and $\mathbf{b})$ ) are fitted to wind speed and pressure using maximum likelihood estimation (MLE). Due to the clear two-peak histograms observed for 

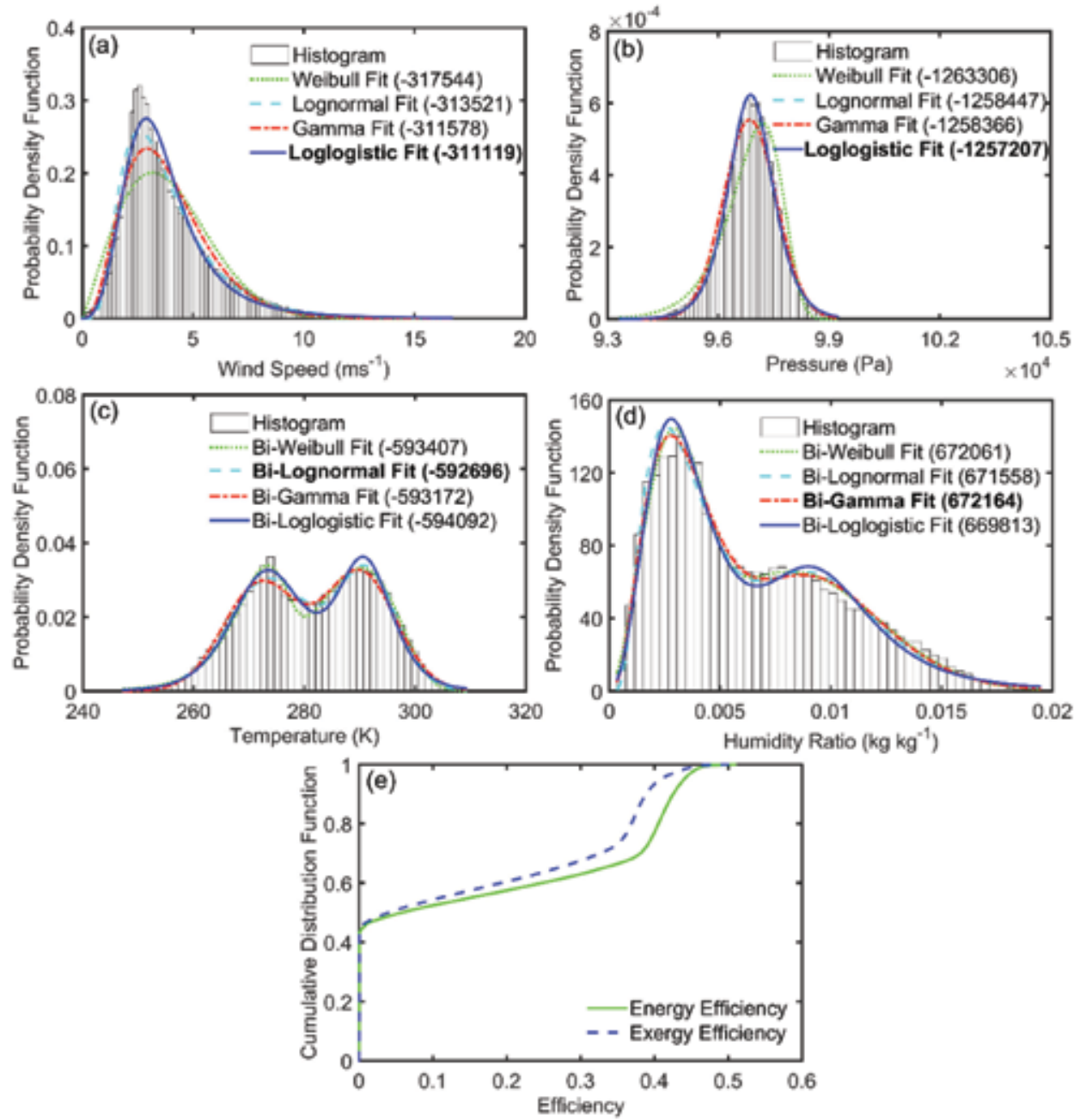

Figure 7.

Histograms and distribution fits for (a) wind speed, (b) pressure, (c) temperature, and (d) humidity ratio; and (e) empirical cumulative distribution function of energy and exergy efficiencies. In the legends, the loglikelihood values are in parentheses. The bolded distribution with the largest log-likelihood value is selected as the best-fit distribution and is summarized in Table 2. Recall all 18-year samples of hourly meteorological data, and the calculated WT energy and exergy efficiencies are used in Figure 7.

temperature and humidity ratio, four positive-valued bimodal distributions (biWeibull, bi-lognormal, bi-gamma, and bi-log-logistic) are fitted to temperature and humidity ratio (Figure $7(\mathbf{c}$ and $\mathbf{d})$ ). The probability density function (PDF) of a bimodal distribution consists of two PDFs with the same distributional type, which is expressed as

$$
f\left(x \mid w, a_{1}, b_{1}, a_{2}, b_{2}\right)=w f\left(x \mid a_{1}, b_{1}\right)+(1-w) f\left(x \mid a_{2}, b_{2}\right)
$$

where $x$ represents a meteorological variable; $\left(a_{1}, \mathrm{~b}_{1}\right)$ and $\left(a_{2}, \mathrm{~b}_{2}\right)$ are the parameters of the first and the second constituent PDFs, respectively; and $w$ is the weight for the constituent distributions $f\left(x \mid a_{1}, b_{1}\right)$ in the bimodal distribution form. The candidate distribution with the largest log-likelihood value is selected as the best-fit distribution [24].

Figure 7 and Table 2 summarize the distributional fits for the four meteorological variables. It is found that the log-logistic distribution is best fit for wind speed 


\begin{tabular}{|c|c|c|}
\hline $\begin{array}{l}\text { Meteorological } \\
\text { variables }\end{array}$ & $\begin{array}{l}\text { Best-fit } \\
\text { distribution } \\
\text { type }\end{array}$ & Probability density function \\
\hline $\begin{array}{l}\text { Wind speed } \\
\left(\mathrm{ms}^{-1}\right)\end{array}$ & $\begin{array}{l}\text { Log-logistic } \\
\text { distribution }\end{array}$ & $\begin{aligned} f(x \mid a, b) & =\frac{1}{b} \frac{1}{x} \frac{\exp (z)}{[1+\exp (z)]^{2}} \\
\text { where } z & =\frac{\ln (x)-a}{b}, a=1.2307, b=0.2887\end{aligned}$ \\
\hline Pressure $(\mathrm{Pa})$ & $\begin{array}{l}\text { Log-logistic } \\
\text { distribution }\end{array}$ & $\begin{array}{l}f(x \mid a, b)=\frac{1}{b} \frac{1}{x} \frac{\exp (z)}{[1+\exp (z)]^{2}} \\
\text { where } z=\frac{\ln (x)-a}{b}, a=11.4812, b=0.0041\end{array}$ \\
\hline $\begin{array}{l}\text { Temperature } \\
\text { (K) }\end{array}$ & $\begin{array}{l}\text { Bi-lognormal } \\
\text { distribution }\end{array}$ & $\begin{aligned} & f\left(x \mid w, a_{1}, b_{1}, a_{2}, b_{2}\right)= w \frac{1}{x b_{1} \sqrt{2 \pi}} \exp \left[\frac{-\left(\ln x-a_{1}\right)^{2}}{2 b_{1}^{2}}\right] \\
&+(1-w) \frac{1}{x b_{2} \sqrt{2 \pi}} \exp \left[\frac{-\left(\ln x-a_{2}\right)^{2}}{2 b_{2}^{2}}\right] \\
& w=0.5824, a_{1}=5.6125, b_{1}=0.0280, a_{2}=5.6752, b_{2}=0.0182\end{aligned}$ \\
\hline $\begin{array}{l}\text { Humidity ratio } \\
\left(\mathrm{kg} \mathrm{kg}^{-1}\right)\end{array}$ & $\begin{array}{l}\text { Bi-gamma } \\
\text { distribution }\end{array}$ & $\begin{aligned} f\left(x \mid w, a_{1}, b_{1}, a_{2}, b_{2}\right) & =w \frac{1}{b_{1}^{a_{1}} \Gamma\left(a_{1}\right)} x^{a_{1}-1} \exp \left(-\frac{x}{b_{1}}\right) \\
& +(1-w) \frac{1}{b_{2}^{a_{2}} \Gamma\left(a_{2}\right)} x^{a_{2}-1} \exp \left(-\frac{x}{b_{2}}\right), \\
w=0.5970, a_{1}= & 3.8190, b_{1}=0.0010, a_{2}=12.6287, b_{2}=0.0008\end{aligned}$ \\
\hline
\end{tabular}

Table 2.

The best-fit distribution form and distribution parameters for the four meteorological variables.

and pressure (Figure 7( $\mathbf{a}$ and $\mathbf{b})$ ), despite the commonly used Weibull distribution for mean wind speed. The bi-lognormal and bi-gamma distributions are best fit for temperature and humidity ratio, respectively. The existence of bimodal shape of the distributions of temperature is likely related to the very distinguished high and low temperature corresponding to the summer and winter seasons, respectively, in Ithaca. The same reason explains the bimodal shape for humidity. The obtained specific distributions for the meteorological parameters, provided in Table 2, are readily applicable for WT performance analyses, i.e., fatigue, structure, aerodynamics, and thermodynamics, in moderately complex terrain of the northeastern United States. Figure 7(e) presents the empirical cumulative distribution function (CDF) of energy and exergy efficiencies calculated herein. Due to the large amount of 0 energy and exergy efficiencies when wind speed is below the cut-in wind speed, the CDF curves show that there is a probability of $\sim 43 \%$ that the efficiencies are equal to 0 . The largest discrepancy between CDF of energy and exergy efficiencies occurs at efficiencies equal to 0.4 . The presented CDF could be used to evaluate the reliability of wind power performance considering realistic meteorological uncertainty.

\section{Conclusions}

This chapter presents methods and results for thermodynamic analysis of wind energy systems considering four types of meteorological variables, i.e., wind speed, pressure, temperature, and humidity. An improved understanding of WT efficiencies is critically important and necessary before launching any wind projects. The evaluation of WT efficiencies considering thermodynamics, conducted here for an 
1.5 MW WT (Goldwind GW82/1500) potentially deployed at Ithaca, New York, is beneficial to WT design, siting, and operation in moderately complex terrain in the northeastern United States. The key concluding remarks are the following:

- The chapter offers the fundamental derivations of energy and exergy efficiencies of WTs considering wind speed, pressure, temperature, and humidity, which lay a foundation for the thermodynamic analysis of wind energy systems.

- The WT energy efficiency presents higher magnitude than exergy efficiency based on the theoretical derivation and the calculated time series of efficiencies. There is no clear trend of annual variations of mean and standard deviation of both energy and exergy efficiencies. However, a clear seasonal change is found that energy and exergy efficiencies studied herein have smaller values in summer than those in winter.

- Although wind speed has a dominating influence, other meteorological variables (i.e., pressure, temperature, and humidity) do have a considerable impact on the WT efficiency performance. The WT efficiencies are linearly associated with pressure and temperature, while it has highly nonlinear relationships with wind speed and humidity ratio.

- Log-logistic distributions are most appropriate for the wind speed and pressure data retrieved from the MERRA-2 data set at Ithaca, New York. A bi-lognormal distribution and a bi-gamma distribution are most appropriate for the temperature and humidity ratio, respectively. The obtained PDFs of meteorological variables and CDFs of energy and exergy efficiencies could be beneficial for evaluating the reliability of wind power performance considering realistic meteorological uncertainty in the northeastern United States.

Naturally the specific findings are based on reanalysis meteorological data and the assumed WT deployment; the methodologies of thermodynamic analysis presented here are applicable for real measured meteorological data and recorded WT performance somewhere else if available. In addition, although the thermodynamic analysis of wind energy systems in this chapter focuses on energy and exergy efficiencies, other variables, e.g., dynamic response, fatigue damage, structural deformation, etc., of the PMDD WT are also potentially affected by the meteorological variables, which could be investigated in the future.

\section{Acknowledgements}

Support from the National Natural Science Foundation of China (grant numbers 51475417, U1608256, and 51521064) is gratefully acknowledged. Weifei Hu would like to appreciate Dr. Qinjian Jin and Dr. Frederick Letson at Department of Earth and Atmospheric Sciences, Cornell University, for the introduction and discussion of the MERRA-2 data.

\section{Conflict of interest}

The authors certify that this work has no conflict of interest with any organization or entity in the subject matter or materials discussed in this chapter. 


\section{Nomenclature}

\begin{tabular}{|c|c|}
\hline$A$ & rotor swept area \\
\hline$C_{P}$ & power coefficient \\
\hline$c_{p}, c_{p, a}, c_{p, v}$ & $\begin{array}{l}\text { specific heat of flow, specific heat of air, specific heat of water } \\
\text { vapor }\end{array}$ \\
\hline$E_{k}$ & kinetic energy \\
\hline$E x$ & exergy \\
\hline$E x_{k i}$ & kinetic exergy \\
\hline$E x_{p o}$ & potential exergy \\
\hline$E x_{p h}$ & physical exergy \\
\hline$E x_{c h}$ & chemical exergy \\
\hline$f$ & PDF of meteorological variables \\
\hline$m$ & mass \\
\hline$P$ & wind power/wind pressure \\
\hline$P_{0}, P_{1}, P_{2}, P_{\text {ave }}, p$ & $\begin{array}{l}\text { reference pressure, inlet pressure, outlet pressure, average } \\
\text { pressure, and wind pressure in humid air, respectively }\end{array}$ \\
\hline PS & surface pressure retrieved from the MERRA-2 data set \\
\hline QV10M & 10-m specific humidity retrieved from the MERRA-2 data set \\
\hline$R_{a}, R_{v}$ & gas constant and water vapor constant, respectively \\
\hline$T_{0}, T_{1}, T_{2}, T_{\text {ave }}$ & $\begin{array}{l}\text { reference temperature, inlet temperature, outlet temperature, } \\
\text { and average temperature, respectively }\end{array}$ \\
\hline T10M & 10-m air temperature retrieved from the MERRA-2 data set \\
\hline$U$ & 10-m horizontal wind speed \\
\hline U10M & $\begin{array}{l}\text { 10-m eastward wind speed retrieved from the MERRA- } 2 \text { data } \\
\text { set }\end{array}$ \\
\hline V10M & $\begin{array}{l}\text { 10-m northward wind speed retrieved from the MERRA-2 } \\
\text { data set }\end{array}$ \\
\hline$V$ & wind speed \\
\hline$W_{\text {wind }}$ & total input wind energy \\
\hline$\eta$ & energy efficiency \\
\hline$\psi$ & exergy efficiency \\
\hline$\rho$ & air density $\left[\mathrm{kgm}^{-3}\right]$ \\
\hline$\omega_{0} / \omega$ & humidity ratio of air at the reference state/at the current state \\
\hline MERRA-2 & $\begin{array}{l}\text { Modern-Era Retrospective Analysis for Research and Applica- } \\
\text { tions, Version } 2\end{array}$ \\
\hline NASA & National Aeronautics and Space Administration \\
\hline PMDD & permanent magnet direct-drive \\
\hline WTs/WT & ind turbines/wind turbine \\
\hline
\end{tabular}




\section{Author details}

Weifei $\mathrm{Hu}^{1}$, Zhenyu Liu ${ }^{2 *}$ and Jianrong $\operatorname{Tan}^{2}$

1 School of Mechanical Engineering, Zhejiang University, Hangzhou, P.R. China

2 State Key Laboratory of CAD \& CG, Zhejiang University, Hangzhou, P.R. China

*Address all correspondence to: liuzy@zju.edu.cn

\section{IntechOpen}

(C) 2019 The Author(s). Licensee IntechOpen. This chapter is distributed under the terms of the Creative Commons Attribution License (http://creativecommons.org/licenses/ by/3.0), which permits unrestricted use, distribution, and reproduction in any medium, provided the original work is properly cited. (cc) BY 


\section{References}

[1] Global Wind Energy Council. Global Wind Statistics. 2016. Available from: http://www.gwec.net/wp-content/ uploads/vip/GWEC_PRstats2016_EN_ WEB.pdf [Accessed: June 1, 2018]

[2] U.S. Department of Energy. 20\% Wind Energy by 2030-Increasing Wind Energy's Contribution to U.S. Electricity Supply. U.S. Department of Energy, Office of Scientific and Technical Information: Oak Ridge, TN; 2008

[3] Baskut O, Ozgener O, Ozgener L. Effects of meteorological variables on exergetic efficiency of wind turbine power plants. Renewable and Sustainable Energy Reviews. 2010;14: 3237-3241

[4] Krakow KI. Exergy analysis: Deadstate definition. ASHRAE Transactions. 1991;97:328-336

[5] Ozgener O, Ozgener L. Exergy and reliability analysis of wind turbine systems: A case study. Renewable and Sustainable Energy Reviews. 2007;11: 1811-1826

[6] Şahin AD, Dincer I, Rosen MA. Thermodynamic analysis of wind energy. International Journal of Energy Research. 2006;30:553-566

[7] Redha AM, Dincer I, Gadalla M. Thermodynamic performance assessment of wind energy systems: An application. Energy. 2011;36:4002-4010

[8] Lanchester FW. A contribution to the theory of propulsion and the screw propeller. Journal of the American Society for Naval Engineers. 1915;27: 509-510

[9] Betz A. Schraubenpropeller mit geringstem Energieverlust. Gottinger Nachrichten. 1919:193-213
[10] Burton T, Jenkins N, Sharpe D, Bossanyi E. Aerodynamics of horizontal axis wind turbine. In: Burton T, Jenkins $\mathrm{N}$, Sharpe D, Bossanyi E, editors. Wind Energy Handbook. 2nd ed. John Wiley \& Sons; 2011. pp. 39-136

[11] Moriarty PJ, Hansen AC. AeroDyn Theory Manual: National Renewable Energy Laboratory Golden. Colorado: USA; 2005. pp. 2-10

[12] Hansen MO. Aerodynamics of Wind Turbines. London: Routledge; 2015. pp. 63-77

[13] Rant Z. Exergie, ein neues wort fur 'technische arbeitsfaehigkeit' (exergy, a new word for technical availability). Forschung auf dem Gebiet des Ingenieurwesens A. 1956;22:36-37

[14] Wepfer W. Proper evaluation of available energy for HVAC. ASHRAE Transactions. 1979;85:214-230

[15] Dincer I, Sahin AZ. A new model for thermodynamic analysis of a drying process. International Journal of Heat and Mass Transfer. 2004;47:645-652

[16] Pope K, Dincer I, Naterer GF. Energy and exergy efficiency comparison of horizontal and vertical axis wind turbines. Renewable Energy. 2010;35:2102-2113

[17] Goldwind. 1.5 MW Permanent Magnet Direct-Drive (PMDD) Wind Turbine. Available from: https://www. goldwindamericas.com/15-mw-pmdd [Accessed: August 15, 2018]

[18] Gelaro R, McCarty W, Suárez MJ, Todling R, Molod A, Takacs L, et al. The modern-era retrospective analysis for research and applications, version 2 (MERRA-2). Journal of Climate. 2017; 30:5419-5454 
[19] NASA. MERRA-2: File specification. Greenbelt, Maryland: Global Modeling and Assimilation Office, Earth Sciences Division, NASA Goddard Space Flight Center; 2016

[20] International Electrotechnical Commission. Wind turbines-part I: Design requirements. IEC standard 61400-1. 3rd ed; 2005

[21] Hu W, Choi K, Zhupanska O, Buchholz JH. Integrating variable wind load, aerodynamic, and structural analyses towards accurate fatigue life prediction in composite wind turbine blades. Structural and Multidisciplinary Optimization. 2016;53:375-394

[22] Hu W, Choi K, Cho H. Reliabilitybased design optimization of wind turbine blades for fatigue life under dynamic wind load uncertainty. Structural and Multidisciplinary Optimization. 2016;54:953-970

[23] Dalili N, Edrisy A, Carriveau R. A review of surface engineering issues critical to wind turbine performance. Renewable and Sustainable Energy Reviews. 2009;13:428-438

[24] Hogg RV, Craig AT, McKean JW. Introduction to Mathematical Statistics. 6th ed. Upper Saddle River, New Jersey: Pearson Prentice Hall; 2005. pp. 311-317 



\title{
Advanced Monitoring of Wind Turbine
}

\author{
Steve Alan Talla Ouambo, Alexandre Teplaira Boum \\ and Adolphe Moukengue Imano
}

\begin{abstract}
This chapter presents a general framework for the doubly fed induction generator (DFIG). We apply and analyze the behavior of three estimation techniques, which are the unscented Kalman filter (UKF), the high gain observer (HGO) and the moving horizon estimation (MHE). These estimations are used for parameters estimation of the doubly fed induction generator (DFIG) driven by wind turbine. A comparison of those techniques has been made under different aspects notably, computation time and estimation accuracy in two modes of operation of the DFIG, the healthy mode and the faulty mode. The performance of the MHE has been clearly superior to other estimators during our experiments. These estimation tools can be used for monitoring purposes.
\end{abstract}

Keywords: doubly-fed induction generator, high gain observer, unscented Kalman filter, moving horizon estimation, parameters estimation, monitoring

\section{Introduction}

Nowadays most of generated electricity comes from nonrenewable sources of fuel. These products transfer to the atmosphere important quantities of $\mathrm{CO}_{2}$, and inescapably leading to the warming up of the atmosphere [1]. The production of the wind energy spreads through the world, and significantly, it imposed itself during the past decade [2]. Doubly-fed induction generators (DFIGs) are actually the most used wind power generators in many countries [3].

Therefore, many contributions have been made to the inverters and converters usually in DFIG used in the power electronics domain [4]. A doubly fed induction generator model for transient stability analysis has been proposed in [5], in which authors focused their study on the control loops of instantaneous response. In [6], authors have been proposed some robust observers to estimate states and actuator faults for different class of linear and nonlinear systems at the same instant. Though systems are becoming more and more complex, DFIG can be subject by many types of faults [7], diagnosis and faults estimation issues have become primordial to ensure a good supervision of systems and guarantee the safety of materials and operators (humans) [8].

A survey based on current sensor fault detection and isolation and control reconfiguration current for doubly fed induction generator has been proposed by [9]. Studies led by [10], have contributed to an adaptive parameter estimation algorithm used for estimating the rotor resistance of the DFIG, however, the others 
parameters were assumed to be constant. To improve the extended Kalman filter (EKF), a new nonlinear filtering algorithm named the unscented Kalman filter (UKF) has been developed in [11]. Widely used in some fields, UKF has been found in several studies such as training of neural networks [12], multi-sensor fusion for instance.

This chapter investigates the usage of the unscented Kalman filter UKF, high gain observer (HGO) and the moving horizon estimator (MHE) to estimate the dynamic states and electrical parameters of the wind turbine system. These estimates can be used to enhance the performance of doubly fed induction generator in power systems, for rotor and stator resistances faults in the circumstances where internal states will be involved in a control design [3] and the acquisition of internal states, which are relatively difficult to get can realized from the dynamic state estimation and for monitoring purposes. The chapter is organized as follows: in Section 2, the mathematical model for DFIG is presented, followed by the description of estimation algorithms in Section 3. The results of the parameter estimation tests are presented in Section 4. Finally Section 5 gives the conclusions.

\section{Mathematical model for DFIG}

In this section, we deal with the mathematical modeling of the DFIG-based wind energy system, we will only describe the wind turbine (also called drive train), and the asynchronous generator (also called induction generator) because this chapter focuses on estimating of the parameters and dynamic states of the DFIG Figure 1. Two frames of reference are used in this model: stator voltage $(\mathrm{d}-\mathrm{q})$ reference frame and mutual flux (d-q) reference frame. In Tables 1 and 2, all parameters and constants are given.

\subsection{Modeling of the wind turbine}

From the wind, the power extracted can give the mechanical torque. The energy from the wind is extracted from the wind turbine and converted into mechanical power [14]. The wind turbine model is based on the output power characteristics, as Eqs. (1) and (2), [15].

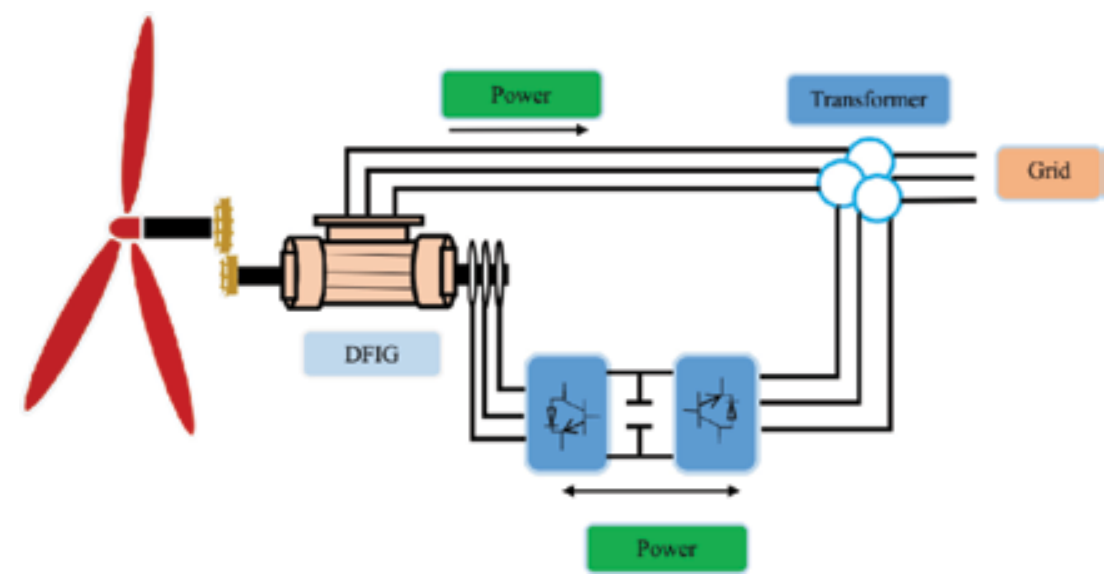

Figure 1.

Configuration of DFIG-based wind turbine system [13]. 


\begin{tabular}{lc}
\hline Parameters & Values \\
\hline Rated active power $(\mathrm{Ps}) /(\mathrm{MW})$ & 1.5 \\
\hline Rated voltage $($ line to line $)(\mathrm{Vs}) /(\mathrm{V})$ & 575 \\
\hline Rated DC-link voltage $(\mathrm{Vdc}) /(\mathrm{V})$ & 1200 \\
\hline Number of poles & 4 \\
\hline Frequency $(\mathrm{f}) /(\mathrm{Hz})$ & 60 \\
\hline Stator resistance $(\mathrm{Rs}) /(\mathrm{pu})$ & 0.00707 \\
\hline Rotor resistance $(\mathrm{Rr}) /(\mathrm{pu})$ & 0.005 \\
\hline Stator leakage inductance $(\mathrm{Ls}) /(\mathrm{pu})$ & 0.171 \\
\hline Rotor leakage inductance $(\mathrm{Lr}) /(\mathrm{pu})$ & 0.156 \\
\hline Magnetizing inductance $(\mathrm{Lm}) /(\mathrm{pu})$ & 2.9 \\
\hline DC-link capacitance $(\mathrm{C}) /(\mathrm{F})$ & 0.04 \\
\hline
\end{tabular}

Table 1.

Parameters of the DFIG.

\begin{tabular}{lc}
\hline Parameters & Values \\
\hline Rated wind speed $\left(\mathrm{v}_{\mathrm{w}}\right) /\left(\mathrm{m} \mathrm{s}^{-1}\right)$ & 12 \\
\hline Number of blade & 3 \\
\hline Radius of blade $(\mathrm{R}) / \mathrm{m}$ & 35.25 \\
\hline Gearbox gain $(\mathrm{G})$ & 91 \\
\hline Moment of inertia $\left(\mathrm{J}_{\mathrm{eq}}\right) /\left(\mathrm{kg} \mathrm{m}^{2}\right)$ & 1000 \\
\hline Viscosity factor $\left(\mathrm{f}_{\mathrm{eq}}\right) /\left(\mathrm{N} \mathrm{m} \mathrm{s} \mathrm{rad}^{-1}\right)$ & 0.0024 \\
\hline
\end{tabular}

Table 2.

Parameters of the wind turbine.

$$
\begin{gathered}
P_{m}=C_{p}(\lambda, \beta) \frac{1}{2} \rho A \nu_{w}^{3}=C_{p}(\lambda, \beta) E_{w} \\
\lambda_{T S}=\frac{R \omega_{t}}{\nu_{w}}
\end{gathered}
$$

where the aerodynamic extracted power is $P_{m}$, which depends on $C_{P}$, the efficiency coefficient,the air density $\rho$, the turbine swept area $\mathrm{A}$, and the wind speed $\nu_{w}$. The kinetic energy contained in the wind at a particular wind speed is given by $E_{w}$. The blade radius and angular frequency of rotational turbine are $R$ and $w_{t}$, respectively. $C_{P}(\lambda ; \beta)$ the efficiency coefficient depends on tip speed ratio $\lambda_{T S}$ and blade pitch angle $\beta$, determines the amount of wind kinetic energy that can be captured by the wind turbine system $[13] . C_{P}(\lambda ; \beta)$ can be described as:

$$
C_{p}(\lambda, \beta)=0.5\left(\frac{116}{\lambda_{i}}-0.4 \beta-5\right) e^{-21 / \lambda_{i}}
$$

where

$$
\frac{1}{\lambda_{i}}=\frac{1}{\lambda_{T S}+0.08 \beta}-\frac{0.035}{\beta^{3}+1}
$$




\subsection{Modeling of the asynchronous generator}

For the induction generator, the Park model is the model that is commonly used [16]. After applying the synchronously rotating reference frame transformation to the stator and rotor fluxes equations of the generator, the following differential equations describe the dynamics of the rotor and stator fluxes [17]:

$$
\left\{\begin{array}{l}
\dot{\Phi}_{d r}=w_{b}\left(v_{d r}+\left(w_{s}-w_{r}\right) \Phi_{q r}-R_{r} i_{d r}\right) \\
\dot{\Phi}_{q r}=w_{b}\left(v_{q r}-\left(w_{s}-w_{r}\right) \Phi_{d r}-R_{r} i_{q r}\right) \\
\dot{\Phi}_{d s}=w_{b}\left(v_{d s}+w_{s} \Phi_{q s}-R_{s} i_{d s}\right) \\
\dot{\Phi}_{q s}=w_{b}\left(v_{q s}-w_{s} \Phi_{d s}-R_{s} i_{q s}\right)
\end{array}\right.
$$

where $w_{s}=1$ is the synchronous angular speed in the synchronous frame and $w_{b}=2 \pi \mathrm{f} \mathrm{rad} / \mathrm{s}$ is the base angular speed, with $f=60 \mathrm{~Hz}$. With additional variables stator-rotor mutual flux $\Phi_{d m}$ and $\Phi_{q m}$, rotor current $i_{d r}$ and $i_{q r}$ and stator current ids and $i_{q s}$ can be expressed as:

$$
\begin{aligned}
& \left\{\begin{array}{l}
i_{d r}=\frac{\Phi_{d r}-\Phi_{d m}}{L_{l r}} \\
i_{q r}=\frac{\Phi_{q r}-\Phi_{q m}}{L_{l r}}
\end{array}\right. \\
& \left\{\begin{array}{l}
i_{d s}=\frac{\Phi_{d s}-\Phi_{d m}}{L_{l s}} \\
i_{q s}=\frac{\Phi_{q s}-\Phi_{q m}}{L_{l s}}
\end{array}\right.
\end{aligned}
$$

where

$$
\begin{aligned}
& \Phi_{d m}=L_{a d}\left(\frac{\Phi_{d r}}{L_{l r}}+\frac{\Phi_{d s}}{L_{l s}}\right) \\
& \Phi_{q m}=L_{a q}\left(\frac{\Phi_{q r}}{L_{l r}}+\frac{\Phi_{q s}}{L_{l s}}\right)
\end{aligned}
$$

are the stator-rotor mutual flux.

Where constants $L_{a d}$ and $L_{a q}$ are the $(d-q)$ mutual flux factors, expressed as:

$$
L_{a d}=L_{a q}=\frac{1}{\frac{1}{L_{m}}+\frac{1}{L_{l s}}+\frac{1}{L_{l r}}}
$$

The relationship between mechanical torque $T_{m}$, electrical torque $T_{e}$ and rotor speed $w_{r}$ can be shown by the following differential equation,

$$
\dot{w}_{r}=\frac{1}{2 H}\left(T_{m}-T_{e}-F w_{r}\right)
$$

where constant $F$ is the friction factor and $H$ is the generator inertia, and $T_{e}$, the electrical torque which can be expressed as:

$$
T_{e}=\Phi_{d s} i_{q s}-\Phi_{q s} i_{d s}
$$

These equations are derived in [4] and all parameters are defined in per unit based on the generator ratings and synchronous speed. 


\section{Estimation algorithms}

\subsection{High gain observer}

This observer class is applied for nonlinear system classes of the form Eq. (12). Its applications are so large $[18,19]$. We briefly present the developed survey in [20] that points up the synthesis of observers adapted to the observable nonlinear systems. Consider the following nonlinear system:

$$
\left\{\begin{array}{l}
\dot{x}=f(x)+g(x) u \\
y=h(x)
\end{array}\right.
$$

where $x \in R^{n}, u \in R^{m}, y \in R^{s}$.

First, the system Eq. (12) must be uniformly locally observable, and then it will be possible to make the variable change $z=\Gamma(x)$ that will transform the system Eq. (12) in the following form:

$$
\left\{\begin{array}{l}
\dot{z}=A z+\varphi(u, z) \\
y=C z
\end{array}\right.
$$

The observer must satisfy the following theorem [20]:

i. The function $\varphi$ is globally Lipschitz uniformly to $u$.

Let $K=\left[\begin{array}{lll}K_{1} & & \\ & \ddots & \\ & & K_{p}\end{array}\right]$ an adequate size matrix such as, for every $K_{i}$ block, the matrix.

$A_{k}-K_{k} C_{k}$ should give all its eigenvalues with negative real part:

Let's suppose that there exists two integer sets $\left\{\sigma_{1}, \ldots, \sigma_{n} \in Z\right\}$ and $\left\{\delta_{1}>0, \cdots, \delta_{p}>0 \in N^{*}\right\}$ such as:

ii. $\sigma_{\mu_{k}+v}=\sigma_{\mu_{k}+v-1}+\delta_{r}, k=1, \cdots, p, v=1, \cdots, \eta_{k}-1$

iii. $\frac{\partial \varphi_{i}}{\partial z_{j}} \neq 0 \Rightarrow \sigma_{i} \geqslant \sigma_{j}, i, j=1, \cdots, n, j \neq \mu_{k}, k=1, \ldots, p$

So,

$$
\dot{\hat{z}}=A \hat{z}+\varphi(\hat{z}, u)-S_{\theta}^{-1} K(C \hat{z}-y)
$$

is an exponential observer for the system Eq. (13) as well.

And there exists $T_{1}$ such as, for all $T, 0<T<T_{1}$.

With,

$$
\begin{aligned}
& S(S, \delta)=\left[\begin{array}{lll}
S^{\delta_{1}} \Delta\left(S^{\delta^{1}}\right) & & \\
& \ddots & \\
& & S^{\delta_{p}} \Delta\left(S^{\delta^{p}}\right)
\end{array}\right] \\
& \Delta_{\theta}(S)=\left[\begin{array}{llll}
1 & & & \\
& S & & \\
& & \ddots & \\
& & & S^{\eta_{\theta}-1}
\end{array}\right]
\end{aligned}
$$


By operating a reverse variable change for coming back to the initial nonlinear system, the observer for the system Eq. (12) is given by:

$$
\dot{\hat{x}}=f(\hat{x})+g(\hat{x}) u-\left(\frac{\partial \Gamma}{\partial \hat{x}}(\hat{x}(t))\right)^{-1} S_{\theta}^{-1}(h(\hat{x})-y)
$$

$\hat{x}$ : Estimated value of $x$.

$\Gamma$ : An application $R^{n} \rightarrow R^{n}$.

With,

$\Gamma=\left[h_{1}, L_{f} h_{1}, L_{f}^{2} h_{1}, \ldots, L_{f}^{\delta_{1}} h_{1}, h_{2}, L_{f} h_{2}, L_{f}^{2} h_{2}, \ldots, L_{f}^{\delta_{2}} h_{2}, \ldots, h_{p}, L_{f} h_{p}, L_{f}^{2} h_{p}, \ldots, L_{f}^{\delta_{p}} h_{p}\right]^{T}$ And $L_{f}^{\delta_{k}}$ is the Lie $\delta_{k}^{i}$ derivative.

$P$ : Number of outputs.

And $S_{\theta}$ satisfies the following Lyapunov relation:

$$
\dot{S}=-\theta S_{\theta}-A^{T} S_{\theta}-S_{\theta} A+C^{T} C=0
$$

In [20], the demonstration is done.

\subsection{The unscented Kalman filter}

The unscented Kalman filter (UKF) has been essentially designed for the state estimation problems, and applied in some nonlinear control applications [11]. The unscented Kalman filter (UKF) compensates for approximation issues of the extended Kalman filter (EKF). A Gaussian random variable represents the state distribution, which is specified using a set of sample points chosen very carefully [12]. The unscented transformation (UT) is a method to estimate or calculate statistics of a random variable which is subjected to a nonlinear transformation [11]. In stochastic estimation problems, a common assumption usually is used which underline the fact that the process and measurement noise terms are additive, as in:

$$
\begin{aligned}
& x_{k}=f\left(x_{k-1}, u_{k-1}\right)+w_{k-1} \\
& y_{k}=h\left(x_{k}, u_{k}\right)+v_{k}
\end{aligned}
$$

The dimension of the sigma-points is the same as the state vector, that is to say $L=n_{x}$. The UKF is recursively executed, starting with the assumed initial conditions $\hat{x}_{0}$ and $P_{0}$. First a set of sigma-points are generated from the prior state estimate $\hat{x}_{k-1}$ and covariance $P_{k-1}$ at each discrete-time step, as in:

$$
\chi_{k-1}=\left[\hat{x}_{k-1} \hat{x}_{k-1}+\sqrt{L+\lambda} \sqrt{P_{k-1}} \hat{x}_{k-1}-\sqrt{L+\lambda} \sqrt{P_{k-1}}\right]
$$

For the next point, each sigma point is passed through the state prediction function $f$ that is nonlinear.

$$
\chi_{k, k-1}^{(i)}=f\left(\chi_{k-1}^{(i)}, u_{k-1}\right), \quad i=0,1,2, \ldots, 2 L
$$

$\chi_{k, k-1}$ means that this is the predicted value of the sigma-point based on the information from the prior time step. Sigma-points transformed, the post transformation mean and covariance are computed using weighted averages of the transformed sigma-points [21], 


$$
\begin{gathered}
\hat{x}_{k, k-1}=\sum_{i=0}^{2 L} \eta_{i}^{m} \chi_{k, k-1}^{(i)} \\
P_{k, k-1}=Q_{k-1}+\sum_{i=0}^{2 L} \eta_{i}^{c}\left(\chi_{k, k-1}^{(i)}-\hat{x}_{k, k-1}\right)\left(\chi_{k, k-1}^{(i)}-\hat{x}_{k, k-1}\right)^{T}
\end{gathered}
$$

where $\eta_{0}^{m}=\lambda /(L+\lambda)$ and $\eta_{0}^{c}=\lambda /(L+\lambda)+1-\alpha^{2}+\beta$. The measurement noise is also omitted from the observation function, as for the prediction as in:

$$
\psi_{k, k-1}^{(i)}=h\left(\chi_{k, k-1}^{(i)}, u_{k}\right)
$$

where is a matrix of output sigma-points. Output sigma-points are used to calculate output covariance matrix, the predicted output and cross-covariance by using:

$$
\begin{aligned}
\hat{y}_{k, k-1} & =\sum_{i=0}^{2 L} \eta_{i}^{m} \psi_{k, k-1}^{(i)} \\
P_{k}^{y y} & =R_{k}+\sum_{i=0}^{2 L} \eta_{i}^{c}\left(\psi_{k, k-1}^{(i)}-\hat{y}_{k, k-1}\right)\left(\psi_{k, k-1}^{(i)}-\hat{y}_{k, k-1}\right)^{T} \\
P_{k}^{x y} & =\sum_{i=0}^{2 L} \eta_{i}^{c}\left(\chi_{k, k-1}^{(i)}-\hat{x}_{k, k-1}\right)\left(\psi_{k, k-1}^{(i)}-\hat{y}_{k, k-1}\right)^{T}
\end{aligned}
$$

Due to the additive noise assumption, $R$ is added to the output covariance matrix. For calculating the Kalman gain matrix $K$, covariance matrices are used, using:

$$
K_{k}=P_{k}^{x y}\left(P_{k}^{y y}\right)^{-1}
$$

And then this Kalman gain matrix is used to update covariance estimates and the state, as in:

$$
\begin{aligned}
& \hat{x}_{k}=\hat{x}_{k, k-1}+K_{k}\left(y_{k}-\hat{y}_{k, k-1}\right) \\
& P_{k}=P_{k, k-1}-K_{k} P_{k}^{y y} K_{k}^{T}
\end{aligned}
$$

With $y_{k}$, the measurement vector, $\hat{x}_{k}$ is the a posteriori state and $P_{k}$ is the covariance estimates.

\subsection{The moving horizon estimation}

The moving horizon estimation is a powerful means of estimating the states, and having in particular the possibility to constrain the outputs, states and noises. We can be described it as a least-squares optimization that leads to a states' estimation and working with a limited amount of information. Its particularity is to avoid the recursive manner characteristic of the extended Kalman filter. Under different approaches, several researchers [22-28] studied it, however presenting many similarities. The moving and full state estimations almost follow the same steps. In the moving state estimation, variables can be handled contrary to the full state estimation. In the full state estimation, at current time $k$, all variables from initial time $n=0$ to $n=k$ are used in the calculation. With a horizon $H$, the moving state estimation uses in the calculation only the concerned variables (measured outputs, 
manipulated inputs and estimated states) from $n=k+1-H$ to $n=k$, a moving vectors collect them. First of all, consider the full state estimation problem. Let assume that the process can be represented by the following continuous-time model [29-31]:

$$
\dot{x}(t)=f(x(t), u(t))+G w(t)
$$

where $w_{k}$ is the control noise.

Where the Gaussian noise of zero mean is $w$. We can describe the measured outputs $y$ by the discrete-time model

$$
y_{k}=h\left(x_{k}\right)+v_{k}
$$

where $v_{k}$ is the observation noise.

The equivalent linear discrete model is given by:

$$
x_{k+1}=A x_{k}+B u_{k}+G w_{k}
$$

where the matrices $A$ and $B$ are the Jacobian matrices with respect to $f$ in relation to $x_{k}$ and $u_{k}$, respectively. The measurement model is linearized as:

$$
y_{k+1}=C x_{k+1}+v_{k+1}
$$

where the matrix $C$ is the Jacobian matrix of $h$ with respect to $x_{k}$. In the full state estimation problem, we have to minimize the following criterion with respect to the sequence of noises $\left\{w_{0}, \ldots, w_{k-1}\right\}$ and to the initial state $x_{0}$, and then the states $\hat{x}_{i}$ are obtained by using Eq. (28).

$$
J_{k}=\left(x_{0}-\hat{x}_{0}\right)^{T} \Pi_{0}^{-1}\left(x_{0}-\hat{x}_{0}\right)+\sum_{i=0}^{k-1}\left(v_{i+1}^{T} R^{-1} v_{i+1}+w_{i}^{T} Q^{-1} w_{i}\right)
$$

The weighting matrices $\Pi_{0}^{-1}, Q^{-1}$ and $R^{-1}$, respectively, symbolize the initial estimation, the confidence in the dynamic model and the measurements. The main disadvantage of full state estimation is that during the computation we notice the size of the optimization problem grows as time increases, and would likely cause a failure in the optimization. The favorable solution to this increasing size is to set the problem according to a moving-horizon approach.

Let us consider the problem of moving state estimation. The criterion Eq. (30) is split into two parts [24, 25]:

$$
J_{k}=J_{k-H}+\sum_{i=k-H}^{k-1}\left(v_{i+1}^{T} R^{-1} v_{i+1}+w_{i}^{T} Q^{-1} w_{i}\right)=J_{k-H}+J^{m h e}
$$

The second term $J_{m h e}$ of the criterion Eq. (31) depends on the sequence of noises $\left\{w_{k-H}, \ldots, w_{k-1}\right\}$ and on the state $x_{k-H}$. Assume that $k>H$ and set the optimized criterion:

$$
J_{k-H}^{*}=\min _{\mathrm{x}_{0}, w_{0}, \ldots, w_{k-H-1}} J_{k-H}
$$

And then, in the full optimized criterion becomes:

$$
J_{k}^{*}=\min _{\mathrm{x}_{0}, w_{0}, \ldots, w_{k-1}} J_{k}
$$




$$
=\min _{z, w_{k-H}, \ldots, w_{k-1}}\left[\sum_{i=k-H}^{k-1}\left(v_{i+1}^{T} R^{-1} v_{i+1}+w_{i}^{T} Q^{-1} w_{i}\right)\right]+J_{k-H}^{*}(z)
$$

where $z$ is the arrival state $x_{k-H}$ based on the optimized variables $\left\{w_{k-H}^{*}, \ldots, w_{k-H-1}^{*}\right\}$ and $x_{0}$.

In practice, it is very complicated and almost impossible to really minimize $J_{k-H}(z)$ when $k$ becomes large enough as this would be a full estimation problem again. The recommend solution is to retain the previous values of the optimized criterion $J_{k}^{*}$ obtained by moving horizon estimation denoted by $J_{k}^{\text {mhe }}(z)$ along time $k$ and to approximate $J_{k-H}(z)$ as:

$$
J_{k-H}(z) \approx\left(z-\hat{x}_{k-H}^{m h e}\right)^{T} \Pi_{k-H}^{-1}\left(z-\hat{x}_{k-H}^{m h e}\right)+J_{k-H}^{\text {mhe }}(z)
$$

where $\hat{x}_{k-H}^{\text {mhe }}$ is the state estimated by moving horizon observer at time $(k-H)$. Under these assumptions, the criterion Eq. (31) becomes:

$$
\begin{aligned}
J_{k}= & \sum_{i=k-H}^{k-1}\left(v_{i+1}^{T} R^{-1} v_{i+1}+w_{i}^{T} Q^{-1} w_{i}\right)+\left(z-\hat{x}_{k-H}^{\text {mhe }}\right)^{T} \Pi_{k-H}^{-1}\left(z-\hat{x}_{k-H}^{m h e}\right) \\
& +J_{k-H}^{m h e}(z)
\end{aligned}
$$

The discrete Riccati equation we used for the covariance matrix of the Kalman filter is called to update $\Pi_{k}$ :

$$
\Pi_{k}=A \Pi_{k-1} A^{T}+G Q G^{T}-A \Pi_{k-1} C^{T}\left[C \Pi_{k-1} C^{T}+R\right]^{-1} C \Pi_{k-1}^{T} A^{T}
$$

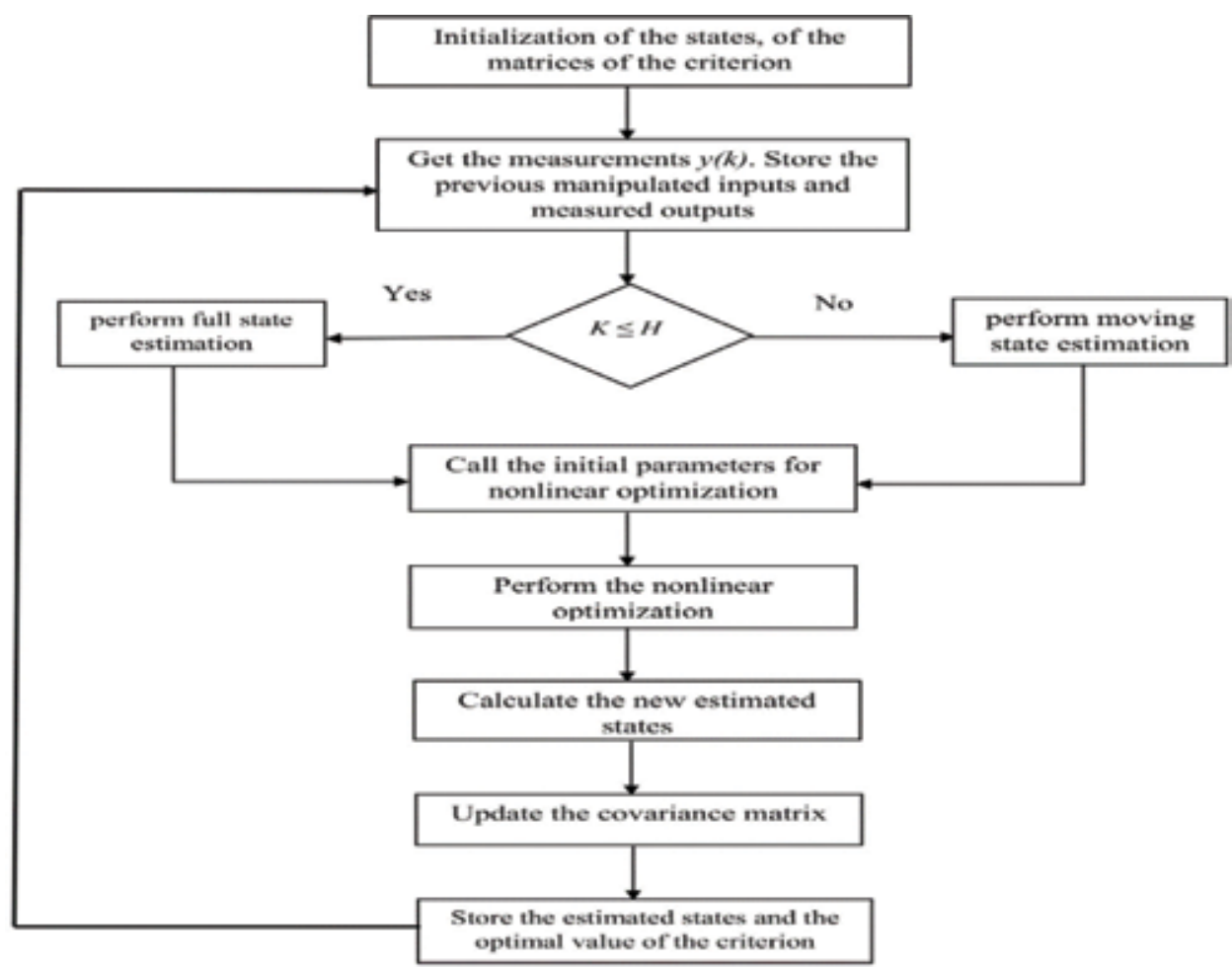

Figure 2.

Moving horizon estimation algorithm. 
With $\Pi_{0}$ given. The Moving horizon estimation algorithm is described by the diagram in Figure 2.

\section{Numerical results}

In this section, the performances of the proposed observers are illustrated in simulation. Observers' algorithms have been implemented in MATLAB/SIMULINK software. The doubly-fed induction generator system states which have been used for estimation are expressed into a vector $x$, this vector includes as parameters to estimate the stator and rotor resistances, as follows:

$$
x=\left[\begin{array}{llllll}
\Phi_{d s} & \Phi_{q s} & \Phi_{d r} & \Phi_{q r} & R_{s} & R_{r}
\end{array}\right]^{T}
$$

The inputs of the system are the rotor angular electrical speed, stator and rotor voltages, as in:

$$
u=\left[\begin{array}{lllll}
v_{d s} & v_{q s} & v_{d r} & v_{q r} & \omega_{r}
\end{array}\right]^{T}
$$

The $d$ and $q$ axis of stator and rotor currents and the mechanical torque constitute the measurements of the systems,

$$
y=\left[\begin{array}{lllll}
T_{m} & i_{d s} & i_{q s} & i_{d r} & i_{q r}
\end{array}\right]^{T}
$$

Table 3 shows a comparison of the running time of high gain observer (HGO), the unscented Kalman filter (UKF), and the moving horizon estimation (MHE) for the DFIG system. The high gain observer being the fastest among the three methods under various modes especially the healthy mode which represents a healthy DFIG and the faulty mode where stator and rotor resistance would have changed value during the operation of the DFIG. Tables 4 and 5 give the parameters of UKF and MHE only. For the UKF, the primary, secondary, and tertiary scaling parameters $\alpha$, $\beta$ and $\kappa$ are chosen as 1,2 , and 0 , respectively.

\begin{tabular}{llll}
\hline & HGO & UKF & MHE \\
\hline Healthy mode & 1.200 & 1.190 & 152.978 \\
\hline Faulty mode & 1.901 & 1.666 & 154.234 \\
\hline
\end{tabular}

Table 3.

Running time of the three observers for the DFIG (in seconds).

\begin{tabular}{lc}
\hline Parameters & Values \\
\hline Weight matrix G & eye $(6)$ \\
\hline Covariance matrix $\mathrm{P}_{0}$ & 3 eye $(6)$ \\
\hline Covariance matrix Q & 0.5 eye $(6)$ \\
\hline Covariance matrix R & eye $(5)$ \\
\hline Length horizon $\mathrm{H}$ & 10 \\
\hline Initial guess & {$[0 ; 0: 5 ; 0: 5 ; 1 ; 0: 02 ; 0: 02]$} \\
\hline
\end{tabular}




\begin{tabular}{lc}
\hline Parameters & Values \\
\hline Covariance matrix $P_{0}$ & eye $(6)$ \\
\hline Covariance matrix $Q$ & $10^{-2} \operatorname{diag}([111110-410-4])$ \\
\hline Covariance matrix $\mathrm{C}$ & $10^{-2} \operatorname{diag}([11111])$ \\
\hline Initial guess & {$[0 ; 0: 5 ; 0: 5 ; 1 ; 0: 02 ; 0: 02]$} \\
\hline
\end{tabular}

Table 5 .

UKF parameters.

Figures 3 and $\mathbf{4}$ show the generated estimates of the rotor and stator resistances by the HGO, UKF and the MHE in the healthy mode of working of the DFIG. Nevertheless, Figures 5 and $\mathbf{6}$ show the generated estimates of the rotor and stator resistances by the HGO, UKF and the MHE in the faulty mode of working, let us mention that faulty mode is simply a mode where the DFIG undergoes a fault on its stator and/or rotor resistances during the operation. We just simulated those scenarios to appreciate the estimation performance of different observers in particular the HGO, UKF and MHE for process monitoring or diagnostics purposes. We can observe that the estimates by the MHE converges to the actual parameters in fewer time compared to the HGO and UKF. In Table 3, we notice the total computation time to obtain an estimate for the HGO algorithm is about 1.200 seconds, for the UKF algorithm is also about 1.190 seconds while the MHE algorithm took 152.978 seconds to estimate the parameters in the normal mode of working, and in the faulty mode, we have about 1.901, 1.666 and 154.234 seconds for those observers, respectively. We can conclude that when the asynchronous machine has a stator or rotor resistance fault, the estimation time increases. The reason the MHE algorithm takes longer to make an estimate is that in simulation, the optimization of the objective function, through a nonlinear programming algorithm has been performed at each time step, in this case study the nonlinear programming algorithm used is the sequential quadratic programming in the MATLAB in-built function fmincon. For the HGO we can underline this, a big value of $\theta$ leads to consolidate the linear part and to guarantee the stability of the nonlinear part through the fact that $\varphi$ is imposed globally Lipschitz in relation to $x$ [27]. If $\theta$ are big enough, the time of convergence decreases, but the observation becomes extremely sensitive to the measurement noises. A small value of $\theta$ leads to the reverse effect obviously. In comparison with the extended Kalman filter, this observer contains a lot less of setting variables that facilitates its optimization. Besides the number of equations to solve are a lot weaker and it decreases the time of calculation considerably. To know that the number of differential equations to solve for the Kalman filter is of $n+\frac{n(n+1)}{2}$ such as, $n$ is the size of observation vector, when that number is $n$ for the high gain observer [18], for our experiment the value of the gain is $\theta=27$, on the other hand, the UKF algorithm has to handle.

$2 L+1$ sigma points and associated weights to represent state of the system. Tables 6 and 7 show the standard deviation and the variance of the estimation error. The comparison of these observers can be made by finding the mean squared error (MSE) value. The MSE can be evaluated as:

$$
M S E=\frac{1}{N \times n} \sum_{i=1}^{N}\left(\theta_{i}-\hat{\theta}_{i}\right)^{2}
$$

where $N$ is the number of time steps, $n$ is the dimension of state vector, $\theta_{i}$ is the simulated value and $\hat{\theta}_{i}$ is the estimated value from the filters. Table 8 shows a comparison of the three observers by finding the mean squared error in the healthy 
Wind Solar Hybrid Renewable Energy System

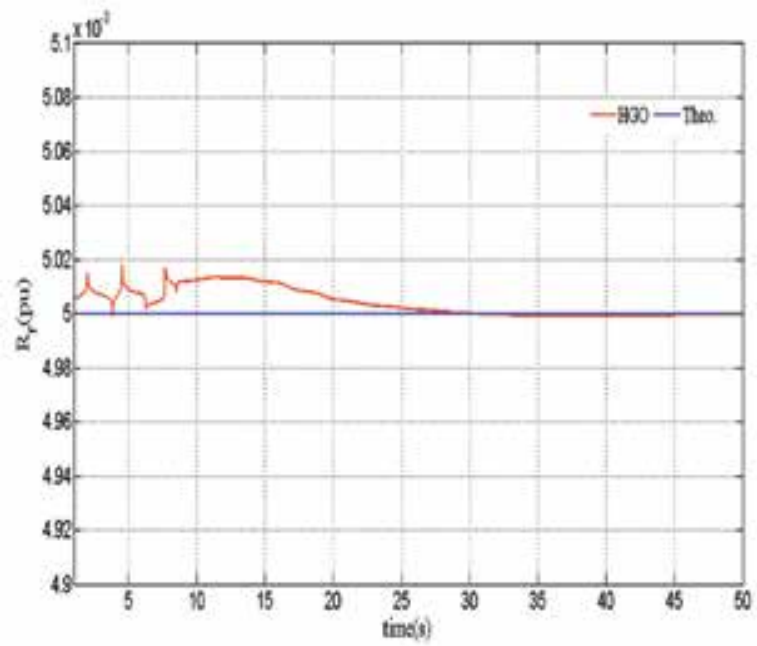

(a)

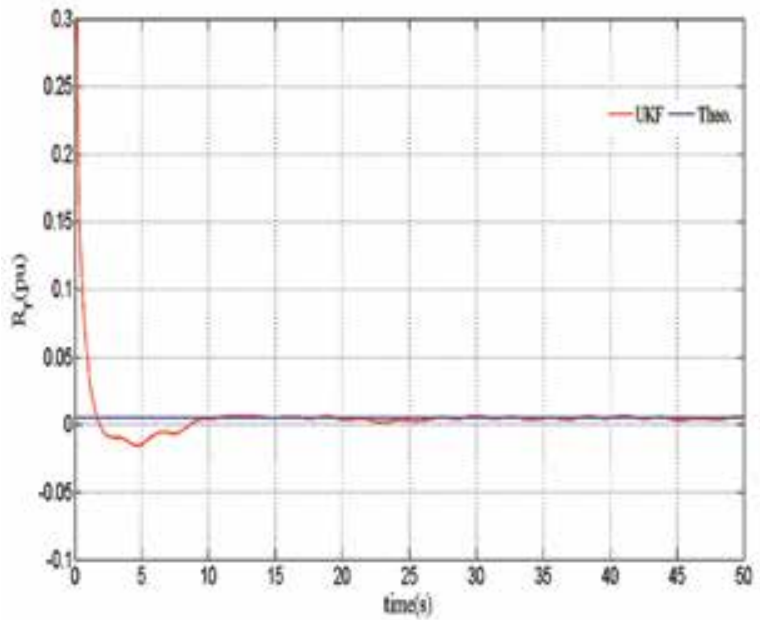

(b)

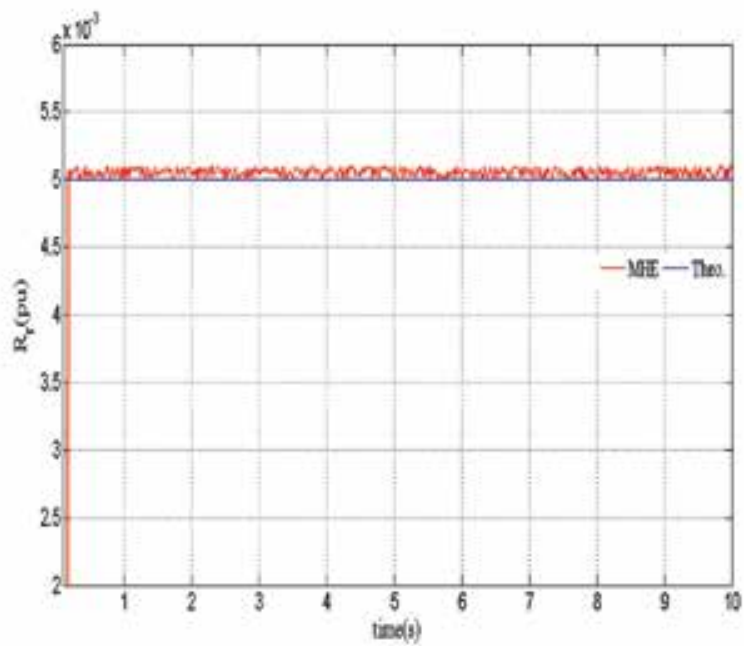

(c)

Figure 3.

Rotor resistance estimation in a healthy mode with HGO, UKF and MHE. (a) Rotor resistance estimation (HGO), (b) Rotor resistance estimation (UKF), (c) Rotor resistance estimation (MHE). 
Advanced Monitoring of Wind Turbine

DOI: http://dx.doi.org/10.5772/intechopen.84840

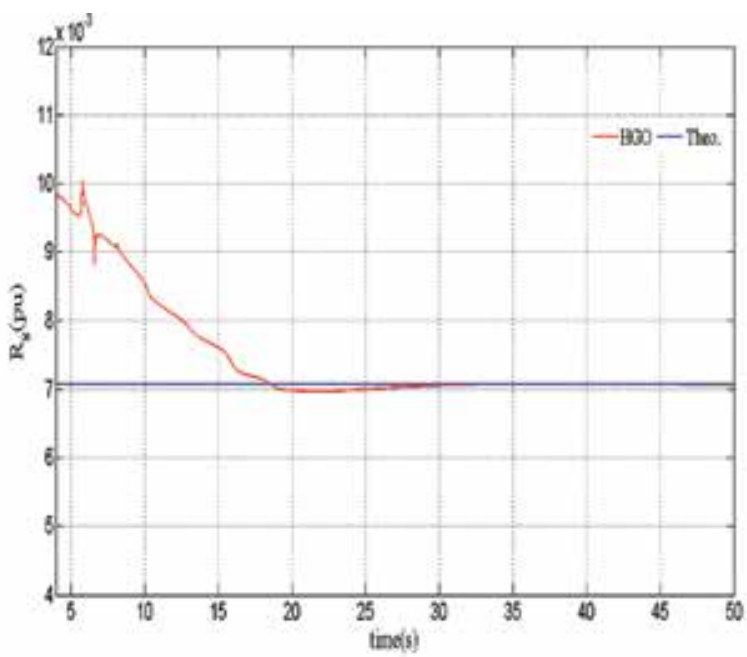

(a)

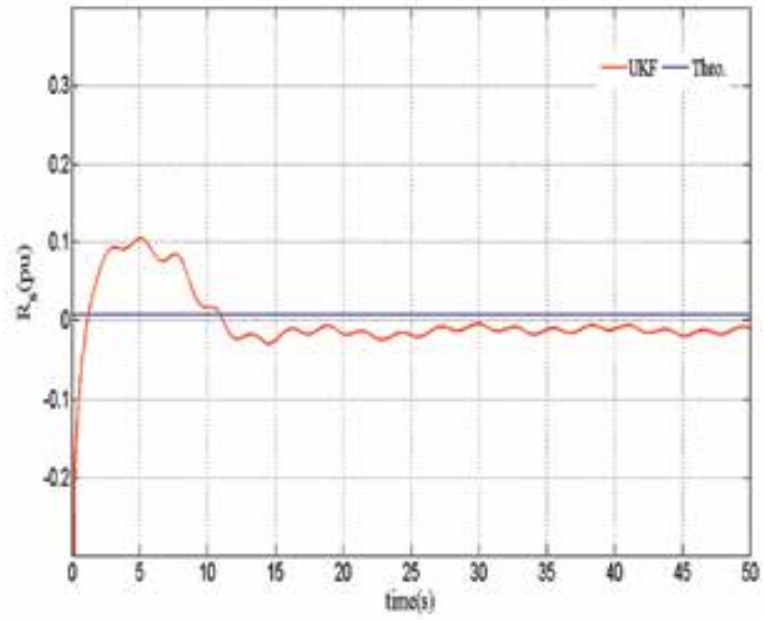

(b)

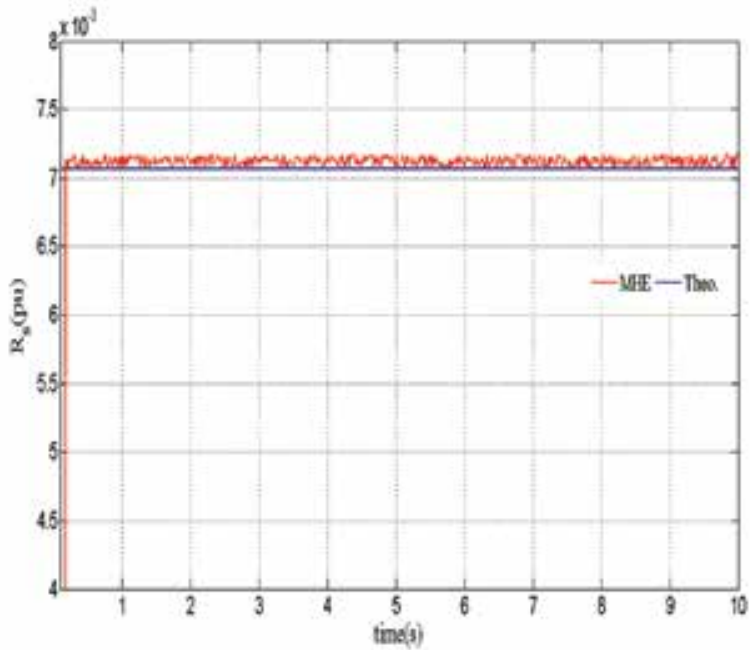

(c)

Figure 4.

Stator resistance estimation in a healthy mode with HGO, UKF and MHE. (a) Stator resistance estimation (HGO), (b) Stator resistance estimation (UFK), (c) Stator resistance estimation (MHE). 
Wind Solar Hybrid Renewable Energy System

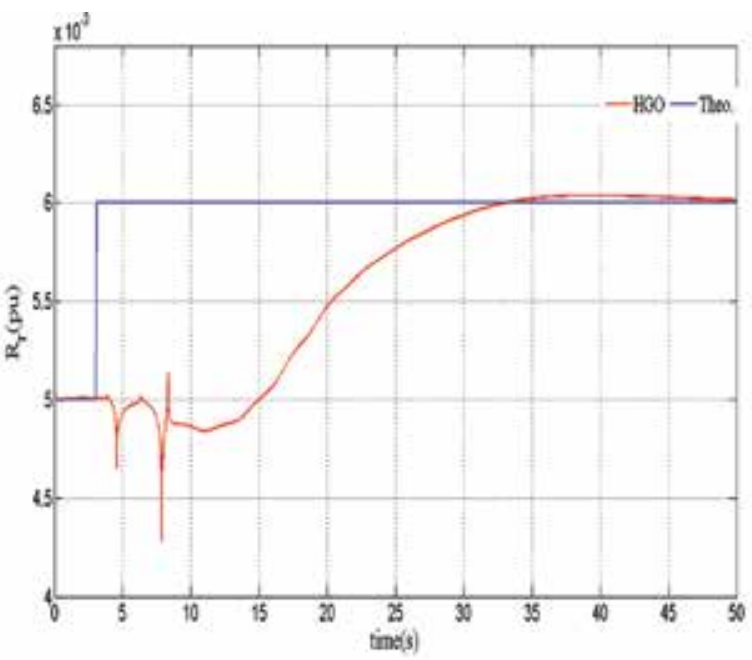

(a)

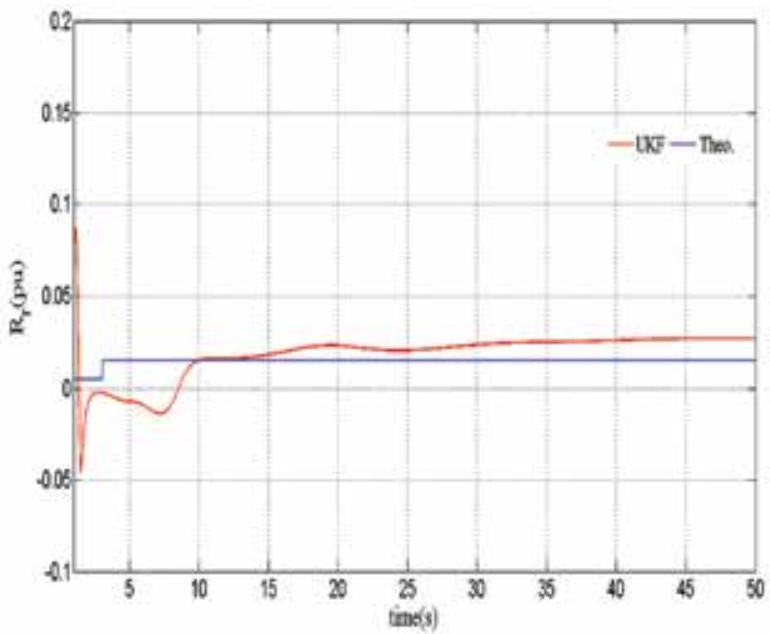

(b)

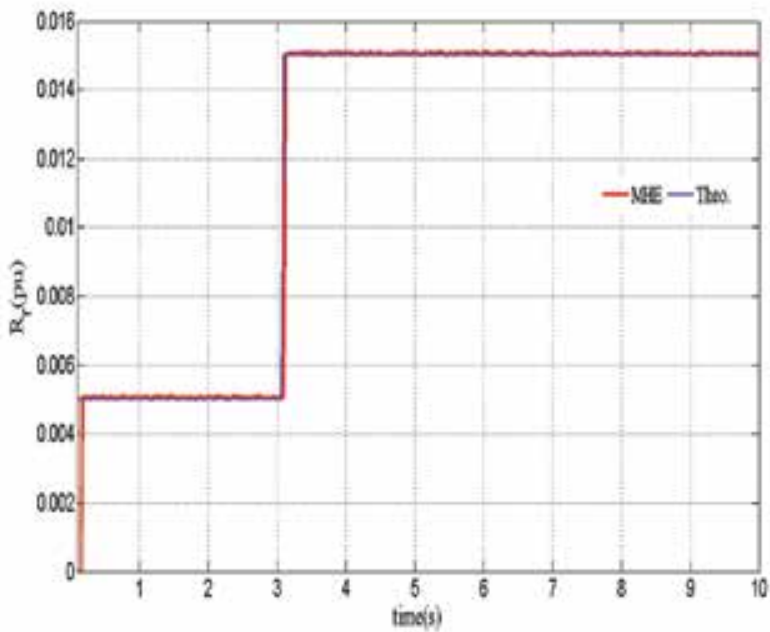

(c)

Figure 5.

Rotor resistance estimation in a faulty mode with HGO, UKF and MHE. (a) Rotor resistance estimation (HGO), (b) Rotor resistance estimation (UKF), (c) Rotor resistance estimation (MHE). 
Advanced Monitoring of Wind Turbine

DOI: http://dx.doi.org/10.5772/intechopen.84840

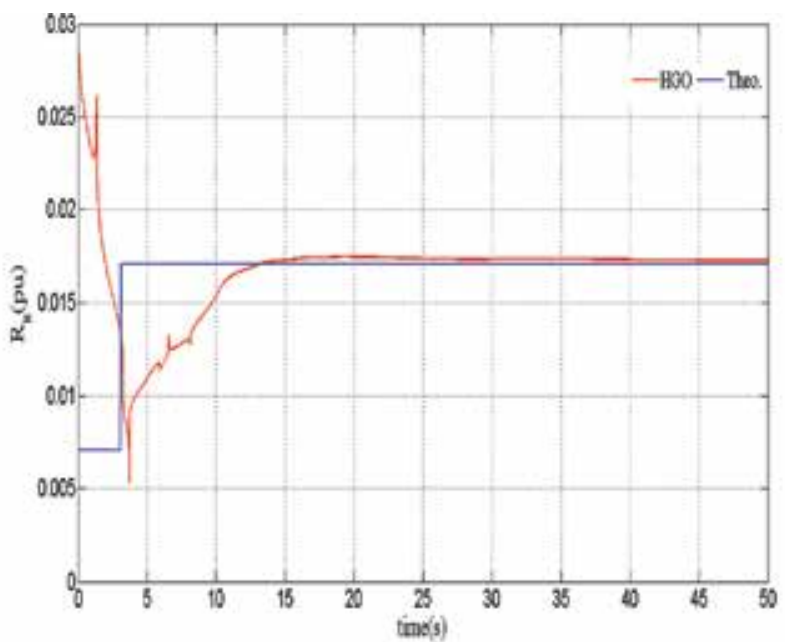

(a)

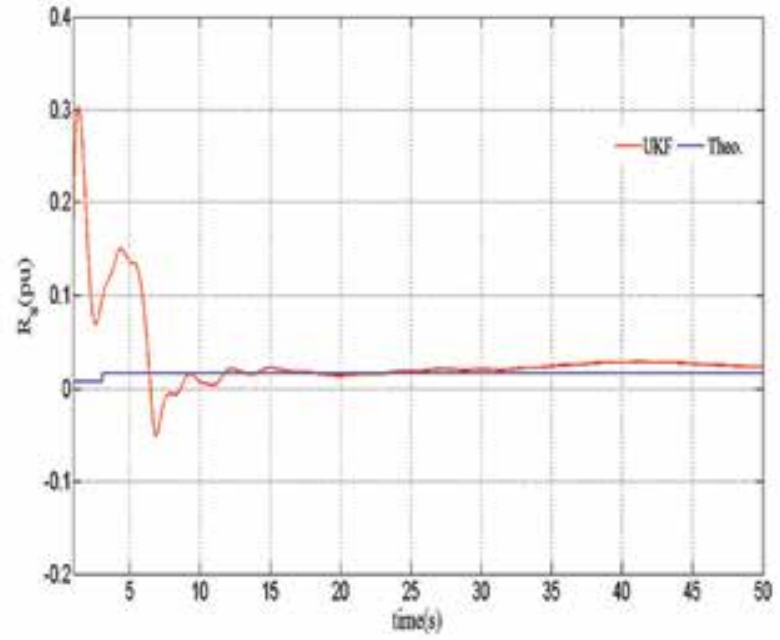

(b)

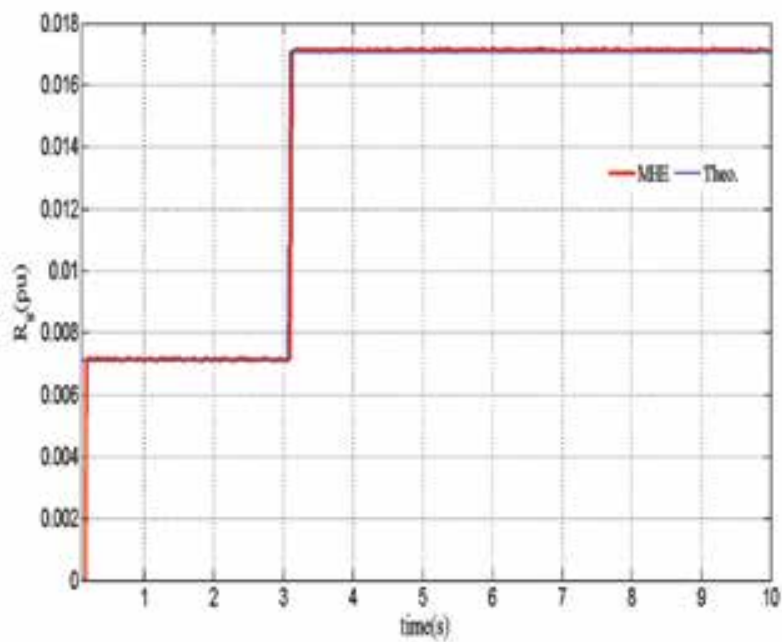

(c)

Figure 6.

Stator resistance estimation in a faulty mode with $H G O, U K F$ and MHE. (a) Stator resistance estimation (HGO), (b) Rotor resistance estimation (UKF), (c) Rotor resistance estimation (MHE). 


\begin{tabular}{cccc}
\hline & Std $(\mathbf{H G O}) \times \mathbf{1 0}^{-\mathbf{5}}$ & Std $($ UKF $) \times \mathbf{1 0}^{-\mathbf{5}}$ & Std $(\mathbf{M H E}) \times \mathbf{1 0}^{-\mathbf{5}}$ \\
\hline $\mathrm{Rs}$ & 350 & $\mathbf{7 4 5 0}$ & 57.75 \\
\hline $\mathrm{Rr}$ & 7.29 & 3940 & 41.38 \\
\hline & Variance $(\mathrm{HGO}) \times 10^{-5}$ & Variance $(\mathrm{UKF}) \times 10^{-5}$ & Variance $(\mathrm{MHE}) \times 10^{-5}$ \\
\hline $\mathrm{Rs}$ & 1.26 & 550 & 0.033 \\
\hline $\mathrm{Rr}$ & 0.0053 & 160 & 0.017 \\
\hline
\end{tabular}

Table 6.

General statistics of the three observers (healthy mode).

\begin{tabular}{cccc}
\hline & Std $(\mathbf{H G O}) \times \mathbf{1 0}^{-\mathbf{4}}$ & Std $($ UKF $) \times \mathbf{1 0}^{-\mathbf{4}}$ & Std $(\mathbf{M H E}) \times \mathbf{1 0}^{\mathbf{- 4}}$ \\
\hline $\mathrm{Rs}$ & $\mathbf{2 5}$ & 8208 & 270 \\
\hline $\mathrm{Rr}$ & 4.86 & $\mathbf{2 7 8}$ & 26 \\
\hline & Variance $(\mathrm{HGO}) \times 10^{-5}$ & Variance $(\mathrm{UKF}) \times 10^{-5}$ & Variance $(\mathrm{MHE}) \times 10^{-5}$ \\
\hline $\mathrm{Rs}$ & 0.62 & 6737 & 0.74 \\
\hline $\mathrm{Rr}$ & 0.024 & 77.47 & 0.702 \\
\hline
\end{tabular}

Table 7.

General statistics of the three observers (faulty mode).

\begin{tabular}{ccccccc}
\hline & \multicolumn{2}{c}{ HGO } & \multicolumn{2}{c}{ UKF } & \multicolumn{2}{c}{ MHE } \\
\hline & Healthy & Faulty & Healthy & Faulty & Healthy & Faulty \\
\hline $\mathrm{Rs}$ & $4.73 \mathrm{E}-06$ & $8.02 \mathrm{E}-06$ & $8.66 \mathrm{E}-04$ & $9.65 \mathrm{E}-04$ & $1.11 \mathrm{E}-07$ & $1.27 \mathrm{E}-07$ \\
\hline $\mathrm{Rr}$ & $1.77 \mathrm{E}-09$ & $1.79 \mathrm{E}-05$ & $9.36 \mathrm{E}-05$ & $1.14 \mathrm{E}-04$ & $5.71 \mathrm{E}-08$ & $7.33 \mathrm{E}-08$ \\
\hline$\Phi_{\mathrm{ds}}$ & $5.70 \mathrm{E}-04$ & $6.31 \mathrm{E}-04$ & $9.02 \mathrm{E}-05$ & $9.23 \mathrm{E}-05$ & $21.0 \mathrm{E}-04$ & $11.0 \mathrm{E}-04$ \\
\hline$\Phi_{\mathrm{qs}}$ & $1.93 \mathrm{E}-06$ & $2.10 \mathrm{E}-06$ & $1.10 \mathrm{E}-16$ & $1.10 \mathrm{E}-16$ & $39.0 \mathrm{E}-04$ & $27.0 \mathrm{E}-04$ \\
\hline$\Phi_{\mathrm{dr}}$ & $2.08 \mathrm{E}-08$ & $16.00 \mathrm{E}-04$ & $6.47 \mathrm{E}-06$ & $6.87 \mathrm{E}-06$ & $246 \mathrm{E}-04$ & $210 \mathrm{E}-04$ \\
\hline$\Phi_{\mathrm{qr}}$ & $1.73 \mathrm{E}-10$ & $4.81 \mathrm{E}-06$ & $1.10 \mathrm{E}-06$ & $1.10 \mathrm{E}-06$ & $67.0 \mathrm{E}-04$ & $70.0 \mathrm{E}-04$ \\
\hline
\end{tabular}

Table 8.

MSE values of nonlinear observers: HGO, UKF and MHE are compared.

and the faulty mode of operation of the DFIG and we can notice that generally, the mean squared error of states and parameters in faulty mode is relatively greater than those in the healthy mode because of the fault occurring suddenly during the operation, but we can always see the high performance of the moving horizon estimation on the others observers.

To verify the robustness, we have performed parametric variation on the observer in relation to the identified values. Figures 7 and 8 show the responses obtained when a rotor inductance variation of +50 and $-50 \%$ is considered for the observer test. The robustness of the observers' scheme with respect to this parameter changes is clearly shown. In Figures 7 and $\mathbf{8}$, it is clearly shown that $a+50$ and $-50 \%$ rotor inductance variation generates a high statistical difference on rotor and stator resistances for the unscented Kalman filter. For the high gain observer, that variation is much more felt on the rotor resistance on the both figures. Incontestably the moving horizon estimation seems remain insensitive to the parametric 
variations but it is not so, it is just that the statistical difference generated is weak enough compared to others. From these responses, we can conclude that the rotor inductance changes do not affect the performance of the moving horizon estimation

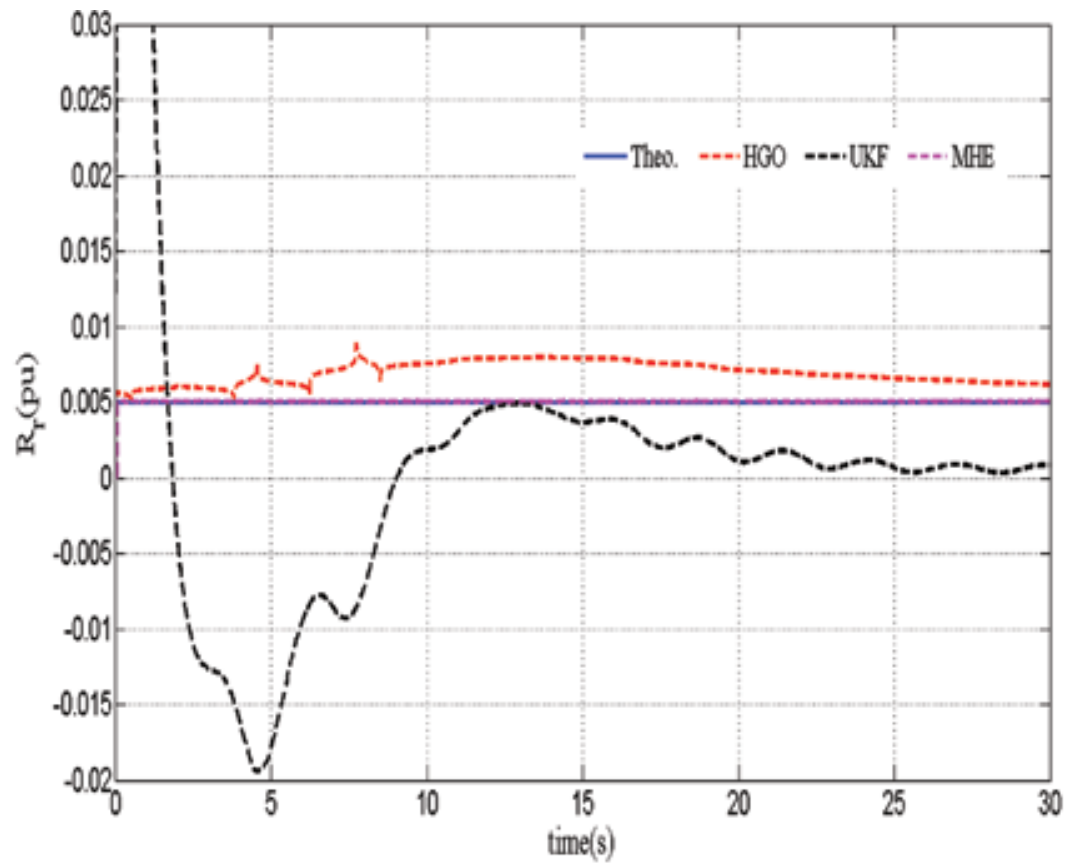

(a)

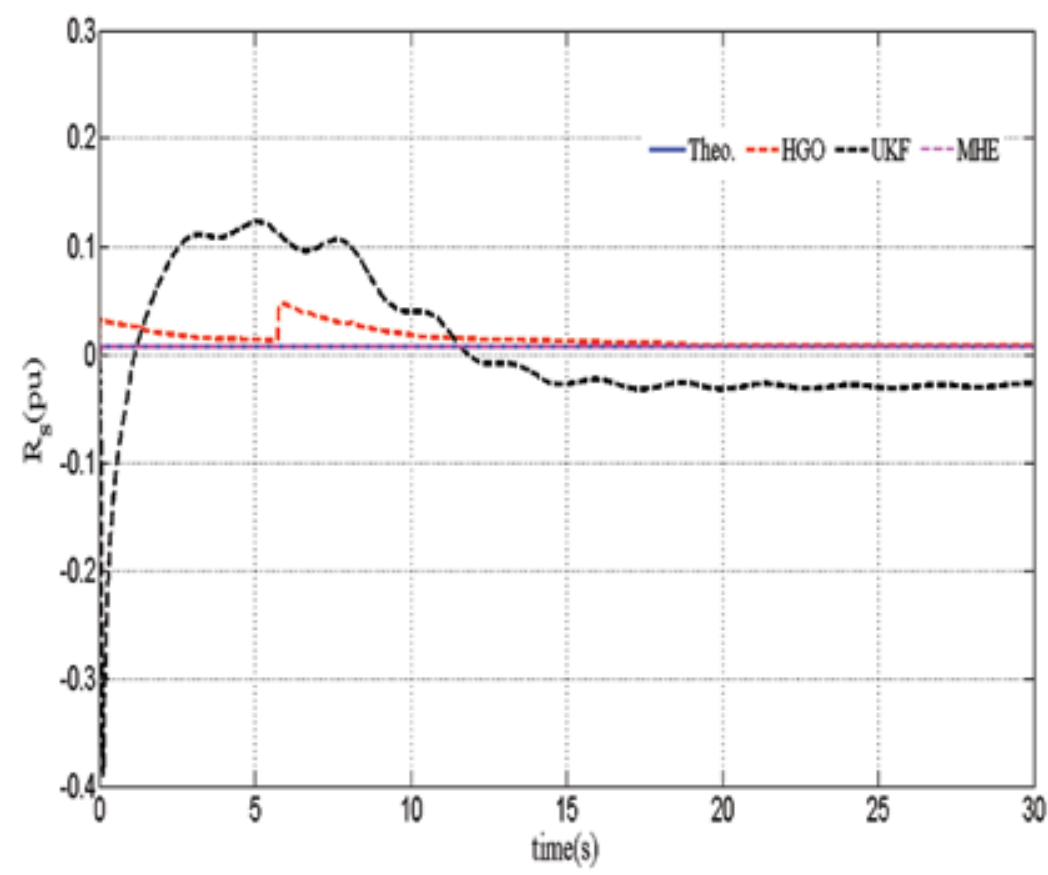

(b)

Figure 7.

Robustness test. Rotor inductance variation ( $+50 \%)$. (a) Rotor resistance estimation, (b) Stator resistance estimation. 


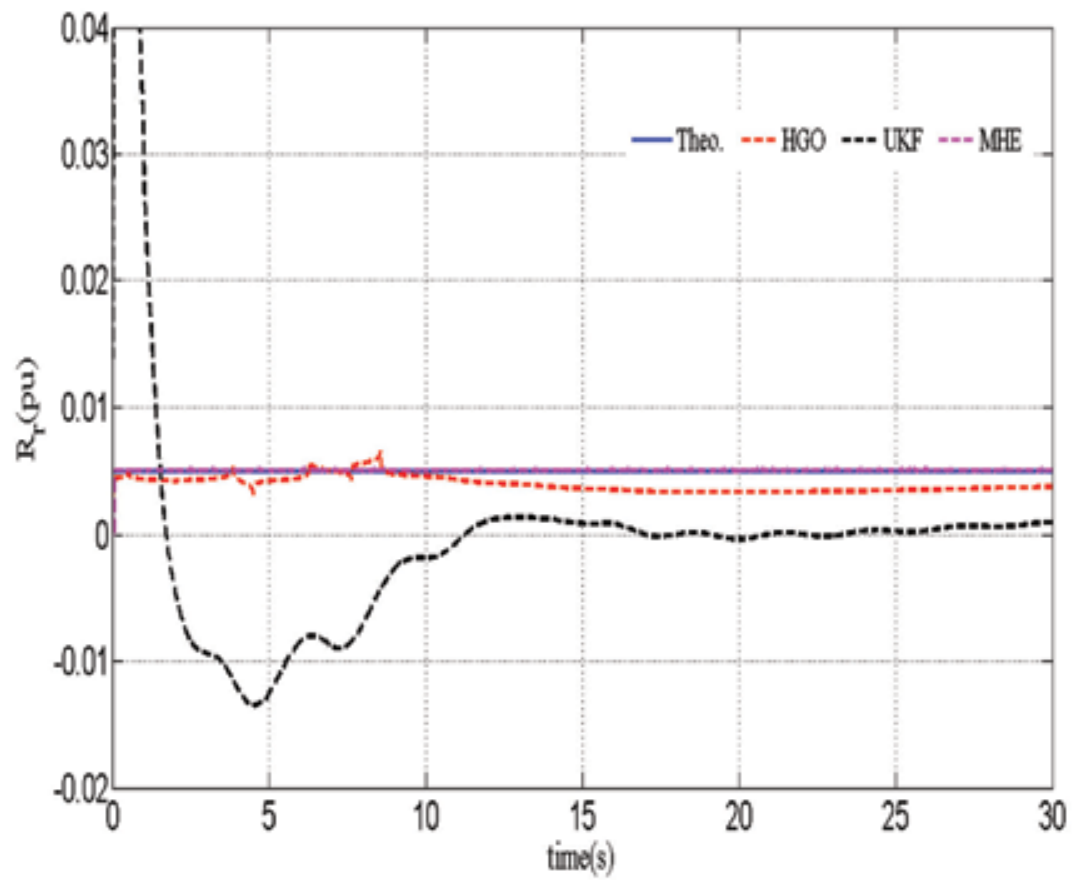

(a)

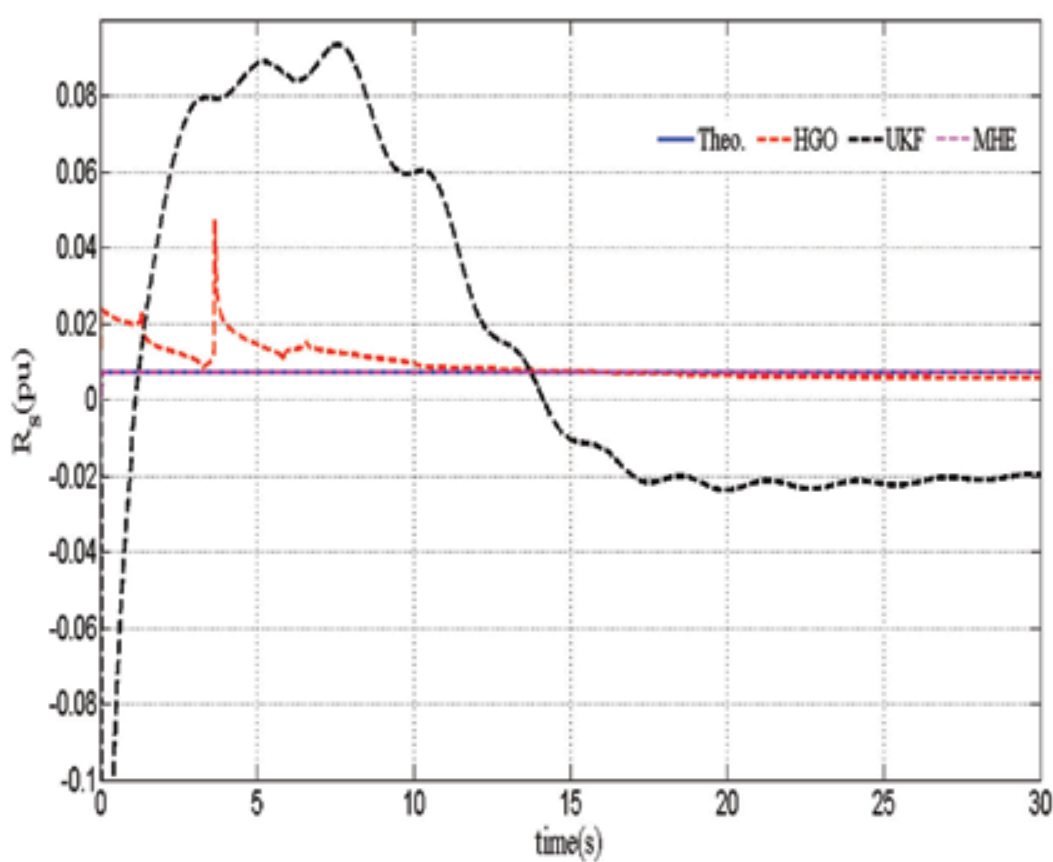

(b)

Figure 8.

Robustness test. Rotor inductance variation (-50\%). (a) Rotor resistance estimation, (b) Stator resistance estimation.

considerably, but in regard to the other observers, the changes disturb their performance a lot as shown in the figures and that the MHE scheme is robust enough under parametric uncertainties. 


\section{Conclusion}

In this chapter, a general framework for the doubly fed induction generator has been presented in order to carry out a dynamic estimation of states and parameters of the DFIG. The DFIG parameters are largely influenced by different factors (for instance, temperature, magnetic saturation and eddy current) that is why it is necessary to develop techniques to estimate the changes of parameters. The proposed techniques are performed with high gain observer (HGO), unscented Kalman filter (UKF) and moving horizon estimation algorithms using noisy measurements. A comparison of the three estimation techniques has been made under different aspects notably, computation time and estimation accuracy, in two modes of operation of the DFIG, the healthy mode and the faulty mode. The MHE estimation technique has significantly lower estimation error and converges with fewer samples time than the HGO and the UKF. Whatever the mode of functioning, the simulation results showed that a good standard of performance could be obtained even in the presence of measurement noise.

\section{Author details}

Steve Alan Talla Ouambo, Alexandre Teplaira Boum*

and Adolphe Moukengue Imano

Department of Physics, Faculty of Science, University of Douala, Cameroon

*Address all correspondence to: boumat2002@yahoo.fr

\section{IntechOpen}

(C) 2019 The Author(s). Licensee IntechOpen. This chapter is distributed under the terms of the Creative Commons Attribution License (http://creativecommons.org/licenses/ by/3.0), which permits unrestricted use, distribution, and reproduction in any medium, provided the original work is properly cited. (cc) BY 


\section{References}

[1] Tazil M, Kumar V, Bansal RC, Kong S, Dong ZY, Freitas W, et al. Threephase doubly fed induction generators: An overview. IET Electric Power Applications. 2010;4(2):75-89

[2] Marinelli M, Morini A, Pitto A, Federico S. Modeling of doubly fed induction generator (DFIG) equipped wind turbine for dynamic studies. In: 2008 43rd International Universities Power Engineering Conference. Padova, Italy: IEEE; 2008. pp. 1-6

[3] Shenglong Y, Emami K, Fernando T, Herbert HCI, Wong KP. State estimation of doubly fed induction generator wind turbine in complex power systems. IEEE Transactions on Power Systems. 2016;31(6):4935-4944

[4] Pena R, Clare JC, Asher GM. Doubly fed induction generator using back-toback PWM converters and its application to variable-speed windenergy generation. IEE ProceedingsElectric Power Applications. 1996; 143(3):231-241

[5] Ledesma P, Usaola J. Doubly fed induction generator model for transient stability analysis. IEEE Transactions on Energy Conversion. 2005;20(2):388-397

[6] Rothenhagen K, Fuchs FW. Current sensor fault detection and reconfiguration for a doubly fed induction generator. In: 2007 IEEE Power Electronics Specialists Conference. Orlando: IEEE; 2007. pp. $2732-2738$

[7] Campos-Delgado DU, EspinozaTrejo DR, Palacios E. Fault-tolerant control in variable speed drives: A survey. IET Electric Power Applications. 2008;2(2):121-134

[8] Abdelmalek S, Rezazi S, Azar AT. Sensor faults detection and estimation for a dfig equipped wind turbine.

Energy Procedia. 2017;139:3-9

[9] Rothenhagen K, Fuchs FW. Modelbased fault detection of gain and offset faults in doubly fed induction generators. In: 2009 IEEE International Symposium on Diagnostics for Electric Machines, Power Electronics and Drives. Cargese: IEEE; 2009. pp. 1-6

[10] Li D, Lin X, Hu S, Kang Y. An adaptive estimation method for parameters of doubly-fed induction generators (DFIG) in wind power controller. In: 2010 Asia-Pacific Power and Energy Engineering Conference. Chengdu: IEEE; 2010. pp. 1-4

[11] Julier SJ, Jeffrey KU. New extension of the Kalman filter to nonlinear systems. In: Signal Processing, Sensor Fusion, and Target Recognition VI. Vol. 3068. International Society for Optics and Photonics; 1997. pp. 182-194

[12] Wan EA, Van Der Merwe R. The unscented Kalman filter for nonlinear estimation. In: Proceedings of the IEEE 2000 Adaptive Systems for Signal Processing, Communications, and Control Symposium. IEEE; 2000. pp. 153-158

[13] Dida A, Attous DB. Doubly-fed induction generator drive based WECS using fuzzy logic controller. Frontiers in Energy. 2015;9(3):272-281

[14] Pan X, Ju P, Wu F, Jin Y.

Hierarchical parameter estimation of DFIG and drive train system in a wind turbine generator. Frontiers of Mechanical Engineering. 2017;12(3): 367-376

[15] Heier S. Grid integration of wind energy: onshore and offshore conversion systems. John Wiley \& Sons; 2014. pp. 31-117 
[16] Leonhard W. Controlled ac drives, a successful transition from ideas to industrial practice. Control Engineering Practice. 1996;4(7):897-908

[17] Yang L, Xu Z, Østergaard J, Dong $\mathrm{ZY}$, Wong KP, Ma X. Oscillatory stability and eigenvalue sensitivity analysis of a DFIG wind turbine system. IEEE Transactions on Energy Conversion. 2011;26(1):328-339

[18] Boum AT, Talla SA. High gain observer and moving horizon estimation for parameters estimation and fault detection of an induction machine: A comparative study. Journal of Control and Instrumentation. 2017;15-26(08):8

[19] Nijmeijer H, Fossen TI. New Directions in Nonlinear Observer Design. Vol. 244. Springer; 1999

[20] Bornard G, Hammouri H. A high gain observer for a class of uniformly observable systems. In: Proceedings of the 30th IEEE Conference on Decision and Control. Brighton, UK: IEEE; 1991. pp. 1494-1496

[21] Rhudy M, Gu Y. Understanding nonlinear Kalman filters. Part II: An implementation guide. Interactive Robotics Letters; 2013. p. 1-18

[22] Corriou J-P. Process Control. Springer-Verlag; 2004

[23] Michalska H, Mayne DQ. Moving horizon observers and observer-based control. IEEE Transactions on Automatic Control. 1995;40(6): 995-1006

[24] Rao CV, Rawlings JB, Lee JH. Constrained linear state estimation-A moving horizon approach. Automatica. 2001;37(10):1619-1628

[25] Robertson DG, Lee JH, Rawlings JB. A moving horizon-based approach for least-squares estimation. AICHE Journal. 1996;42(8):2209-2224
[26] Slotine J-JE, Hedrick JK, Misawa EA. On sliding observers for nonlinear systems. Journal of Dynamic Systems, Measurement, and Control. 1987; 109(3):245-252

[27] Gauthier JP, Hammouri H, Othman S. A simple observer for nonlinear systems applications to bioreactors. IEEE Transactions on Automatic Control. 1992;37(6):I875

[28] Choqueuse V, Benbouzid M. Induction machine faults detection using stator current parametric spectral estimation. Mechanical Systems and Signal Processing. 2015;52:447-464

[29] Liu K, Zhu ZQ. Position offsetbased parameter estimation for permanent magnet synchronous machines under variable speed control. IEEE Transactions on Power Electronics. 2015;30(6):3438-3446

[30] Smith AN, Gadoue SM, Finch JW. Improved rotor flux estimation at low speeds for torque MRAS-based sensorless induction motor drives. IEEE Transactions on Energy Conversion. 2016;31(1):270-282

[31] Alonge F, Cirrincione M, Pucci M, Sferlazza A. Input-output feedback linearization control with on-line MRAS-based inductor resistance estimation of linear induction motors including the dynamic end effects. IEEE Transactions on Industry Applications. 2016;52(1):254-266 



\title{
Modeling and Simulation of a $10 \mathrm{~kW}$ Wind Energy in the Coastal Area of Southern Nigeria: Case of Ogoja
}

\author{
Gabriel Modukpe and Don Diei
}

\begin{abstract}
This research demonstrates a model of a wind energy conversion system that operates at different wind speed, with results simulated in MATLAB SIMULINK. The wind turbine system is made up of three parts or subsystems namely the aerodynamic, mechanical and electrical blocks. The system is designed by modeling differential equations for each block and then simulated in SIMULINK environment. The Squirrel Case Induction Generator, horizontal axis wind turbine system with complexities of all three parts of the wind turbine model were analyzed using the mathematical equations, with each block modeled and designed separately, then all three blocks joined together to give the complete unit. Wind speed data from Ogoja community in southern part of Nigeria was used to test the simulation performance. The system simulation was tested and worked satisfactorily, with different wind speed giving proportionate mechanical torque and turbine speed. This model therefore demonstrates that wind energy could be extracted in the region even at varying wind speed.
\end{abstract}

Keywords: modeling, simulation, wind energy, Southern Nigeria, coastal area, Ogoja

\section{Introduction}

Demand for renewable energy has been increasingly justified over the last couple of years as world powers turn to clean, green energy while overdependence on fossil fuel generation is still prominent in developing countries. Nevertheless, there is an expectation that renewable energy sources will play a wider role over the next two decades in energy with wind energy projected to contribute 1.1 trillion kilowatt-hours $(\mathrm{kWh})$ of a total of close to 4 trillion $\mathrm{kWh}$ of renewable energy expected to be generated by year 2030. Furthermore, research suggests that solar and wind energy are currently the most likely to provide economically affordable alternate energy sources, because other renewable energy sources like tidal remain costly and inefficient [1]. It is obvious that wind and solar energy studies will be the center of future renewable engineering efforts. Solar energy is becoming more dominant especially in the Arab world where their massive deserts have already seen investigations into the possibility of generating solar energy with enough 
capacity to power entire countries. With wind energy however, developing offshore wind turbines fit for tackling the substantially higher wind speeds accessible offshore while avoiding the issues of horizon and noise pollution is the way to go. In Ogoja community of southern Nigeria, the average wind speed is seen in this research to be good enough for a horizontal axis wind turbine using a Squirrel Cage Induction Generator due to its seeming advantages as detailed in later sections. Modern offshore wind energy systems are now faced with expectations of generating highly efficient, network frequency electricity in an autonomous and programmed manner and for 20 or more years consistently and continuously with little or no maintenance requirements in some of the harshest environments in the world. This constitutes the challenges encountered by wind energy engineers today [2].

\section{Problem statement}

Natural gas is being used in gas turbines as a means of power generation in most countries, majority of which are in Africa. The effects of these fuels in the atmosphere led to the need for green energy, a clean and sustainable alternate source of energy generation. Different countries are facing devastating effects from the climate change, stormy rain, hurricane, great flood, etc., leading to different countries conducting research in renewable energy sources which will produce power without damaging the environment. Wind energy is abundant on the Earth and has a low or no impact in environmental pollution. The energy is generated naturally from wind; hence fuel needed for wind turbines is free and occurs every day. This study shows a wind energy system modeled and runs at various wind speeds, similar to wind speeds found in some parts of the world.

\section{Aim and objectives of study}

The aim of the project is to design with the aid of mathematical modeling a wind energy conversion system that will produce energy at varying speed and test results using simulations. The following are the objectives:

- Model a wind energy conversion system by using mathematical equations.

- Simulate the system design using MATLAB SIMULINK Software, version R2017a.

- Test to see the effects of varying wind speeds.

- Simulate the aerodynamic, mechanical and electrical component design.

\section{Wind energy}

Wind energy is the indirect form of solar energy which is always being replenished by the sun [4]. The energy conversion occurs when electrical power or electricity is generated using the abundant natural resource wind. A wind turbine is an energy conversion device that changes the wind's kinetic energy into electrical energy. The operation of wind turbines makes use of the turning of two or three propeller-like blades around a rotor by wind. The rotor is attached to the main shaft, 
which turns a generator to create electricity [5]. The quantitative measurement of accessible wind energy at any point is called the wind power density (WPD). It is calculated as available mean power per square meter of area swept by a turbine with SI unit of watt per square meter. This indicates how much extractable energy on site.

Wind turbines are fabricated in two axis types (vertical and horizontal) and in a wide variety. The smallest types of wind turbines are used as a means of charging battery units used for generation of back-up power. Larger turbines are used to generate power for domestic use. Wind power can be classified as:

- Utility scale wind systems; wind systems that generate power larger than 100 kilowatts $(\mathrm{kW})$ to provide power to a grid system.

- Distributed or small wind systems, which uses wind turbines of $100 \mathrm{~kW}$ or lower than that to power directly a home, farm, etc.

- Offshore wind systems, are turbines mounted on water bodies around the world. Depending on speed of wind in that area, they can be used to power whole communities.

\subsection{Wind energy in Nigeria}

Wind energy is of course, one of the cheapest renewable sources per unit of energy produced, as well its technologies is one of the fastest rising technologies in energy generation industry across the world, yet not so much in Nigeria and SubSaharan Africa. It has been suggested that a network of land based 2.5 MW wind turbines can generate over 40 times the current electricity consumption in the world [5]. In Nigeria, renewable energy sources have been restricted to solar energy this is because wind energy is not considered viable due to low wind speeds in most parts of the country. Wind speed is generally considered moderate in the south with the exception of coastal areas and offshore. On the other hand, in the hilly regions of the north, it is strongest [6].

An analysis of wind energy potential in Kano State, Nigeria was done by [7], based on wind data taken for 21 years at a height of $10 \mathrm{~m}$. The data was statistically tested using Weibull probability density function. Results showed an expected average wind speed ranging from 6.5 to $9 \mathrm{~m} / \mathrm{s}$, good enough to drive a wind conversion system with wind power estimations as high as $12 \mathrm{MWh} / \mathrm{m}^{2}$. Five practical wind turbines where also analyzed with the data, giving positive results and economic viability of wind power in Kano State. Refs. [8, 9] noted the viability of renewable energy in Nigeria, the advantages and challenges and as well stated that the high cost of power supply and carbon emission reduction could be realized with the use of renewable sources energy.

In another article on wind energy potential in selected south western states, the investigation surveyed wind energy capability often chosen sites in the south western region of Nigeria and carried out a cost benefit analysis at those sites. Wind speed data at $10 \mathrm{~m}$ height gotten from the Nigerian Meteorological Agency was utilized to classify the sites wind profiles for electricity generation. The result demonstrated that sites in Lagos and Oyo States were suited for generation at a substantial scale with average wind speeds. Enough power can be generated with several small turbines connected together. The result demonstrated that the region's wind profiles and qualities are reasonable enough for wind power generation. Average wind speeds from 1.9 to $5.3 \mathrm{~m} / \mathrm{s}$ are predominant, while the most likely wind speed ranged between 1.9 and $6.2 \mathrm{~m} / \mathrm{s}$, with the maximum energy conveying speeds between 2.2 and $8.6 \mathrm{~m} / \mathrm{s}$ across all the stations [7]. 
Ref. $[10,11]$ conducted a research and reported on the wind energy reserve in Nigeria at $10 \mathrm{~m}$ ( or $40 \mathrm{~m}$ ) height based on data analysis on 10 wind stations across the North West, North East, North Central, South East and South West geopolitical zones. The research showed some promise, with some sites having wind regime between 3.6 and $5.1 \mathrm{~m} / \mathrm{s}$, therefore confirming that Nigeria falls into the moderate wind regime according to the Beaufort scale. Along these lines it can be inferred that the sites are potential wind farm areas. This is because most wind turbines start generating electricity at wind speeds of around 3-4 m/s, known in wind generation as the cut in speed. The report also suggested that Nigerian shoreline areas from Lagos State through Ondo, Delta, Rivers, Bayelsa to Akwa-Ibom States also showed promising potentials for harvesting moderate wind energy throughout the year. Coastal regions constitute majority of oil and gas activities in the country, with these activities causing environmental degradation while some of these communities are also cut off from the electricity grid hence leading to a quest for alternate energy sources.

\subsection{History of wind energy for wind farms}

Arrays of large turbines, called wind farms, are utilized to generate power as a means of reducing fossil power generation in developed countries. By the start of the twentieth century in Denmark, there were already in subsistence some 2500 windmills used to drive mechanical loads like grinding mills and water pumps with an estimated total peak power in the region of $30 \mathrm{MW}$. By 1910 there were electric generators ranging in power from 5 to $25 \mathrm{~kW}$ driven by wind and in use in the United States. During World War I, windmill engineers in the United States were manufacturing 100,000 small-scale farm windmills yearly, mostly used as water pumps [12]. One of the very first modern design horizontal-axis wind generators was used in the Soviet Union by 1931. It was a $100 \mathrm{~kW}$ generator placed on a $30-\mathrm{m}$ tall tower and connected to the Nation's $6.3 \mathrm{kV}$ electricity distribution system. It was accounted for to have had a yearly capacity factor of about $32 \%$, which shares close similarity to the efficiency exhibited by current wind machines [12]. As stated earlier, turbine blades can spin about a horizontal or a vertical axis, with horizontal axis rotation being older and more popular. They can also come with blades or be bladeless. Vertical axis wind turbines are not used as much because they produce less power [3].

\section{Types of wind turbine}

Considering how the turbine spins, two kinds of wind turbines can be defined. The mechanism is the same only the direction of the spin differs. Wind turbines that rotates along its vertical axis is the vertical axis wind turbines (VAWT), while the ones that spins about a horizontal axis is the horizontal axis wind turbines (HAWT).

\subsection{Horizontal axis wind turbines}

The horizontal axis wind turbine (HAWT) is a turbine whose rotor rotational axis is parallel to the ground and wind stream [13]. Its primary rotor shaft and electrical generator are at the pinnacle of the tower and must be faced directly to the wind. Micro turbines are directed by a wind vane, with larger turbines utilizing a wind sensor coupled with a servomotor. The gear box is located in the drive train and is used to convert the slow blade movement into much quicker rotation capable 


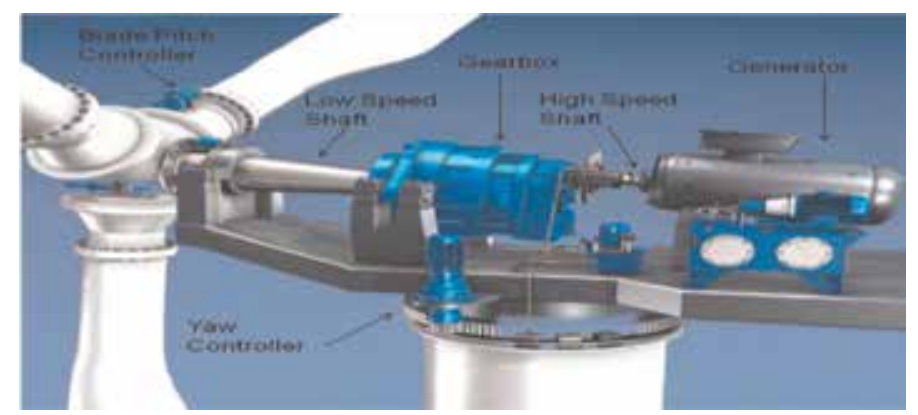

Figure 1.

A horizontal axis wind turbines [30].

of enough energy to drive an electrical generator [14]. Most HAWTs are either two or three blades, but the number of blades has no limit, it depends solely on the designer. HAWT could also be classified as upwind and downwind turbine. In Ref. [15] it is stated that a gear system is used for stepping up the speed of the generator, although designs may likewise utilize an annular generator. Some designs operate at fixed speed, but variable speed turbines have better efficiency and employ a power converter to communicate with the transmission system. All turbines come with protective lineaments for damage limitation during turbulence. In such turbulence the system is also controlled by feathering the blades into the wind hence stalling them, and brought to a halt with the aid of brakes (Figure 1).

\subsection{Vertical axis wind turbine (VAWT)}

The main rotor shaft of this type of turbines are arranged vertically, hence the name. The major advantage of this arrangement is the turbine does not need to follow the direction of the wind to exhibit high efficiency, which is advantageous in sites with highly variable wind directions. Also advantageous is its ability to be mounted on a building because it is much less steerable. The drivetrain and electrical machine can also be positioned close to the ground with the aid of a direct drive from the rotor arrangement to the ground-based gearbox, enhancing availability for repairs. Energy efficiency over time is still poor, a severe drawback. Key disadvantages also include the relatively low rotational speed with the consequence being increased torque with a proportional increase in cost of the drive train, reduced power coefficient, pulsating mechanical torque, and modeling difficulties for accurate wind flow studies leading to issues of rotors design analyses prior to fabrication [16].

\section{Efficiency of the wind turbine system}

The conservation of mass demands that the measure of air in and out of a turbine must be equivalent. Consequently, Betz's Law defines maximum achievable wind power drawn by a wind turbine as 16/27 (59.3\%) of the aggregate kinetic energy of the air entering the turbine. The best hypothetical power yield of a wind turbine is therefore 16/27 times the kinetic energy of the air entering the turbine effective area (Figure 2).

$$
P=\frac{16}{27} \times \frac{1}{2} \times \rho \times v^{3} \times A=\frac{8}{27} \times \rho \times v^{3} \times A
$$




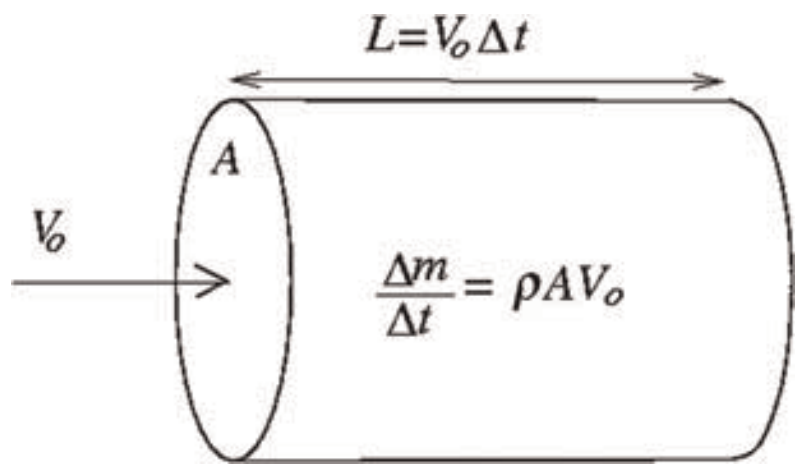

Figure 2.

The air flow through area $A[36]$.

where $\rho$ is the density of air, $A$ is effective area of disk, $v$ is wind velocity, and $P$ is power.

The power accessible from the wind is directly proportional to the cube of the speed of the wind. Meaning if the speed of the wind is doubled then the output power from the turbine is given eight times. Therefore, wind turbine designs have to take this into account by ensuring designs can support higher wind loads than those from which they can generate electricity, in order to prevent them from damage. Wind turbines approach maximum efficiency at wind speeds between 12 and $15 \mathrm{~m} / \mathrm{s}$. Over this wind speed, the power yield of the rotor must be controlled to diminish main thrusts on the rotor blades and in addition the load on the general wind turbine system [17].

As wind energy is free, wind-to-rotor efficiency, losses in the generator and power electronics are the major factors that affect the final cost of wind power generation. To keep parts from corroding, extracted power is fixed above rated operating speed as theoretical power increments at the cube of wind speed, which reduces the efficiency. Turbine efficiency can diminish somewhat after some time because of wear. Examination of 3128 wind turbines 10 years or older in Denmark demonstrated that half of them did not diminish in efficiency, while the other observed a decrease of $1.2 \%$ per year [18]. Vertical turbine efficiency is lower than their horizontal counterparts.

\section{Review of previous works}

A journal article on the feasibility of a simple small wind turbine with variable speed regulation [19] was looked at. The objective of the research was to suggest and evaluate a Very Small Wind Turbine (VSWT) with a Squirrel-Cage Induction Generator (SCIG) that can contend favorably with VSWTs connected to the grid with respect to cost and relative ease. To assess the dynamic response of the system other different wind speeds, two tests were made. The results were gotten from real life scenarios not computer simulations and showed the drop in performance with regards to its nominal value was about $75 \%$, when working at $50 \%$ of the nominal power which is reasonable enough. In summary, the VSWT and its intended control algorithm is feasible for controlling direct-shaft grid-connected VSWTs.

A paper on control of active and reactive power of a wind energy conversion system with variable speed [20] details a design of wind energy conversion system with different speed employing a three-phase SCIG driven by a HAWT. A static VAR compensator is suggested and linked with the SCIG terminals so as to run the system parameters. 
Pitch angle control was utilized in controlling the mechanical power and the system was simulated using SIMULINK software. From the simulation results, the response of the suggested system offers quick recovery faced with different dynamic system disturbances with the controller boosting power thereby improving system efficiency.

A Research by the Czech Technical University studied the need for gearless wind turbines is looked into due to positives such as reliability, and reduction in downtime due to less moving parts. The design simulation was carried out using MATLAB SIMULINK. Results showed the wind turbine had the ability to sustain an electric-power scheme. The system allowed for the independent control of both reactive and active power, suggesting that the gearless design is suitable for turbines with variable speed [21].

In a paper on the transient response of Doubly Fed Induction Generator using an accurate model [22], the transient execution of various models of DFIG considering saturation impact was looked at and a few parameters that influence rotor overcurrent due to voltage sag was simulated. The findings from the paper include the importance of consideration of saturation effect on transients but less so for steady state analysis. Also, the rotor speed of saturated model reaching steady state value quicker than unsaturated model.

A detailed model of fixed speed wind turbine (FSWT) stability studies with stator transient was addressed in a PhD work by [23]. The addition of the stator current transient permit a precise speed divergence forecast. A model for stability of power system analysis like Doubly-Fed Induction Generator (DFIG) wind turbine was also suggested in the same work including the stator flux transient. By doing so, the analysis of Fault Ride-Through (FRT) is done. However, such representation gives rise to difficulties when looking into the implementation of the positive sequence fundamental frequency simulation tools, as a result of small time-step prerequisite and inconsistency with normal power system parts.

A model of DFIG wind turbine was introduced in [24], the stator transient was not considered at normal operation. However, the use of a current controller still demands high simulation resolution.

A basic model of a DFIG wind turbine, compatible with the natural frequency representation was projected by [25]. Both stator and rotor flux dynamics were neglected in the model. This model is comparable to a steady state representation, while the controller of the rotor current is assumed to be instantaneous. Therefore, iteration process which is not favorable in the implementation model is required to solve algebraic loops between the grid model and the generator model.

With the introduction of time lags representing current control delays, algebraic loops can be avoided [26]. Nevertheless, it is assumed that the maximum power tracking (MPT) in this model is directly proportional to the arriving wind speed, although in common practice, the generator speed or the generator output power drives the MPT.

Miller et al. [27] presented another DFIG simplified model. According to this model, the generator is simply modeled as a current source that is controlled; hence the rotor parameters are omitted. This proposed simplified model did not take into account the limiters of rotor current and the FRT schemes are not clearly modeled.

Demonstrations of detailed FSWT models for power system are presented in [28]. In this paper, the generators are modeled thoroughly. They need very small time-step therefore complicating the execution in a standardized fundamental frequency simulator. Ref. [29] proposed a simplified model of an FSWT model.

In a power system network comprising different generation unit, there is bound to be frequency stabilization and control issues. In the work of [31] the frequency responses of the grid power system network and other variables of the grid connected wind during the period of grid dynamics show improved performance as shown in the simulation results. 


\section{Variable speed and fixed speed wind turbines}

The difference between the variable speed and fixed speed wind turbines is whether the rotor is designed to run at different speed or constrained to move at a particular speed. Early wind turbine designs generally operated at constant speed. In this type, the rotor speed does not change regardless of wind speed changes. A converter of power electronic frequency is needed in order to link the variablefrequency output of the wind turbine to the constant electrical system frequency. Power electronics required for different speed wind turbines may be more costly, but they make up for the higher costs by spending more time than fixed turbines working at optimum aerodynamic efficiency [33]. A graph of the performance coefficient versus the tip speed ratio shows this difference clearly. Tip speed ratio is known as the ratio between the angular velocity of the blade tips of a turbine and the wind velocity as shown in Eq. (7). In wind turbine with fixed speed, $\omega$ is constant, corresponding to a specific wind speed. Hence for any other speed from the wind, the turbine efficiency is reduced.

The aim of the wind turbine with variable speed is to always run at optimal efficiency, with tip speed ratio consistency, corresponding to the maximum performance coefficient, by adapting the velocity of the blades to variations of wind speed. Therefore, wind turbines with variable speed designs are ideal for efficient power generation, regardless of the wind speed. Then again, as a result of the fixed speed operation for constant speed turbines, any variations in the speed of the wind are communicated as instabilities in the mechanical torque and then as instabilities in the electrical power grid [17]. This as well as an increased energy capture capability of the variable speed turbine makes the power electronics cost effective [33]. Therefore, wind turbines with variable-speed are more preferable.

\section{Methodology}

This section introduces the model of the wind turbine while analyzing all three blocks. The equations used in the design model for each block are derived and analyzed as well. The wind energy conversion system modeling is reduced into three subsystems which are the aerodynamics block, mechanical block, and the electrical block as shown in the block diagram in Figure 3.

The aerodynamics block is responsible for the extraction of power from the wind in the form of kinetic energy necessary to propel the blades. The mechanical block then converts this kinetic energy into mechanical energy used to drive the generator which in turn is turned into electrical energy by the electrical block.

The modeled wind turbine system is designed and simulated with the MATLAB SIMULINK software. The simulation model diagram showing all three subsystems is shown in Figure 4.

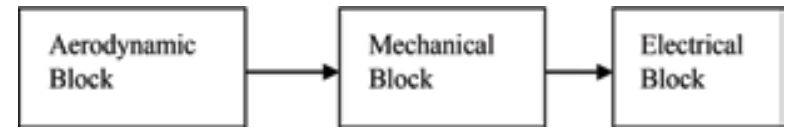

Figure 3.

Block diagram of the conversion system model. 
Modeling and Simulation of a $10 \mathrm{~kW}$ Wind Energy in the Coastal Area of Southern Nigeria... DOI: http://dx.doi.org/10.5772/intechopen.85064

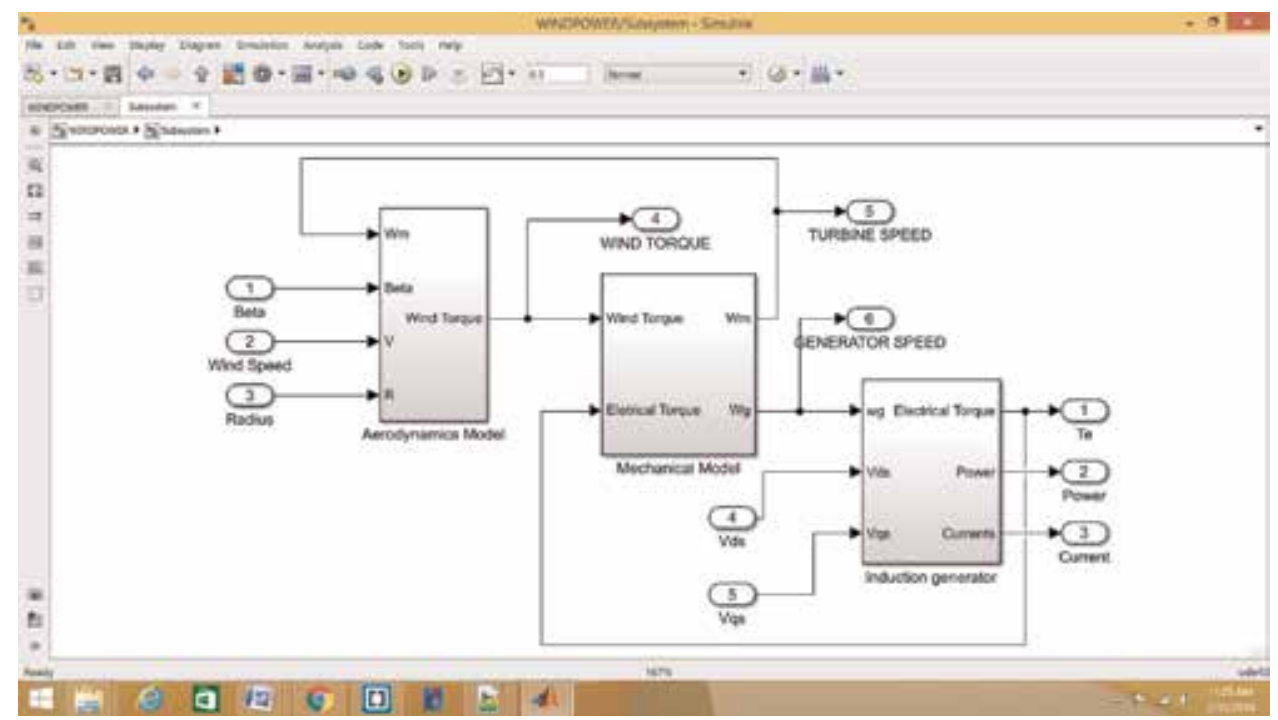

Figure 4.

Simulation schematic diagram of the wind turbine model.

\section{Aerodynamic subsystem modeling}

The wind turbine blades and its interaction with the wind make up the aerodynamic subsystem to be modeled. The aerodynamic modeling block diagram is shown in Figure 5.

The blades of a wind turbine rotate due to kinetic energy from the wind which is defined by the wind speed. An object with mass $(m)$ which moves at velocity $(v)$ has kinetic energy in the air given by [17] as:

$$
E=\frac{1}{2} \times m \times v^{2}
$$

The power contained in the moving blades assuming constant velocity is equal to the differential of this kinetic energy with respect to time as given in (Eq. (3)).

$$
P_{w}=\frac{d E}{d t}=\frac{1}{2} \times m \times v^{2}
$$

where $m$ represents the mass flow rate per second.

When the air crosses the area " $A$ " brushed by blades of the rotor, the power in air can be calculated with (Eq. (4)).

$$
P_{w}=\frac{1}{2} \times v^{3} \times A \times \rho
$$

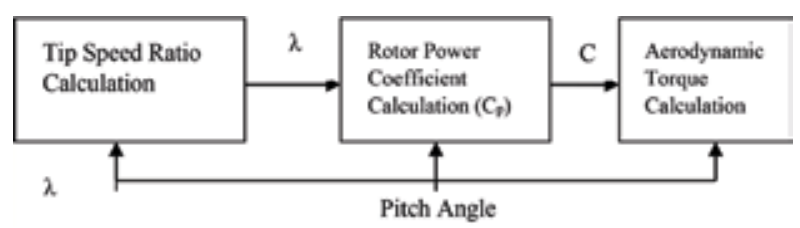

Figure 5 .

Block diagram of the aerodynamic model. 
$\rho=$ the density of air.

Density of air can be conveyed as a component of the turbine's rise above sea level $(H)$ as shown in Equation (5).

$$
\rho=\rho_{0}-1.194 \times 10^{-4} \times H
$$

where $\rho_{0}=1.225 \mathrm{~kg} / \mathrm{m}^{3}$ which is the air density at sea level at temperature $T=298 \mathrm{~K}$. The power extracted from wind is defined as [32],

$$
P_{B L A D E}=C p(\lambda, \beta) \times P_{w}=C p(\lambda, \beta) \times \frac{1}{2} \times v^{3} \times A \times \rho
$$

$C p=0.593$ (Betz law). The rotor power coefficient is a function of both the tip speed ratio " $\lambda$ " and the blade pitch angle " $\beta$ " (in degrees). The blade pitch angle is characterized as the angle between the blade cross-area and the plane of rotation. It alludes to changing the attack angle to best suited angles to adjust the rotation speed of the blades hence adjusting generated power.

Tip speed ratio is defined in Eq. (7),

$$
\lambda=\frac{w_{m} \times R}{v}
$$

$w_{m}$ is angular velocity of the rotor, $R$ blade radius, and " $w_{m}{ }^{*} R$ " is the blade tip speed.

The rotor torque is therefore defined as,

$$
\mathrm{T}_{\mathrm{w}}=\frac{P_{B L A D E}}{w_{m}}=\frac{C p(\lambda, \beta) \times \frac{1}{2} \times v^{3} \times A \times \rho}{w_{m}}
$$

And, $A$, the area covered by the blade

$$
A=\pi \times R^{2}
$$

Substitute Eq. (9) into Eq. (8), giving Eq. (10).

$$
\mathrm{T}_{\mathrm{w}}=\frac{P_{B L A D E}}{w_{m}}=\frac{C p(\lambda, \beta) \times \frac{1}{2} \times v^{3} \times \pi \times R^{2} \times \rho}{w_{m}}
$$

The power coefficient $C p$ can be expressed as shown in Eq. (11).

$$
C p(\lambda, \beta)=c_{1} \times\left(c_{2} \times \frac{1}{y}-c_{3} \times \beta-c_{4} \times \beta^{x}-c_{5}\right) \times e^{\frac{-c_{6}}{y}}
$$

where gamma " $y$ " is given as [32],

$$
\frac{1}{y}=\frac{1}{\lambda+0.08 \beta}-\frac{0.035}{1+\beta^{3}}
$$

where $c 1-c 6$ are the aerodynamic coefficients given as $c 1$ is $0.5176 ; c 2$ is 116 ; $c 3$ is $0.4 ; c 4$ is 5 ; $c 5$ is 21 ; and $c 6$ is 0.0068 .

The simulation schematic of the aerodynamic modeling is shown in Figure 6.

The equations used are (Eq. (7)) for the Lambda, the beta is the pitch angle for the wind turbine blades. The gamma function block uses (Eq. (11)), the power coefficient is modeled using (Eq. (11)) and the wind torque is modeled using (Eq. (10)). 


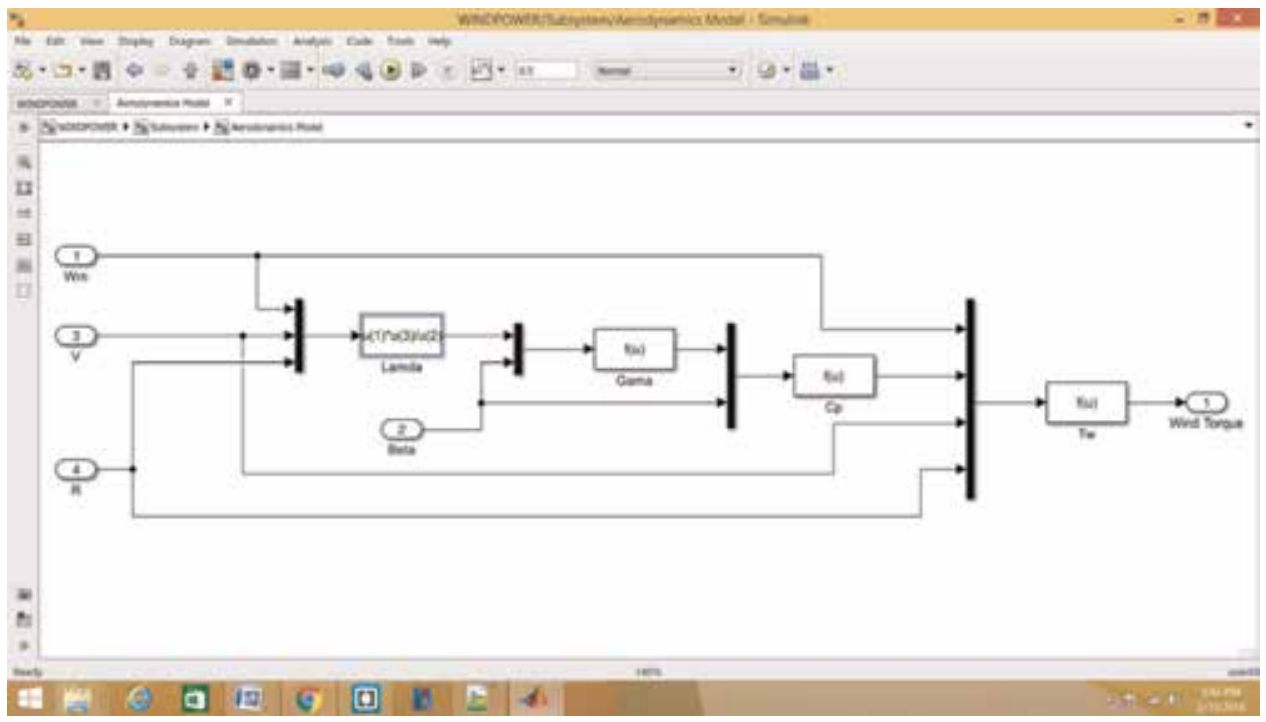

Figure 6.

Simulation schematic diagram of the aerodynamic block.

\section{Mechanical subsystem modeling}

The wind turbine mechanical subsystem is known as the drive train. It comprises of a blade pitching component, a hub with blades, a rotor shaft and a gearbox (Figure 7).

The mechanical model of the wind turbine will be modeled based on two lumped masses assumptions: the gear box mass and the wind wheel mass.

The induction generator equation of motion as given [35] is defined as:

$$
H_{g} \times \frac{d W_{g}}{d t}=T_{e}+\frac{T_{m}}{n}
$$

where $T_{e}$ is Electromagnetic torque, $T_{m}$ is Mechanical torque, $T_{w}$ is wind torque.

Since the wind turbine shaft and generator are linked utilizing a gearbox, the shaft of the turbine is not viewed as stiff. Hence there will be movement in the shaft. The equation of motion of the drive train shaft is computed as

$$
H_{m} \times \frac{d W_{m}}{d t}=T_{w}-T_{m}
$$

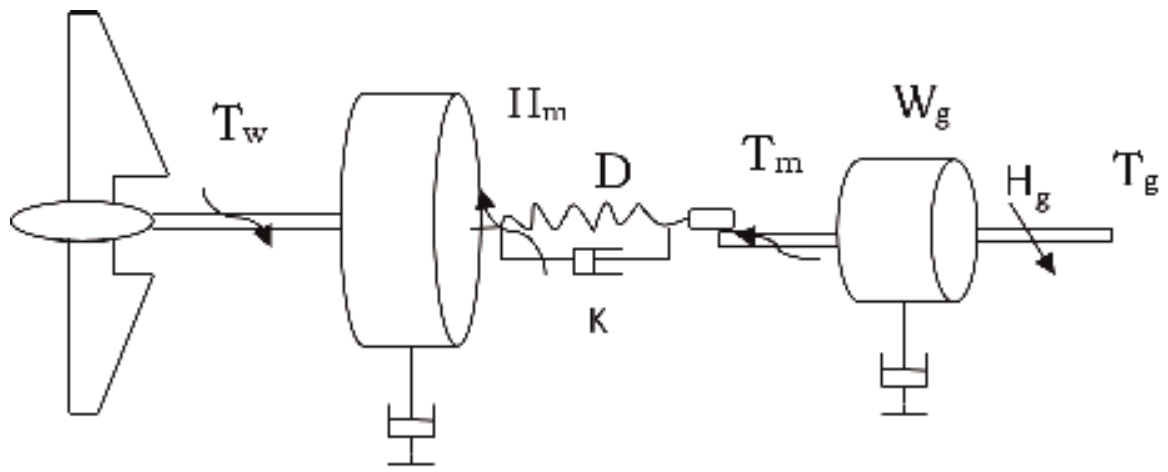

Figure 7.

Mechanical subsystem of a wind turbine. 


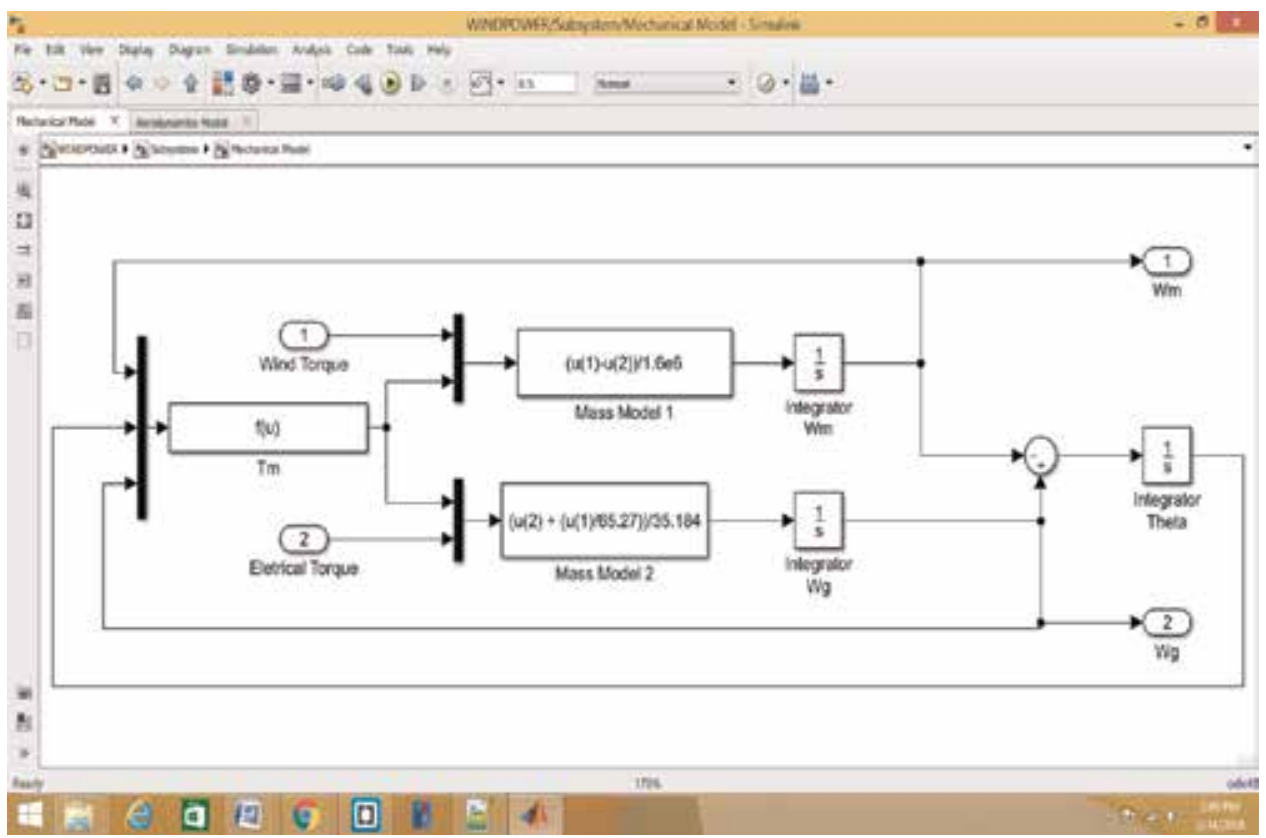

Figure 8.

Simulation schematic diagram of the mechanical model.

$T_{m}$ is given by

$$
\begin{gathered}
T_{m}=K \times \frac{\theta}{n}+D \times \frac{W_{g}-W_{m}}{n} \\
\frac{d \theta}{d t}=W_{g}-W_{m}
\end{gathered}
$$

where, $n$ is the gear ratio; $\theta$ is the angle between the turbine rotor and the generator rotor; $W_{m}$ is the speed of the turbine; $W_{g}$ is the speed of the generator; $H_{m}$ is the turbine inertia constant; $H_{g}$ is the generator inertia constant; $K$ is drive train stiffness; and $D$ is damping constant [35] (Figure 8).

\section{Generator model}

An induction generator (asynchronous generator) is used in this model. This is because of its high reliability and low maintenance compared to synchronous generators. The power captured by the drive train of the turbine is converted to electrical power which takes the form of an alternating current. The induction generator has three-phase stator armature windings (AS, BS, CS) and three-phase rotor windings ( $\mathrm{AR}, \mathrm{BR}, \mathrm{CR})$. The external stationary part is known as the stator and the rotor the internal rotating part of the generator. The rotor is placed on bearings fixed to the stator. At the point when the wind torque exerted on the rotor is enough to drive it beyond synchronous speed, there is electrical energy generated.

When modeling the induction machine the following assumptions are made as described by the mathematical modeling of induction generator for power systems principles. 
Modeling and Simulation of a $10 \mathrm{~kW}$ Wind Energy in the Coastal Area of Southern Nigeria... DOI: http://dx.doi.org/10.5772/intechopen.85064

- When streaming toward the network, the stator currents are positive.

- The real and reactive powers are positive.

- The stator and rotor windings are as far as the mutual effect with the rotor is concerned, are set sinusoidally along the air-gap.

- The stator openings show no considerable differences of the rotor inductances with rotor position.

- The rotor openings show no considerable variations of the stator inductances with rotor position.

- Magnetic hysteresis can be neglected as well as saturation effects.

- The stator and rotor windings are symmetrical.

- Also neglected is the capacitance of all the windings.

In order to simulate the induction generator in SIMULINK; the three-phase supply is converted into a two-phase supply using the help of "Parks Transformation Matrix" where the flux linkage is taking as a staple variable. After conversion, both phases are called $\mathrm{d}$-axis and q-axis. The conversion process is not covered here. When performing power system dynamic studies of induction generator, two models exist;

- A complete model which comprises of electromagnetic transients both in the rotor and the stator circuits, it contains four electromagnetic variables. This is known as the fifth order model.

- The simplified model neglects the stator transients which contains two electromagnetic state variables. This is also known as the third order model.

\subsection{Model including stator transients}

The modeling of the asynchronous generator can be done by finding an equation that relates $V_{d s}, V_{q s}$, the stator direct and quadrature axis voltages, to $I_{d s}, I_{q s}$, the stator direct and quadrature axis currents. The $0 \mathrm{dq}$ reference outline model takes positive currents while rotating at synchronous speed and can be represented using the below equations [34].

- Magnetic fluxes

$$
\begin{gathered}
\varphi_{d s}=X_{s} \times I_{d s}+X_{m} \times I_{q r} \\
\varphi_{q s}=X_{s} \times I_{q s}+X_{m} \times I_{q r} \\
\varphi_{d r}=X_{r} \times I_{d r}+X_{m} \times I_{d s} \\
\varphi_{q r}=X_{r} \times I_{q r}+X_{m} \times I_{q r s}
\end{gathered}
$$

- Voltages

$$
\begin{aligned}
& V_{d s}=-R_{s} \times I_{d s}+w_{s} \times \varphi_{q s}-\frac{d \varphi_{d s}}{d t} \\
& V_{q s}=-R_{s} \times I_{q s}-w_{s} \times \varphi_{d s}-\frac{d \varphi_{d s}}{d t}
\end{aligned}
$$




$$
\begin{aligned}
& V_{d r}=0=-R_{r} \times I_{d r}+w_{s} \times s \times \varphi_{q r}-\frac{d \varphi_{d r}}{d t} \\
& V_{q r}=0=-R_{r} \times I_{q r}+w_{s} \times s \times \varphi_{d r}-\frac{d \varphi_{q r}}{d t}
\end{aligned}
$$

Here, the sub-indexes $(s, r)$ represent the rotor and stator quantities and the subindexes $(d, q)$ represent the $d$ - and $q$-axis in the synchronous rotating reference. The rotor voltages $V_{d r}$ and $V_{q r}$ are equated to zero because current is fed into the stator. The variable $\phi$ represents the magnetic linkage flux, $w_{s}$ represent the synchronous rotor speed and $w_{g}$ represent the generator rotor speed. The slip of the rotor " $s$ " is given as

$$
s=\frac{w_{s}-w_{g}}{w_{s}}
$$

The electrical parameters $R_{s}, X_{s}, X_{m}, R_{r}$ and $X_{r}$ represent the stator resistance and reactance, mutual reactance and rotor resistance and reactance, respectively.

The electrical torque is given as

$$
T_{e}=\varphi_{q r} \times I_{d r}-\varphi_{d r} \times I_{q r}
$$

The power generated by the wind turbine is expressed as

$$
\begin{gathered}
P=P_{\text {active }}+Q_{\text {reactive }} \\
P_{\text {active }}=V_{d s} \times I_{d s}+V_{q s} \times I_{q s} \\
Q_{\text {reactive }}=V_{q s} \times I_{d s}-V_{d s} \times I_{q s}
\end{gathered}
$$

\subsection{Model neglecting stator transients}

Neglecting the stator transients reduces the overall order of the model and increases the size of the system that can be simulated. In this model, the rate of change of stator flux linkage is dismissed. The terms $d \phi_{d s} / d t$ and $d \phi_{q s} / d t$ in Eqs. (21) and (22) will be neglected. Eqs. (17)-(29) are used in the modeling of the induction generator subsystem. The simulation schematic is shown in Figure 9.

The model is designed using attributes of steady-state power of a turbine. There is infinite drive train stiffness and friction factor and turbine inertia are joined to the turbine. Eq. (30) gives the output power of the turbine,

$$
P_{m}=C_{p} \times(\lambda, \beta) \times \frac{\rho A}{2} \times v_{\text {wind }}^{3}
$$

where, $P_{m}$ is mechanical output power $(W)$.

The mechanical power in per unit is expressed in Equation (31).

$$
P_{m \_p u}=k_{p} \times C_{p_{-} p u} \times v_{\text {wind_p } u^{\prime}}^{3}
$$

where $p u$ is per unit.

\section{Case study}

Wind speed attributes are considered stronger in coastal areas and offshore as stated in Section 2 above. This study utilized data from Ogoja community in Cross 
Modeling and Simulation of a $10 \mathrm{~kW}$ Wind Energy in the Coastal Area of Southern Nigeria... DOI: http://dx.doi.org/10.5772/intechopen.85064

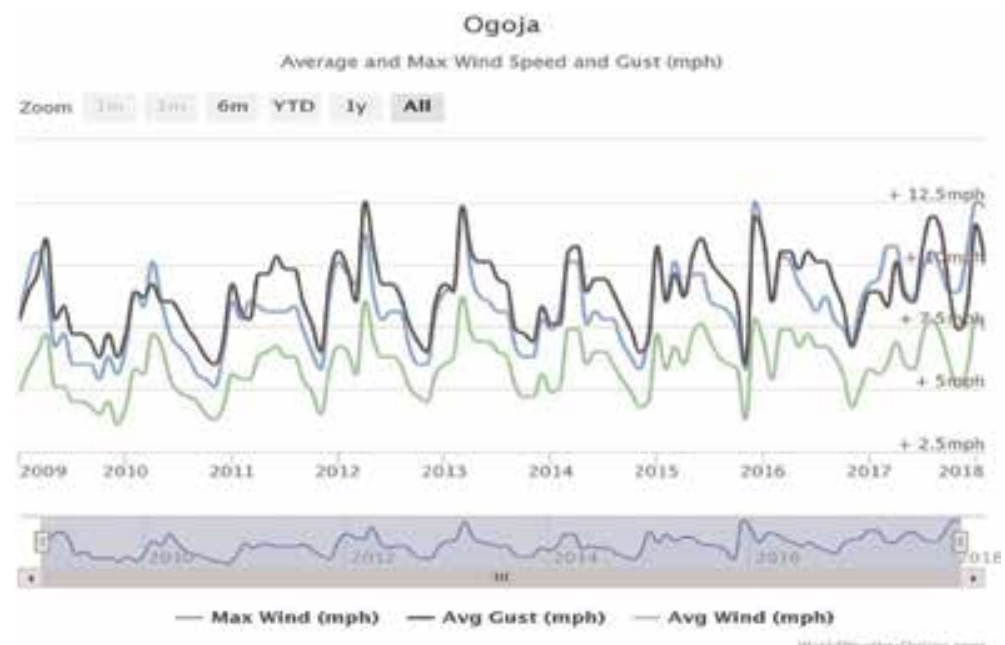

Figure 10.

Average wind speed in Ogoja.

River State (N6.67, E8.48). Monthly data averages on wind speed, temperature and humidity for the last 9 years (January 2009-December 2018) measured at a height of $10 \mathrm{~m}$ was gotten from the World Weather Center and presented in Figure $\mathbf{1 0}$ and Tables 1 and 2.

\section{Simulation result}

At rated wind speed of $3.2 \mathrm{~m} / \mathrm{s}$ (Table 1) the relationship between power and the speed of turbine is shown in Figure 13. The results of the simulation are as

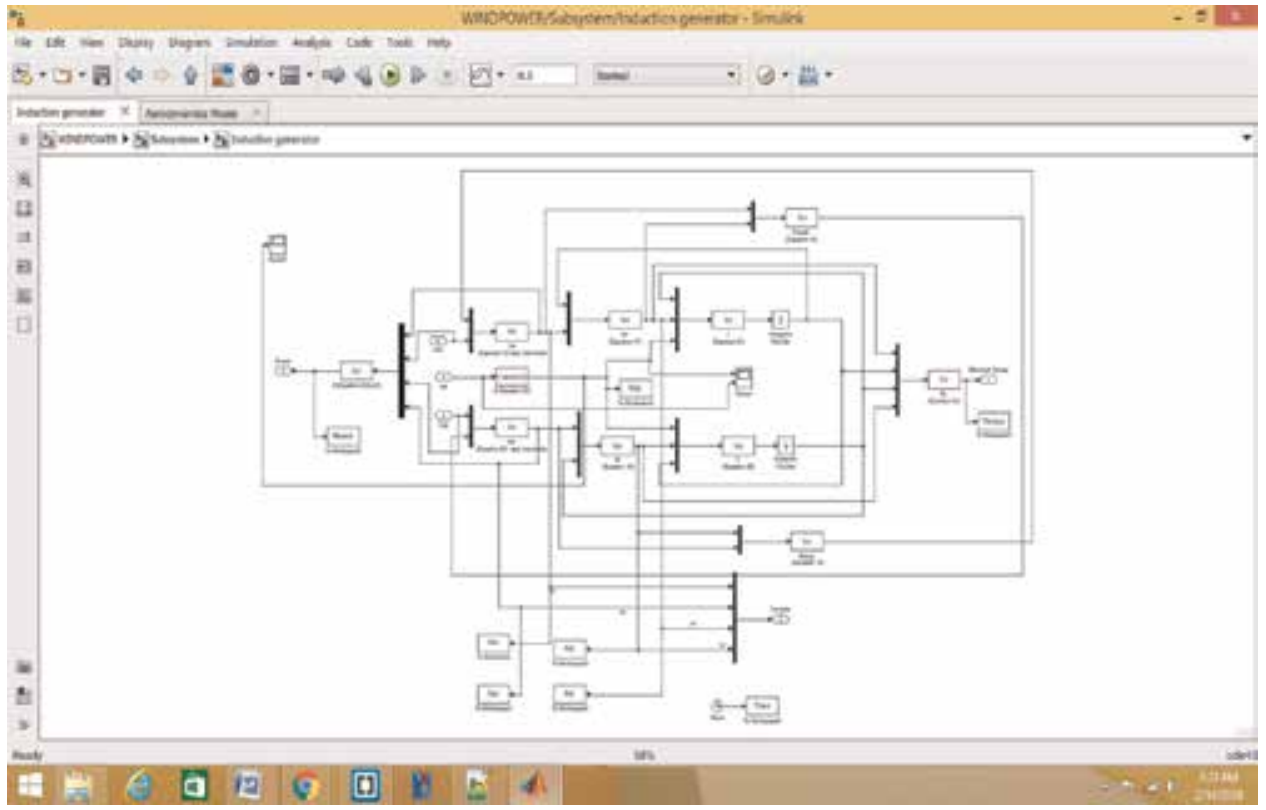

Figure 9.

Simulation schematic diagram of the induction generator subsystem. 

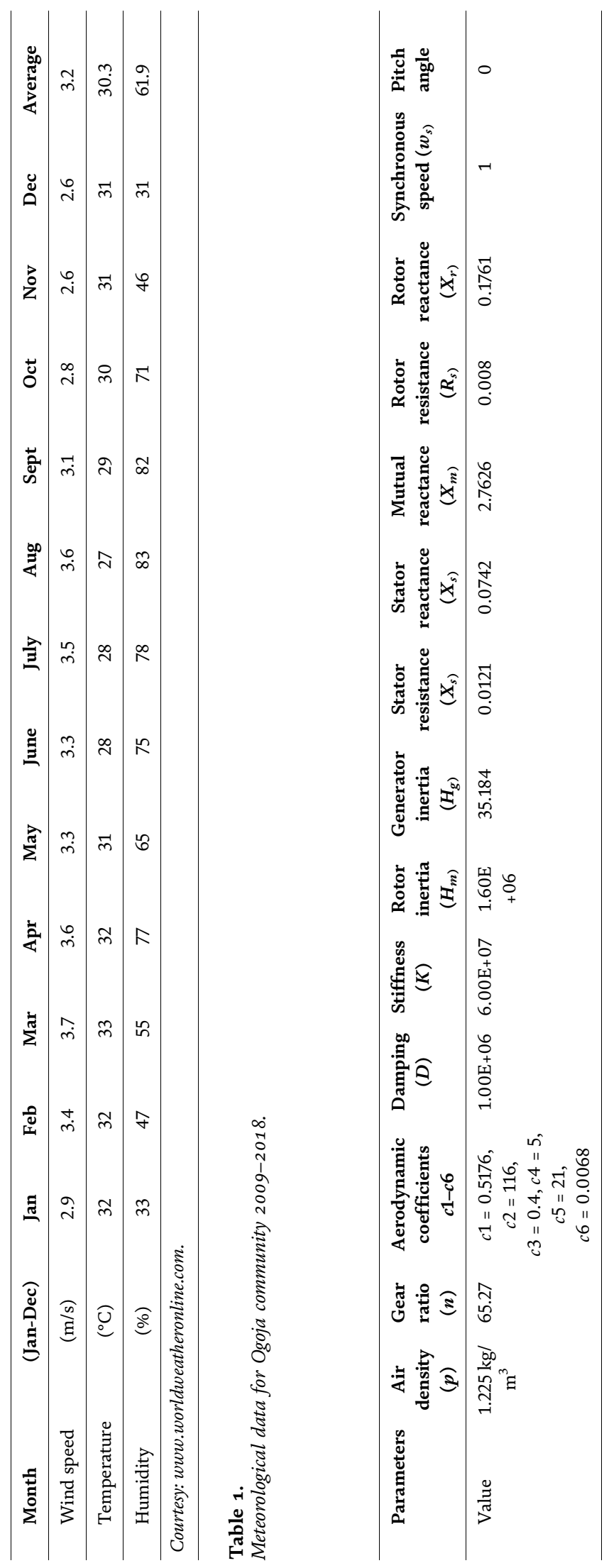

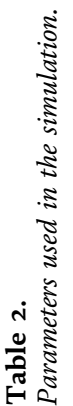


Modeling and Simulation of a $10 \mathrm{~kW}$ Wind Energy in the Coastal Area of Southern Nigeria... DOI: http://dx.doi.org/10.5772/intechopen.85064

shown in the readings captured from the scopes in the simulation model

(Figures 11 and 12).

Figures 13-16 shows different wind torques and the mechanical torque at 1 and $3.2 \mathrm{~m} / \mathrm{s}$ is shown on the scopes. It is seen that an increase in speed of wind also leads to an increase in mechanical torque and in the same direction as the wind torque (Figures 17 and 18).

From the graphs of the turbine speed and the generator speed, the generator moves in the reverse direction of the wind torque shows that the induction machine is used in the generator mode so it tends to negative. The turbine speed starts increasing gradually as the system generates power, it moves in the positive

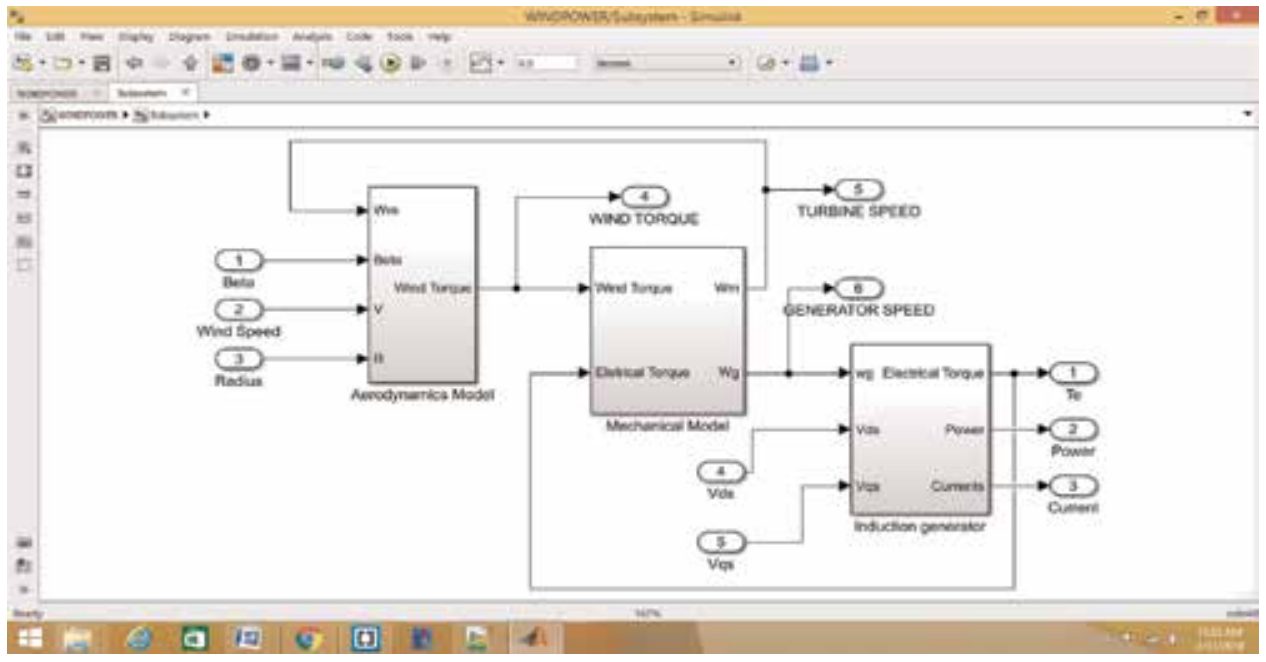

Figure 11.

Simulation schematic diagram of the wind energy conversion system.

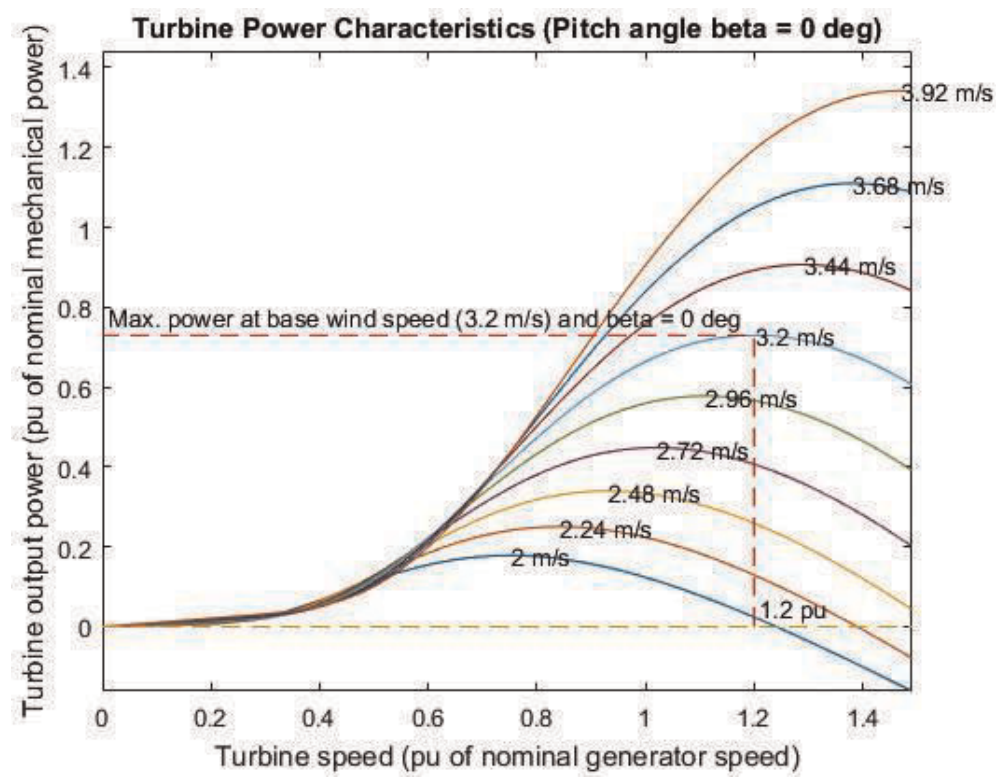

Figure 12.

Relationship between wind speed and output power for the wind turbine. 


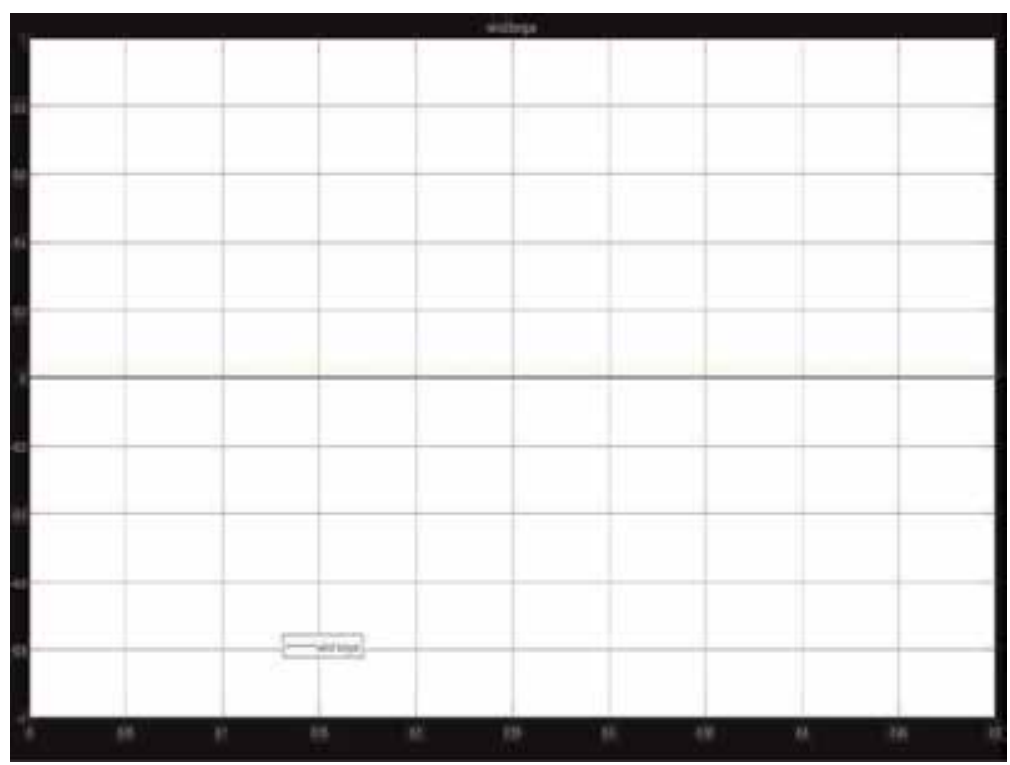

Figure 13.

Wind torque@om/s.

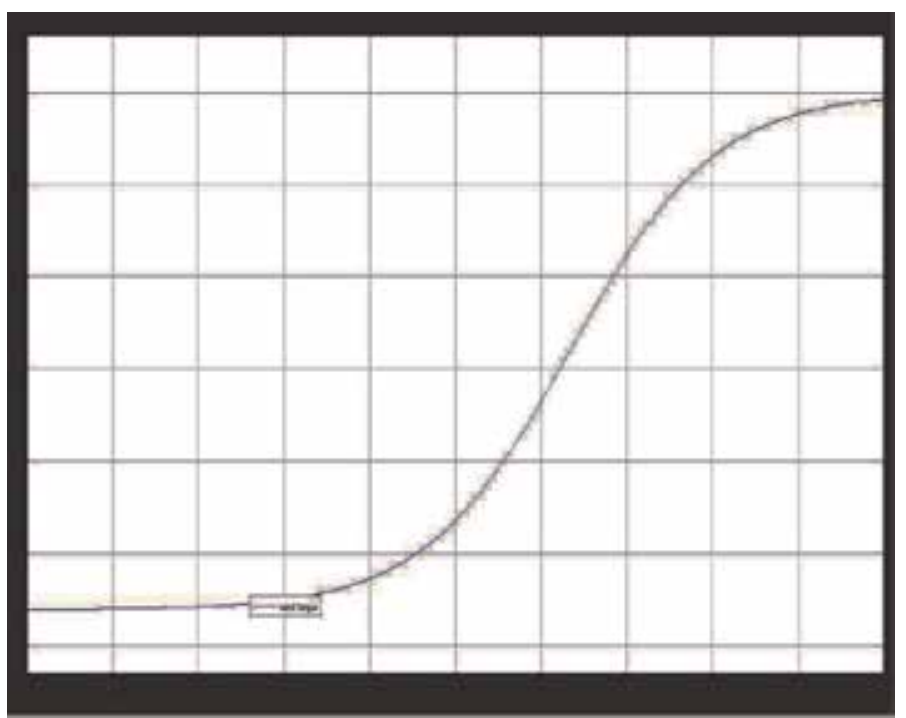

Figure 14.

Wind torque@1 m/s.

direction. The higher the speed, the more power that will be generated. Hence the generator speed is proportional to the turbine speed. The speed from the wind is not sufficient to move the turbine blades until around $0.35 \mathrm{~s}$ when the speed starts progressing gradually, leading to a proportional decrease in generator speed (Figure 19).

The turbine generates fluctuating values of electrical power peaking at about $80 \mathrm{~W}$ at $0.25 \mathrm{~s}$ in the simulation. These results are gotten at rated wind speed of $3.2 \mathrm{~m} / \mathrm{s}$ and zero pitch angle. 
Modeling and Simulation of a $10 \mathrm{~kW}$ Wind Energy in the Coastal Area of Southern Nigeria... DOI: http://dx.doi.org/10.5772/intechopen.85064

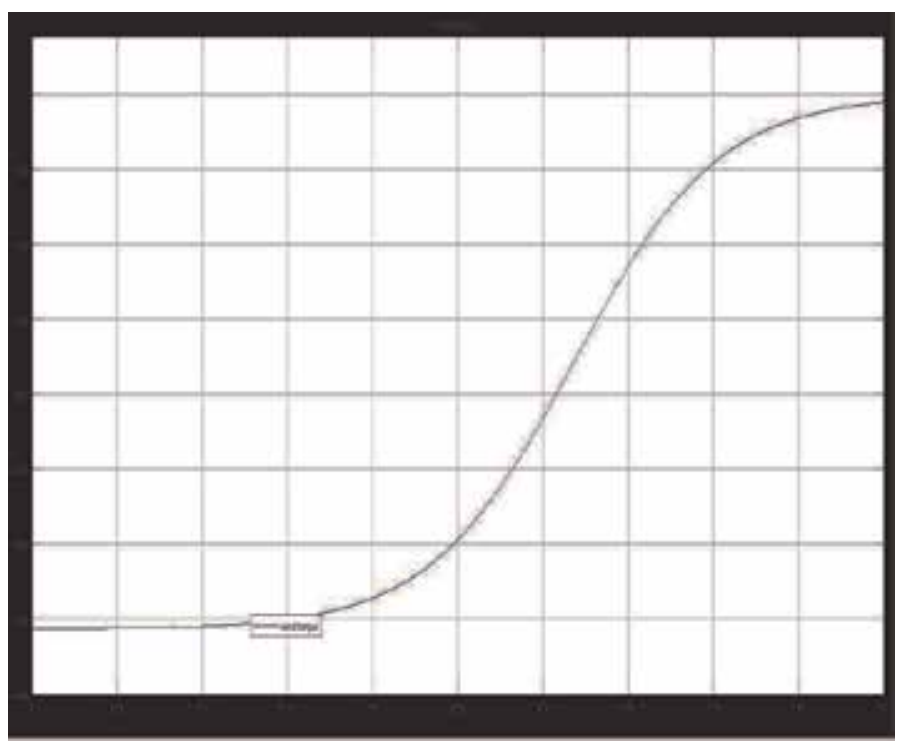

Figure 15.

Wind torque@3.2 m/s.

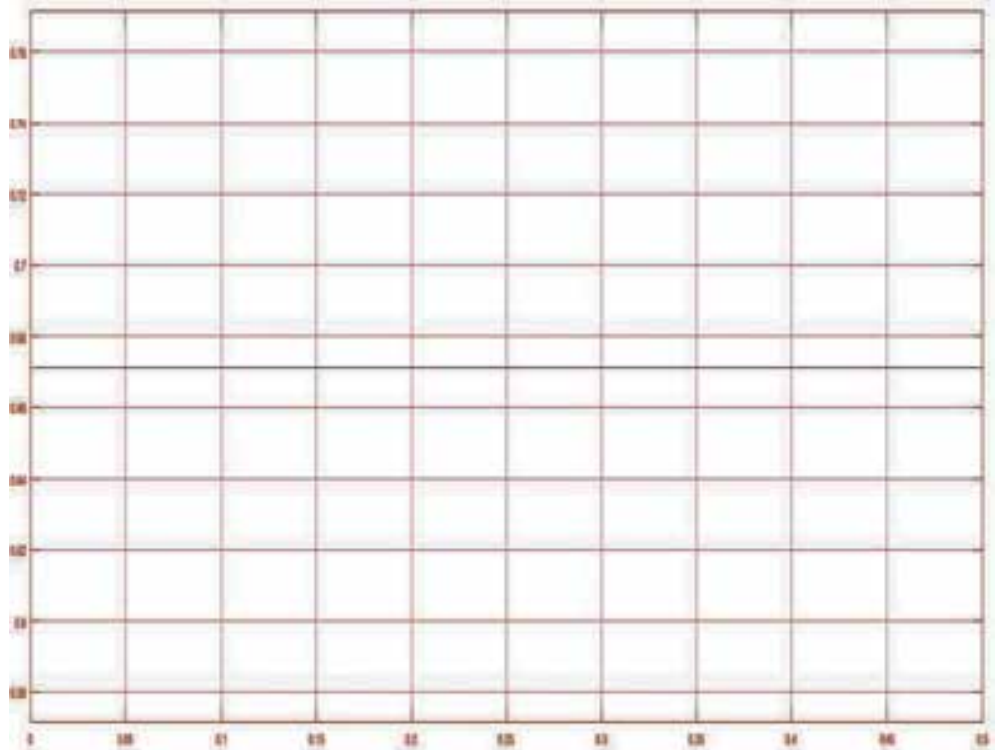

Figure 16.

Mechanicaltorque@ $3.2 \mathrm{~m} / \mathrm{s}$.

\section{Conclusion}

With shortage of energy supply prominent in most developing countries especially in Africa, the need for clean and self-replenishing alternative energy supply cannot be over emphasized. A wind energy conversion system with different wind speed, made up of the blades, drive train and SCIG was modeled for Ogoja community in the southern part of Nigeria as presented. The output power, turbine speed and torque were simulated in MATLAB SIMULINK environment 


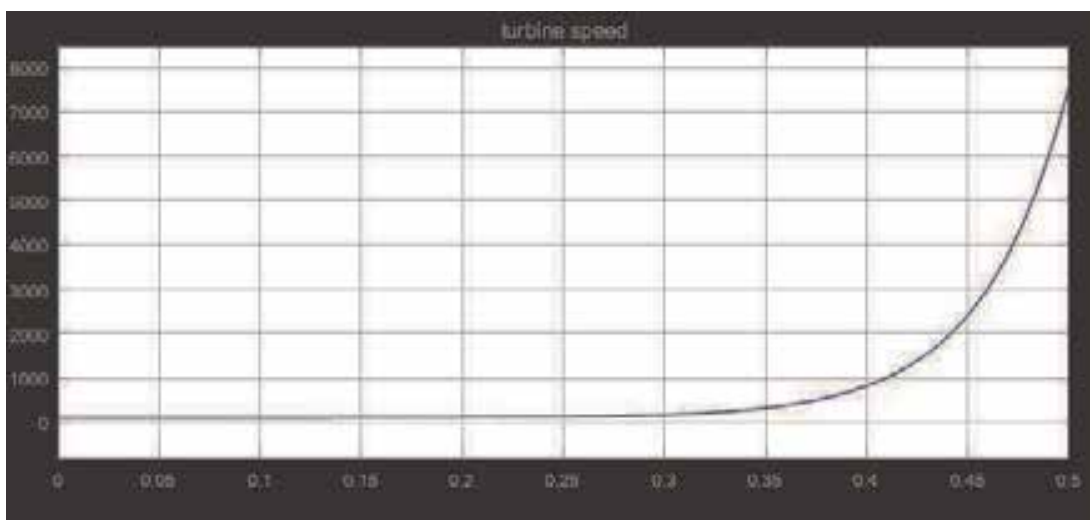

Figure 17.

Turbine speed.

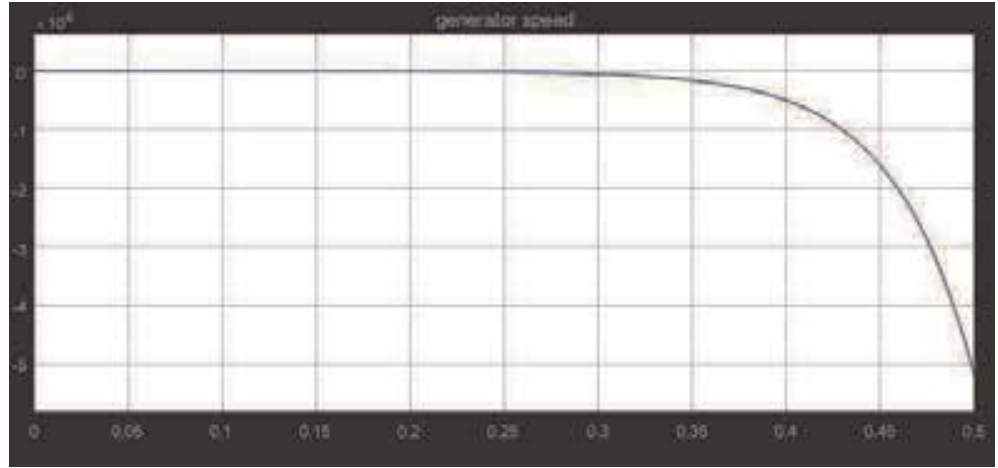

Figure 18.

Generator speed.

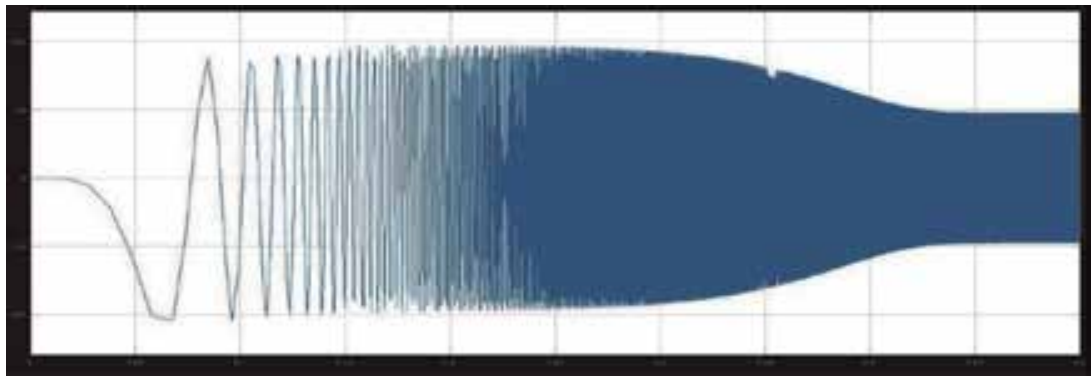

Figure 19.

Electrical power.

successfully. Tests were carried out on the system and showed that turbine speed and wind speed are proportional to power response when simulated. The generated power increases with the turbine speed, making the model useful as wind energy is generated at different wind spend, and this has been able to unveil that even at different wind speed, wind energy potential is available in the coastal area of southern Nigeria. This energy could be used for distributed generation for these communities isolated from the grid. 
Modeling and Simulation of a $10 \mathrm{~kW}$ Wind Energy in the Coastal Area of Southern Nigeria... DOI: http://dx.doi.org/10.5772/intechopen.85064

\section{Author details}

Gabriel Modukpe ${ }^{1 *}$ and Don Diei ${ }^{2}$

1 Rivers State University, Port Harcourt, Nigeria

2 University of Port Harcourt, Port Harcourt, Nigeria

*Address all correspondence to: gabrielmodukpe@gmail.com

\section{IntechOpen}

(C) 2020 The Author(s). Licensee IntechOpen. This chapter is distributed under the terms of the Creative Commons Attribution License (http://creativecommons.org/licenses/ by/3.0), which permits unrestricted use, distribution, and reproduction in any medium, provided the original work is properly cited. (cc) BY 


\section{References}

[1] Chiang EP, Zainal ZA, Narayana A, Seetaramu KN. The potential of wave and offshore wind energy around the coastline of Malaysia that face the South China Sea. In: Proceedings of the International Symposium on Renewable Energy; Kuala Lumpur, Malaysia; September 2003

[2] Kuik V. The lanchester-betzjoukowsky limit. Wind Energy. 2007; 10(3):289-291

[3] KunduruAkhil R, Kalyan D, Arimala P, Sai-purva N. A brief research study, design and analysis on wind turbine. International Journal of Modern Engineering Research (IJMER). 2015;5 (10):6-7

[4] Kenneth OK. Introductory Chapter: Stability control and reliable performance of wind turbine. 2018. p. 4. http://dx.doi.org/10.5772/intechopen. 80237

[5] Idris NA, Lamin HS, Ladan MJ, Yusuf BH. Nigeria's wind energy potentials: The path to a diversified electricity generation-mix. International Journal of Modern Engineering Research. 2012;2(4):2434-2437

[6] Agbetuyi A, Akinbulire T, Abdulkareem A, Awosope A. Wind Energy Potential in Nigeria. 2012. Available from: https://www.researchga te.net/publication/295546689_Wind_ Energy_Potential_in_Nigeria [Accessed: 01 February 2018]

[7] Ajayi O, Fagbenle R, Katende J, Aasa S, Okeniyi J. Wind Profile Characteristics and Turbine Performance Analysis in Kano, International Journal of Energy and Environmental Engineering. NorthWestern, Nigeria. 2013;4(27). Available from: http://www.journal-ijeee.com/ content $/ 4 / 1 / 27$
[8] Okedu KE, Uhunmwangho R, Wopara P. Renewable energy in Nigeria: The challenges and opportunities in mountainous and riverine region. International Journal of Renewable Energy Research, IJRER. 2015;5(1):222-229

[9] Modukpe G, Ndubisi SN. Design of a hybrid/solar gas turbine power system for beta glass delta plant. CiiT International Journal of Automation and Autonomous System. January 2019;11(1)

[10] Lehmeyer International Consultants, Report on Nigeria. 2005

[11] Obiukwu O. Nigeria gets largest wind power plant in West Africa. Article on This-day Newspaper. 2015 [Accessed: 11 January 2019]

[12] Wyatt A. Electric Power: Challenges and Choices. Toronto: Book Press Ltd; 1986. ISBN 0-920650-00-7

[13] Dafrose C, Bayero M. Horizontal and vertical axis wind turbines. 2011. https://colortheearthturquoise.files. wordpress.com/2012/03/horizontaland-vertical-axis-wind-turbines-1.pdf

[14] Lund M. Dansk

FirmaSætterPrisbelønnet Selvhejsende; 2016 [Accessed: 02 November 2017]

[15] Burton T, Jenkins N, Sharpe D, Bossanyi E. Wind Energy Handbook. Chichester West Sussex, England: John Wiley and Sons; 2001. p. 65. ISBN 0471489972

[16] Piggott H. Wind Speed in the CityReality Versus the DTI Database. 2007. Available from: Scoraigwind.com [Accessed: 06 November 2017]

[17] Ackermann T, Söder L. Wind energy technology and current status: A review. Renewable and Sustainable Energy Reviews. 2000;4:315-374 
[18] Sanne W. 11 Years of Wind Data Shows Surprising Production Decrease (in Danish) Ingenioren. 2013 [Accessed: 02 July 2017]

[19] Cuesta AB, Gomez-Gil FJ, Fraile JVM, Rodríguez JA, Calvo JRV, Peláez J. Feasibility of a simple small wind turbine with variable-speed regulation made of commercial components. Energies. 2013;6:3373-3391. DOI: $10.3390 /$ en6073373

[20] Baloch MH, Wattoo AW, Kumar D, Kaloi GS, Memon AA, Tahir S. Active and reactive power control of a variable speed wind energy conversion system based on cage generator. International Journal of Advanced Computer Science and Application (IJACSA). 2017;8(9)

[21] Fandi G, Igbinovia F, Ahmad I, Svec J. Modelling and simulation of a gearless variable speed wind turbine system with PMSG. In: 2017 IEEE PES-IAS Power Africa Conference; Jan. 2017 [Accessed: 10 November 2017]

[22] Alireza A, Saeed L, Vahid M. Transient Response of Doubly Fed Injection Generator using an Accurate Model. 2009 [Accessed: 21 August 2017]

[23] Akhmatov V. Analysis of dynamic behavior of electric power system with large amount of wind power [Ph.D. dissertation]. Technical University of Denmark; 2003

[24] Erlich I, Kretschmann J, Fortmann J, Mueller-Engelhardt S, Wrede $\mathrm{H}$. Modeling of wind turbines based on doubly fed induction generators for power system stability studies. IEEE Transactions on Power Systems. 2007; 22(3):909-919

[25] Ledesma P, Usaola J. Doubly fed induction generator model for transient stability analysis. IEEE Transactions on Energy Conversion. 2005;20(2):388-397
[26] Lei Y, Mullane A, Lightbody G. Modeling of the wind turbine with a doubly fed induction generator for grid integration studies. IEEE Transactions on Energy Conversion. 2006;21(1): 257-264

[27] Miller N, Sanchez-Gasca J, Price W, Delmerico R. Dynamic modeling of GE 1.5 and 3.6 MW wind turbine generators for stability simulations. In: Proceeding of Power Engineering Society General Meeting. Vol. 3. 2003. pp. 1977-1983

[28] Achilles S, P'oller M. Direct drive synchronous machine models for stability assessment of wind farms. In: Proceeding of 4th International Workshop on Large-scale Integration of Wind Power and Transmission Networks for Offshore Wind Farms; Billund, Denmark; 2003

[29] Clark K, Miller N, Sanchez-Gasc J. Modeling of GE wind turbine generators for grid studies. Technical Report. General Electric International, Inc.; 2008

[30] Eltamaly AM. Introduction to Wind Energy System. King Saud University. pp. 33

[31] Okedu EK. A variable speed wind turbine flywheel based coordinated control system for enhancing grid frequency dynamics. International Journal of Smart Grid. 2018;2:2

[32] Jangamshetti S, Naik R. Dynamic Simulation Model of Wind Energy Conversion System. WWEA; 2006. Available from: www.wwec2006.com [Accessed: December 2017]

[33] Balas M, Fingersh L, Johnson K, Pao L. Control of variable speed wind turbines: Standard and adaptive techniques for maximizing energy capture. IEEE Control Systems Magazine. 2006. Available from: www3. imperial.ac.uk/library 
[34] Martins M, Perdana A, Ledesma P, Agneholm E, Carlson O. Validation of fixed speed wind turbine dynamic models with measured data. Renewable Energy. 2007;32:1301-1316. Available from: www.sciencedirect.com [Accessed: 11 December 2017]

[35] Zbibniew L. Wind Turbine Operations in Power System. Berlin: Springer-Verlag; 2003

[36] Hansen MOL. Chapter 9. Aerodynamics and design of horizontalaxis wind turbine. In: Wind Energy Engineering, a Handbook for Onshore and Offshore Wind Turbine. United Kingdom: Academic Press, 2017. p. 162 


\title{
Harmonic Resonance Analysis for Wind Integrated Power System and Optimized Filter Design
}

\author{
Jignesh Pravinbhai Patel and Satish Kantilal Joshi
}

\begin{abstract}
As the contribution of renewable energy sources is increasing year over year, the effect of harmonics on power system becomes important, and it requires special attention. In conventional power sources, the harmonics is not generated at the source side; only load side is contributing in the harmonics. But renewable energy sources, particularly wind and solar, are based on power electronic devices, so it generates harmonics. This harmonics may have an adverse effect on the system. Harmonic resonance is one of the phenomena, due to which the harmonics are amplified and give rise to several trivial issues. Various methods are used to control the harmonics in the system. Harmonic filter is one of the simple ways to absorb the harmonics generated at load and generation side. Various filter designs have been found in literature as well as in the field. The filters are classified according to their design, construction and operation method. There are two main categories, active filters and passive filters. The passive filters are widely used due to its simplicity and lesser cost. However, to achieve the better performance, it is also used with active filters, and this combination is known as hybrid filter. The response of filters is modified as per the system requirement using various techniques. In this work, the impedance characteristics of various filters are discussed and analysed. Also, how the control structure of power electronic devices affects or modifies the output impedance of converter is also discussed.
\end{abstract}

Keywords: voltage source converter (VSC), pulse width modulation (PWM), grid-connected converter, filter, harmonic resonance, doubly fed induction generator (DFIG), Nyquist criteria, damped filter

\section{Introduction}

Voltage source converter (VSC) is widely used in industrial, commercial and renewable power generation applications. Out of many possible configurations, the three-phase and three-wire is widely used. During the last decade, the penetration of renewable energy sources has increased around the world. This is due to the increased concern worldwide about the carbon emission $[1,2]$. The utilisation of energy from uncertain and variable sources has become possible with the use of these converters only.

For the purpose of this study, the analysis of VSCs is carried out assuming the ideal operating conditions of grid. But VSCs are never operating under such 
conditions in practical. In a relatively weak system, VSCs are subject to various power quality disturbances, such as unbalance voltage, voltage swell and swag, notches, etc. [3]. The occurrence of such disturbances causes various problems like ripple in torque of generator and motor, increased losses, abnormal tripping of protective devices, malfunction of sophisticated control system, reduction in the expected lifetime of equipment, etc. [4].

There are two types of harmonics generated by VSCs. One is characteristics harmonics, which are related with the switching operations of the IGBTs inside the VSC. And second is non-characteristics harmonics. The voltage ripple on DC side of VSC generates harmonics on its AC side current. According to [5], the noncharacteristics harmonics are generated by the unbalanced voltage in the AC side. However the quantification of magnitude of such harmonics is not simple and cannot be done with deterministic method. The non-characteristics harmonics are considered as the steady-state low-frequency components which would not appear if the grid voltage is balanced. The unbalance grid voltage has fundamental frequency negative sequence component and third-order positive sequence component.

Though it is possible to eliminate zero sequence third harmonic component using transformer of proper vector group, the non-characteristics third-order positive sequence harmonics cannot eliminate transformers with delta-connected winding.

In [6], the author has proposed DC voltage control to eliminate DC oscillating voltage when AC side is unbalanced. To achieve this, VSC has to operate with constant AC power control. However, the effect of control on AC current is not discussed. It is important to analyse the distorted and unbalanced AC side current, when such control is implemented. In this case, the currents of AC side of converter contain non-characteristics low-frequency component such as fundamental negative sequence and third harmonic components of positive and negative sequence.

\section{Modelling of the voltage source converter}

The total DC current $I_{d c}$ is flowing to the DC side of VSC. $V_{d c}$ is the instantaneous voltage across the capacitor, and id is the instantaneous DC current. The quality factor of DC capacitor is assumed to be high, so the series resistance is neglected. The instantaneous power is supplied by the renewable energy source, i.e. wind turbine. The instantaneous current $I_{s}$ is supplied by the external source. It is equal to zero when VSC operates as a reactive power compensator.

The block diagram of VSC control structure is depicted in Figure 1. The control system consists of (i) voltage control, (ii) a phase lock loop (PLL), (iii) current reference calculation block and (iv) current control. The real power reference is

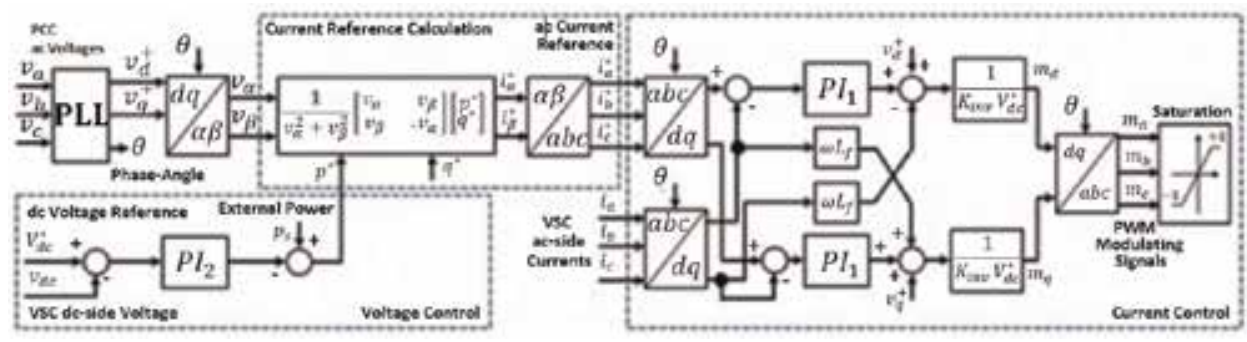

Figure 1.

Control structure of grid-connected wind converters. 
calculated by the PI controller, which considers the DC voltage and desired active power through the VSC to the grid. The instantaneous reactive power is calculated by the separate loop, which may consider either the desired power factor or the reference voltage. The dq frame is synchronised with the positive sequence fundamental voltage of the grid at PCC with the help of PLL. It converts three phase voltages $V_{a}, V_{b}$ and $V_{c}$ into $V_{d^{+}}$and $V_{q^{+}}$, which are converted in the alpha-beta reference frame voltages $V_{\alpha}$ and $V_{\beta}$. Then current reference is obtained by the $\alpha$ - $\beta$ to $a b c$ transformation. These reference currents are compared with actual current, and modulating signals $m_{d}$ and $m_{q}$ are generated. These signals are finally transformed into $m_{a}, m_{\mathrm{b}}$ and $m_{c}$ to generate pulse width modulated (PWM) signal.

Both switching frequency and grid voltage distortion can cause poor power quality. A filter design is a subject that requires trade-off between filter performance and the control bandwidth. Filters are required to meet power quality standard, avoid parallel resonance and improve power quality.

Inverter for grid interfacing will need to incorporate interface filters to attenuate the injection of current harmonics.

\section{Impedance-based stability analysis}

This method was proposed in [7]. The system impedance is partitioned into source and grid impedance. The source impedance is either represented by Thevenin's equivalent circuit or Norton equivalent circuit (Figures 2 and 3). Thevenin's equivalent circuit consists of ideal voltage source in series with the series impedance $\left(Z_{s}\right)$, whereas the load impedance is modelled by series impedance $\left(Z_{l}\right)$. Since the converter circuit is non-linear, it is represented by the small signal circuit. This linear representation of circuit is valid only for the small perturbation of signal. With this assumption, the current (I) flowing from source to load is given by

$$
I(s)=\frac{V_{s}(s)}{Z_{l}(s)+Z_{s}(s)}
$$

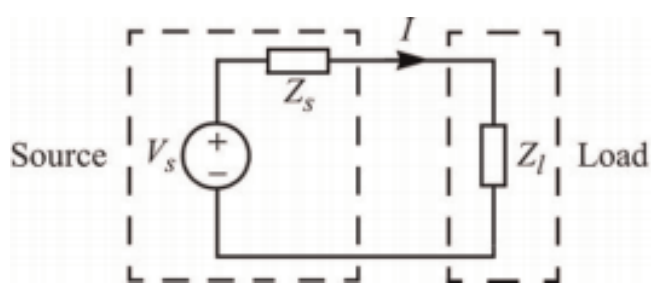

Figure 2.

Thevenin's equivalent circuit.

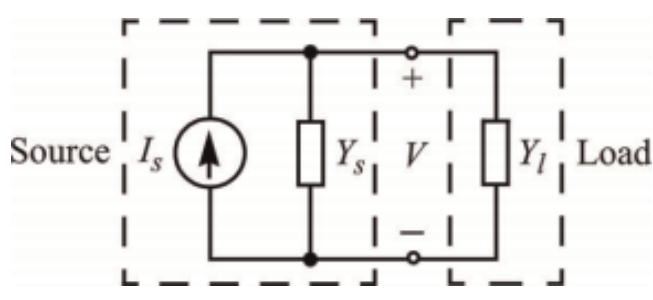

Figure 3.

Norton's equivalent circuit. 
Which is rearranged as,

$$
I(s)=\frac{V_{s}(s)}{Z_{l}(s)} \cdot \frac{1}{1+Z_{s}(s) / Z_{l}(s)}
$$

System stability analysis is based on the assumption that the source voltage and load impedance remain stable. So, $V(s)$ and $1 / Z(s)$ are stable. So, the stability depends on the extreme right-hand side of Eq. (2). It is given by

$$
H(s)=\frac{1}{1+Z_{s}(s) / Z_{l}(s)}
$$

The close observation of Eq. (3) reveals the important characteristics. It is a transfer function with unity gain and feedback equal to $Z_{s}(s) / Z_{l}(s)$. According to the linear control theory, the $\mathrm{H}(\mathrm{s})$ is stable, if and if only, when ration $Z_{s}(s) / Z_{l}(s)$ meets the requirement of Nyquist stability criterion [7].

In the above analysis for stability, it is assumed that the source is ideal voltage source and it remains stable under unloaded condition. However, the gridconnected inverters are usually current controlled. Hence above analysis is not much useful. So, the source should be represented as current source. To arrive at the equivalent current source, the same small signal voltage source is modified. The voltage across load is given by

$$
V(s)=\frac{I_{s}(s)}{Y_{l}(s)+Y_{S}(s)}
$$

by rearranging

$$
V(s)=\frac{I_{s}(s)}{Y_{l}(s)} \frac{I_{s}(s)}{1+\frac{Y_{S}(s)}{Y_{l}(s)}}
$$

Similar to the above analysis, the current source is assumed to be stable under unloaded condition. The load is stable when connected to ideal current source. Under this condition, $I(s)$ and $1 / Y_{l}(s)$ are stable. Under this condition, stability of $V(s)$ depends on stability of second term of Eq. (5). This again resembles the closedloop transfer function with negative feedback. The gain is unity and the feedback factor is $Y_{s}(s) / Y_{l}(s)$. Therefore the system is stable, if, along with above conditions, it meets the Nyquist criterion. In Eq. (5) admittances are used instead of impedance, though the analysis still can be carried out in terms of the impedances. In this case, Eq. (5) becomes

$$
V(s)=I_{s}(s) \cdot Z_{l}(s) \frac{1}{1+\frac{Z_{l}(s)}{Z_{s}(s)}}
$$

It is important to note the requirement of stability. In the voltage source model, the output impedance of source should be as low as possible (ideally zero); whereas in the current source model, the output impedance should be as high as possible (ideally infinite).

\section{Grid-connected inverters}

The modelling of impedance of grid-connected VSC has very important use in analysis of stability and resonance phenomena when converter is integrated into the 
grid [5]. The grid-connected converter used in renewable sources is modelled as a current source in parallel with an impedance, i.e. Norton's equivalent circuit [6]. The stability of grid-connected inverter can be determined by Nyquist criterion [8]. The control structure of most of the VSCs is developed in the rotating dq reference frame [7]. The phase lock loop is used to synchronise converter with grid [9]. The use of complex multiple-loop control structure introduces non-linearities. These are generally overlooked in the simplified low-order modelling [10]. On the flip side, the detailed model introduces complexity and cross-couplings between various terms, which makes the determination of output impedance cumbersome. The trade-off way suggested in some literatures is to linearise the model by small signal analysis technique.

The impedance of converter-interconnected generator is affected by various factors such as control parameters, PLL, switching delays and converter harmonic filters. The converter is basically controlled by output current signal. In Figure 4, $\boldsymbol{i}_{\boldsymbol{1}}$ is the converter current and $i_{2}$ is the grid current. The converter is controlled either by $\boldsymbol{i}_{1}$ or $\boldsymbol{i}_{2}$. If the grid current is the control variable, then the current control loop is

$$
Y_{o}(s)=G_{P I}(s) G_{D}(s) Y_{21}(s)
$$

where $\mathrm{Y}_{21}$ is the forward trans-admittance of the filter, $G_{P I}(s)$ is the proportional-integral-type current controller and $G_{D}(s)$ is the switching delay. Here, the converter output voltage is considered as pure sinusoidal; if there is a noise in the voltage, then it needs to be considered as a disturbance signal. If, the converter is controlled by taking converter current $\boldsymbol{i}_{1}$, then $\mathrm{Y}_{21}$ is replaced by output conductance $Y_{11}$. The forward transconductance $Y_{21}$ is given by

$$
Y_{21}(s)=\frac{Z_{3 f}}{Z_{1 f} Z_{2}+Z_{1 f} Z_{3 f}+Z_{2} Z_{3 f}}
$$

The simple transfer function of current controller is given by

$$
G_{P I}(s)=k_{p}+\frac{k_{i}}{s}
$$

Higher-order controller also can be used for current control, but here simple $P \boldsymbol{I}$ controller is considered for the sake of simplicity. The delay, estimated by Pade's approximation technique, is given by

$$
G_{D}(s)=\frac{1}{1+1.5 T_{d} s}
$$

The frequency response of output impedance is plotted here with the line impedance. The phase at the intersection of two curves gives the phase margin (PM).

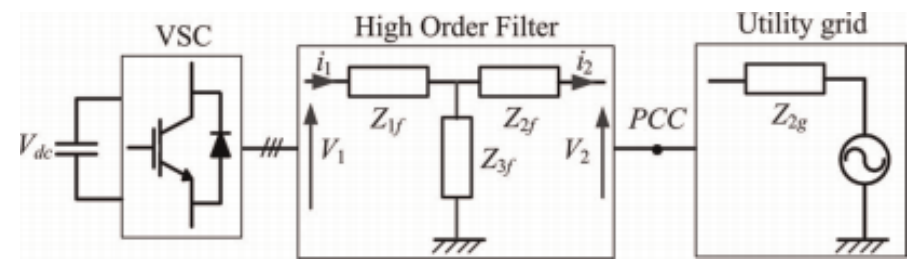

Figure 4.

Converter output circuit (Source: [11]). 


\section{Phase lock loop}

$$
G_{P L L}(s)=f(s, w, k)
$$

The phase lock loop is required for PI-based controller. So, the structure of PLL should be thoroughly assessed for stability. Proper structure of PLL and control scheme helps to mitigate adverse impact on the system. The purpose of PLL is to synchronise converter with grid. Harmonics in the grid may penetrate to the converter through the PI controller. If control parameters are not properly chosen, this may produce harmonics through circular effect. Figure 5 shows the block diagram of PLL. The detail block diagram is given in Figure 6.

Figure 6 is the traditional second-order generalised integrator-quadrature signal generator (SOGI-QSG) PLL. It can filter out higher-order harmonics, where $\boldsymbol{u}_{\boldsymbol{i}}$ is the input signal; $\boldsymbol{u}_{\boldsymbol{i}}{ }^{\prime}$ and $\boldsymbol{q} \boldsymbol{u}_{\boldsymbol{i}}^{\prime}$ are two output signals, which are in quadrature; $\boldsymbol{k}$ is the damping coefficient; and $\omega^{\prime}$ is the output angular frequency of PLL. Eqs. (14) and (15) show the transfer function of PLL.

$$
\begin{gathered}
G_{1}(s)=\frac{u_{i}^{\prime}}{u_{i}} \\
G_{1}(s)=\frac{u_{i}^{\prime}}{u_{i}}=\left[\left(u_{i}-u_{i}^{\prime}\right) k-u_{i}^{\prime} \omega^{\prime} / s\right] * \omega^{\prime} / s=u_{i}^{\prime}
\end{gathered}
$$

Simplifying above equation gives

$$
G_{1}(s)=\frac{u_{i}^{\prime}}{u_{i}}=\frac{k \omega^{\prime} s}{s^{2}+k \omega^{\prime} s+\omega^{2}}
$$

Similarly, for quadrature output transfer function is

$$
H_{1}(s)=\frac{q u_{i}^{\prime}}{u_{i}}=\frac{k \omega^{\prime 2}}{s^{2}+k \omega^{\prime} s+\omega^{\prime 2}}
$$

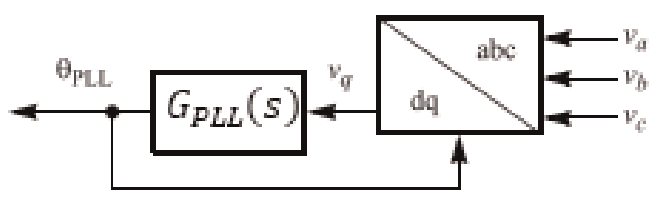

Figure 5 .

Phase lock loop.

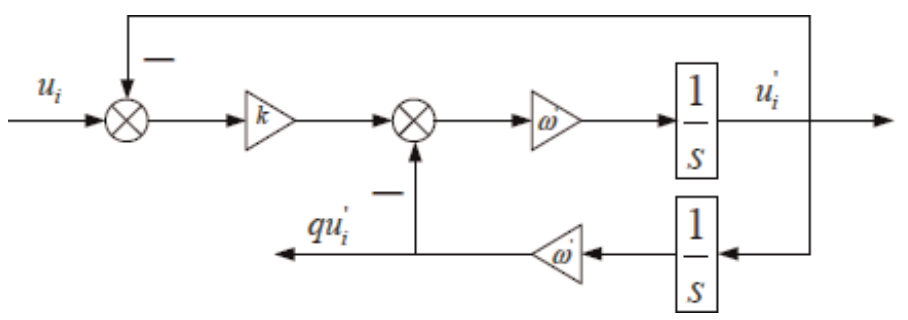

Figure 6.

Block diagram of simple phase lock loop. 


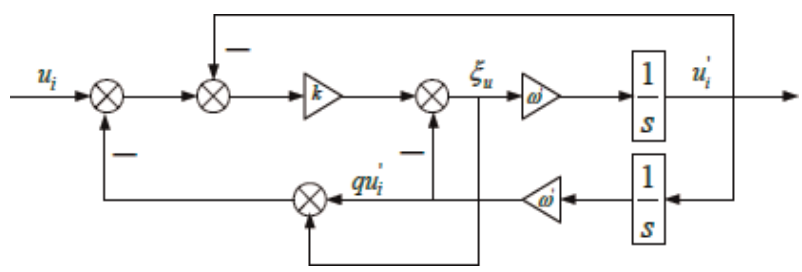

Figure 7.

Block diagram of modified phase lock loop.
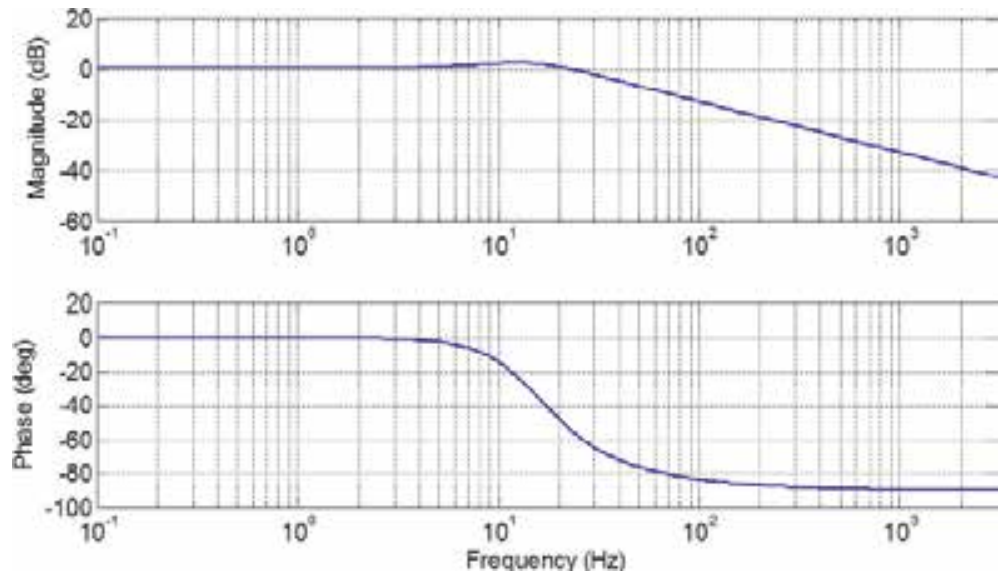

Figure 8.

Frequency response of simple phase lock loop.

In traditional SOGI-QSG PLL, the DC component in the input signal is not suppressed by the PLL. To overcome this problem, a minor modification is made in above PLL. The modified PLL is shown in Figure 7.

Transfer functions of modified PLL are given in Eqs. (16)-(18). This structure reduces the tracking error of PLL.

$$
\begin{gathered}
F_{2}(s)=\frac{\xi_{u}}{u_{i}}=\frac{k s^{2}}{s^{2}+k \omega^{\prime} s+\omega^{\prime 2}} \\
G_{2}(s)=\frac{u_{i}^{\prime}}{u_{i}}=\frac{k \omega^{\prime} s}{(k+1) s^{2}+k \omega^{\prime} s+(k+1) \omega^{\prime 2}} \\
H_{2}(s)=\frac{q u_{i}^{\prime}}{u_{i}}=\frac{k \omega^{\prime 2}}{(k+1) s^{2}+k \omega^{\prime} s+(k+1) \omega^{\prime 2}}
\end{gathered}
$$

The bode plot of one of the PLL used in [12] is given here. The bandwidth of the PLL is $33 \mathrm{~Hz}$. It attenuates harmonics of $1 \mathrm{kHz}$ to $-30 \mathrm{~dB}$. However, the effect of PLL with other controllers and output filter needs to be investigated for crucial stability analysis (Figure 8).

\section{Damped passive filter topologies}

Different types of passive filters are used in converter-based renewable generation sources. The effectiveness of filter, particularly passive type, primarily depends on the grid strength and variation of grid impedance with time. LCL is the common type of filter used widely. The variation in grid impedance affects the performance 
of LCL filter. Hence, the design of LCL filter is a trade-off between robustness and damping of resonance. The effective impedance with simple PI controller is explained here with RL filter topology.

\subsection{R-L filter}

Applying KVL at the converter output gives

$$
V_{c}-V_{P O C}=I_{f g}\left(R_{f c}+S L_{f c}\right)
$$

Converter output is controlled by output current

$$
V_{c}=f\left(I_{f g}\right)
$$

With simple PI controller, the output becomes

$$
V_{c}=\left(k_{P}+\frac{k_{i}}{s}\right)\left(I_{f g}\right)
$$

Putting Eq. (20) into Eq. (18)

$$
\begin{gathered}
\left(k_{P}+\frac{k_{i}}{s}\right)\left(I_{f g}\right)-V_{P O C}=I_{f g}\left(R_{f c}+S L_{f c}\right) \\
\left(\left(k_{P}+\frac{k_{i}}{s}\right) I_{f g}\right)-I_{f g}\left(R_{f c}+S L_{f c}\right)=V_{P O C} \\
Z_{o}=\frac{\left(S^{2} L_{f c}+S\left(R_{f c}-k_{p}\right)-k_{i}\right)}{S} \\
Z_{o}=\frac{(S+\alpha)(S+\beta)}{S}(\text { considering } \alpha<\beta)
\end{gathered}
$$

Equation (25) shows that there are two zeros and one pole. First, the output impedance decreases at $-20 \mathrm{~dB}$ per decade up to first zero at $\alpha$. At $\alpha$, the impedance response becomes flat, and at $\beta$ the impedance starts increasing at $20 \mathrm{~dB}$ per decade (Figure 9). So, the response of integrated filter becomes similar to that of series resonance filter. The selection of $\alpha$ and $\beta$ depends on the parameter selection of $L_{f c}$, $R_{f c}, k_{P}$ and $k_{i}$. Bode plot for RL filter without and with PI current controller is given in Figures 10 and 11, respectively.

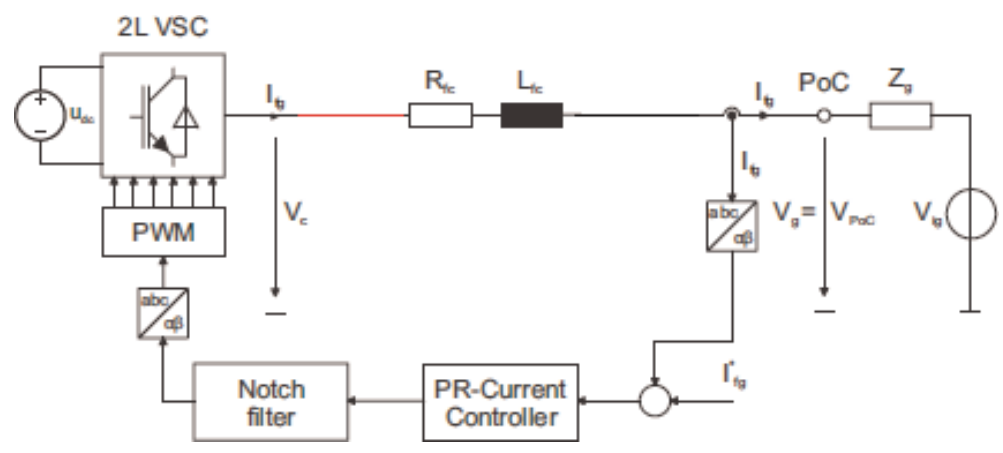

Figure 9.

Grid-connected inverter with filter. 


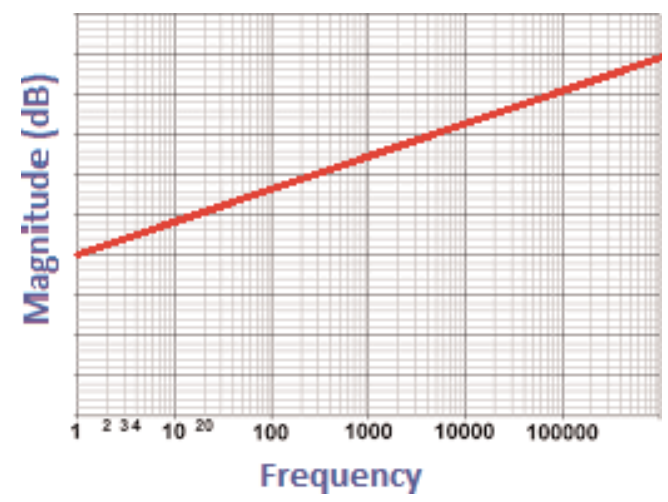

Figure 10.

Frequency response of impedance with RL filter without PI controller.

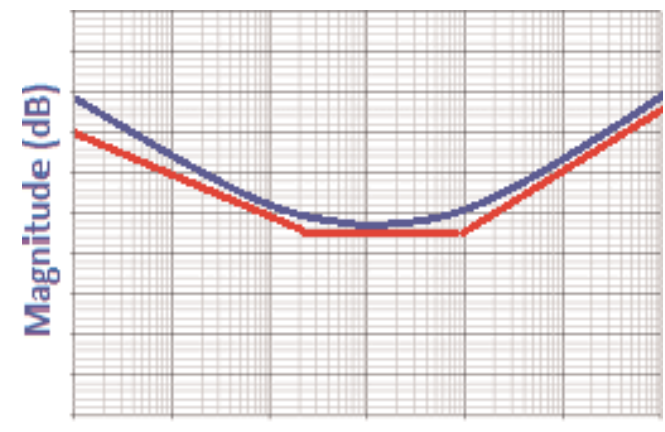

Frequency

Figure 11.

Frequency response of impedance with RL filter with PI controller.

\subsection{L-C filter}

LC filter is used in converters for industrial applications like variable frequency drive (VFD) and uninterrupted power supply (UPS). It is simple in construction and relatively less costly. The analysis of LC filter with PI controller is given here (Figure 12).

As per the standard practice, the value of $\mathrm{L}$ is selected such that its impedance at fundamental frequency should not drop by more than $3 \%$ of rated voltage. The capacitive reactance offers $1 / 5$ th of the fundamental inductive reactance at switching frequency of converter (around 3-4 kHz) to absorb harmonics effectively. Based on these criteria, LC filter is widely designed. The frequency response of LC filter with and without PI controller is given in Figures 13 and 14,

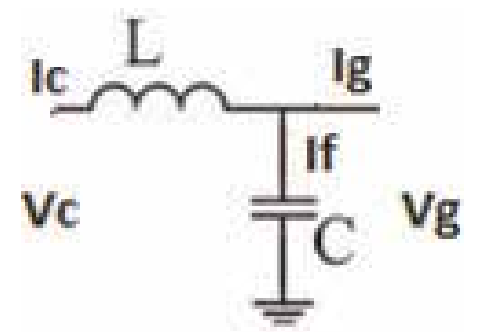




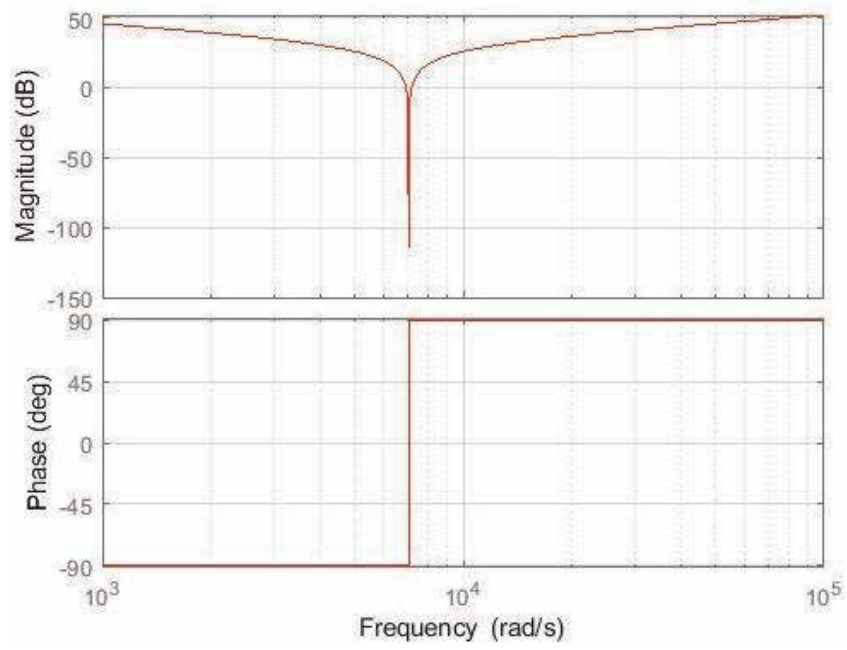

Figure 13.

Frequency response of LC filter without PI controller.

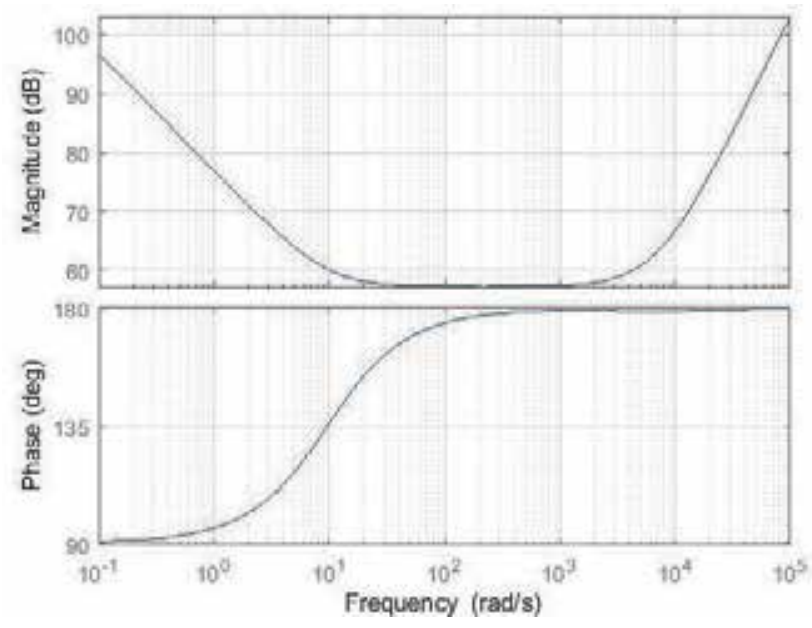

Figure 14.

Frequency response of $L C$ filter with PI controller.

respectively. It is clear from the difference in bode plot that PI controller changes the frequency response.

\subsection{L-C-L filter}

Initially LC filter was used for converter applications, but grid-connected inverter has unique requirements that LC filter may not provide. Properly designed LCL filter may overcome the drawbacks of LC filter (Figure 15).

Applying KVL,

$$
V_{c}=\left(\frac{\left(V_{g}+L_{g} s I_{g}\right)}{\frac{1}{s C_{f}}+R}+I_{g}\right) s L_{c}
$$


Harmonic Resonance Analysis for Wind Integrated Power System and Optimized Filter Design DOI: http://dx.doi.org/10.5772/intechopen.89167

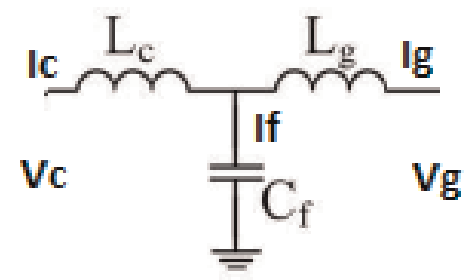

Figure 15.

$L-C-L$ filter.

The converter output voltage $V_{c}$ is a function of the grid current $I_{g}$. Then

$$
\begin{gathered}
V_{c}=f\left(I_{g}\right) \\
V_{c}=\left(k_{p}+\frac{k_{i}}{s}\right) I_{g}
\end{gathered}
$$

Putting this in Eq. (26) and simplifying further give

$$
Z_{0}=\frac{V_{g}}{\left(-I_{g}\right)}=\frac{a_{4} s^{4}+a_{3} s^{3}+a_{2} s^{2}+a_{1} s^{1}+a_{0}}{b_{3} s^{3}+b_{2} s^{2}+b_{1} s^{1}+b_{0}}
$$

where

$$
\begin{gathered}
a_{4}=L_{g} C_{f} k_{p} \\
a_{3}=L_{g} C_{f} k_{i}+R C_{f} L_{g} k_{p} \\
a_{2}=R L_{g} C_{f} k_{i}+L_{c} k_{p}-C_{f} \\
a_{1}=L_{c} k_{i} \\
a_{0}=0 \\
b_{3}=L_{c} C_{f} k_{p} \\
b_{2}=L_{c} C_{f} k_{i}
\end{gathered}
$$

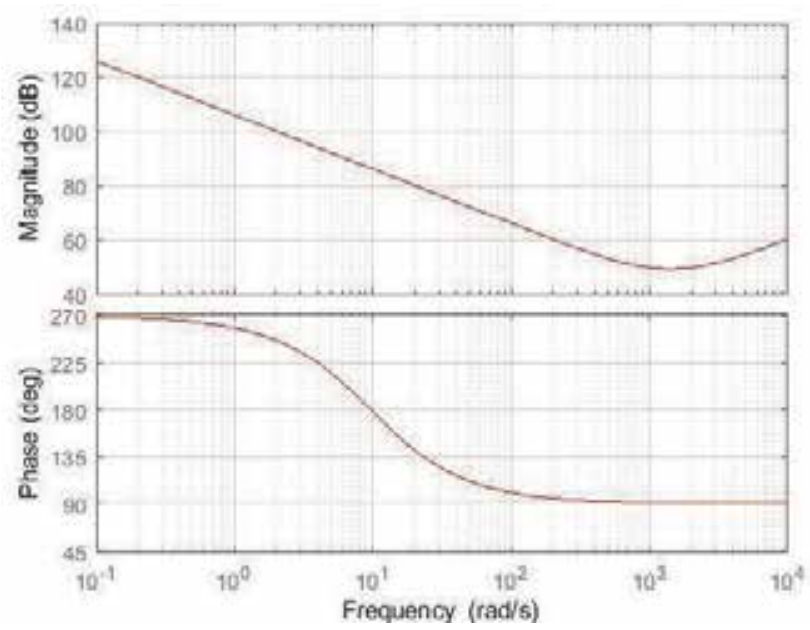

Figure 16.

Frequency response of LCL filter with PI controller. 


$$
\begin{aligned}
& b_{1}=0 \\
& b_{0}=0
\end{aligned}
$$

Figure 16 shows the frequency response of LCL filter together with PI controller. It is clear from Eq. (29) and from Figure 16 that the PI controller increases the order of filter, so the frequency response of passive filter gets changed by the controller action.

\section{Optimised filter design}

The design criterion for filter design should comply with the regulatory requirement. As per IEEE 519-1992 standard, the current harmonics for weak grid condition $\left(\mathrm{I}_{\mathrm{sc}} / \mathrm{I}_{\mathrm{L}}\right)$ should be less than $0.3 \%$. The ripple is caused by pulse width modulated signal. Output voltage varies from zero level to $\mathrm{DC}$ voltage level $\left(\mathrm{V}_{\mathrm{dc}}\right)$. The modulated wave causes ripple in the current, which can be reduced by proper selection of output filter parameters. Typical L-C-L filter is used in most of the inverter. The L1-C-L2 filter has three unknowns. The selection of these parameters depends on various factors. Grid condition is one of them. The strength of the grid decides the effectiveness of filters. Typical grid impedance varies from 5 to $8 \%$ with $\mathrm{X} / \mathrm{R}$ ratio in the range of 7-10 [13].

The switching frequency decides the ripple level and ripple frequency. Generally, inverter switching frequency remains in the range of $3-5 \mathrm{kHz}$. The ripple current is reduced either by increasing switching frequency or by using passive filter at the inverter/converter output. Higher switching frequency is selected to reduce the ripple current at the generation point, but it adversely affects the converter (IGBT) losses [14]. The second option is to use large inductor at the output of converter, but this not only incurs high cost but also increases the core losses. Typically $20 \%$ ripple current is expected in the output current. Keeping this in consideration, the inductor L1 is given by [7]

$$
L_{1}=\frac{1}{8} x \frac{V_{d c}}{\Delta I_{L} f_{s w}}=\frac{1}{8} x \frac{V_{d c}}{0.2 I_{\text {rated }} f_{s w}}
$$

The capacitor rating is selected such that the reactive power of capacitor is neither too high nor too low. Higher reactive power demands more power from converter, which causes more loss in reactor L1 and also more loss in converter switches. A lower value of capacitor will increase the inductor size. So, the capacitor is selected so that the reactive power should be in the range of $15-20 \%$ of the rated power.

$$
C=0.15 x \frac{P_{r}}{\omega V_{r}^{2}}
$$

\subsection{Passive damping of filter}

\subsubsection{Type I filter}

In LCL filter, the damping can be achieved by simply adding series resistance in series with capacitor $\mathrm{C}$. It is obvious that large value of damping resistance ( $\mathrm{Rd}$ ) gives large damping. But damping is effective around the resonance point only [15]. Above the resonance point, damping weakens. Also, the large value of resistance causes higher losses (Figure 17). 

DOI: http://dx.doi.org/10.5772/intechopen.89167

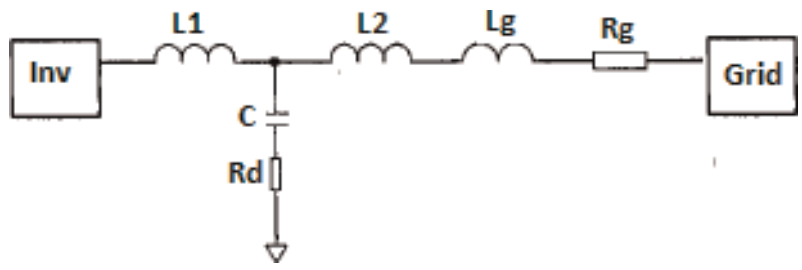

Figure 17.

Type I LCL filter.

Impedance of Type I LCL filter with damping resistance is given by

$$
Z_{o}=\frac{a_{4} s^{4}+a_{3} s^{3}+a_{2} s^{2}+a_{1} s^{1}+a_{0} s^{0}}{b_{2} s^{2}+b_{1} s^{1}+b_{0} s^{0}}
$$

where

$$
\begin{gathered}
a_{4}=L_{g} L_{1} C^{2} \\
a_{3}=L_{1} C^{2}\left(R_{d}+R_{g}\right) \\
a_{2}=L_{g} R_{d} C+L_{1} C \\
a_{1}=L_{g}+C R_{d} R_{g} \\
a_{0}=R_{g} \\
b_{2}=L_{g} C \\
b_{1}=\left(R_{g}+R_{d}\right) C \\
b_{0}=1
\end{gathered}
$$

Frequency response of LCL filter with damping resistor is given in Figure 18. The grid parameters are $\mathrm{Lg}=0.212 \mathrm{mH}$ and $\mathrm{Rg}=0.0095 \mathrm{Ohm}$, whereas the filter parameters are $\mathrm{L}_{1}=0.450 \mathrm{mH}, \mathrm{L}_{2}=0.300 \mathrm{mH}$ and $\mathrm{C}=270 \mathrm{uF}$. The damping resistance is varied from 0.05 to $0.8 \mathrm{Ohm}$. It is clear from the plot that the response of impedance is similar to inductive impedance. The notch is observed at the resonant frequency, which can be dampened by resistor in series with capacitor.

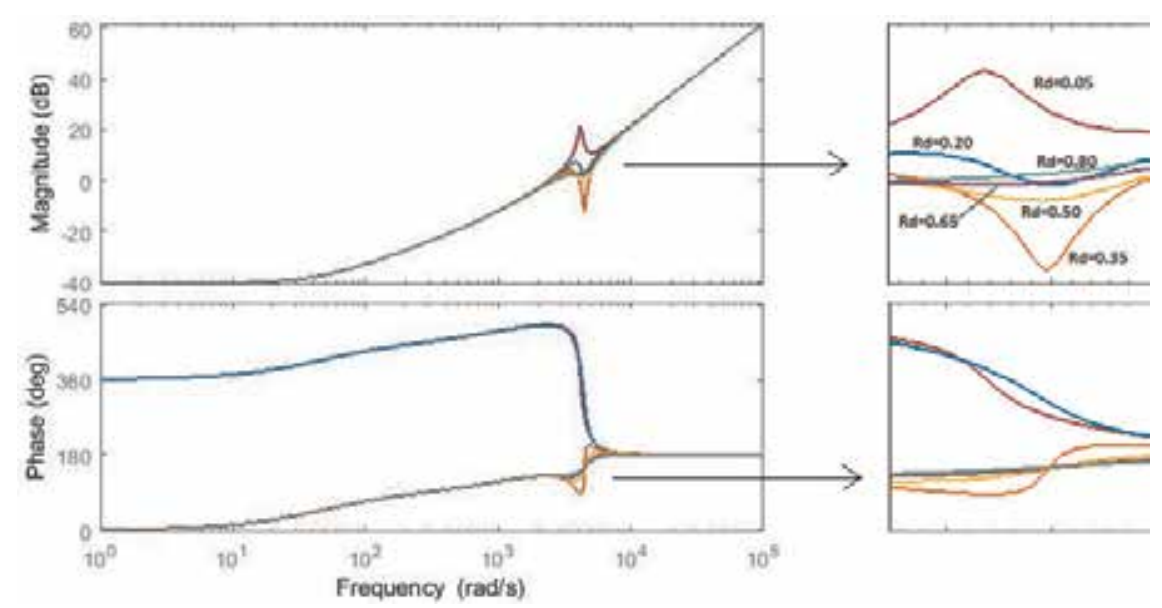

Figure 18.

Nyquist plot of Type I LCL filter. 
The gain margin is $-40 \mathrm{~dB}$ and phase margin is around $120^{\circ}$. So, as per the Nyquist criterion, the filter response is very stable.

\subsubsection{Type II filter}

In Type II LCL filter (Figure 19), the damping can be achieved by simply adding a parallel combination of resistor and inductor in series with capacitor $\mathrm{C}$. The effect of inductor is investigated using Nyquist plot. The value of $R_{f}=10 \mathrm{Ohm}$ and $\mathrm{L}_{\mathrm{f}}$ is varied from 50 to $500 \mu \mathrm{H}$. The effect of increase in $\mathrm{L}_{\mathrm{f}}$ observed using Nyquist plot is given in Figure 20.

Impedance of Type II LCL filter with damping inductor and resistance is given by

$$
Z_{o}=\frac{a_{4} s^{4}+a_{3} s^{3}+a_{2} s^{2}+a_{1} s^{1}+a_{0} s^{0}}{b_{3} s^{3}+b_{2} s^{2}+b_{1} s^{1}+b_{0} s^{0}}
$$

where

$$
\begin{gathered}
a_{4}=C L_{1} L_{f}\left(L_{g}+L_{2}\right) \\
a_{3}=L_{1} C L_{f} R_{f}+C L_{1} L_{f} R_{g}+C R_{f}\left(L_{2}+L_{g}\right)+C R_{f} L_{f}\left(L_{2}+L_{g}\right) \\
a_{2}=L_{1} L_{f}+C L_{1} R_{f} R_{g}+C L_{f} R_{f} R_{g}+L_{f}\left(L_{2}+L_{g}\right) \\
a_{1}=L_{1} R_{f}+L_{f} R_{g}+R_{f}\left(L_{2}+L_{g}\right) \\
a_{0}=R_{f} R_{g}
\end{gathered}
$$

Figure 19.

Type II LCL filter.

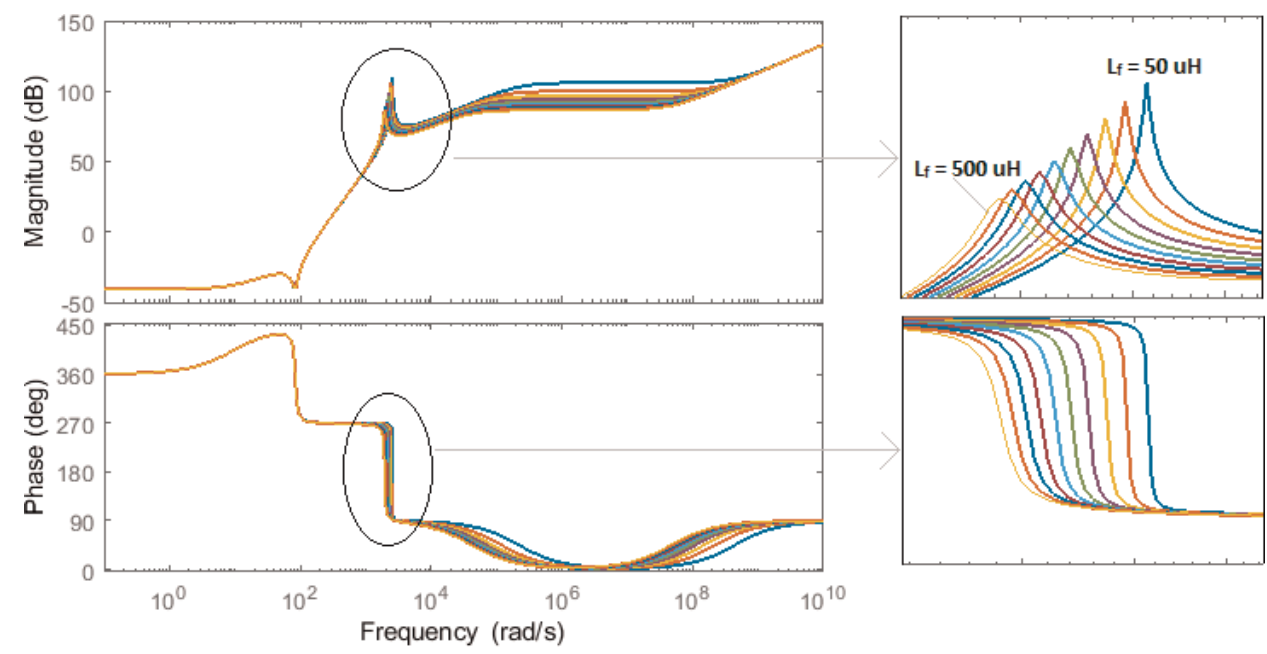

Figure 20.

Nyquist plot of Type II LCL filter. 


$$
\begin{gathered}
b_{3}=C L_{f}\left(L_{2}+L_{g}\right) \\
b_{2}=C L_{f} R_{f}+C L_{f} R_{g}+C R_{f}\left(L_{2}+L_{g}\right) \\
b_{1}=L_{f}+C R_{f} R_{g} \\
b_{0}=R_{f}
\end{gathered}
$$

From the bode graph, it is observed that the gain margin is $70-100 \mathrm{~dB}$ for various values of $\mathrm{L}_{\mathrm{f}}$. Similarly, the phase margin is $270^{\circ}$. As the value of $\mathrm{L}_{\mathrm{f}}$ is increased, there will be reduction in the gain margin.

\subsubsection{Type III filter}

In Type III LCL filter, the damping can be achieved by simply adding a parallel combination of resistor and inductor in series with capacitor $\mathrm{C}$. The effect of inductor is investigated using Nyquist plot (Figure 21).

Impedance of Type III LCL filter with damping inductor and resistance is given by

$$
Z_{o}=\frac{a_{4} s^{4}+a_{3} s^{3}+a_{2} s^{2}+a_{1} s^{1}+a_{0} s^{0}}{b_{3} s^{3}+b_{2} s^{2}+b_{1} s^{1}+b_{0} s^{0}}
$$

where

$$
\begin{gathered}
a_{4}=C C_{f} L_{1}\left(L_{g}+L_{2}\right)+C L_{1} L_{f} C_{f} \\
a_{3}=L_{1} C C_{f} R_{g}+C L_{1} C_{f} R_{f}+C_{f} L_{f}\left(L_{2}+L_{g}\right) \\
a_{2}=L_{1} C+C_{f}\left(L_{2}+L_{g}\right) R_{f}+C_{f} L_{f} R_{g} \\
a_{1}=L_{1} C_{f}+C_{f} R_{g} R_{f}+\left(L_{2}+L_{g}\right) \\
a_{0}=R_{g} \\
b_{3}=C C_{f}\left(L_{2}+L_{g}\right)+C L_{f} C_{f} \\
b_{2}=C C_{f} R_{g}+C C_{f} R_{f} \\
b_{1}=C_{f} \\
b_{0}=0
\end{gathered}
$$

Impedance shows two resonance points: first is parallel resonance and second is series resonance (Figure 22). At first resonance point, the output impedance increases, and at series resistance it is at the minimum value. The phase margin of filter is around $210^{\circ}$. This ensures the stability of filter. The resonant frequency will shift with change in grid resistance, so the damping effect is difficult to predict.

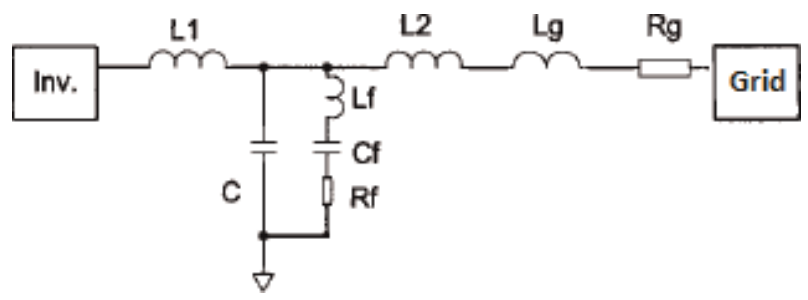

Figure 21.

Type III LCL filter. 


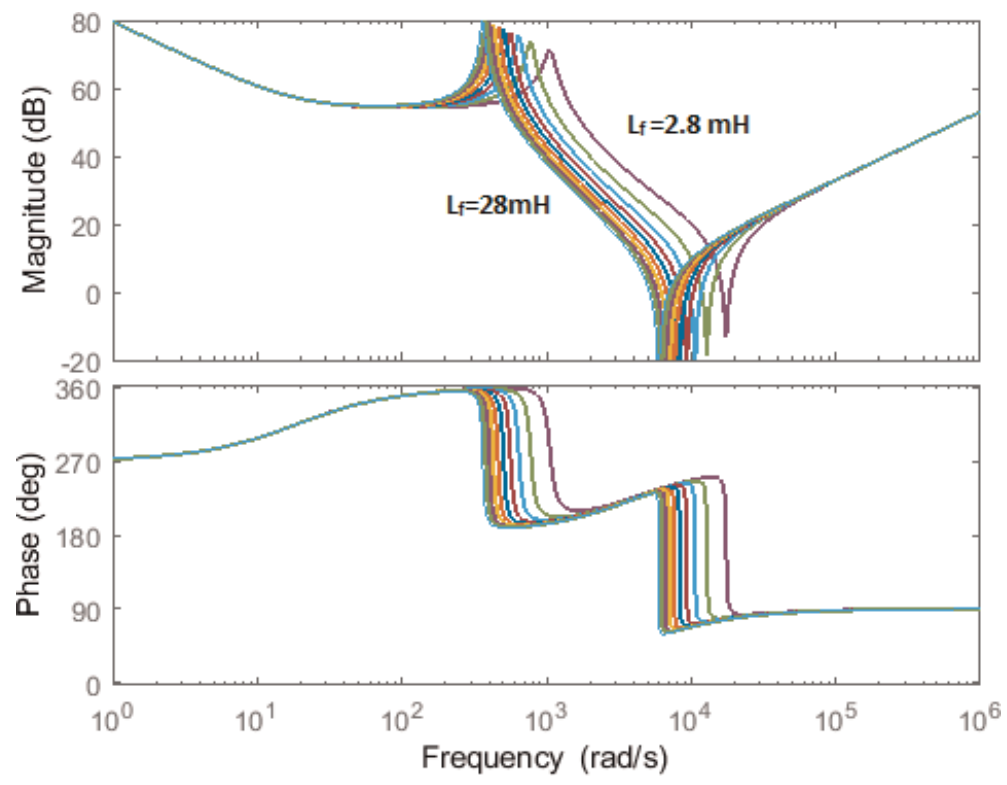

Figure 22.

Nyquist plot of Type III LCL filter.

\subsubsection{Type IV filter}

In Type IV LCL filter, the damping can be achieved by simply adding a parallel combination of resistor and capacitor Cf. The effect of resistor value is investigated using Nyquist plot (Figure 23).

Impedance of Type IV LCL filter with damping resistor $R_{f}$ in series with capacitor $\mathrm{C}_{\mathrm{f}}$ is given by

$$
Z_{o}=\frac{a_{4} s^{4}+a_{3} s^{3}+a_{2} s^{2}+a_{1} s^{1}+a_{0} s^{0}}{b_{3} s^{3}+b_{2} s^{2}+b_{1} s^{1}+b_{0} s^{0}}
$$

where

$$
\begin{gathered}
a_{4}=C C_{f} L_{1}\left(L_{g}+L_{2}\right) \\
a_{3}=C C_{f} L_{1} R_{f}+C C_{f} R_{g} L_{1} \\
a_{2}=L_{1} C_{f}+L_{1} C+C_{f} R_{f}\left(L_{2}+L_{g}\right)+C_{f}\left(L_{2}+L_{g}\right) \\
a_{1}=C_{f} R_{f} R_{g} \\
a_{0}=R_{g} \\
b_{3}=C C_{f}\left(L_{2}+L_{g}\right)
\end{gathered}
$$

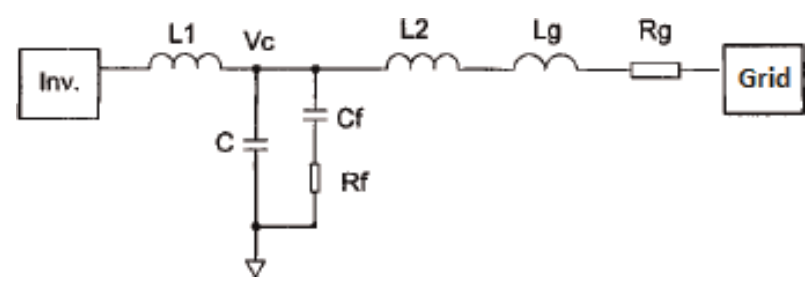

Figure 23.

Type IV LCL filter. 


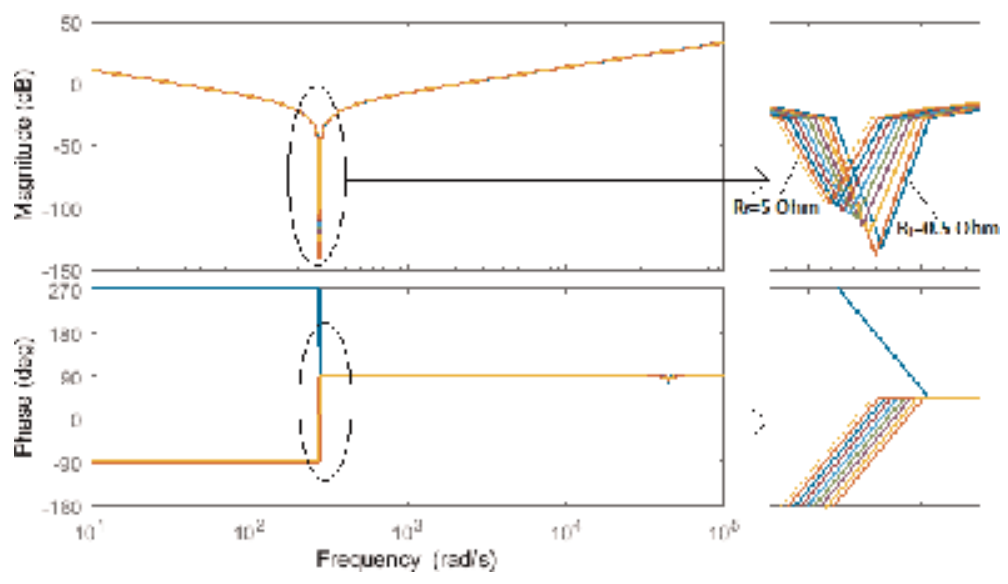

Figure 24.

Nyquist plot of Type IV LCL filter.

$$
\begin{gathered}
b_{2}=C C_{f} R_{g}+C C_{f} R_{f} \\
b_{1}=C_{f}+C \\
b_{0}=0
\end{gathered}
$$

The response of Type IV filter is similar to the tuned filter. The notch in frequency response of impedance is observed at the resonant frequency (Figure 24). This notch can be damped by putting higher value of resistor in series with the capacitor $\mathrm{C}_{\mathrm{f}}$. The phase of impedance sharply changes from -90 to $90^{\circ}$, which means the nature of impedance turns from capacitive to inductive. The ratio of $\mathrm{C}_{\mathrm{f}} / \mathrm{C}$ decides the damping effectiveness. The larger the $\mathrm{C}_{\mathrm{f}} / \mathrm{C}$ ratio, the larger will be the damping. Also, with increasing value of $\mathrm{C}_{\mathrm{f}} / \mathrm{C}$ ratio, the loss in resistor $\mathrm{R}_{\mathrm{f}}$ is also increases as more and more current tends to flow in it [16-18].

\subsubsection{Type V filter}

In Type V LCL filter, the damping can be achieved by simply adding a parallel combination of resistor and inductor with capacitor $\mathrm{C}$. The effect of inductor is investigated using Nyquist plot (Figure 25).

Impedance of Type V LCL filter is given by

$$
Z_{o}=\frac{a_{5} s^{5}+a_{4} s^{4}+a_{3} s^{3}+a_{2} s^{2}+a_{1} s^{1}+a_{0} s^{0}}{b_{4} s^{4}+b_{3} s^{3}+b_{2} s^{2}+b_{1} s^{1}+b_{0} s^{0}}
$$

where

$$
\begin{gathered}
a_{5}=C C_{f} L_{1} L_{f}\left(L_{g}+L_{2}\right) \\
a_{4}=C C_{f} L_{1} L_{f} R_{f}+C C_{f} L_{1} R_{f}\left(L_{g}+L_{2}\right)+C C_{f} L_{1} L_{f} R_{g} \\
a_{3}=C L_{f} L_{1}+C C_{f} L_{1} R_{f}+C_{f} L_{f} R_{f}\left(L_{g}+L_{2}\right) \\
a_{2}=L_{1} C R_{f}+L_{f}\left(L_{2}+L_{g}\right)+L_{f} C_{f} R_{f} R_{g} \\
a_{1}=L_{1}+R_{f}\left(L_{2}+L_{g}\right)+L_{f} R_{g} \\
a_{0}=R_{f} R_{g}
\end{gathered}
$$




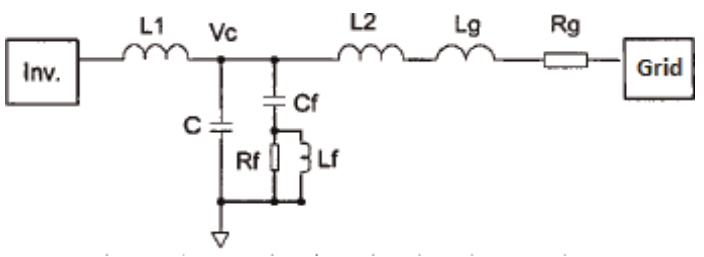

Figure 25.

Type V LCL filter.

$$
\begin{gathered}
b_{4}=L_{f} C C_{f}\left(L_{2}+L_{g}\right) \\
b_{3}=L_{f} C C_{f} R_{f}+L_{f} C C_{f} R_{g}+C_{f} R_{f}\left(L_{g}+L_{2}\right) \\
b_{2}=L_{f} C+C C_{f} R_{f} \\
b_{1}=R_{f} C \\
b_{0}=1
\end{gathered}
$$

Type V filter response shows two resonance frequency (Figure 26). The first resonance creates high voltage distortion, while the second resonance point gives rise to current distortion. If converter generates harmonics equal to this resonance, then there will be high voltage distortion and current distortion. However, with lower value of resistance, damping can be achieved. An additional inductor $\mathrm{L}_{\mathrm{f}}$ can reduce the resistive loss.

\subsection{Active damping of filter}

Grid-connected converters may not function stably under the harmonic resonance condition. Harmonic resonance occurs when the converter impedance and grid impedance becomes equal in magnitude and $180^{\circ}$ out of phase. The converter is generally connected to grid through filters, which is mostly LCL type. So, the parameters of LCL filters play an important role in keeping the successful functioning of converter. Also, the control structure of the converter shapes its output impedance. So, the control parameter should be selected such that it keeps the converter in the safe zone at all frequency. This is explained further here with analysis.

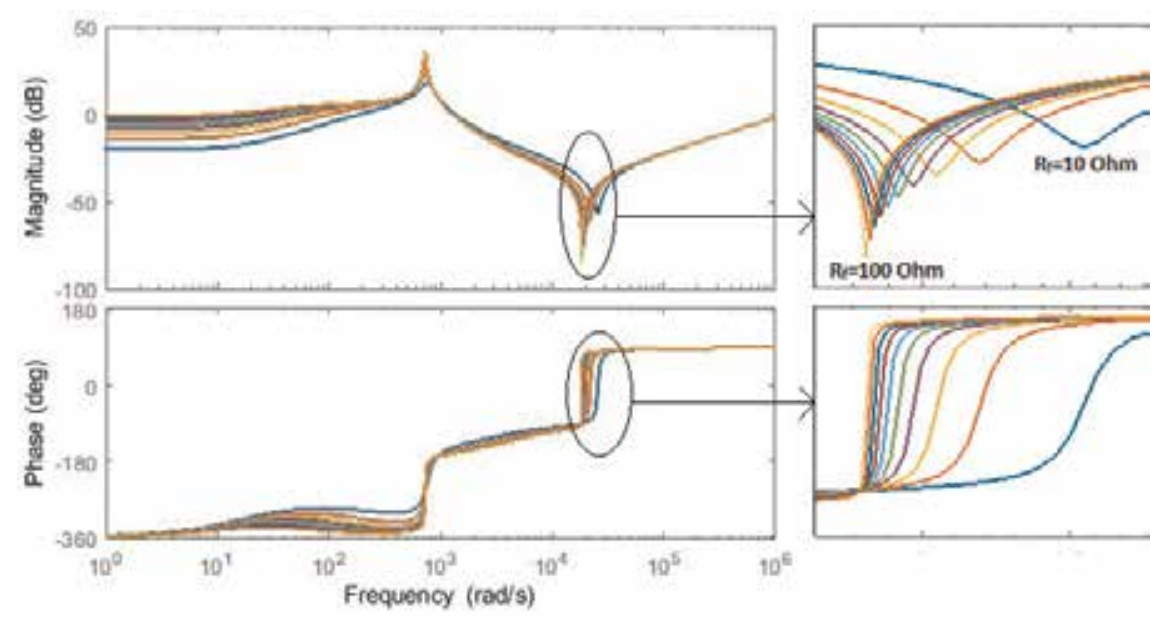

Figure 26.

Nyquist plot of Type V LCL filter. 
The inverter output characteristics depend on several factors like parameter of LCL filter, grid impedance, type of controller and control parameters. By selecting the appropriate parameter, the inverter can be operated with resistive output impedance, inductive output impedance and capacitive output impedance. Most of the inverters are operated with inductive (L-type) output impedance. This inverter is known as the L-type inverter. Here it is explained how an inverter impedance can be made capacitive (C-type inverter) with selection of virtual current control loop.

The output impedance of inverter is controlled by the virtual impedance. The single-line diagram of inverter with control loop is shown in Figures 27 and 28. The control is implemented with current loop and voltage loop, which gives good tracking behaviour and good output voltage. The feedback of current is taken from branch between the two capacitors. The first capacitor has a value of $\beta \mathrm{C}$, and the second capacitor has a value of $(1-\beta) C$. Thus, the overall value of capacitor is $C$. The output impedance of system after adding virtual branch $\mathrm{G}_{\mathrm{V}}$ is given by

$$
Z_{o}=\frac{\left(G_{I}+G_{V}\right) K_{P W M}+L_{s}+r}{C s\left(L_{s}+r\right)+C s(1-\beta) K_{P W M}\left(G_{I}+G_{V}\right)+G_{I} G_{U} K_{P W M}+1}
$$

The value of virtual impedance $G_{V}$ decides the overall impedance characteristics of output impedance. If $\mathrm{G}_{\mathrm{V}}$ is selected as per Eq. (38),

$$
\begin{gathered}
G_{V}+G_{I}=\frac{G_{I} G_{U} K_{P W M}+1}{C s \beta K_{P W M}} \\
Z_{0} \approx \frac{1}{C s}
\end{gathered}
$$

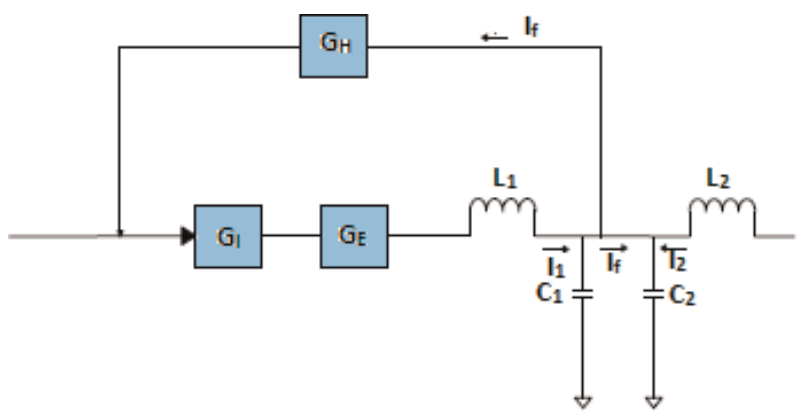

Figure 27.

Block diagram for active damping.

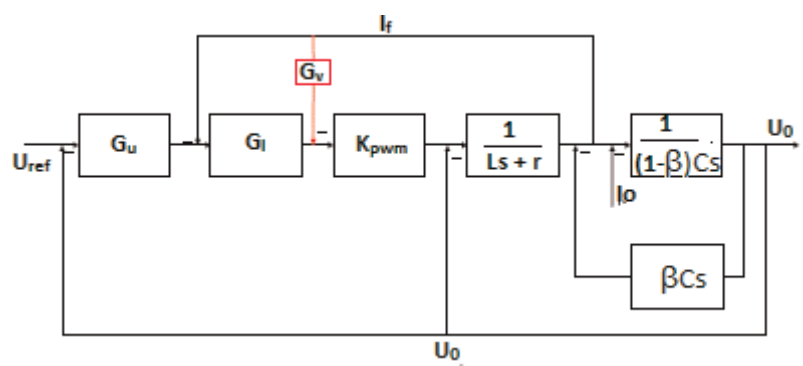

Figure 28.

Control diagram of active damping of filter. 
With the above value of $\mathrm{G}_{\mathrm{V}}$, the output impedance becomes capacitive. So, by selection of proper control loop and control parameter, the shaping of inverter output impedance can be effectively done. Also, the desired value of damping can be achieved. This method is known as active damping method. Like this, the inverter can be made either L-type or R-type.

\section{Conclusion}

The characteristics and response of impedance of grid-connected inverter depend on various factors like selection of output filter and its parameter, controller type and its parameters, structure of PLL, the delay in switching of converter, etc. In this chapter, using first-order PI controller and different controller types, it is explained in a simple way. The complexity of impedance increases with the order of the controller and its structure. Also, the reshaping of inverter impedance can be done by selection of suitable control structure. By adding virtual impedance, the output impedance can be made inductive, capacitive or reactive without adding any additional hardware. In this work different variants of passive filter are explained, and how their output impedance behaves at different frequency is also explained with the use of Nyquist plot. The active damping method is explained, whereby damping is achieved without using resistor in the filter, which is considered as a very energy-efficient method of achieving damping. The output impedance can be reshaped either by external hardware in the form of filter or by adding virtual control loop in the controller. The stability of converter depends on how converter output impedance interacts with the grid impedance. Numerous efforts have been made and can be found in literatures to achieve the stability only by reshaping the impedance. However, the stability region can be expanded by reshaping of impedance up to a certain extent only. Only reshaping of impedance does not guarantee the converter stability with changing grid condition. Also, the converter is more likely to fall in an unstable region with weak grid condition, but reshaping of impedance helps to prevent converter-based generation from going unstable. By analysing the output impedance in the frequency domain, the controller parameters can be adjusted to reshape the output impedance. By increasing the proportional gain of the PI controller, the magnitude of the output impedance can be increased and can enhance the ability of harmonic rejection. By increasing the integral gain of the PI controller, the phase of the output impedance can be increased to improve the stability of the system $[19,20]$ (Table 1 ).

\begin{tabular}{lc}
\hline Switching frequency & $4 \mathrm{kHz}$ \\
\hline Switching delay & $20 \mu \mathrm{S}$ \\
\hline $\mathrm{Lc}$ & $4 \mathrm{mH}$ \\
\hline $\mathrm{Lg}$ & $0.4 \mathrm{mH}$ \\
\hline $\mathrm{Cf}$ & $5 \mu \mathrm{F}$ \\
\hline $\mathrm{kp}$ & 1.2 \\
\hline $\mathrm{ki}$ & 11.6 \\
\hline
\end{tabular}

Table 1.

Converter parameter. 
Harmonic Resonance Analysis for Wind Integrated Power System and Optimized Filter Design DOI: http://dx.doi.org/10.5772/intechopen.89167

\section{Author details}

Jignesh Pravinbhai Patel and Satish Kantilal Joshi*

Department of Electrical Engineering, The Maharaja Sayajirao University of Baroda, Vadodara, Gujarat, India

*Address all correspondence to: skjoshi@ieee.org

\section{IntechOpen}

(C) 2019 The Author(s). Licensee IntechOpen. This chapter is distributed under the terms of the Creative Commons Attribution License (http://creativecommons.org/licenses/ by/3.0), which permits unrestricted use, distribution, and reproduction in any medium, provided the original work is properly cited. (c) BY 


\section{References}

[1] Blaabjerg F, Zhe C, Kjaer SB. Power electronics as efficient interface in dispersed power generation systems. IEEE Transactions on Power Electronics. 2004;19(5):1184-1194

[2] Ali MH, Wu B, Dougal RA. An overview of SMES applications in power and energy systems. IEEE Transactions on Sustainable Energy. 2010;1(1):38-47

[3] Flourentzou N, Agelidis VG, Demetriades GD. VSC-based HVDC power transmission systems: An overview. IEEE Transactions on Power Electronics. 2009;24(3):592-602

[4] Xia J, Fang X, Chow JH, Edris A, Uzunovic E, Parisi M, et al. A novel approach for modeling voltage-sourced converter-based FACTS controllers. IEEE Transactions on Power Delivery. 2008;23(4):2591-2598

[5] He J, Li YW. Generalized closed-loop control schemes with embedded virtual impedances for voltage source converters with LC or LCL filters. IEEE Transactions on Power Electronics. 2012;27(4):1850-1861

[6] Sun J. Impedance-based stability criterion for grid-connected inverters. IEEE Transactions on Power Electronics. 2011;26(11):3075-3078

[7] Blaabjerg F, Teodorescu R, Liserre M, Timbus AV. Overview of control and grid synchronization for distributed power generation systems. IEEE Transactions on Industrial Electronics. 2006;53(5):1398-1409

[8] Middlebrook RD. Input filter considerations in design and application of switching regulators. In: Proceedings of the IEEE-IAS Annual Meeting; 1976. pp. 366-382

[9] Freijedo FD, Yepes AG, Lopez O, Vidal A, Doval-Gandoy J. Three-phase
PLLs with fast postfault retracking and steady-state rejection of voltage unbalance and harmonics by means of lead compensation. IEEE Transactions on Power Electronics. 2011;26(1):85-97

[10] Wang F, Duarte JL, Hendrix MAM, Ribeiro PF. Modeling and analysis of grid harmonic distortion impact of aggregated DG inverters. IEEE Transactions on Power Electronics. 2011;26(3):786-797

[11] Beres RN, Wang X, Blaabjerg F, Liserre M, Bak CL. Optimal design of high-order passive-damped filters for grid-connected applications. IEEE Transactions on Power Electronics. 2016. DOI: 10.1109/TPEL.2015.2441299

[12] Sun J. Small-signal methods for AC distributed power systems-A review. IEEE Transactions on Power Electronics. 2009;24(11):2545-2554

[13] Li X-Q, Wu X-J, Geng Y-W, Zhang Q. Stability analysis of gridconnected inverters with an LCL filter considering grid impedance. Journal of Power Electronics. 2013;13(5)

[14] Jiao J, Nelms RM. Regulating output impedance using a PI controller to improve the stability of a single phase inverter under weak grid. In: 2016 IEEE 16th International Conference on Environment and Electrical Engineering (EEEIC); 2016

[15] Yang W, Wang M. Impedance modeling and output impedance coupling analysis of three-phase gridconnected inverters. In: 2018 IEEE International Power Electronics and Application Conference and Exposition (PEAC); 2018

[16] Zhong Q-C, Zeng Y. Control of inverters via a virtual capacitor to achieve capacitive output impedance. 
IEEE Transactions on Power

Electronics;29(10):5568-5578. DOI:

10.1109/tpel.2013.2294425

[17] He Y, Lai C-t, Chung S-h, Zhang X, $\mathrm{Wu} \mathrm{W}$. Use of series negative impedance to cancel the effect of equivalent grid impedance on the grid connected inverter in the DPGS. In: 2018 IEEE Applied Power Electronics Conference and Exposition (APEC); 2018

[18] Zhong Q-C, Zeng Y. Universal droop control of inverters with different types of output impedance. IEEE Open Access. 2016. DOI: 10.1109/

ACCESS.2016.2526616

[19] Messo T, Luhtala R, Aapro A, Roinila T. Accurate impedance model of grid connected inverter for small signal stability assessment in high-impedance grid. In: 2018 International Power Electronics Conference (IPEC-Niigata 2018 -ECCE Asia); 2018

[20] Ling P, Peng Z, Chao G. An impedance reshaping control strategy to enhance adaptability to grid for gridconnected inverters. In: 2018 China International Conference on Electricity Distribution (CICED); 2018 

Section 2

\section{Hybrid Technologies: Wind, Solar and Storage Systems}





\title{
A Review of Hybrid Renewable Energy Systems Based on Wind and Solar Energy: Modeling, Design and Optimization
}

\author{
Salisu Muhammad Lawan and Wan Azlan Wan Zainal Abidin
}

\begin{abstract}
In this chapter, an attempt is made to thoroughly review previous research work conducted on wind energy systems that are hybridized with a PV system. The chapter explores the most technical issues on wind drive hybrid systems and proposes possible solutions that can arise as a result of process integration in off-grid and grid-connected modes. A general introduction to wind energy, including how wind energy can be harvested, as well as recent progress and development of wind energy are discussed. With the special attention given to the issues related to the wind and photovoltaic (Wind-PV) systems. Throughout the chapter emphasis was made on modeling, design, and optimization and sensitivity analysis issues, and control strategies used to minimize risk as well as energy wastage. The reported reviewed results in this chapter will be a valuable researchers and practicing engineers involved in the design and development of wind energy systems.
\end{abstract}

Keywords: renewable energy, wind, solar, hybrid energy, optimization, modeling, simulation, techno-economic

\section{Introduction}

According to recent scientific literacy works [1-3], about $78-80 \%$ of the world commercial energy comes from fossil fuels, such as, petroleum, coal and natural gas. Those high-carbon sources have negative effects in our environments, such as, effects on heath, land, air and rain. In view of that, the attention of most countries around the globe has been shifted to low-carbon energy. Renewable energy is naturally abundant resources, which can be harnessed without compromising future energy needs. Unlike fossil fuels, which depletes as time goes on. Renewable energy sources like wind, solar, biomass, wave and tidal are abundant sources that can produce clean energy. On recent time, series of renewable energy technology improvement has been witnessed, because the cost of generating electrical power is decreasing [4].

Although, renewable energy is considered as the new technology of generating electricity, the barrier associated with renewable is stochastic and unpredictable weather behavior. Its availability varies depending on the location. That is why, it is necessary to complement renewable with other sources like batteries. Because of 
this intermittent nature of renewable, single renewable energy source tends to be problematic in terms of energy yield and operational cost. Based on the aforementioned drawbacks, two or more renewable are being combined to form a hybrid renewable energy system (HRES). The main goal of doing this, or to improve electrical power production, to minimize cost, to reduce negative effects associated with burning fossil fuels and to improve the overall system efficiency.

In recent times, the integrated renewable energy system is gaining more attention, because a hybridized system can be efficiently applied to supply high efficiency and reliable electricity to the end-users, unlike a single-renewable source. A HERS can be applied in stand-alone or grid-connected modes. Stand-alone system must have a large storage to handle the load. While in a grid-connected mode, the storage can be small, and the deficient power can be acquired from the grid. It should be noted that, grid-connected mode must have a power electronic controllers for load sharing, voltage, harmonic, and frequency control. Thus HERS operating model is classified into Island mode where the generated electricity is consumed locally and grid connected mode where the renewable energy source is connected to the grid [5].

It is interesting, to note that, among the renewable energy sources, wind power is the fastest growing in terms of global annual and cumulative installed capacity. Wind energy is almost everywhere around the world. But the wind speed strength varies depending on the particular area. Wind energy can be operates during the day and night times, unlike other renewable.

Solar is the cleanest and most abundant renewable energy source available on earth (SEIA 2013). Solar energy can be defined as radiant light and heat from the sun and is harnessed by human using technologies.

The amount of energy harnessed from the sun is depending on radiation and scientists define radiation in two different ways which are energy in wave form (electromagnetic wave) or energy in particle form (photons). The electromagnetic radiation emitted from the sun has the wave length interval from $0.1 \mathrm{~nm}$ to $104 \mathrm{~m}$. However, $95 \%$ of solar energy reach earth with the intervals of $0.3-2.4 \mu \mathrm{m}$ only $[5,6]$. The photons are traveling through space at the speed of $3.0 \times 108 \mathrm{~m} / \mathrm{s}$ and each photon carry different amount of energy measured in electron volts. Photovoltaic (PV) is derived from two words: photo which means light and voltaic or volt means the unit of electric potential. PV or solar cells, also called the semiconductor that converts sunlight to direct current (DC) electricity. PV cell is typically a thin wafer which consists of an ultra-thin layer of phosphorus-doped (N-type) silicon on top of a thicker layer of boron-doped (P-type silicon). The p-n junction is created through the doping and the electrical field is created near the top surface of the cell. When a sunlight which carries photons strike the PV cell, the current is produced because the photons prompt the electrons flow from $\mathrm{n}$ - to $\mathrm{p}$-junction. A typical silicon PV cell will produce about $0.5 \sim 0.6 \mathrm{~V}$ under open-circuit condition regardless of size. The current produces is proportional to the intensity of sunlight striking the surface as well as the efficiency and size of the cell. The photovoltaic cell normally connected in series or parallel circuit to produce the desired amount of current. PV modules consist of PV circuits sealed in an environmentally protective laminate. PV module is the fundamental building blocks for a PV system. PV panels involve one or more PV module assembled as pre-wired, field-installable unit. Series of PV panels are called a PV array which is ready-installed unit for power generation. The performance of $\mathrm{PV}$ modules and array are basically rated according to their maximum power output (w) tested under Standard Test Conditions (STC). Standard Test Conditions are defined as a cell (module) operating temperature of $25^{\circ} \mathrm{C}\left(77^{\circ} \mathrm{F}\right)$ and incident solar irradiance level of $1000 \mathrm{~W} / \mathrm{m}^{2}$.

Because of the aforementioned reasons, this chapter focuses on design, modeling, optimization, control and sensitivity analysis carried out on a hybrid system based on wind energy with a PV system. 


\section{Pertinent terms}

The terms are technical in nature and there brief explanations will go a long way to concept discussion the hybrid renewable energy power system characteristics, thus, optimization, reliability, grid, micro grid, macro grid, diverse generation, hybrid energy system, and hybrid renewable energy system.

\subsection{Optimization}

The term optimization is defined as a process, act or methodology of making system design or decision functional or effective as possible according to Hong and Lian [6]. Two practical fundamental methods of optimization exist, thus, the metaheuristics and the simulation-based, will be further discussed in the section of optimization. In another perspective, it is reported that it is finding of an alternative with the highest achievable performance and most cost effective under some constraints through maximizing desired factors and minimizing the undesired ones. However, maximization means an effort to attain desired highest system performance, reliability outcomes regardless of cost and this perception is equally testified by Hong and Lian [6]. However, any practical optimization could be restricted by lack of full data or information, whereas, if some data are available while others are not then linear programming can be employed. Conversely, the optimal sizing of renewable power system components to increase their energy, capacity or performance, thus, providing power, reliability impact is considered optimization according to Kaabeche et al. [7]. Consequently, Power system hybridization is an infrastructural design exploration using optimization tools to configure hybrid renewable energy components to enhance the power reliability enabling zero or minimal loss of power supply probability (LPSP). Probability is the likely hood of getting optimal power supply reliability, and that, notwithstanding all depends on the power supply infrastructure redundancy status. Redundancy of power components can either be fully active or partially actively working with the system structure to allow smooth electric power supply distribution without interruption. Passive means the components are on standby and are only engaged at the point when component failure occurs. Subsequently, system reliability with active redundancy has smoother power supply that does not allow loss of power supply or allows only minimal loss than the passive redundancy reliability component.

\subsection{Reliability}

Dependable, consistent, trustworthy, and steadfast are always watch words for reliability term, and relating to energy delivery to consumer electric loads it means consistent qualitative power supply. The Power supply system is actually designed with redundancy and diagnostics in order to achieve power supply optimal reliability. Redundancy takes several concepts of the simple arithmetic, such as, $\mathrm{N}+1, \mathrm{~N}+\mathrm{N}$, or $2 \mathrm{~N}$ for maximum reliability such as system automation as expressed [8]. The ' $2 \mathrm{~N}$ ' redundancy element stands out to provide a better redundancy than the others; it means the two coefficient multiplied by existing components as ' $N$ ' size. Power supply consistency depends on availability of alternative energy sources and backups such as energy storage subunits as applicable to hybrid renewable energy power supply.

The term hybrid in its self means a mixture of two different components with about similar results for a specific purpose, for example, a hybrid solar-micro hydro renewable energy source (HRES) to supply a rural community with reliable electricity. Conversely, the solar resource is understood to be stochastic by nature and it is the characteristics unlike micro hydro, so they function better when hybridized or 
designed to work complementing each other. The hybrid resources, improve upon by attaching hybrid energy storages so that the energy converters are supported by energy storage in an effort to attain zero loss of power supply probability (LPSP) [9]. In addition, the preceding hybrid capacity is extended by integrating electronic controller automation in the style of PLC/SCADA. Any consistency in power supply obviously means there should be insignificant loss of power supply probability (LPSP) at any given time in the operation of the system. The probability that a system delivers consistent qualitative output power over long time period is considered to be reliable as interpreted by this author [6]. Hence, a reliable system is characterized by dependable power supply expressed mathematically in terms of mean time before failure (MTBF), and arithmetically expressed as reliability.

$$
R=e^{(-t M T B F)}
$$

However, the mean time before failure is the predicted elapse time between operating system inherent failures using a Monte Carlo tool of simulation for reliability optimization and Perturb and Observe for maximum power point tracking for (MPPT) optimization.

Dependence on the provision of adequate components spare in case of urgency by employing redundancy is a healthy technical strategy attracting extra intervention costs. Plainly, idleness is what redundancy means and though there are two types, the passive and active types synchronized with the system, whereas, the passive types are most times in the standby mode waiting to be called upon for activity as explained by. Thus, the design of hybrid renewable energy resources structure, hybrid energy conditioner, hybrid energy storages and hybrid control platform for automatic energy management.

\subsection{Macro grid}

The physical framework of which electric power is produced using diverse generators to supply the load using electric conductors and cables overhead or underground transmission and distribution networks, usually use assorted transmission and distribution equipment. The Figure 1 represents electric power of three phase grid emanating from the generating station through step up transformer connected to three phase transmission lines and connected to step down transformer that distributes electric power to various consumer loads as explained by Diaf et al. [10]. The grid in Figure 1 is a three phase alternating current network for alternating current $(\mathrm{AC})$ loads, and conversely, a direct current (DC) model are also use for diverse loads as observed by Rao [11] Figure 1. Illustrates multiple grids made available and categorized as micro, and large networks connected to diverse conventional and renewable energy generators.

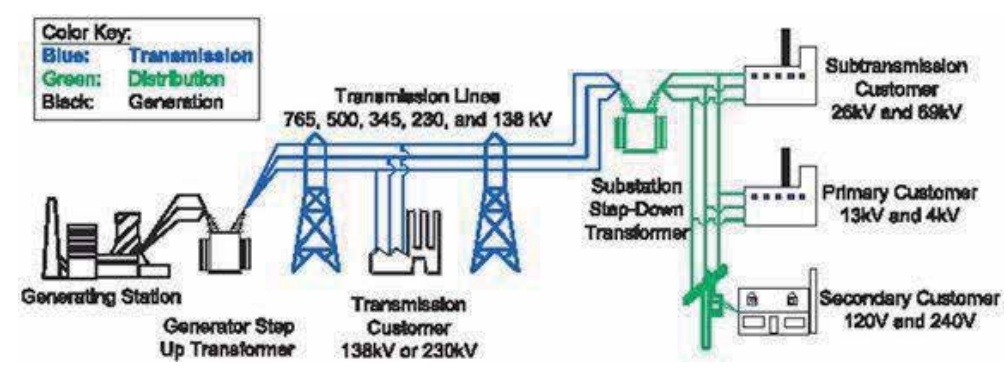

Figure 1.

Electric power multiple grids (transmission and distribution). 
A Review of Hybrid Renewable Energy Systems Based on Wind and Solar Energy: Modeling... DOI: http://dx.doi.org/10.5772/intechopen. 85838

\subsection{Micro grid}

Micro grid is said to be a network designed to integrate distributed energy resources, control operation, power quality issue, and energy management infrastructure for load demand and power supply stability. It is the smallest facility common among the grid with diverse generators using extra low voltage three phase-four wire supply down to single phase three wire or two wires supply configuration to provide electricity supply to consumers load. Furthermore, micro grid is considered to be a group of distributed resources (DER) and loads forming an electrical network. It has a grid of low-voltage distribution energy resource (DER), energy storage system (ESS) and/or micro sources such as photovoltaic, fuel cell, wind turbine, etc. Micro grid may have controllable energy sources such as biomass, hydro, fossil fuel or uncontrollable energy sources like solar and wind or may be flow-of-the-river that is dependent on daily, monthly and annual rain falls. Controlling solar and wind-micro grid is challenging it involves measuring the parameters such as solar irradiance or Insolation, PV array voltage, PV array current, ambient temperature, wind speed, and AC load pattern for a year evaluation. Conversely, the renewable energy storage loss, energy converter average yield, peak period parameters are used for evaluation of micro grid power performance as reported by Mohammadi et al. [12].

\subsection{Diverse generations (DGs)}

A single source of electric power delivery to the consumer, local load is a diverse generation strategy such as conventional fossil fuel generation like oil, coal, etc. or renewable energy method such as solar, wind, hydro, biomass, geothermal, etc. Diesel or gasoline generators that are usually and commonly use in the rural areas are all categorized as small diverse electric generators' power sources. The diverse generator located by left of the figure and representing either of conventional or renewable energy diverse source according to this author [13]. The integration of diverse energy sources for the operation, control, power management in real time power system make up a micro grid or network as positioned by Ashourian et al. [14]. Obviously, micro grid has distribution structure like the macro grid except that it is a smaller size, in a tiny network, and has a low power capacity. Diverse generation is made into a hybrid design mix whenever the hybrid energy storage is integrated into the micro grid structure to reliably evacuate power to the load.

\subsection{Hybrid energy system (HES)}

It will be good to start with hybrid energy system (HES). Hybrid energy system is the engineering design of hybridizing power supply components or pairing them, for example, arranging diverse energy resources to work in parallel (equivalent) is very common in power. So, hybridizing is defined as forming crossbreed of pairs of agent for working together to achieve a purpose. Thus, hybridizing is to manually or automatically synchronize two or more electric power generator resources or components to supply electric power to the grid, therefore forming hybrid energy system. Hybrid energy system is an infrastructural design that integrates diverse or multiple energy converters to energy storage, energy conditioners, energy management system. By and large hybrid renewable energy system (HRES) is an extension of HES that uses mix diverse resources as hybrid or all hybrid renewable energy resources to supply the electric power system.

The concept of the hybrid RE power system is the perception to implement reliability portfolio to avert LPSP that will affect the quality of power supply resulting in dynamic change and transient. Hence, reliability is the dependability of systems 
or components to be able to function appropriately under stated conditions for a specified period without failure. Furthermore, reliability is said to be a probability of success, expressed as reliability (R) equal to "1" minus (Pf) probability of failure i.e., $R=1-P_{f}$. Hence, reliability relates to safety factors and cost factor caused by system downtime, cost of equipment repairs, spare parts, personnel, and cost of warranty claims. High reliability level will of course result from good engineering, reliability concept such as employing the concept of electric power system design optimization. Stochastic parameter dynamics in power supply do affect system reliability as failure is unabated, unless the concept of hybridization is embraced and integrated in the power supply structure as stated [13]. Redundancy is provision of more than one alternative resource power supply or system component to perform certain task (important), duplication of active or passive subsystem, and complete energy storage backup integration in case of failure according to Ashourian et al. [14].

On the other hand, reliability covers several unique modus operandi which provides high quality output, affording utmost availability through redundancy, and advanced problem-solving capabilities of hybrid RE power system as stated by Mat et al. [15]. Thus, HES assume several design types such as multiple fossil fuel energy sources, diesel generator-SPV renewable energy sources or other hybrid renewable energy resources mixture. And the hybrid system reliability can be improved through the integration and optimization of essential components such as energy resources, energy storage and energy management. Hybrid energy renewable systems are economical, less or no fossil fuel consumption for all RER, and have no or less greenhouse gas emission. Solar, hydro and other renewable energy sources are environmentally safe and have adequate power generation potentials. Therefore the integration of these sources with energy storage as hybrid system has economic returns as supported by Mat et al. [15].

\subsection{Hybrid renewable energy power system (HREPS)}

Hybrid renewable energy power system (HREPS) is a cross breed or mixture of matching (parallel) power system infrastructure designed to offer power supply reliability. Hybrid renewable energy power system (HREPS) has enormous designs or models that consists of five common subunits, namely, (i) renewable energy resource (RER) or energy harvester, (ii) electrical system (energy conditioners), and (iii) energy storages system (ESS), however, (iv) a common Bus and (v) electronic logic controller (ECS) is included for system management. Hence, HREPS has several designs of hybridizing by optimal selection of appropriate components that consists of energy harvester, electrical energy conditioner, ESS, common bus and electronic logic controller, however, all hybrid design emphases on hybridizing RER than any of the five components. Thus, adapting redundancy norm on all subunits in order to avoid loss of power supply probability (LPSP) is necessary in order to realize optimal design. The nomenclature hybrid renewable energy power supply (HREPS) design requires the following project proposal subunits to be, the hybrid renewable energy resource (HRER)-hybrid energy storage system (HESS)-hybrid energy conditioner (HEC)-hybrid energy management (HEMS) of four modules hybridized subunits. Each of the subunit is expected to complement its pair to compromise optimal design to be modulated and simulated using simulation-based optimization in order to achieve power supply reliability devoid of loss of power supply probability (LPSP).

\subsection{Hybrid feasibility factors (optimization and levelized costs of electricity)}

Hybrid renewable energy power system (HREPS) optimization is hereby defined by Giraud et al. [16] as finding the utmost feasible performance or the most cost 
effective approach under given constraints by maximizing desired factors and minimizing undesired ones. In the case of this design performance was considered priority so, energy reliability is more pleasant than its scarcity (energy shortages). For illustration, gasoline generators were the only electricity source in the remote and rural areas else the community had to live with lanterns. Consequently, maximization of the means to obtain the highest result for electricity provision is better regardless of the cost. Therefore, an act, process or methodology of making a design to function effectively for specific purpose is termed system optimization as from [17]. Two factors are the probable yardstick in determining the hybrid potential of any given site constraints, namely, the optimization and levelized costs of electricity (LOCE).

\subsection{Optimization}

Optimum or best methods to explore the hybrid renewable energy system for power supply reliability are enormous. The RE use has been historically, abundantly everywhere, omnipresent, free cost, and non-polluting characteristics leading to the increase of required storage capacity. A small hybrid system is understood to economical and may not meet the user load demand, whereas the large one can provide reasonable power, but it is expensive. Hence, optimal sizing of RE power system demand mathematical model of the system component characteristics using special techniques to extract maximum power from the models. Also, hybrid system has a complex control system due to the stochastic and multiple power harvesters, for example, the maximum power point (MPPT) technique employed in system SPV makes the system more complex [18]. This hybrid and MPPT approach is termed the optimization of the SPV stochastic power component to meet operational power supply demand.

In addition, optimization of hybrid renewable energy power systems has two techniques, the optimum tools or component based on site available energy resources and the sizing of the components, and use the appropriate control strategy that will [19] automate operation of the integrated hybrid system. Optimum HREPS design, configuration can be conducted using several optimization algorithms such as numerical, probabilistic and heuristic methods under some conditions as reported by these authors [11]. Whereas, feasibility factor is an index called localized cost of energy (LCOE) used to find cost of the average price of electricity produced by the HRES over its life. These variables include initial investment, development, capital, operation and maintenance, and fuel costs put together for costs analysis.

However, feasibility factors of using optimization are complex, nonlinear, and nonconvex because of the unique mixed constraints. Optimization approach are said to be fundamentally two, namely, the Simulation-based that is tedious, time consuming, prone to human errors and the metaheuristic method using multiple objectives involving cost, performance, supply-demand management, grid limitations, algorithms such as numerical, probabilistic and heuristic methodology as stressed by these authors [20,10]. Optimization provides economic, efficient, and reliable power supply alternative energy without LPSP. Several of hybrid renewable energy power system optimization concepts were listed in Table $\mathbf{1}$ in six groups. Their names are the graphical construction, probabilistic approach, deterministic approach, iterative approach, artificial intelligence, and software based (simulation-based) as stated by these authors [15]. However, a read-made HOMER software is a tool used to model hybrid configuration for optimization that emphasize on two factors, minimizing cost and maximizing performance constraints as asserted by Hong and Lian [6].

Next, search-based and Monte Carlo simulation (SMCS) is another optimization pattern use for HREPS and energy storage system (ESS) to check power supply 
reliability. The SMCS allow chronological behavior and reliability of HREPS to be evaluated through of series of simulated experiments for high power loads reported by Ekren and Ekren [21].

Each of the optimization techniques is considered unique because it has design elements that are most appropriate for its application in order to get optimal results. Artificial intelligence optimization consists of five subcategories, generic algorithm, particle swarm, fuzzy logic, artificial neural network, and hybrid model by Arabali et al. [22].

Perturb and Observe method is a conventional maximum power point tracking (MPPT) approach used in the energy conditioner subunit. It is said to be a global maximum point (GMP) because the combination of Perturb and Observe quickly searches for first local maximum point (LMP) and the particle swarm optimization (PSO) search for the global maximum point. Experimental report shows this method to be good for hybrid power system because it can track GMP with faster convergence time and better dynamic response than using just PSO alone according to Hakimi and Moghaddas-Tafreshi [23]. Hence, optimization has several approaches, some of them are hereby listed in Table 1, according to techniques and RE system elements under study.

A hybrid renewable energy system optimization and components sizing has found to be economically and reliably better in meeting all load conditions with minimum investment and operation cost. This was a disclosure of many research using genetic algorithm, particle swarm optimization, simulated annealing, ant colony algorithm and artificial immune system algorithm results as reported by these authors [23]. Figure 2 depicts a graphical representation of optimization showing that it possesses two edges, the energy production and the energy demand control, conversely, the objective function is optimal design reliability inclined toward its constraints. These constraints determine the energy inputs maximize performance and the other hands LOCE minimize costs by Boubekri [4].

Hybridizing diesel with renewable energy to demonstrate the potential of RE to replace diesel generator. HOMER software platform was used to study the load pattern and modeled for HOMER hybrid RE optimization. Hybrid solar-wind-DG

\begin{tabular}{llll}
\hline S/N & Optimization technique & Elements & Remarks \\
\hline 1 & Graphical construction & Battery and PV array & Use two parameters \\
\hline 2 & Probabilistic approach & $\begin{array}{l}\text { Performance of } \\
\text { hybrid system }\end{array}$ & $\begin{array}{l}\text { Based on statistical data collection } \\
\text { approach }\end{array}$ \\
\hline 3 & Deterministic approach & $\begin{array}{l}\text { Stand-alone PV with } \\
\text { battery bank }\end{array}$ & $\begin{array}{l}\text { Use an equation for determining } \\
\text { specific values with constant } \\
\text { parameters }\end{array}$ \\
\hline 4 & $\begin{array}{l}\text { Iterative approach: hill climbing, } \\
\text { dynamic programming, linear, and } \\
\text { multiple objective }\end{array}$ & $\begin{array}{l}\text { Hybrid-solar-wind } \\
\text { system }\end{array}$ & $\begin{array}{l}\text { Based on LPS to find possible } \\
\text { combination of solar-wind } \\
\text { combination }\end{array}$ \\
\hline 5 & $\begin{array}{l}\text { Artificial intelligence: generic } \\
\text { algorithm, particle swarm, fuzzy } \\
\text { logic, artificial neural network, and } \\
\text { hybrid model }\end{array}$ & $\begin{array}{l}\text { Hybrid solar-wind } \\
\text { system with battery }\end{array}$ & $\begin{array}{l}\text { Based on evolution technique } \\
\end{array}$ \\
$\begin{array}{l}\text { Software based: homer, and } \\
\text { developed GUI application software }\end{array}$ & All of the above & $\begin{array}{l}\text { Input file with all necessary } \\
\text { information is supplied. The } \\
\text { software takes care of other things }\end{array}$ \\
\hline 6
\end{tabular}

Table 1.

Possible optimization techniques. 


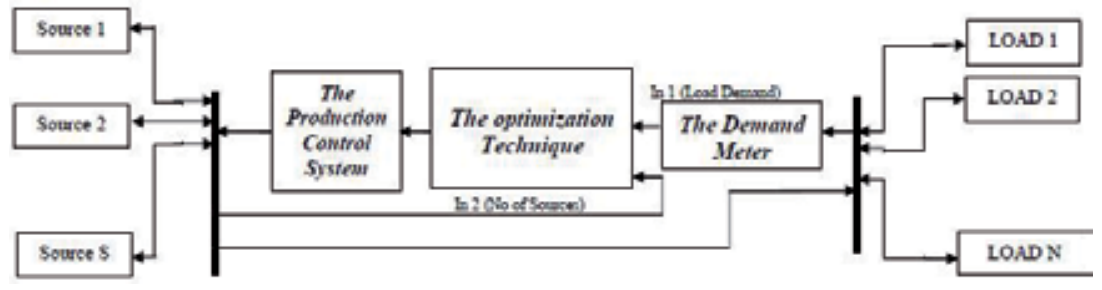

Figure 2.

Hybrid optimization, control and RE power production diagram [4].

were simulated to get four different technology models. The results show PV/hydro/ DG has the highest optimization value in comparison to diesel generator only, [6].

Renewable energy (RE) and hybrid energy system (HES) are expanding and the current design method is a simulation based optimization and meta-heuristic optimization methods. HES are medium scale application in remote areas and stand-alone, but they are needed for large scale integration to grid. HES are nonlinear, non-convex and composed of mixed variables that cannot be solved using traditional optimization methods. In the alternative, two approaches are used for optional HES design. Simulation based optimization and mete-heuristics optimization methods are limited in view of time consuming, rework, and error proneness analyzed by Arabali et al. [22]. From the onset, design of the hybrid power generation system (HPGS) begins with feasibility studies, analyze the potential and effectiveness using computer simulation as observed by Soysal and Soysal [41].

A systematic optimization methodology is to derive formulae hybrid RE system (HRES) Optimization by integration of demand response, day-ahead and real-time weather forecasting, and uploading model using a receding horizon optimization strategy is another approach. Practically demonstrated to a single family residential house HRES by Nfah et al. [24]. The demand-response and weather forecast methods are used to optimize the HRES in order to have minimize costs and maximize performance.

Furthermore, the state of the arts advanced generators; power electronic logic controller, grid requirements and control are optimized to improve wind power plant characteristics for efficient power delivery and integration according to Khan and Iqbal [25]. Consequently, the power electronic logic controllers, crossbreed SPV, hybrid ESS, and hybrid RER technology are therefore applicable to a solar photovoltaic power system for improved power reliability.

However, the approaches here consider optimization in terms of power supply reliability, but not only of the costs. Therefore, operating HREPS in the long run is economically preferable as costs are reduced no replacement reinvestment costs, fueling costs, maintenance costs, loss of power supply probability costs, and unquantifiable environmental degradation costs as economic parameters that indicate running diesel generator alone for power supply is bears exorbitant cost variables than operating hybrid REPS energy system.

\subsection{Levelized cost of energy (LCOE)}

Levelized energy cost (LEC) or (LCOE) is the unit-cost of electricity during the life period of power supply system in net present value (NPV) terms, often taken as alternative electricity average price to break even over generating system lifetime. However, LCOE is a general critical decision to proceed with a project development or not. There are two simulation models of the levelized cost of electricity (LCOE) available, namely, the EGC spread sheet and the system advisor model (SAM). 
LCOE is mathematically expressed as the life-cycle cost divided by lifetime energy produced interpreted as to break even. The renewable energy systems have higher initial capital costs outlay (ICC), it however amortizes in the long run with free natural fuel, less operation/maintenance costs and environmental friendliness free of GHG impact plus poisonous gases according to Refs. [25, 27]. Finally, localized cost of energy (LCOE) is an index use to cost average price of electricity produced by the HRES over its life initial investment, development, capital, operation and maintenance, and fuel costs as variables.

Hence, effective ways to cut energy cost are:

a. Cut down on development cost, capital cost, operation and maintenance cost

b.Energy production or increase life span of generation infrastructure. A model of wind generator life was improved to last between 20 and 25 years in Denmark, so this applies to other RE infrastructure as observed these authors [19]. However, informed decisions demand trade off in projects selection using pressing priority or objective functions to maximize.

Cut in operation costs includes fossil fuel, and then increase the price and global greenhouse gas emission concern has motivated hybrid renewable energy system standalone applications. Modeling, simulation and multi objective optimization decision tools supporting the leveraged cost of electricity (LCOE), life cycle cost (LCC), greenhouse gas (GHG) emission objective functions use to evaluate power supply reliability, optimization and market price sensitivity. However, it is difficult to justify LCOE and LCC of standalone RE components in rural electrification projects. Conversely, LEC, LCC and GHG fronts are simplified by pairing LCC-LEC and LEC-GHG for decision making according to Khan and Iqbal [25]. HOMER simulation reported that the LCOE of energy of optimized hybrid PV-Wind-diesel-battery is lower than hybrid energy system without renewable energy mix. Consequently, it was concluded that diesel generator supply alone is not feasible as fossil fuel price increases rapidly as reported by Ekren and Ekren [21].

Solar, wind and other renewable integration with energy storage as hybrid system has economic returns of LCOE of providing adequate power, environmental friendliness and reliability for all load conditions as supported by Nema et al. [26] Alternatively, three analyses model were put to test costs-benefits of solar PV, thus, short-run, medium-run and the long-run analyses. The short-run considers costseffectiveness on incremental increases, the medium-run focus on non-incremental change implications in solar capacity, whereas, the long-run dwell on carbon targets of the twenty-first century. Hence, economics depends on grid integration costs, low-carbon technologies and technological advances potential [27].

The common cost-effective criteria in photovoltaic phenomena rely on policy jurisdiction, frame work such as incentives like fit-in-tariff (FIT), tax credits, carbon reduction certificates variables motivates investors. Economically, return on investment (ROI) is always a prominent business yard stick and motivator for an investor. Conversely, feasibility study on solar PV indicated that it has long term high yield rate of return (ROR), see Table 2 give details as reported [28].

\subsection{Hybrid renewable energy power system (HREPS) bus}

Electricity bus is a good conductor in made into a bus-bar for transporting energy from power generator/converter to the grid. Hybrid renewable energy power system (HRES) has two levels of voltage bus the DC and AC produced by the mixture and crossbreed of energy resources. 
A Review of Hybrid Renewable Energy Systems Based on Wind and Solar Energy: Modeling... DOI: http://dx.doi.org/10.5772/intechopen. 85838

\begin{tabular}{lllll}
\hline S/No & PV type & $\begin{array}{l}\text { Payback period } \\
\text { range (years) }\end{array}$ & $\begin{array}{l}\text { Emission rate } \\
\left(\mathrm{gCO}_{2} \text {-eq/KWh }\right)\end{array}$ & Remarks \\
\hline 1 & Thin film & $0.75-3.5$ & $10.5-50$ & $\begin{array}{l}\text { Environmentally } \\
\text { friendly and suitable }\end{array}$ \\
\hline 2 & Mono-silicon & $1.7-2.7$ & $29-45$ & Ditto \\
\hline 3 & Advanced PV system technologies & & Lower than above rate & $\begin{array}{l}\text { Environmentally } \\
\text { friendly and suitable }\end{array}$ \\
\hline I & High concentration & $0.7-2.0$ & Higher than above rate & Ditto \\
\hline II & Hetero-junction & Ditto & - & Rate research is ongoing \\
\hline III & Dye-sensitized & - & &
\end{tabular}

Table 2.

Feasibility on PV types with payback period and environmental impacts.

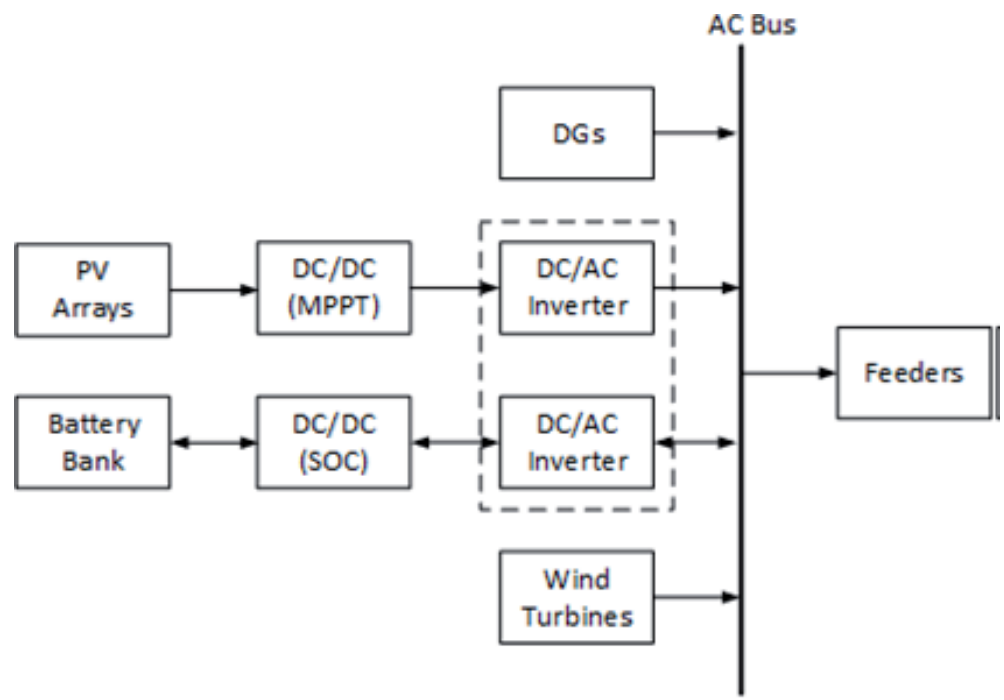

Figure 3.

Series hybrid RE power system with single AC bus for all AC load [15].

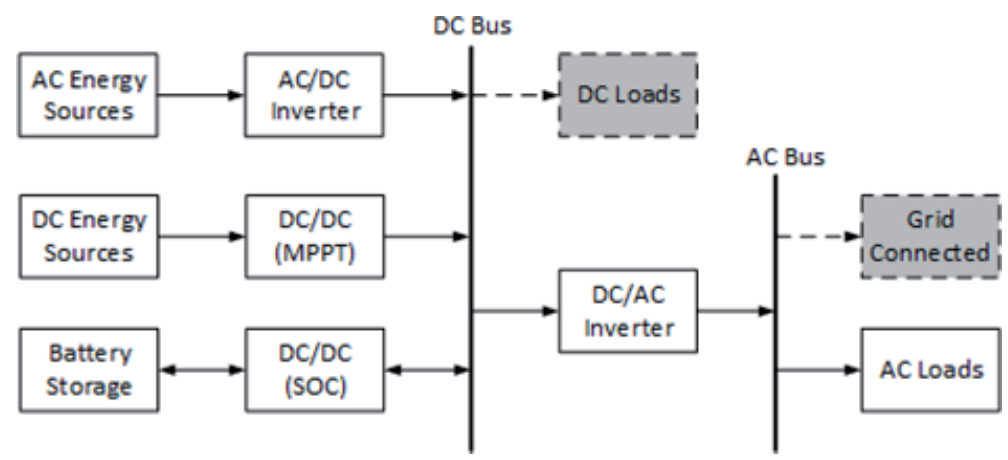

Figure 4.

Parallel (hybrid) RE power system with both AC and DC bus plus AC and DC loads [15].

The two levels AC and DC bus by extension are fundamentally two configurations, namely, the series and the parallel bus arrangements as shown in Figure 3 for the series connection, Figure 4 and Figure 5 for parallel bus as reported by Zhou 


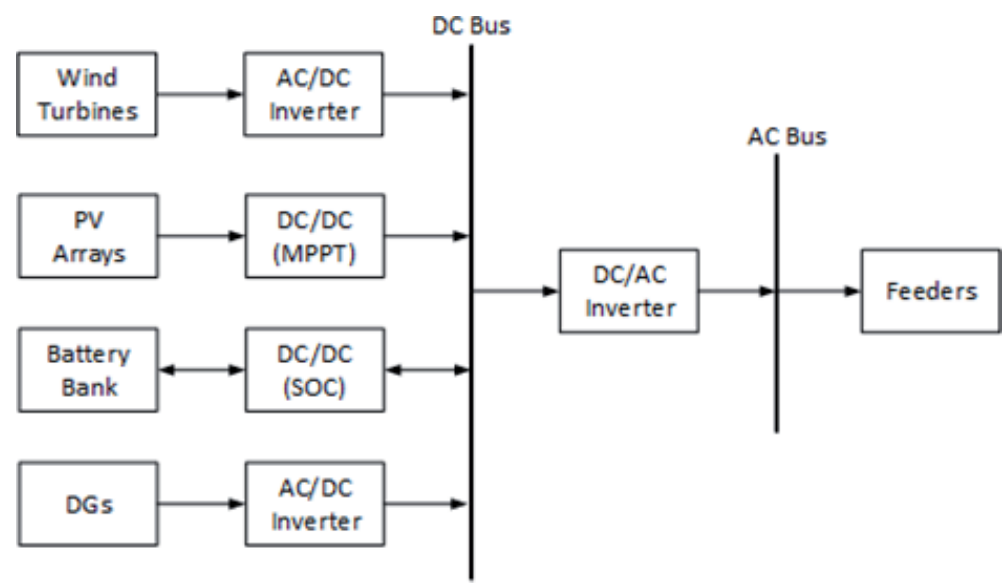

Figure 5.

Parallel hybrid RE system with both AC and DC bus for only all AC loads [15].

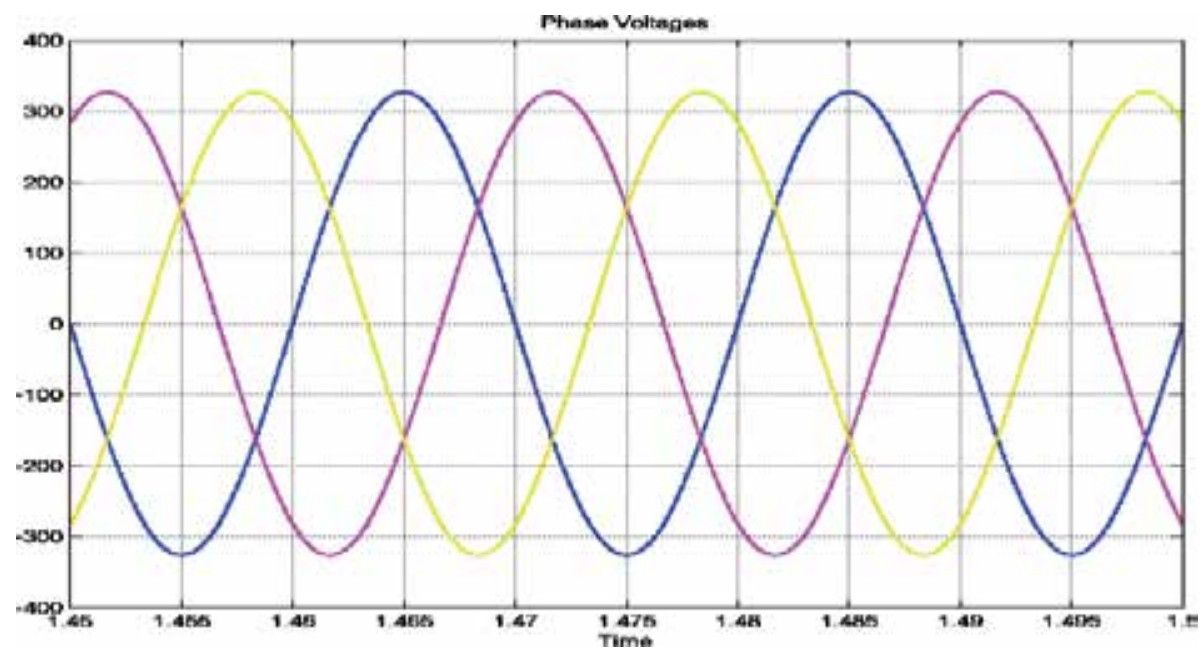

Figure 6.

Hybrid RE three phase output voltage waveforms [30].

and Sun [29]. These authors have illustrated the ideology of hybrid methodology can be synchronized to serve the electric load better, meaning mixing energy to improve the power of equivalent RE converter infrastructure to have reliability in power supply delivery.

The Figures 3-5 are in one line/block schematic representing three phase system for hybrid RE resources to produce output voltage waveform shown in Figure 6. However, hybrid RE can be also be designed to supply single phase system for smaller single phase load demand and its output voltage wave is single implied.

\subsection{Renewable energy resources (RER) optimal sizing}

Hybridizing is a common strategy for improving the sizing of renewable resource (RER) energy resources; it is also known as crossbreeding in the SPV. The optimum scale of renewable energy resource to harvest energy reliably depends on the optimal design of conversion model. Energy converters are RER and come with assorted characteristics, sizes, and brands guide in the design and the implementation of projects. The RER characteristics of solar photovoltaic and micro hydro are the main 
focal area to be considered for discussion and analysis in this study. Henceforward, RER as the name implies, are replenish able resource that naturally are regenerated in accordance with the climatic condition and topography of the locality of solar, hydro, and so on as established by Mohammadi et al. [12]. The common among the renewable energy resources are solar and hydro considering their technical and economic benefits as considered by Daut et al. [13]. Solar photovoltaic and micro hydro resources have several statistical variations in nature and are therefore are dependent on the peculiarity of specific geographical location's weather.

\section{Wind energy and photovoltaic systems}

From the deep literature survey conducted, a lot of studies are being done with divergent ideas and necessities on the possibility of integrating wind and PV system. The studies can be classified into, modeling, design, optimization, control and techno-economic strategies. On the other hand, some researchers proposed a stand-alone hybrid system, while others applied wind and PV system in grid connected mode.

\subsection{Modeling and design of PV-Wind system}

A lot of modeling and design of the PV and Wind have been developed using different approaches. The design can be categorized into two, it can be a grid or stand-alone. A grid PV-Wind system proposed by Harini et al. [31] used Wind generator, wind side converter, DC-DC converter, and grid interface inverter. The MPPT is used to optimize the DC voltage coming from the solar panels. The design was implemented in Matlab environment using Simulink. The schematic diagram of the overall system is shown in Figure 7.

PV-Wind hybrid system was used to generate electricity in Iraq; the planned system was simulated using MATLAB solver, where the input variables for the solver were the meteorological data for the selected areas and the sizes of PV and wind turbines. Outcomes revealed that it is achievable in Iraq to implement the solar and wind energy to come up with enough power for some communities in the desert or rural area. Additionally, it is feasible to use such a system as a black start source of power in the course of total shutdown time. Final results also showed that the desired place for this system is in Basrah for both solar and wind energy [32].

A Wind-PV-diesel hybrid power system is developed using HOMER software for a small town in Saudi Arabia which happens to be at the moment powered by a diesel power plant comprising of eight diesel generating sets of $1120 \mathrm{~kW}$ each, The

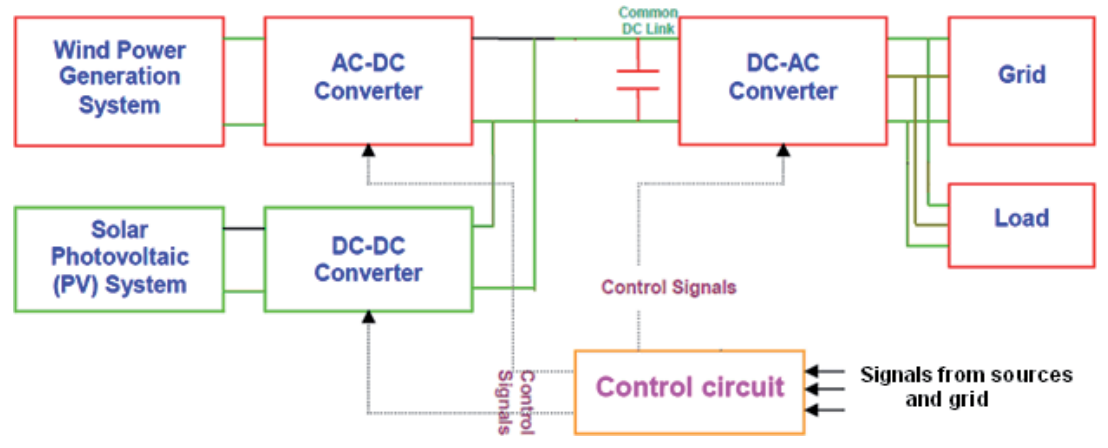

Figure 7.

Schematic diagram of a grid PV-Wind system. 
annual contributions of wind, solar PV and the diesel generating sets were 4713.7, 1653.5, and 11,542.6 MWh, respectively [33]. Performance of hybrid PV-Wind for hydrogen generation was studied in Sopian et al. [34]. The system consists of photovoltaic array, wind turbine, PEM electrolyser, battery bank, hydrogen storage tank, and an automatic control system for battery charging and discharging conditions. The system generated $130-140 \mathrm{ml} / \mathrm{min}$ of hydrogen, for an average global solar radiation and wind speed varying from 200 to $800 \mathrm{~W} / \mathrm{m}^{2}$ and 2.0 to $5.0 \mathrm{~m} / \mathrm{s}$ respectively. While authors [35] have design small-scale electric grid based on hybrid PV-Wind, the model is shown in Figure 8. Presented results shown that the coupling of subunits reduces annual grid power transfers by more than $10 \%$ and increases the renewable power contribution to the demand by almost 7\% [35]. To increase efficiency of PV-Wind hybrid system. Multi wind turbines and PV systems was successfully model in Mikati et al. [35]. The simulation outcomes revealed that the power end result of the wind turbines in multi-turbine wind-solar hybrid system improves by 18.69, 31.24 and 53.79\%, when used in Shenyang, Shanghai and Guangzhou, respectively, in comparison with the reference system [35]. In the work of [36] as shown in Figure 9, a special hybrid PV and Wind was used to power an UV (ultraviolet) water purification system. A 100-W solar-PV system that has a $500-\mathrm{W}$ wind turbine lead in pumping and filtering adequate water in order to meet the safe and clean water demands of 4000 people (16,000 1/day) at an approximated equipment expense of $\$ 4630$.

One work used [37] a new converter technology to implement a hybrid PV-Wind System using Matlab. The topology utilizes a combination of Cuk and SEPIC converters. This setting enables the two resources to provide the load independently or at the same time dependent on the availableness of the energy sources. Some design

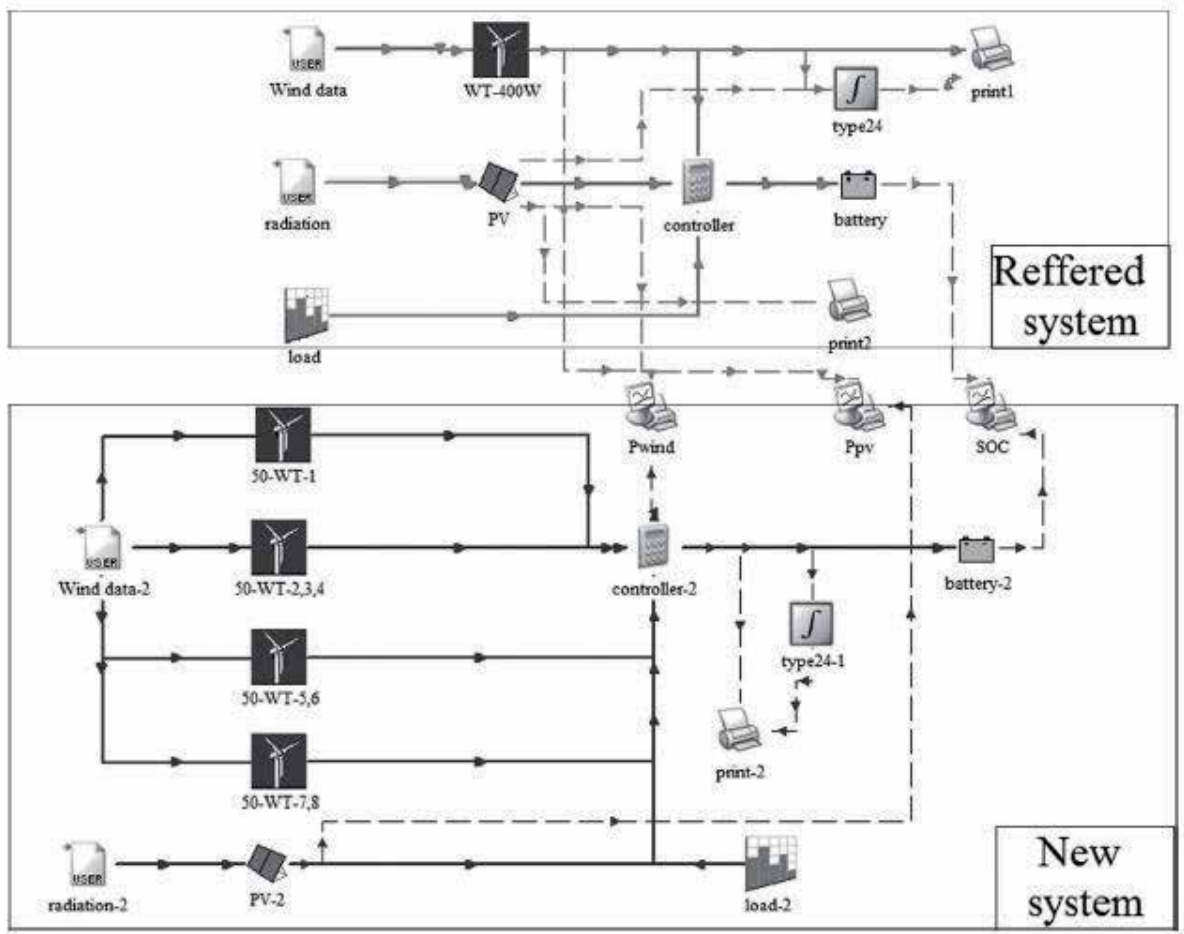

Figure 8.

Simulation models of the two hybrid systems. 


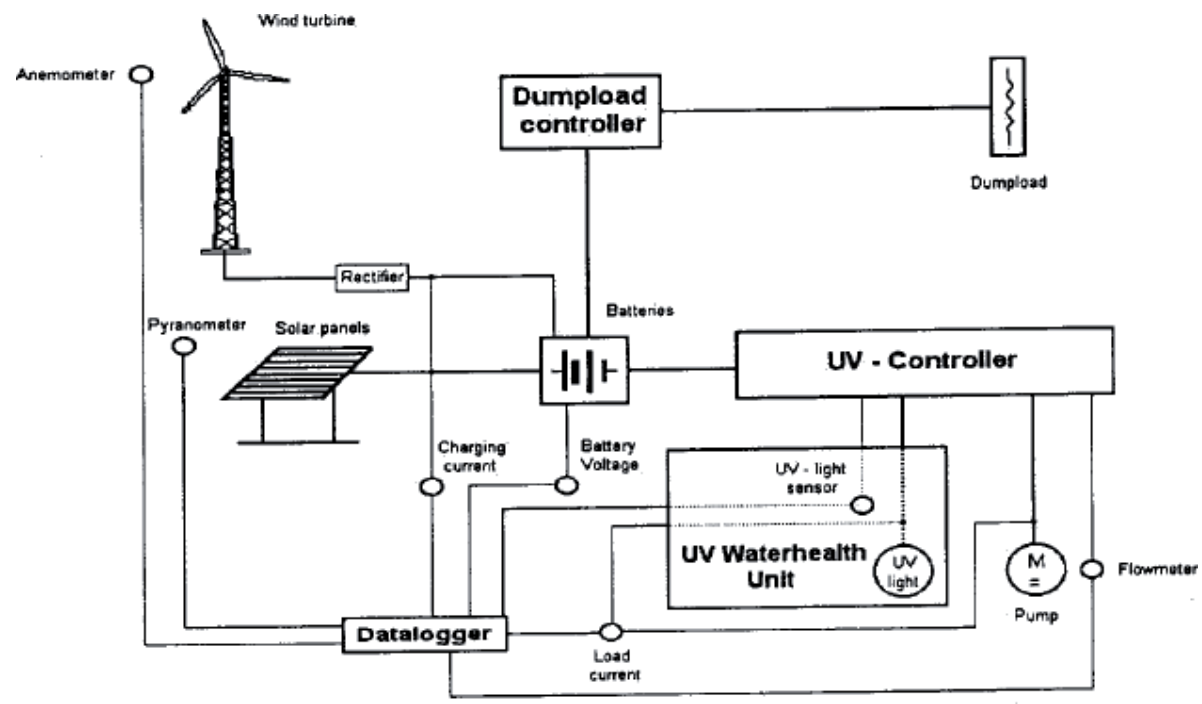

Figure 9.

Schematic of UV water filtration system.

includes control strategy for instance, the work of conducted by Moubayed et al. [38] reported a control of a hybrid solar-wind system with acid battery for storage. Steady-State Functionality of a Grid-Connected Rooftop Hybrid PV-Wind system was designed and tested by Kim et al. [39]. The design considers system consistency, power quality, loss of supply, and the effects of the randomness of the wind and the solar radiation on system. Limited studies are being done on micro generation based on PV-Wind, the best example case is a hybrid system with solar energy and wind energy for micro power production [40]. Residential hybrid PV-Wind was developed in [41]. While a PV-Wind with simple MPPT was implemented, the suggested system is desirable due to its convenience, convenience of control and affordable [42]. Few studies consider power generation to support national grid, example one study conducted in Jordan [43]. The need for additional energy tends to make us search for new energy sources. Authors in [44] designed a domestic solar-wind hybrid energy system as shown in Figure 10.

\subsection{Optimal sizing of stand-alone}

In more remote rural areas, PV and Wind system are widely used to supply electrical energy to consumers. Different methodologies have been applied in that regards. A methodology for optimal sizing of PV and Wind for stand-alone system is presented in [45]. The study aims at minimizing cost using genetic algorithm. The simulation outcomes validate that hybrid PV/WG systems feature reduced system cost when compared to the situations where either solely WG or exclusively PV sources are being used. The work of [46], considered optimization of PV/Wind based on number of solar panels ad wind turbines for minimal cost reduction. The findings of this study showed that optimum battery capacity, with optimum number of PV modules and wind turbines subject to lowest cost can be attained with high accuracy and reliability. One research conducted [12], used particle swarm optimization (PSO) algorithm for optimal sizing of PV and Wind system, though the study is limited to micro-grid system, however, energy storage was included. In Ref. [47], used discrete chaotic harmony search-based simulated annealing (SA) algorithm for optimum design of PV/wind hybrid system. The suggested method is employed to get the best possible design of a PV/ 


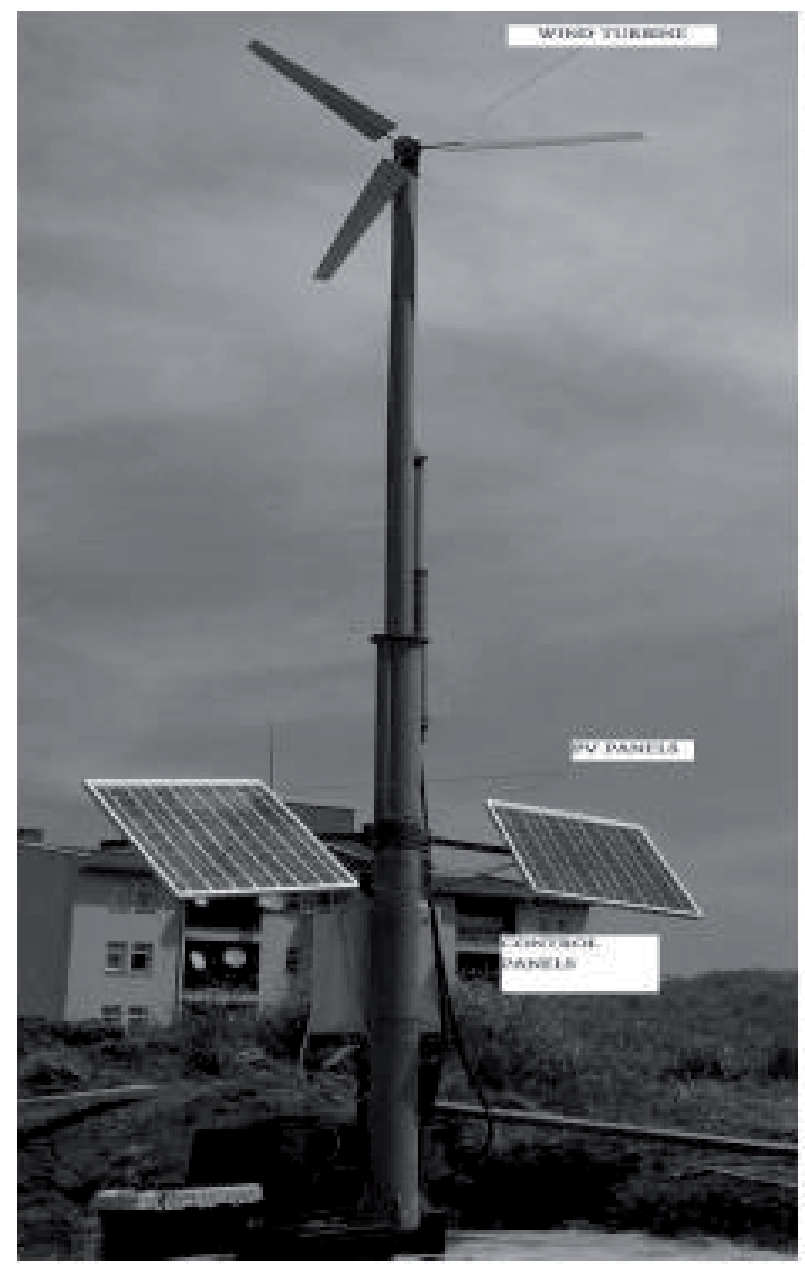

Figure 10.

Image of the designed hybrid system.

wind hybrid system. Simulation results show the outstanding effectiveness of the SA algorithm. The optimization study conducted [48] focuses on off-grid hybrid PV-Wind using different battery technologies based on genetic algorithm (GA) was successfully implemented.

Simulation based optimized design has been proposed for a PV/wind hybrid energy conversion system with battery storage under different load and auxiliary energy conditions was developed [21]. The simulation model of the system is implemented in ARENA 12.0, commercial simulation software, and is optimized using the Opt Quest tool in this software. Consequently, the optimum sizes of PV, wind turbine and battery capacity are attained under various auxiliary energy unit costs and two different loads. The best possible results are verified using loss of load probability (LLP) and autonomy analysis. And the financial commitment costs are examined how they are shared among those four energy sources at the optimum points.

Simulated annealing (SA) algorithm for optimizing size of a PV/wind integrated hybrid energy system with battery storage was reported [49]. The suggested technique is a heuristic strategy which utilizes a stochastic gradient search for the global optimization. The objective function is the minimization of the hybrid energy system total price. And the selection parameters are PV size, wind turbine 
rotor swept area and the battery capacity. The best possible result acquired by the SA algorithm when compared with other study's result. Therefore, it is actually coming up with that the SA algorithm provides much better result compared to Response Surface Methodology (RSM). The research study is realized for a campus area in Turkey.

Sizing optimization of off-grid PV-Wind using iterative approach was used in [7]. The proposed model takes into consideration the sub-models of the hybrid system, the Deficiency of Power Supply Probability (DPSP) and the Levelized Unit Electricity Cost (LUEC).

\subsection{Techno-economic optimization}

In this perspective, several techno-economic optimization approaches for hybrid systems sizing have been revealed in the literature. Iterative methodology has been applied to optimize the capacity sizes of various stand-alone PV/wind/diesel/ battery hybrid system parts for zero load energy shortfall [50]. The high price of renewable energy systems has brought to slow usage in many countries. Hence, Neuro-Fuzzy method was used for techno-economical of PV-Wind system. The optimization method used is Adaptive Neuro-Fuzzy Inference System (ANFIS), which is successfully applied to model the PV and wind sources. Comparison was made with hybrid optimization model for electric renewables (HOMER) and hybrid optimization by genetic algorithms (HOGA) software and the results prove an accuracy of $96 \%$ for PV and wind [51]. The optimized system is simulated in PSCAD/EMTDC and the results show that low excess energy is realized. In this work [10], the technical-economic optimization research of a stand-alone hybrid $\mathrm{PV} /$ wind system (HPWS) in Corsica Island is introduced. Consequently, the main purpose of the research is to calculate the acceptable dimensions of a stand-alone HPWS that ensure the energy independence of the typical rural consumer with the lowest levelized cost of energy (LCE).

It is acknowledged that solar energy and wind energy are two of the most feasible renewable energy resources on the globe, The work of [8] highly recommend an ideal design model for designing hybrid solar-wind systems making use of battery banks for determining the system optimum options and guaranteeing that the annualized cost of the systems is reduced while fulfilling the customized needed loss of power supply probability (LPSP). The five selection parameters involved in the optimization method are the PV module number, PV module slope angle, wind turbine number, wind turbine installation height and battery capacity. The offered technique is used to design a hybrid system to supply power for a telecommunication relay station along Southeast Coast of China.

In a related design techno-feasibility of hybrid PV-Wind was employed for a household in China, using HOMER simulation software. The design PV-Wind ration is $72: 28$, based on the detailed feasibility study conducted in the study areas. Another similar design in Indonesia [52] focuses on onshore remote areas. HOMER software is used to perform the techno-economic feasibility of the PV/wind hybrid system. The final results also display that a wind turbine and battery are the most significant elements of the PV/wind hybrid system to fulfill requirement loads at night hours. Considering that both of these components give the best advantages to system costs, it is very important to select their utmost sizes to reduce the costs, but by considering that no loads are unmet. Object oriented programming was applied to optimize hybrid PV-Wind system in a study conducted by Belmili et al. [53]. Detailed mathematical model was developed together with optimal algorithm for sizing and techno-economic analysis to identify the system that could ensure an effective energy supply with a most affordable financial commitment. 


\section{Conclusions}

This chapter presents detailed work conducted on hybrid system based on PV and Wind. The chapter systematically shows the different methodology used in the design, simulation, optimization and techno-economic aspects of PV-Wind Systems. Some design and application of the hybrid PV-Wind are discussed.

Hybrid renewable energy power system optimal design includes feasibility studies, model-based design, simulation and integration of several hybrid renewable energy resources, energy conditioner, and hybrid energy storage system and hybrid controller for automation to achieve power supply reliability. A hybrid renewable energy system (HRES) technology for reliable power supply has challenges in the design process. Thus, hybrid energy harvester, energy conditioner, energy storage and controller feasibilities, selection and unit sizing, and system configurations are necessary procedures to be carried out. Hybrid energy system components for power, reliability applications related to hybrid energy systems, power system has been reviewed above. In order to highlight the merits of the optimal design of hybrid energy system with a promising sustainable solution for power supply reliability.

The solar photovoltaic flat plate has of enormous adaptable models with the adequate alternative energy potential that could possibly replace conventional fossil fuel system. Application of the best possible of hybrid SPV plate/PEMFC resources, boost maximum power point tracking, integrated multi-level inverter and the hybrid PEMFC-Na_S battery storage, and micro hydropower system yields reliable electricity supply for rural and remote areas.

Hybrid renewable energy power system can offer socio-economic return when enough power is available in rural areas as business activities is going to be established as the communities do some corn/wood mills, small scale industrial ventures to engage more youth in entrepreneurship.

\section{Acknowledgements}

The authors would like to acknowledge with gratitude the support of Universiti Malaysia Sarawak (UNIMAS) under Postdoctoral Fellowship PD/16/016. Similar thanks go to Kano University of Science and Technology, (KUST) Wudil under UNIMAS-KUST partnership.

\section{Conflict of interest}

The authors have declared that no conflict of interest with regards to the publication of this chapter. 
A Review of Hybrid Renewable Energy Systems Based on Wind and Solar Energy: Modeling... DOI: http://dx.doi.org/10.5772/intechopen. 85838

\section{Author details}

Salisu Muhammad Lawan ${ }^{1 *}$ and Wan Azlan Wan Zainal Abidin ${ }^{2}$

1 Kano University of Science and Technology, Wudil Kano State, Nigeria

2 Universiti Malaysia Sarawak, Sarawak, Malaysia

*Address all correspondence to: mlsalisu@unimas.my

\section{IntechOpen}

(C) 2020 The Author(s). Licensee IntechOpen. This chapter is distributed under the terms of the Creative Commons Attribution License (http://creativecommons.org/licenses/ by/3.0), which permits unrestricted use, distribution, and reproduction in any medium, provided the original work is properly cited. (cc) BY 


\section{References}

[1] Lawan SM, Azlan W, Abidin WZ, Lawan M. Wind energy assessment and mapping using terrain nonlinear autoregressive neural network (TNARX) and wind station data. Cogent Engineering. 2018;5:1452594

[2] Safari B. Modeling wind speed and wind power distributions in Rwanda. Renewable and Sustainable Energy Reviews. 2011;15(2):925-935

[3] Güney MS, Kaygusuz K. Hydrokinetic energy conversion systems: A technology status review. Renewable and Sustainable Energy Reviews. 2010;14(9):2996-3004

[4] Boubekri N, Shaikh V. Machining using minimum quantity lubrication: A technology for sustainability. International Journal of Applied Science and Technology. 2012;2(1):111-115

[5] Ferdous RM, Reza AW, Siddiqui MF. Renewable energy harvesting for wireless sensors using passive RFID tag technology: A review. Renewable and Sustainable Energy Reviews.

2016;58:1114-1128

[6] Hong YY, Lian RC. Optimal sizing of hybrid wind/PV/diesel generation in a stand-alone power system using markov-based genetic algorithm. IEEE Transactions on Power Delivery. 2012;27(2):640-647

[7] Kaabeche A, Belhamel M, Ibtiouen R. Sizing optimization of gridindependent hybrid photovoltaic/ wind power generation system. Energy. 2011;36(2):1214-1222

[8] Ma T, Yang H, Lu L. A feasibility study of a stand-alone hybrid solarwind-battery system for a remote island. Applied Energy. 2014;121:149-158

[9] Lu L, Yang H, Burnett J. Investigation on wind power potential on Hong Kong
islands-An analysis of wind power and wind turbine characteristics. Renewable Energy. 2002;27(1):1-12

[10] Diaf S, Notton G, Belhamel M, Haddadi M, Louche A. Design and techno-economical optimization for hybrid PV/wind system under various meteorological conditions. Applied Energy. 2008;85(10):968-987

[11] Rao NS. Design \& simulation of hybrid solar-Wind electric power system interface to grid system. 2013;1(4):1-10

[12] Mohammadi M, Hosseinian SH, Gharehpetian GB. Optimization of hybrid solar energy sources/wind turbine systems integrated to utility grids as microgrid (MG) under pool/bilateral/hybrid electricity market using PSO. Solar Energy. 2012;86(1):112-125

[13] Daut I, Irwanto M, Irwan YM, Gomesh N, Ahmad NS. Potential of solar radiation and wind speed for photovoltaic and wind power hybrid generation in Perlis, Northern Malaysia. 2011. pp. 6-7

[14] Ashourian MH, Cherati SM, Mohd Zin AA, Niknam N, Mokhtar AS, Anwari M. Optimal green energy management for island resorts in Malaysia. Renewable Energy. 2013;51:36-45

[15] Mat S, Othman MY, Zaharim A, Sopian K. Recent advances in energy \& environment: proceedings of the 7th WSEAS International Conference on energy \& environment (EE'09). Cambridge, UK: WSEAS; 24-26 February 2009. pp. 246-250

[16] Giraud F, Salameh ZM. Steadystate performance of a grid-connected rooftop hybrid wind-photovoltaic power system with battery storage. IEEE Transactions on Energy Conversion. Mar 2001;16(1):1-7 
[17] Republic of the Philippiines. The Law to Enhance Mobility of Disabled Persons. 1983. pp. 1-30

[18] Nema P, Rangnekar S, Nema RK. Pre-feasibility study of PV-solar/ wind hybrid energy system for GSM type mobile telephony base station in Central India. In: 2010 The 2nd International Conference on Computer and Automation Engineering (ICCAE). Vol. 5. 2010. pp. 152-156

[19] Bekele G. Feasibility study of solar-wind based standalone hybrid system for application in Ethiopia. World Renewable Energy Congress. 2011;3(0):826-833

[20] Al Badwawi R, Abusara M, Mallick T. A review of hybrid solar PV and wind energy system. Smart Science. 2015;3(3):127-138

[21] Ekren BY, Ekren O. Simulation based size optimization of a PV/wind hybrid energy conversion system with battery storage under various load and auxiliary energy conditions. Applied Energy. 2009;86(9):1387-1394

[22] Arabali A, Ghofrani M, EtezadiAmoli M, Fadali MS. Stochastic performance assessment and sizing for a hybrid power system of solar/wind/ energy storage. IEEE Transactions on Sustainable Energy. 2014;5(2):363-371

[23] Hakimi SM, Moghaddas-Tafreshi SM. Optimal sizing of a stand-alone hybrid power system via particle swarm optimization for Kahnouj area in south-east of Iran. Renewable Energy. 2009;34(7):1855-1862

[24] Nfah EM, Ngundam JM, Tchinda R. Modelling of solar/diesel/battery hybrid power systems for far-North Cameroon. Renewable Energy. 2007;32(5):832-844

[25] Khan MJ, Iqbal MT. Pre-feasibility study of stand-alone hybrid energy systems for applications in Newfoundland. Renewable Energy. 2005;30(6):835-854

[26] Nema P, Nema RK, Rangnekar S. Minimization of green house gases emission by using hybrid energy system for telephony base station site application. Renewable and Sustainable Energy Reviews. 2010;14(6):1635-1639

[27] Liu LQ, Wang ZX. The development and application practice of wind-solar energy hybrid generation systems in China. Renewable and Sustainable Energy Reviews. 2009;13(6-7):1504-1512

[28] Bartlett DJ. Comparison of 15-month motor and 18-month neurological outcomes of term infants with and without motor delays at 10-months-of-age. Physical \& Occupational Therapy in Pediatrics. 2000;19(3-4):61

[29] Zhou T, Sun W. Optimization of battery-supercapacitor hybrid energy storage station in wind/solar generation system. IEEE Transactions on Sustainable Energy. 2014;5(2):408-415

[30] Daniel SA, Ammasai Gounden N. A novel hybrid isolated generating system based on PV fed inverter-assisted wind-driven induction generators. IEEE Transactions on Energy Conversion. 2004;19(2):416-422

[31] Harini M, Ramaprabha R, Mathur BL. Modeling of grid connected hybrid wind/PV generation system using Matlab. Journal of Engineering and Applied Science. 2014;7(9):1157-1161

[32] Dihrab SS, Sopian K. Electricity generation of hybrid PV/wind systems in Iraq. Renewable Energy. 2010;35(6):1303-1307

[33] Rehman S, Mahbub Alam M, Meyer JP, Al-Hadhrami LM. Feasibility study of a wind-PV-diesel hybrid power 
system for a village. Renewable Energy. 2012;38(1):258-268

[34] Sopian K, Ibrahim MZ, Wan Daud WR, Othman MY, Yatim B, Amin N. Performance of a PV-wind hybrid system for hydrogen production. Renewable Energy. 2009;34(8):1973-1978

[35] Mikati M, Santos M, Armenta C. Electric grid dependence on the configuration of a small-scale wind and solar power hybrid system. Renewable Energy. 2013;57:587-593

[36] Vick BD, Clark RN, Ling J, Ling S. Remote solar, wind, and hybrid solar/ wind energy systems for purifying water. Journal of Solar Energy Engineering. 2003;125(1):107

[37] Jacob T, Arun S. Modeling of hybrid wind and photovoltaic energy system using a new converter topology. Electrical and Electronics Engineering: An International Journal. 2012;1:1-13

[38] Moubayed N, El-Ali A, Outbib R. Control of an hybrid solar-wind system with acid battery for storage. WSEAS Transactions on Power Systems. 2009;4(9):307-318

[39] Kim SK, Kim ES, Ahn JB. Modeling and control of a grid-connected wind/ PV hybrid generation system. In: 2005/2006 IEEE/PES Transmission and Distribution Conference and Exhibition. Vol. 1. 2006. pp. 1202-1207

[40] Fontes N, Roque A, Maia J. Micro generation-Solar and wind hybrid system. In: 2008 5th International Conference on the European Electricity Market. 2008

[41] Soysal OA, Soysal HS. A residential example of hybrid wind-solar energy system: WISE. In: 2008 IEEE Power and Energy Society General MeetingConversion and Delivery of Electrical Energy in the 21st Century; 2008. pp. 1-5
[42] Ahmed NA, Miyatake M. A stand-alone hybrid generation system combining solar photovoltaic and wind turbine with simple maximum power point tracking control. In: 2006 CES/IEEE 5th International Power Electronics and Motion Control Conference. Vol. 1. 2007. pp. 242-248

[43] Mannah MA, Koubayssi A, Haddad A, Salami B. International journal of engineering research and applications (IJERA). Vol. 7, no. 2. IJERA

[44] Ambia MN, Islam MK, Shoeb MA, Nasimul NI, Mohsin ASM. An analysis \& design on micro generation of a domestic solar-wind hybrid energy system for rural \& remote areas-Perspective Bangladesh. In: 2010 2nd International Conference on Mechanical and Electronics Engineering. Vol. 2, No. Icmee. 2010. pp. $107-110$

[45] Koutroulis E, Kolokotsa D, Potirakis A, Kalaitzakis K. Methodology for optimal sizing of stand-alone photovoltaic/wind-generator systems using genetic algorithms. Solar Energy. 2006;80(9):1072-1088

[46] Hocaoğlu FO, Gerek ÖN, Kurban M. A novel hybrid (wind-photovoltaic) system sizing procedure. Solar Energy. 2009;83(11):2019-2028

[47] Askarzadeh A. A discrete chaotic harmony search-based simulated annealing algorithm for optimum design of PV/wind hybrid system. Solar Energy. 2013;97:93-101

[48] Merei G, Berger C, Sauer DU. Optimization of an off-grid hybrid PV-wind-diesel system with different battery technologies using genetic algorithm. Solar Energy. 2013;97:460-473

[49] Ekren O, Ekren BY. Size optimization of a PV/wind hybrid energy conversion system with battery 
A Review of Hybrid Renewable Energy Systems Based on Wind and Solar Energy: Modeling... DOI: http://dx.doi.org/10.5772/intechopen. 85838

storage using simulated annealing. Applied Energy. 2010;87(2):592-598

[50] Kaabeche A, Belhamel M, Ibtiouen R. Sizing optimization of grid-independent hybrid photovoltaic/ wind power generation system. Energy. 2011;36(2):1214-1222

[51] Rajkumar RK, Ramachandaramurthy VK, Yong BL, Chia DB. Techno-economical optimization of hybrid PV/wind/battery system using Neuro-fuzzy. Energy. 2011;36(8):5148-5153

[52] Hiendro A, Kurnianto R, Rajagukguk M, Simanjuntak YM, Junaidi. Techno-economic analysis of photovoltaic/wind hybrid system for onshore/remote area in Indonesia. Energy. 2013;59:652-657

[53] Belmili H, Haddadi M, Bacha S, Almi MF, Bendib B. Sizing standalone photovoltaic-wind hybrid system: Techno-economic analysis and optimization. Renewable and Sustainable Energy Reviews. 2014;30:821-832 



\title{
Grid-Connected Distributed Wind-Photovoltaic Energy Management: A Review
}

\author{
Sasmita Behera and Bibhuti Bhusan Pati
}

\begin{abstract}
Energy management comprises of the planning, operation and control of both energy production and its demand. The wind energy availability is site-specific, time-dependent and nondispatchable. As the use of electricity is growing and conventional sources are depleting, the major renewable sources, like wind and photovoltaic (PV), have increased their share in the generation mix. The best possible resource utilization, having a track of load and renewable resource forecast, assures significant reduction of the net cost of the operation. Modular hybrid energy systems with some storage as back up near load center change the scenario of unidirectional power flow to bidirectional with the distributed generation. The performance of such systems can be enhanced by the accomplishment of advanced control schemes in a centralized system controller or distributed control. In gridconnected mode, these can support the grid to tackle power quality issues, which optimize the use of the renewable resource. The chapter aims to bring recent trends with changing requirements due to distributed generation (DG), summarizing the research works done in the last 10 years with some vision of future trends.
\end{abstract}

Keywords: distributed generation, energy management, forecast, control, grid-connected

\section{Introduction}

A renewable hybrid energy system comprises of a couple of energy sources, a power conditioning device, a controller and sometimes with an energy storage system. When such renewable energy sources (RES) are integrated to the grid, variable output due to the stochastic nature of input may lead to instability and power quality issues [1]. In this changing scenario, micro-grids (MGs) have come up as a solution to maintain power supply in small scale as an autonomous entity in the event of grid failure. It has complementing resources or different DG sources in combination with storage with power electronic interface. Distributed energy resource (DER) can be either a distributed generator or distributed energy storage. Under its spectrum, it can be PV, wind, heat pumps, combined heat and power (CHP) generation, energy storage (ES), fuel cells (FCs), electric vehicles (EVs), energy efficiency (EE) and demand response (DR). The behavior of the resources, such as EE, DR, heat pumps, and EVs, is user dependent. Further, the PV source has no inertia. So ES and FCs can provide more reliability and flexibility to the grid 
if operated in a manner coincident with grid needs that respect storage limitations. These DGs have made the grid more resilient, efficient, environment-friendly, flexible, less vulnerable, easier to control, immune to issues at some other location, slow gradual capital investment, integrating to grid with minimal disturbance to existing loads during commencing. Participation of DERs in operation is profitable in respect of load shifting without grid up-gradation curtails peak demand, grid support by storage responding to demand thereby improving frequency response reducing spinning reserve. EVs and MGs can provide ancillary services. Under normal operation of the grid, varying capabilities of the DERs support voltage and reactive power whereas under fault voltage and frequency ride through capability is expected. Under such fault, the inverter must respond as per requirement. With the coordination of inverter-based resources in a group, it is possible that the DERs counteract to grid contingencies such as voltage and frequency deviations, and assist in fast recovery. So they are termed virtual inertia. But, at the same time, some issues are of concern and have drawn the attention of researchers. They are mainly due to stochastic nature such as load following, power vs. energy profile in storage, stability, reliability, cost, control architecture, autonomous control, power quality issues and grid interconnection. Considering these issues, in [2] the feasibility study, the unit commitment for reliable power supply and modeling of energy systems of PV, wind and diesel generator are focused. In the past decade, more significant development has taken place with various combinations of sources and storage. Optimization in all respect of wind energy for grid integration has been thoroughly reviewed [3] and observed to have good success. The control topology and the objectives have also changed in recent years. In addition to other reviews, control aspects and reliability issues with such sources are discussed [4]. The application of evolutionary technique and game theory in hybrid renewable energy is also presented. The chapter revisits the changing requirements due to DG, summarizing the research works done in the last 10 years with some vision of future trends.

\section{Regulatory, project finance and technical perspective}

The renewable electricity demand is predicted to add up 20\% more within the next 5 years. They can have the quickest development within the power sector, providing nearly $1 / 3$ rd of the requirement in 2023 [5]. Further, there is forecast to exceed $70 \%$ of world electricity produced, primarily by PV and followed by wind, hydropower, and bio-energy. Hydropower remains the biggest such supply, meeting $16 \%$ of the world electricity demand by 2023, followed by wind (6\%), PV (4\%), and bio-energy (3\%). Energy storage for grid applications lacks a sufficient regulatory history.

Whereas active regulation of voltage was not permitted and the DERs had to trip on abnormal voltage or frequency, participation in voltage and frequency control was desirable due to a gradual increase of the percentage of DER in power system. This was resolved in 2003. The first amendment to this came after a decade (11 years) but the second one came just after 4 years of first [6]. This comes in line with the steeper increase of DER penetration than the previous decade. As the DER are geographically dispersed, the communication interface between DER and the main grid and in between the DERs has been an additional demand of the hour for smooth and reliable coordinated control.

Some of the distribution grid safety demands are (1) short trip times, (2) ridethrough with momentary cessation (3) voltage rise concerns (4) protection coordination (5) islanding concerns for the safety of workers. Bulk system reliability demands (1) long trip times (2) ride-through without momentary cessation (3) reactive power support. 
Increasing penetration of unconventional generation to grid is reducing system inertia which can degrade system frequency stability. So, active power output is modulated in response to frequency deviation (Default droop 0.05 p.u. frequency for 1 p.u. active power change).

Voltage benchmarks standard for voltage fluctuations is within $\pm 5 \%$ at customer end. As a DER exports active power, voltage rises and the profile is disturbed and quality is compromised.

Current grid standards massively need that low-power KW range single-phase PV systems supply at unity power factor with maximum power point tracking (MPPT), and detect fault and island from the grid in such situation [7]. However loss of these generations under grid faults gives rise to voltage flickers, power outages, and an unstable system. So grid code amendments for increased entry of PV systems in the distribution grid are expected. The standards have undergone a significant review for low-tension interconnection in many countries. Also, reactive power can be supported either by changing the tap setting of the transformer or by the PV inverters with advanced control strategies to maintain the grid voltage.

Investments in RES for utility are normally assessed from regulatory, project finance, and technical perspectives. The regulatory requirement is satisfied by utility compliance as well as reduction of the associated cost. The budget estimate looks at the investment and benefits of the particular project. The technical assessment deeply goes through the safety concern of the specific technology involved and its operation. Besides these project specific assessments for RES, physical benefits of transmission and storage and the effect in the integrated picture of the grid is also important. It is therefore always recommended to go for an integrated approach for full exploitation of renewable generation and electricity storage with respect to transmission and distribution [8]. And this in line with the state utility cannot be undermined also. It is further recommended at all levels to increase its research and development $(\mathrm{R} \& \mathrm{D})$ in basic electrochemistry to find out the materials and discover the electrochemical technology suitable for use in grid. Department of energy, of concerned countries, have been leading the R\&D to deal with reliability, resilience, cyber security, and affordability issues from the grid modernization perspective.

\section{Weather-dependent variability of renewable resources}

The wind energy and PV are expected to have a lion's share in the prospect of the power utility. So, the future energy source is pivoted on the in-depth realization of their variability. Resource variability is a multi-faceted notion expressed by a range of distinctive characteristics. Simultaneously, research to date tells that there is restricted knowledge about the variability of the future power system. The variable attribute of climatic fluctuations is the reason of inconsistency of the RES and creates uncertainty in the energy production on the range of seconds, hours and days even. It is estimated that clouds limit up to $70 \%$ of daylight hour solar energy potential. Grid sometimes deals with aggregation over massive areas and this mitigates the variability of every single RES.

Presently a large variation is tackled via switching in fast-acting conventional sources depending on the climate forecasts on a minute-by-minute and hourly basis. Such variability can additionally be taken care by setting up large scale storage on the grid or; by the long-distance transmission of RE linking to larger pools of such generations in order to equalize regional surplus or shortfall nearby in future. Graabak et al. [9] have addressed the variability characteristics such as: (i) Distribution long term, (ii) distribution short term, (iii) step changes, (iv) autocorrelation, (v) spatialcorrelation, (vi) cross-correlation and (vi) predictable pattern. 
Distribution can be short-term (minutes, less than $1 \mathrm{~h}$ ) or long-term ( $1 \mathrm{~h}$ or more). These terminologies carry their own implication.

Many such related papers refer to "step changes" as a variability characteristic. These are the alteration in the available resource that takes place in small time steps of minutes to some hours. Another variable characteristic is autocorrelation [10] which figures out the statistical relation among values of the same parameter in a series. The relationship of wind speed information between different locations and the corresponding relationship of solar irradiance for different locations are under study by several projects. This spatial correlation is perceived as one of the instrument to gauge variability characteristics. Wind and solar sources may also show one kind of diurnal and seasonal trends.

Power from sun, wind, and ocean additionally exhibit predictable seasonal patterns recognized as a distinguishing variability characteristic. Pattern forecast for this trend of wind and sun is complicated, and it is a subject matter taken up in many papers. In a precise study, Tande et al. [11] have viewed reanalysis data set for illustrating of wind variability characteristics. With information of a temporal resolution of $6 \mathrm{~h}$ and a spatial resolution of $2.5^{\circ}$ in each latitude and longitude, a two-dimensional linear interpolation of neighboring locations is utilized to get wind speeds at the chosen sites. Both offshore and onshore information can be dealt with in this way for explaining the variability. It is apparent that entry of offshore wind generation and its variability will noticeably affect the grid.

In the study performed by Wiemken et al. [12] record from 1995 extracted from 100 monitored PV systems (rooftop plants $1-5 \mathrm{~kW}$ ) with a 5 min time resolution ensembled for $243 \mathrm{~kW}$ (grid connected) is used. A model is developed taking onshore wind and PV energy generation for the period 2001-2011 across 27 nations in Europe. The data is taken from NASA for hourly values of wind speed and solar irradiance documented at a spatial resolution of $0.5^{\circ} \mathrm{E} / \mathrm{W}$ and $0.66^{\circ} \mathrm{N} / \mathrm{S}$. The generation from wind and PV translated from the climatic record were later on combined to structure regional or nation-specific datasets. The model first considered PV and wind sources to contribute half of the energy supply of total requirement. Further $\mathrm{PV}$ share in the wind/PV proportions of $0,20,40$ and $60 \%$ are investigated.

\section{Power generation forecasts}

Contribution of wind energy has been the largest share out of the renewable energies and expects growth further. For responsible and sustainable growth of wind energy industry, reliability, robustness and stability are important factors. As wind energy integration to the grid is in MW scale, in future it may function as base load plant. So, the decision of economic load dispatch will largely be affected by proper forecasting of wind power. The objective is to improve accuracy in forecasting wind speed and power 1 day ahead so that it becomes reliable, which will be a benefit to the load dispatch centers as well as installation of additional wind turbines onshore and offshore.

Wind forecasting has been taken up in literature by various researchers. The forecasting for power may be very short term (within $2.5 \mathrm{~s}$ ), short term (10 min to $1 \mathrm{~h}$ ), long term (15 min to $3 \mathrm{~h}$ ) or a day ahead ( $24 \mathrm{~h}$ ). Forecasting wind speed is an important factor, based on which planning of new wind farm depends. Specifically for offshore wind farms, the safety requirement has less advanced. As wind speed prediction and power prediction takes time for computation and error in forecasting wind power 1 day ahead is more compared to the short term, there is a need for improvement. Research has shown good result from the hybrid method. The researchers are oriented to make wind power predictable. When the wind is 
predictable, it becomes reliable, which will be a benefit to the load dispatch centers for economic load dispatch as well as the installation of additional wind turbines onshore and offshore.

Going through the available tools and the accuracy, the methods/prediction models are broadly divided into physical, statistical and artificial intelligence based methods [13]. Out of various statistical methods such as curve fitting, statistical approximation autoregressive integrated moving average (ARIMA), seasonal ARIMA, extrapolation with periodic function, methods of finding probability density functions (PDF) have been evaluated by the coefficient of determination [14]. Different software models have been developed such as WPMS, WPPT, Prediktor, ARMINES, Previento, Zephyr, AWPPS, Ewind, ANEMOS and adopted in different countries [15]. Some of them are hybrid methods. Prediction of offshore extreme wind is important for the protection of offshore wind system so that such sites can be avoided during planning. Method of independent storms (MIS) stands better as compared to the other three in the study by An et al. [16]. In another work, the extreme wind has been estimated by the combination of swarm optimization with the traditional methods which added improvement [17]. The available software has their limitations up to how many meteorological data required, precision in numerical weather prediction (NWP), different accuracy indices for short and long term prediction etc. Intelligent techniques such as Artificial Neural Network (ANN) [18], Fuzzy, Support Vector Machine (SVM), Wavelet, Hilbert-Huang transform, data mining techniques [19], swarm optimization combining the statistical methods of time series prediction with improvement in nonlinear node functions and training algorithms have given good results as compared to statistical/any method alone $[19,20]$. Combination of Fuzzy and ANN take less prediction time thus gives faster result [21]. It has been remarked that grouping wind farms for wind forecasting can give better result [19]. Instead of predicting the wind speed exactly, prediction into lower and upper bounds method (LUBE) [22] in prediction interval with defined confidence level gives better result in performance indices. Wind speed has been estimated by RBF (radial basis function) neural network and wind turbine has been appropriately controlled for maximization of wind power [23]. "Anti-phase correlation" of wind speed and solar radiation has been found after wavelet analysis, implying that wind and solar energy can complement each other in generating electricity [24].

Smart grid performs also with penetration of PV and has to consolidate its performance figures in the presence of variability. Many researchers report on the novel hybrid intelligent algorithm for PV forecasting taking its fluctuating behavior. In this regard, wavelet transforms (WT), stochastic learning, remote sensing method and fuzzy ARTMAP (FA) network. Forecasting accurately improves system efficiency also.

As numerical prediction depends on weather data, which is provided by sensors, reduction of dependence on sensors for wind speed, rather estimation method of wind speed for sensor-less control is the need.

Different capacity of battery, wind, PV is considered to check which proportion of each component is economical for a specific location Dhahran in Saudi Arabia taking historical weather data during the demand of different months in a year for the wind-PV hybrid power system (WPVHPS) [25]. The addition of wind generation is more economical than PV. The addition of more battery can reduce the diesel generation and time of use.

Prediction is vital for energy management [26]. The energy management functions in a wind-battery system are to (1) charge the battery from wind (2) supply the load from wind power (3) trade the wind/battery power to the grid (4) buy power from grid and store in the battery or supply it to the load; and (5) supply the local load from battery. The day ahead electricity rate and wind energy are 
forecasted through Wavelet-ARMA of time series breaking it into smooth subseries. The state of charge (SOC) of battery is predicted in a longer time horizon. In another case study of Turkey [27], based on 15 years of data of global solar radiation distribution, no relationship between the distribution of annual time lapse and solar energy and solar radiation intensity are established.

The solar and wind energy potential are surveyed for five sites in Corsica [28]. From this study, two sites with the desirable trait are chosen and the sizing and the economics for an isolated hybrid PV/wind systems are compared. The trend is dependent on site-specific resource analysis. The sites with more wind potential have less cost of energy and more feasibility.

\section{Planning}

Planning wind PV hybrid power system (WPVHPS) involves a cost-effective design on priority. The various aspects that are optimally adjusted before commencing are size, fluctuation of load and generation. But, some design considerations such as tilt angle of PV panel and a hub height of wind turbine too have importance. Besides the priority objective, when the reliability of supply is seen, the optimum number of units plays an important role. The years of service life is also important in planning. Graphical construction and probabilistic approaches in combination with an optimization method are used for planning. Planning has become a multiobjective optimization with multi-dimension.

Yang et al. [29] optimally designed wind- solar-battery system for the minimal annualized cost satisfying the limit of loss of power supply probability (LPSP). The five factors such as number PV module, wind turbine and battery units; module inclination, and height of wind turbine have been optimized by genetic algorithm (GA). The result is indicative that the minimum number of wind turbine with some batteries and PV panels with the location-dependent tilt angle is a good solution.

After going through various traditional approaches for their suitability for wind-PV hybrid systems Sinha et al. [30] suggest for using a hybrid of multiple algorithms which can remove the shortcomings of a single method. Abbassi et al. [31] discuss the battery for energy storage which is slow but super-capacitor is fast in giving away the power to the peak load. The energy management is influenced by proper sizing of these storages. The statistical probability density functions are considered for wind speed and irradiation. Discrete Fourier transform (DFT) of the output power to different fast and slow components is done. Monte Carlo simulation (MCS) for different scenarios is very useful for confirming a design for such stochastic variations of generation and load. One contribution of the storage in such system is towards the frequency management. In a similar line, Arabali et al. [32] suggest a new strategy to meet the controllable heating, ventilation, and air conditioning (HVAC) load with a hybrid-RES and ES system. From recorded weather data and load stochastic model of the wind generation, PV generation, and load are developed by Fuzzy C-Means (FCM) clustering dividing data into 10 clusters to show seasonal variations. A multi-objective GA is employed to get the optimal size, cost, and availability DC micro-grid systems with PV and wind [33]. When planned with high-temporal resolution data increased control, improved export, availability of power and decreased variability than for hourly data set. The diesel generator is initially thought as an alternate supply once power fails because it is well transferrable, standard and has a high power-to-weight ratio [2]. When various DERs are integrated into the system, these can affect the voltage profile of the system and demands frequent tap change, but if the voltage is set based on one fixed point, there may be an overvoltage at another. During planning in addition to 
overall operational cost, the capacity of capacitor bank or power factor correction equipment and inverter control are also to be considered [34].

\section{Operation}

\subsection{Energy management}

Energy management system (EMS) is an integration of all the algorithms procedures and devices to control and reduce the usage and the cost of energy used to deliver the load with its specifications. In a critical review [35] it has been pointed out that, most of the EMS for RES is concerned with flow and control of power and efficient battery utilization for its durability. But, a full-fledged control approach is yet to be developed.

Wu et al. [36] proposed optimal scheduling of the PV system for saving the timeof-use (TOU) cost. Sichilalu et al. [37] focused on a net-zero-energy building by demand side management. The energy management of a grid-connected WPVHPS has been introduced in hardware [38]. In this paper, the hardware, communication and how to meet its requests and functions are emphasized. The system could manage both grid-connected mode and stand-alone mode. EMS for both standalone and grid-connected hybrid RES are reviewed by Olatomiwa et al. [39]. EMS based on linear programming, intelligent techniques and Fuzzy logic controllers is discussed for various combinations. In the study [40] an EMS for controlling end-user building loads, AC, light, ice storage discharge, with adequate solar rooftop PV systems in groups to absorb PEV penetration using practical charging situations are developed without delaying EV charging. The EMS is developed in [41] for a micro-grid with RES that checks net excess generation, battery power and SOC and takes the decision whether to charge/discharge the battery, reduce PV generation, shed load or increase generation of PV by MPPT to control load end voltage. Boukettaya et al. [42] developed a supervisory control in a MG with WPVHPS, a flywheel energy storage system (FESS). Reihani et al. [43] studied the EMS for a MW-range battery energy storage system (BESS) with actual grid data serving for peak load shaving, power smoothing, and voltage regulation of a distribution transformer.

A distributed algorithm that extracts renewable energy sources on high priority through monitor and prediction of generation and loads online is proposed in [44]. It works to reduce cost and improve system stability. In [45] reports a battery management system (BMS) based on physics-based models of lithium-ion (Li-ion) batteries and vanadium redox-flow (VRF) BESS. In [46] a VRF storage device for frequency regulation and peak-shaving tasks is demonstrated. Multiple BMSs are required in order to reach the desired capacities at grid level demand. A part of the (EMS) in order to achieve specific operational objectives is described in [47].

Gelazanskas et al. [48] review demand-side management (DSM) and DR, including incentives, non-critical load scheduling and peak shaving methods.

Vasiljevska et al. [49] demonstrated an EMS in a medium voltage (MV) network with several MGs by a hierarchical multi-level decentralized arrangement. A power management system (PMS) is proposed for a PV-battery-based hybrid DC/AC MGs for both grid-connected and islanded modes [50]. It balances the power flows, regulates bus voltage automatically under different operating circumstances.

\subsubsection{Centralized control}

A grid-connected hybrid system with battery is studied and tested for centralized control under three scenarios by [51]. The control strategy developed could 
maximize the utilization of the hybrid system. Centralized control requires fast communication and supercomputing to handle a large amount of data in a short time. This is less reliable due to single point attack risk. A new topology of WPVHPS is proposed by Singaravel et al. [52]. In this topology, the sources are connected together to the grid via only a single boost converter-inverter setup.

\subsubsection{Distributed control}

It is very suitable for grid-integrated renewable sources. Alagoz et al. [53] describe that DERs are gradually increasing count with each consumer turning into a prosumer. This can take the best out of it if there is a bidirectional interaction between DERs. A service-oriented infrastructure can be formed by a tree-like usermode network (UMN). In [54] the coordination control of a WPVHPS and a proton exchange membrane fuel cell (PEMFC) is studied. A grid-connected WPVHPS is proposed in [55]. The pitch control of the wind turbine uses radial basis function network-sliding mode (RBFNSM), and the MPPT of PV system uses general regression neural network (GRNN).

For control of the voltage and frequency at the point of common coupling firefly algorithm (FA) based proportional integral (PI), and PID controllers [56] are used. In [57] authors have shown that the modified adaptive accelerated particle swarm optimization (MAAPSO) proves better than PSO for PID and fractional order PID battery charge controller.

\subsubsection{Hybrid control}

This type is regarded as a combination of centralized and distributed control and is more versatile. Qi et al. [58] reviewed supervisory model predictive control (MPC) and developed it in distributed architecture taking two spatially distributed wind and PV subsystems each with storage, in a DC power grid, with a local load connected. For a WPVHPS with fuel cell, [59] a direct control scheme in a hybrid $\mathrm{AC} / \mathrm{DC}$ structure is developed that deploys a harmonic virtual impedance loop and compensates voltage.

\subsubsection{Control communication}

Dynamic interaction between transmission and distribution systems caused due to transformations in power systems make control vulnerable. This is also happening in case of integration of renewable power plants to grid. Vision for perfect grid management can never undermine the importance of control communication. If the output of a renewable energy power plant is greater than $10 \%$ of the line capacity, temporary unavailability [60] can adversely affect power grid stability, so demands a communication. It is important to develop an intelligent, self-adaptive, dynamic and open system. So, a multi agent system (MAS) is proposed [61] to handle the energy management of the hybrid PV-wind generation system in which each agent with a RES reacts intelligently to changes.

For the energy control in a distributed manner, energy routers can serve dynamically the energy distribution in the grid, where the whole structure can be termed as energy Internet [62]. Cao et al. [63] discuss in detail energy internet called as version 2.0 of smart grids that has the two-way flow of information and power. More openness and peer-to-peer communication are introduced. This network can balance power with more interaction and options. Anticipatory or predictive control is possible based on information to anticipate future states and appropriate decision-making for timely action. 
For peak load and outage, a building integrated PV (BIPV) mainly for selffeeding of buildings equipped with PV array and storage is studied in a DC MG [64]. Hierarchical control is designed by Petri nets (PNs) interface for a 4-layered EMS that regards the grid availability and user's commands. The layers are humanmachine interface (HMI), prediction, cost management, and operation.

\subsection{Power quality issues}

The power quality is also an issue with the WPVHPS integrated. This section briefly indicates the power quality problems in grid integration. A well-written review has been brought by [65] for problems and solutions so far concerned with such system in grid-connected condition. Voltage and frequency fluctuation and harmonics are major power quality issues with a severe effect on the weak grid. Appropriate design and advanced fast control can solve it. Filters, control of PWM inverter, and droop control can be a solution to it. Kabalci et al. [66] discuss a threephase inverter control scheme to limit total harmonic distortion within standards.

\section{Case study in Indian scenario}

According to the International Energy Agency, investment in renewables in India exceeded that for fossil fuel-based power generation in 2017. In India, the grid-interactive PV-wind generation of 688.42 MW is added in 2018-2019 with a cumulative of $64.5 \mathrm{GW}$ till March 2019 [67]. Till the end of the financial year 2017-2018 the total RE installed was $70 \mathrm{GW}$ whereas it is $79 \mathrm{GW}$ at the end of the financial year 2018-2019. As displayed in Figure 1 the latest RE update has major contributors are PV (36.2\%) and wind (45.3\%). A $41 \mathrm{MW}$ (25 MW PV + $16 \mathrm{MW}$ wind) with storage is under construction in Andhra Pradesh, India. This pilot project will work on efficient grid management through real-time monitoring of ramps, peak shifting and matching of load and generation profiles. India targets $175 \mathrm{GW}$ of installed capacity from RES by the year 2022, which includes $100 \mathrm{GW}$ of PV and $60 \mathrm{GW}$ of wind. To this effect, India's Ministry of New \& Renewable Energy (MNRE) released the National Wind-Solar Hybrid Policy in May 2016. It is framed to support large grid-connected WPVHPS for optimal and efficient utilization of transmission infrastructure and land, reducing the variability in renewable power generation and achieving better grid stability. Superimposition of wind and solar
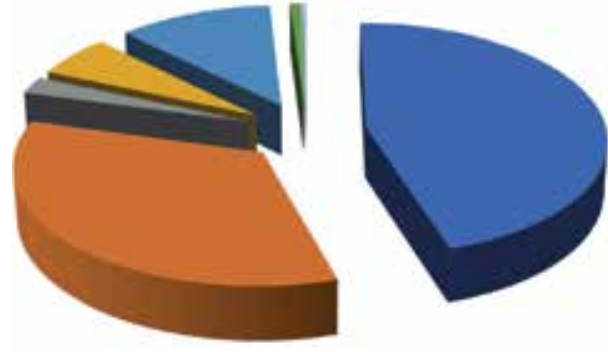

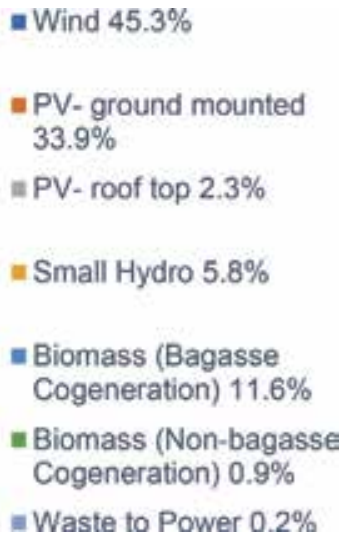

Figure 1.

Grid connected RE in India up to March 2019. 
resource can complement variability of both. As per the policy, a wind-PV plant is defined as a hybrid plant if one satisfies at least $1 / 4$ th of the rated power capacity of the other. Different configurations and use of technology for AC, DC integration with storage are encouraged with incentives as specified therein.

The Central Electricity Authority is empowered to frame the standards for connectivity and sharing of transmission lines, etc. for such systems. So in India case study in hardware with grid interaction are limited to academics. A case study of Barwani, [68] found that PV-wind-battery-DG hybrid system is the most optimal solution when cost and emission are the main targets. The work in [69] involves the development of the RE based hybrid system for electricity that can supply desired power continuously throughout the year irrespective of fluctuation of energy available from standalone systems. The energy assessment has been done using Homer simulation tool for developing a small solar-wind hybrid system, at National Institute of Engineering-Centre for Renewable Energy and Sustainable Technologies (NIECREST), Mysuru, India. The WPVHPS was fully charged during the day time and thereafter the performance was checked by connecting to $596 \mathrm{~W}$ load through the $1500 \mathrm{kVA}$ inverter and energy meter. The WPVHPS was able to supply energy for $3 \mathrm{~h}$ roughly in the evening.

\section{Storage for RES}

\subsection{The case of grid-level storage}

It should be noted that no ES technology claims high in all aspects. Each has its own limitation in performance when used for grid connection. System capacity, type of application and the cost of peak time electricity decide the storage capacity. A wide variety of such technology may be required to address the issues arising during grid connection.

In [70] an optimized sizing methodology for battery ES to cater peak shaving and ramp rate limiting in the power dispatch using bat algorithm and validated in a grid-connected WPVHPS to combat loss of power is presented. Five types of battery ES such as lead-acid (Pb-acid), Li-ion, flow batteries and sodium sulfur $(\mathrm{NaS})$ are tested in a comparative fashion. After examining storage technologies applied in four purposes such as frequency regulation, power smoothing for wind as base load plant, power smoothing for load following and peak shaving the authors [71] have arrived in a conclusion that the power accumulation capacity is vital for frequency regulation, whereas the energy capacity influences energy intensive applications like peak shaving. The transient stability of a DG-battery-super capacitor has been carried out by [72]. Korada et al. [73] have developed a three-level grid adaptive power management strategy (GA-PMS) in MG with RES—battery-supercapacitor to support grid.

A compressed air energy storage (CAES) and wind energy system is used in [74]. It is tried to time shift wind energy to maximize the daily revenue by stochastic dynamic programming (SDP) for forecasting generation and price.

With similar objective [75] has added an approximate dynamic programming (ADP) algorithm that shows the proficiency of designing near-optimal control policies for a large number of heterogeneous storage devices in a time-dependent environment with good accuracy at par with stochastic and dynamic models when demand-variability is additionally taken. The economic feasibility of a centralized CAES is more viable than the distributed wind turbines-CAES [76].

Koller et al. [77] shows the effect of the grid-connected 1 MW BESS on frequency reserves, peak clipping and islanded operation of a MG. Grid forming and grid following inverters for the variable RES is detailed in [78]. An online 
optimal operation [79] for BESS based on a mixed-integer-linear-program (MILP) is proposed over a rolling horizon window. After a detailed study of different batteries, Li-ion batteries of LFP-C type are suggested economical in long run for large capacities for stationary applications [80] with RES. The study on placement of storage by [81] indicates that the line-flow limits have a significant effect. Hybridizing PV-wind with micro-hydro power plants into a single mini-grid has been practically applied in Nepal [82] which has increased the reliability and meets the load in an environment-friendly way.

A study on grid-level FESS [83] showed that locating it at the transformer and higher levels in the grid will reduce its size by inherent power smoothing by the pool. The ability to exchange power with neighboring grids, load shifting and storage can deal with high penetration of renewable [84]. Peak shaving can be dealt with by gas powered generation and load leveling (flat profile) by coal-fired or battery or pump storage [85].

Williams et al. [86] put forth DSM by adding heat pumps and thermal storage to PV that adds on energy independence of the house.

\subsection{Battery energy storage technology and materials}

A battery ES with its own specific features can serve a particular usage when time, space, portability and size are some of the factors. This section reviews battery ES in view of the latest technologies, advantages, sizing, efficiency, price, and life cycle assessment.

The modern storage technologies with regard to wind power integration are discussed in [87] on which the planning rests upon. Output power smoothing operation by single or multiple ESS considering influencing factors as above is done. In the work [88], has cross-compared with the maturity level of the technology of storage.

Wide variety of storages have been detailed in [89] taking an in-depth study of the electrochemical properties of the BES. The energy capacity and the selfdischarge or capacity fade of BES systems affect the suitable storage duration. Study in [90] has shown that BES can go forward for ancillary service if its cost reduces. Palizban et al. [91] have pointed out that a hybrid of different energy storages can serve multiple purposes in a cost-effective way. In [92] the technical viability of Li-ion batteries for the inertial response (IR) in grids with ample contribution of wind power has been evaluated.

One particular BESS cannot suit for all the support services like short, medium as well as long term [93]. Only Li-ion can serve for short duration support. For distributed storage and medium duration support, $\mathrm{Pb}$-acid and $\mathrm{Li}$-ion batteries are most suitable. A lithium-antimony-lead ( $\mathrm{Li}-\mathrm{Sb}-\mathrm{Pb}$ ) liquid metal battery is proposed by [94] which has higher current density, longer cycle life and simpler manufacturing of large-scale stationary storage systems.

\subsection{Plug-in hybrid electric vehicles (PHEV)}

$\mathrm{RE}$ can be better absorbed if electric vehicle charging and discharging is done strategically. Conventional power supplies can be sent as needed to match demand and provide ancillary services for grid stability. Contribution to grid by RES is increasing although these sources are intermittent by nature. This is now an operational challenge to balance the intermittency of RES. Electric vehicles (EVs) offer a scope to manage demand and potentially mitigate the amount of curtailed energy by controlling when EVs are charged.

Different types of charger such as AC/DC, slow/fast are discussed in European standard by [95]. Integration of ESSs in EVs charging station has grown with AC 
but the DC system has higher energy efficiency with improvement up to $10 \%$ [96] with less number of conversion stages taking generation from RES. The important communication system, not less than the brain makes it all possible in a coordinated manner. It communicates with the smart metering system present on the MG and on the EV charging station, through Modbus on TCP/IP connection, using the internal LAN, and with the ES converters, through the CAN protocol.

When the market penetration of uncoordinated plug-in electric vehicles (PHEV) is studied by [97], it is encouraged for load control by smart charging. It can reduce the size of central storage devices.

Clement-Nyns et al. [98] have investigated charging and discharging of PHEV in a cooperative manner that helps the voltage control and reduces congestion.

The PHEVs as dynamically configurable dispersed storage can operate in vehicle-to-building (V2B). Based on the distinctive attribute of the battery, the benefits of using PHEVs as energy storage for DSM and outage management are deliberated by Pang et al. [99]. The faster-charging are yet to come up. The parking time can be utilized for charge or discharge mode when required.

\section{Long-distance transmission}

All countries emphasize on use of clean and alternate energy. As discussed in previous subsections, with the rapid development of RES fresh set of technological requirements pops up on the grid: the location of RE resources distant from load centers, and the power-variability. The characteristics and its control of the electricity grid need a modification to integrate RE [100]. At present many countries lack affordable storage facilities for renewable power. But on a positive note, the excess power is transmitted through the national grid by internal transmission lines. However, connectivity to the national grid should be even or balanced. The large scale intermittency demands to switch in fast-acting conventional reserves on the basis of climatic forecasts on short to long time frame; by setting up grid-scale storage or; by long-distance transmission of RE generation connecting to larger reserves for resources in order to equalize regional and local surplus or shortfall. This section discusses opportunities for renewable energy transmission over a long distance.

Long-distance transmission capacity is necessary to despatch a huge quantity of renewable power a thousand kilometer or more across the country. The construction of transmission tower is given low priority by historically low investment in transmission, community concern over the required right of way in more dense urban areas. Further many long transmission lines are aged and of inadequate capacity. Both remote solar PV and wind energy generation require "Green power Superhighways." HVDC transmission [100] and use of superconductors [100] are costly alternatives as RE itself cost more to the user. HVDC lines offer transient as well as short term voltage stability. Variability of the source can be well managed via an extensive and robust transmission line network. The transmission capacities based on power electronics devices starts to change the grid characteristics and control requirements. The key power electronic technology has a high impact on the power quality because of its fast control and sensitivity to fault and other abnormal conditions of the grid.

So, research is still going on HV superconducting cable for long-distance transmission of RE [101]. Anyway, in the present day of renewable energy, the grid has to serve national character. With more urbanization and industrialization, the reduction of carbon dioxide emission has been essential and requires long-distance delivery of renewable power [102]. Rooftop PV can reduce the need for long-distance transmission, but have a higher cost than wind or concentrating 
solar power, and with small but considerable esthetic sense. The gradual entry of big wind and solar generation demand huge spending of money in improving the capacity and efficiency of long-distance electricity transmission.

Many researchers feel till date that there is a growing gap between the grid system and control technologies and power electronics equipment design capability.

\section{Conclusion}

Renewable energy is environmentally, socio-ethically and economically sustainable compared with the dominant centralized and non-renewable energy generation systems. However, the techno-economic limitations for ever-growing renewables' share of power generation in the majority of the countries are alike.

The RES is not currently cost-competitive with base load coal-fired power, and geographically dispersed. However, it leads over a conventional generation in low emissions of air pollutants, free fuel, and a low gestation period.

Traditionally, the electric power system is not intended to handle RE generation and storage. But with the rapid growth in the alternate energy sector, the integration of the DE and RES into an electric power grid can be done in many ways along with power quality solution. The power electronic technology plays a significant part in the integration of RES into the electrical grid. They offer exclusive competence over conventional interconnection technologies. They further provide additional power quality and voltage/reactive power support.

This chapter describes the various aspects of grid interface for wind energy systems and solar PV systems and some other DERs for electrical system compatibility by reducing the effects on fault, and flexibility in functioning with various other DERs, while minimizing the interconnection costs.

Around 100 research papers in various problems of grid connection have been surveyed but claims in no way to be complete. This particular subject is definitely emerging in nature and attracts many researchers towards it.

It has been discussed that utility RE investments are typically evaluated from regulatory, project finance, and engineering viewpoints. The regulatory evaluation focuses on ensuring utility conformance to RES and that expenses are kept judiciously limited. From a finance perspective, the return on the investment within disjunctive limits of the funding and cash flows for a particular project is evaluated. The technical evaluation determines the engineering and operational safety of the project and the specific technologies deployed. While these approaches are essential for investors, utilities, regulators and ratepayers, they do not scope out the goodness that a RES can convey beyond the boundaries of a given project, such as the usefulness of transmission and storage and the organizational plus point of bringing an integrated grid.

Variability of RES occurs due to the nature of the climate. Thereby the uncertainty in the generation is affecting up to $70 \%$ of day time solar capacity due to passing clouds, and $100 \%$ of wind capacity on calm days, is much greater than the somewhat expected variations of a few per cent in demand that system operators handle. This has been discussed. It necessitates a more complicated voltage and frequency regulation. The larger the RE entrant, the more complicated (sometimes unattainable) is the management of this challenge.

Spatial aggregation of RES greatly lessens forecast errors, just as it lessens variability. This may be due to spatial smoothening effect. The forecast error rises further as the time range of the forecast is expanded. Forecasting techniques are improving constantly. But this requires better weather model and better data collection and processing. 
In contrast to the convention fossil fuel power sources, selecting a site to exploit certain RES has few or no degrees of freedom. In other words, RE such as wind and PV, are site-constrained. Transmission needs to be extended to these sources, not the other way around. Future distribution systems will contain MGs and hence it is necessary to understand the steady-state and transient operating conditions of such systems to appraise their effects on the present grid.

Control system is the key element for flexible operation, high efficiency and superior power quality in RE integration. In this regard, the control system fetches real-time states through local measures and via the communication, takes actions to attain the control objectives (for instance, maximum power extraction, output voltage and frequency regulation, reactive power compensation, etc.), and at last send commands to the actuators, usually power electronic converters. Challenges in control design and realization, energy management strategies, communication layout and protocols, and topologies for power electronics-based distributed RES are all addressed in brief here.

The value of energy storage by batteries in grid-level applications that guides both transmission and generation services to the grid. It mitigates the unpredictability of generation. It has been emphasized to conduct a review of the technological potential for a range of battery chemistries.

Lastly, it is reiterated again that because of some demerits and irrevocable externalities in conventional energy production, it has become essential to go for and uphold technologies and insist for RES. Power generation using RES should be enhanced in order to reduce the per unit price of generation.

\section{Author details}

Sasmita Behera ${ }^{1 *}$ and Bibhuti Bhusan Pati $^{2}$

1 Department of Electrical and Electronics Engineering, Veer Surendra Sai University of Technology, Burla, Odisha, India

2 Department of Electrical Engineering, Veer Surendra Sai University of Technology, Burla, Odisha, India

*Address all correspondence to: sasmitabehera2000m@gmail.com

IntechOpen

(C) 2019 The Author(s). Licensee IntechOpen. This chapter is distributed under the terms of the Creative Commons Attribution License (http://creativecommons.org/licenses/ by/3.0), which permits unrestricted use, distribution, and reproduction in any medium, provided the original work is properly cited. (cc) BY 


\section{References}

[1] Nema P, Nema RK,

Rangnekar S. A current and future state of art development of hybrid energy system using wind and PV-solar: A review. Renewable and Sustainable Energy Reviews. 2009;13(8):2096-2103. DOI: $10.1016 /$ j.rser.2008.10.006

[2] Kusakana K. Optimal scheduled power flow for distributed photovoltaic/ wind/diesel generators with battery storage system. IET Renewable Power Generation. 2015;9(8):916-924. DOI: 10.1049/iet-rpg.2015.0027

[3] Behera S, Sahoo S, Pati BB. A review on optimization algorithms and application to wind energy integration to grid. Renewable and Sustainable Energy Reviews. 2015;48:214-227. DOI: 10.1016/j.rser.2015.03.066

[4] Khare V, Nema S, Baredar P. Solarwind hybrid renewable energy system: A review. Renewable and Sustainable Energy Reviews. 2016;58:23-33. DOI: 10.1016/j.rser.2015.12.223

[5] Available from: https://www.iea.org/ renewables2018/

[6] Photovoltaics DG, Storage E. IEEE standard for interconnection and interoperability of distributed energy resources with associated electric power systems interfaces. IEEE Std. 2018:1547-2018. DOI: 10.1109/ ieeestd.2018.8332112

[7] Yang Y, Enjeti P, Blaabjerg F, Wang H. Wide-scale adoption of photovoltaic energy: Grid code modifications are explored in the distribution grid. IEEE Industry Applications Magazine. 2005;21(5):21-31. DOI: 10.1109/ mias.2014.2345837

[8] Zame KK, Brehm CA, Nitica AT, Richard CL, Schweitzer IIIGD. Smart grid and energy storage: Policy recommendations. Renewable and Sustainable Energy Reviews. 2018;82:1646-1654. DOI: 10.1016/j. rser.2017.07.011

[9] Graabak I, Korpås M. Variability characteristics of European wind and solar power resources-A review. Energies. 2016;9(449):1-31

[10] Coker P, Barlow J, Cockerill T, Shipworth D. Measuring significant variability characteristics: An assessment of the three UK renewables. Renewable Energy. 2013;53:111-120. DOI: 10.1016/j.renene.2012.11.013

[11] Tande JO, Korpås M, Warland L, Uhlen K, Van Hulle F. Impact of trade wind offshore wind power capacity scenarios on power flows in the European HV network. In: Proc. of the 7th Int. Workshop on Large-Scale Integration of Wind Power and on Transmission Networks for Offshore Wind Farms; May 2008; Madrid, Spain. 2008. pp. 26-27

[12] Wiemken E, Beyer HG, Heydenreich W, Kiefer K. Power characteristics of PV ensembles, experience from the combined power production of 100 grid connected PV systems distributed over the area of Germany. Solar Energy. 2001;70:513-518. DOI: 10.1016/ S0038-092X(00) 00146-8

[13] Lei M, Shiyan L, Chuanwen J, Hongling L, Yan Z. A review on the forecasting of wind speed and generated power. Renewable and Sustainable Energy Reviews. 2009;13(4):915-920. DOI: 10.1016/j. rser.2008.02.002

[14] Carta JA, Ramirez P, Velazquez S. A review of wind speed probability distributions used in wind energy analysis: Case studies in the Canary Islands. Renewable and Sustainable Energy Reviews. 2009;13(5):933-955. DOI: $10.1016 /$ j.rser.2008.05.005 
[15] Wang X, Guo P, Huang X. A review of wind power forecasting models. Energy Procedia. 2011;12:770-778. DOI: 10.1016/j.egypro.2011.10.103

[16] An Y, Pandey MD. A comparison of methods of extreme wind speed estimation. Journal of Wind Engineering and Industrial Aerodynamics. 2005;93(7):535-545. DOI: 10.1016/j.jweia.2005.05.003

[17] Wang J, Qin S, Jin S, Wu J. Estimation methods review and analysis of offshore extreme wind speeds and wind energy resources. Renewable and Sustainable Energy Reviews. 2015;42:26-42. DOI: 10.1016/j. rser.2014.09.042

[18] Ata R. Artificial neural networks applications in wind energy systems: A review. Renewable and Sustainable Energy Reviews. 2015;49:534-562. DOI: 10.1016/j.rser.2015.04.166

[19] Jung J, Broadwater RP. Current status and future advances for wind speed and power forecasting. Renewable and Sustainable Energy Reviews. 2014;31:762-777. DOI: 10.1016/j.rser.2013.12.054

[20] Tascikaraoglu A, Uzunoglu M. A review of combined approaches for prediction of short-term wind speed and power. Renewable and Sustainable Energy Reviews. 2014;34:243-254. DOI: 10.1016/j.rser.2014.03.033

[21] Monfared M, Rastegar H, Kojabadi HM. A new strategy for wind speed forecasting using artificial intelligent methods. Renewable Energy. 2009;34(3):845-848. DOI: 10.1016/j. renene.2008.04.017

[22] Kavousi-Fard A, Khosravi A, Nahavandi S. A new fuzzy-based combined prediction interval for wind power forecasting. IEEE Transactions on Power Systems. 2016;31(1):18-26. DOI: 10.1109/TPWRS.2015.2393880
[23] Qiao W, Zhou W, Aller JM, Harley RG. Wind speed estimation based sensorless output maximization control for a wind turbine driving a DFIG. IEEE Transactions on Power Electronics. 2008;23(3):1156-1169. DOI: 10.1109/TPEL.2008.921185

[24] Chang TP, Liu FJ, Ko HH, Huang MC. Oscillation characteristic study of wind speed, global solar radiation and air temperature using wavelet analysis. Applied Energy. 2017;190:650-657. DOI: 10.1016/j. apenergy.2016.12.149

[25] Elhadidy MA. Performance evaluation of hybrid (wind/solar/ diesel) power systems. Renewable Energy. 2002;26(3):401-413. DOI: 10.1016/S0960-1481(01)00139-2

[26] Zhang L, Li Y. Optimal energy management of wind-battery hybrid power system with two-scale dynamic programming. IEEE Transactions on Sustainable Energy. 2013;4(3):765-773. DOI: 10.1109/TSTE.2013.2246875

[27] Coskun C, Oktay Z, Dincer I. Estimation of monthly solar radiation distribution for solar energy system analysis. Energy. 2011;36(2):1319-1323. DOI: 10.1016/j.energy.2010.11.009

[28] Notton G, Diaf S, Stoyanov L. Hybrid photovoltaic/wind energy systems for remote locations. Energy Procedia. 2011;6:666-677. DOI: 10.1016/j.egypro.2011.05.076

[29] Yang H, Wei Z, Chengzhi L. Optimal design and techno-economic analysis of a hybrid solar-wind power generation system. Applied Energy. 2009;86(2):163-169. DOI: 10.1016/j. apenergy.2008.03.008

[30] Sinha S, Chandel SS. Review of recent trends in optimization techniques for solar photovoltaic-wind based hybrid energy systems. Renewable and Sustainable Energy Reviews. 2015;50:755769. DOI: 10.1016/j.rser.2015.05.040 
[31] Abbassi A, Dami MA, Jemli M. A statistical approach for hybrid energy storage system sizing based on capacity distributions in an autonomous PV/ wind power generation system. Renewable Energy. 2017;103:81-93. DOI: 10.1016/j.renene.2016.11.024

[32] Arabali A, Ghofrani M, Etezadi-Amoli M, Fadali MS, Baghzouz Y. Genetic-algorithm-based optimization approach for energy management. IEEE Transactions on Power Delivery. 2013;28(1):162-170. DOI: 10.1109/TPWRD.2012.2219598

[33] Shadmand MB, Balog RS. Multiobjective optimization and design of photovoltaic-wind hybrid system for community smart DC microgrid. IEEE Transactions on Smart Grid. 2014;5(5):2635-2643. DOI: 10.1109/ tsg.2014.2315043

[34] Cho GJ, Oh YS, Kim MS, Kim JS, Kim CH, Mather B, et al. Optimal capacitor bank capacity and placement in distribution systems with high distributed solar power penetration. In: 2017 IEEE PES General Meeting; 16 July 2017; IEEE. 2017. pp. 1-5. DOI: 10.1109/ PESGM.2017.8273749

[35] Mahesh A, Sandhu KS. Hybrid wind/photovoltaic energy system developments: Critical review and findings. Renewable and Sustainable Energy Reviews. 2015;52:1135-1147. DOI: 10.1016/j.rser.2015.08.008

[36] Wu Z, Xia X. Optimal switching renewable energy system for demand side management. Solar Energy. 2015;114:278-288. DOI: 10.1016/j. solener.2015.02.001

[37] Sichilalu SM, Xia X. Optimal energy control of grid tied PV-dieselbattery hybrid system powering heat pump water heater. Solar Energy. 2015;115:243-254. DOI: 10.1016/j. solener.2015.02.028
[38] Li G, Chen Y, Li T. The realization of control subsystem in the energy management of wind/solar hybrid power system. In: 3rd Int. Conf. on Power Electronics Sys. and Applications (PESA); May 2009; Hong Kong, China. 2009. pp. $1-4$

[39] Olatomiwa L, Mekhilef S, Ismail MS. Energy management strategies in hybrid renewable energy systems: A review. Renewable and Sustainable Energy Reviews. 2016;62:821-835. DOI: 10.1016/j. rser.2016.05.040

[40] Sehar F, Pipattanasomporn M, Rahman S. Coordinated control of building loads, PVs and ice storage to absorb PEV penetrations. International Journal of Electrical Power \& Energy Systems. 2018;95:394-404. DOI: 10.1016/j.ijepes.2017.09.009

[41] Merabet A, Ahmed KT, Ibrahim H, Beguenane R, Ghias AM. Energy management and control system for laboratory scale microgrid based wind-PV-battery. IEEE Transactions on Sustainable Energy. 2017;8(1):145-154. DOI: 10.1109/ TSTE.2016.2587828

[42] Boukettaya G, Krichen L. A dynamic power management strategy of a grid connected hybrid generation system using wind, photovoltaic and flywheel energy storage system in residential applications. Energy. 2014;71:148-159. DOI: 10.1016/j.energy.2014.04.039

[43] Reihani E, Sepasi S, Roose LR, Matsuura M. Energy management at the distribution grid using a battery energy storage system (BESS). International Journal of Electrical Power \& Energy Systems. 2016;77:337-344. DOI: 10.1016/j.ijepes.2015.11.035

[44] Mohamed A, Mohammed O. Realtime energy management scheme for hybrid renewable energy systems in smart grid applications. Electric Power 
Systems Research. 2013;96:133-143. DOI: 10.1016/j.epsr.2012.10.015

[45] Lucas A, Chondrogiannis S. Smart grid energy storage controller for frequency regulation and peak shaving, using a vanadium redox flow battery. International Journal of Electrical Power \& Energy Systems. 2016;80:26-36. DOI: 10.1016/j.ijepes.2016.01.025

[46] Lawder MT, Suthar B, Northrop PW, De S, Hoff CM, Leitermann O, et al. Battery energy storage system (BESS) and battery management system (BMS) for grid-scale applications. Proceedings of the IEEE. 2014;102(6):1014-1030. DOI: $10.1109 /$ jproc.2014.2317451

[47] Nick M, Cherkaoui R, Paolone M. Optimal allocation of dispersed energy storage systems in active distribution networks for energy balance and grid support. IEEE Transactions on Power Systems. 2014;29(5):2300-2310. DOI: 10.1109/tpwrs.2014.2302020

[48] Gelazanskas L, Gamage KA. Demand side management in smart grid: A review and proposals for future direction. Sustainable Cities and Society. 2014;11:22-30. DOI: 10.1016/j. scs.2013.11.001

[49] Vasiljevska J, Lopes JP, Matos MA. Integrated micro-generation, load and energy storage control functionality under the multi micro-grid concept. Electric Power Systems Research. 2013;95:292-301. DOI: 10.1016/j. epsr.2012.09.014

[50] Yi Z, Dong W, Etemadi AH. A unified control and power management scheme for PV-battery-based hybrid microgrids for both grid-connected and islanded modes. IEEE Transactions on Smart Grid. 2017;9:1. DOI: 10.1109/ tsg.2017.2700332

[51] Abbassi R, Chebbi S. Energy management strategy for a gridconnected wind-solar hybrid system with battery storage: Policy for optimizing conventional energy generation. International Review of Electrical Engineering. 2012;7(2):3979-3990

[52] Singaravel MR, Daniel SA. MPPT with single DC-DC converter and inverter for grid-connected hybrid wind-driven PMSG-PV system. IEEE Transactions on Industrial Electronics. 2015;62(8):4849-4857. DOI: 10.1109 / tie.2015.2399277

[53] Alagoz BB, Kaygusuz A, Karabiber A. A user-mode distributed energy management architecture for smart grid applications. Energy. 2012;44(1):167-177. DOI: 10.1016/j. energy.2012.06.051

[54] Li X, Jiao X, Wang L. Coordinated power control of wind-PV-fuel cell for hybrid distributed generation systems. In: The SICE Annual Conference; September 2013; Nagoya, Japan: IEEE. 2013. pp. $150-155$

[55] Hong CM, Chen $\mathrm{CH}$. Intelligent control of a grid-connected windphotovoltaic hybrid power systems. International Journal of Electrical Power \& Energy Systems. 2014;55:554-561. DOI: 10.1016/j.ijepes.2013.10.024

[56] Chaurasia GS, Singh AK, Agrawal S, Sharma NK. A meta-heuristic firefly algorithm based smart control strategy and analysis of a grid connected hybrid photovoltaic/wind distributed generation system. Solar Energy. 2017;150:265-274. DOI: 10.1016/j. solener.2017.03.079

[57] Bendary AF, Ismail MM. Battery charge management for hybrid PV/ wind/fuel cell with storage battery. Energy Procedia. 2019;162:107-116. DOI: 10.1016/j.egypro.2019.04.012

[58] Qi W, Liu J, Christofides PD. Distributed supervisory predictive control of distributed wind and solar 
energy systems. IEEE Transactions on Control Systems Technology. 2013;21(2):504-512. DOI: $10.1109 /$ TCST.2011.2180907

[59] Baghaee HR, Mirsalim M, Gharehpetian GB, Talebi HA. A decentralized power management and sliding mode control strategy for hybrid AC/DC microgrids including renewable energy resources. IEEE Transactions on Industrial Informatics. 2017:1-1. DOI: 10.1109/tii.2017.2677943

[60] Gregory DC, Alesi LH, Crain JA. Distributed hybrid renewable energy power plant and methods, systems, and computer readable media for controlling a distributed hybrid renewable energy power plant [USA patent]; 2012

[61] Jun Z, Junfeng L, Jie W, Ngan HW. A multi-agent solution to energy management in hybrid renewable energy generation system. Renewable Energy. 2011;36(5):1352-1363. DOI: 10.1016/j.renene.2010.11.032

\section{[62] Xu Y, Zhang J, Wang W,} Juneja A, Bhattacharya S. Energy router: Architectures and functionalities toward energy internet. In: IEEE International Conference on Smart Grid Communications (SmartGridComm); 2011; Brussels, Belgium. 2011. pp. 31-36. DOI: $10.1109 /$ smartgridcomm.2011.610234

[63] Sechilariu M, Wang B, Locment F. Building integrated photovoltaic system with energy storage and smart grid communication. IEEE Transactions on Industrial Electronics. 2011;60(4):16071618. DOI: 10.1109/tie.2012.2222852

[64] Cao J, Yang M. Energy

internet-Towards smart grid 2.0. In: Fourth International Conference on Networking and Distributed Computing; 2013; Los Angeles, CA. 2013. pp. 105-110. DOI: $10.1109 /$ icndc. 2013.10
[65] Badwawi RA, Abusara M, Mallick T. A review of hybrid solar PV and wind energy system. Smart Science. 2015;3(3):127-138. DOI: 10.1080/23080477.2015.11665647

[66] Kabalci E. Design and analysis of a hybrid renewable energy plant with solar and wind power. Energy Conversion and Management. 2013;72:51-59. DOI: 10.1016/j. enconman.2012.08.027

[67] Available from: https://mnre.gov.in/ physical-progress-achievements

[68] Sawle Y, Gupta SC, Kumar Bohre A. PV-wind hybrid system: A review with case study. Cogent Engineering. 2016;3(1):1189305. DOI: 10.1080/23311916.2016.1189305

[69] Robinson P, Gowda AC, Sameer S, Patil S. Development of renewable energy based hybrid system for electricity generation-a case study. International Journal of Latest Technology in Engineering, Management \& Applied Science. 2017;VI(VIIIS):46-52

[70] Fathima H, Palanisamy K. Optimized sizing, selection, and economic analysis of battery energy storage for grid-connected wind-PV hybrid system. Modelling and Simulation in Engineering. 2015;2015:16. DOI: $10.1155 / 2015 / 713530$

[71] Hittinger E, Whitacre JF, Apt J. What properties of grid energy storage are most valuable? Journal of Power Sources. 2012;206:436-449. DOI: 10.1016/j.jpowsour.2011.12.003

[72] Srivastava AK, Kumar AA, Schulz NN. Impact of distributed generations with energy storage devices on the electric grid. IEEE Systems Journal. 2012;6(1):110-117. DOI: 10.1109/jsyst.2011.2163013

[73] Korada N, Mishra MK. Grid adaptive power management strategy 
for an integrated microgrid with hybrid energy storage. IEEE Transactions on Industrial Electronics. 2017;64(4):28842892. DOI: $10.1109 /$ tie.2016.2631443

[74] Shu Z, Jirutitijaroen P. Optimal operation strategy of energy storage system for grid-connected wind power plants. IEEE Transactions on Sustainable Energy. 2016;5(1):190-199. DOI: $10.1109 /$ tste.2013.2278406

[75] Salas DF, Powell WB. Benchmarking a scalable approximate dynamic programming algorithm for stochastic control of grid-level energy storage. INFORMS Journal on Computing. 2018;30(1):106-123. DOI: 10.1287/ ijoc. 2017.0768

[76] Madlener R, Latz J. Economics of centralized and decentralized compressed air energy storage for enhanced grid integration of wind power. Applied Energy. 2013;101:299-309. DOI: 10.1016/j. apenergy.2011.09.033

[77] Koller M, Borsche T, Ulbig A, Andersson G. Review of grid applications with the Zurich 1MW battery energy storage system. Electric Power Systems Research. 2015;120:128135. DOI: 10.1016/j.epsr.2014.06.023

[78] Kroposki B, Johnson B, Zhang Y, Gevorgian V, Denholm P, Hodge BM, et al. Achieving a $100 \%$ renewable grid: Operating electric power systems with extremely high levels of variable renewable energy. IEEE Power and Energy Magazine. 2017;15(2):61-73. DOI: $10.1109 / \mathrm{mpe} .2016 .2637122$

[79] Malysz P, Sirouspour S, Emadi A. An optimal energy storage control strategy for grid-connected microgrids. IEEE Transactions on Smart Grid. 2014;5(4):1785-1796. DOI: 10.1109/tsg.2014.2302396

[80] Müller M, Viernstein L, Truong CN, Eiting A, Hesse HC, Witzmann R, et al.
Evaluation of grid-level adaptability for stationary battery energy storage system applications in Europe. Journal of Energy Storage. 2017;9:1-11. DOI: 10.1016/j.est.2016.11.005

[81] Bose S, Gayme DF, Topcu U, Chandy KM. Optimal placement of energy storage in the grid. In: 51st IEEE Conference on Decision and Control (CDC); IEEE 2012; Maui, HI, USA. pp. 5605-5612. DOI: 10.1109/ cdc. 2012.6426113

[82] Bhandari B, Lee KT, Lee CS, Song CK, Maskey RK, Ahn SH. A novel off-grid hybrid power system comprised of solar photovoltaic, wind, and hydro energy sources. Applied Energy. 2014;133:236-242. DOI: 10.1016/j. apenergy.2014.07.033

[83] Hearn CS, Lewis MC, Pratap SB, Hebner RE, Uriarte FM, Chen D, et al. Utilization of optimal control law to size grid-level flywheel energy storage. IEEE Transactions on Sustainable Energy. 2013;4(3):611-618. DOI: 10.1109/ tste.2013.2238564

[84] Denholm P, Hand M. Grid flexibility and storage required to achieve very high penetration of variable renewable electricity. Energy Policy. 2011;39(3):1817-1830. DOI: 10.1016/j. enpol.2011.01.019

[85] Kerestes RJ, Reed GF, Sparacino AR. Economic analysis of grid level energy storage for the application of load leveling. In: 2012 IEEE PES Gen Meeting; 2012; San Diego, CA, USA. 2012. pp. 1-9. DOI: 10.1109/ pesgm.2012.6345072

[86] Williams CJ, Binder JO, Kelm T. Demand side management through heat pumps, thermal storage and battery storage to increase local selfconsumption and grid compatibility of PV systems. In: 3rd IEEE PES Innovative Smart Grid Technologies Europe (ISGT Europe); 2012; Berlin, 
Germany. 2012. pp. 1-6. DOI: 10.1109/ isgteurope.2012.6465874

[87] Zhao H, Wu Q, Hu S, Xu H. Review of energy storage system for wind power integration support. Applied Energy. 2015;137:545-553. DOI: $10.1016 /$ j. apenergy.2014.04.103

[88] Ferreira HL, Garde R, Fulli G, Kling W, Lopes JP. Characterization of electrical energy storage technologies. Energy. 2013;53:288-298. DOI: 10.1016/j. energy.2013.02.037

[89] Luo X, Wang J, Dooner M, Clarke J. Overview of current development in electrical energy storage technologies and the application potential in power system operation. Applied Energy. 2015;137:511-536. DOI: $10.1016 / j$. apenergy.2014.09.081

[90] Dunn B, Kamath H, Tarascon JM. Electrical energy storage for the grid: A battery of choices. Science. 2011;334(6058):928-935. DOI: 10.1126/ science.1212741

[91] Palizban O, Kauhaniemi K. Energy storage systems in modern gridsMatrix of technologies and applications. Journal of Energy Storage. 2016;6:248259. DOI: 10.1016/j.est.2016.02.001

[92] Knap V, Sinha R, Swierczynski M, Stroe DI, Chaudhary S. Grid inertial response with Lithium-ion battery energy storage systems. In: IEEE 23rd International Symposium on Industrial Electronics (ISIE); 2014; Istanbul, Turkey. 2014. pp. 1817-1822. DOI: 10.1109/isie.2014.6864891

[93] Leadbetter J, Swan LG. Selection of battery technology to support gridintegrated renewable electricity. Journal of Power Sources. 2012;216:376-386. DOI: 10.1016/j.jpowsour.2012.05.081

[94] Wang K, Jiang K, Chung B, Ouchi T, Burke PJ, Boysen DA, et al. Lithiumantimony-lead liquid metal battery for grid-level energy storage. Nature. 2014;514(7522):348-350. DOI: 10.1038/ nature 13700

[95] Sbordone D, Bertini I, Di

Pietra B, Falvo MC, Genovese A, Martirano L. EV fast charging stations and energy storage technologies: A real implementation in the smart micro grid paradigm. Electric Power Systems Research. 2014;120:96-108. DOI: 10.1016/j.epsr.2014.07.033

[96] Richardson DB. Electric vehicles and the electric grid: A review of modeling approaches, impacts, and renewable energy integration. Renewable and Sustainable Energy Reviews. 2013;19:247-254. DOI: 10.1016/j.rser.2012.11.042

[97] Schroeder A. Modeling storage and demand management in power distribution grids. Applied Energy. 2011;88(12):4700-4712. DOI: 10.1016/j. apenergy.2011.06.008

[98] Clement-Nyns K, Haesen E, Driesen J. The impact of vehicleto-grid on the distribution grid. Electric Power Systems Research. 2011;81(1):185-192. DOI: 10.1016/j. epsr.2010.08.007

[99] Pang C, Dutta P, Kezunovic M. BEVs/PHEVs as dispersed energy storage for V2B uses in the smart grid. IEEE Transactions on Smart Grid. 2012;3(1):473-482. DOI: 10.1109/ tsg.2011.2172228

[100] Sun J, Li M, Zhang Z, Xu T, $\mathrm{He}$ J, Wang H, et al. Renewable energy transmission by HVDC across the continent: System challenges and opportunities. CSEE Journal of Power and Energy Systems. 2017;3(4):353-364. DOI: 10.17775/ CSEEJPES.2017.01200

[101] Trevisani L, Fabbri M, Negrini F. Long distance renewable energy sources power transmission 
using hydrogen-cooled MgB2

superconducting line. Cryogenics.

2007;47(2):113-120. DOI: 10.1016/j.

cryogenics.2006.10.002

[102] Oyedepo SO, Agbetuyi AF,

Odunfa KM. Transmission network enhancement with renewable energy.

Journal of Fundamentals of Renewable

Energy and Applications. 2014;5(5):1-1.

DOI: $10.4172 / 20904541.1000145$ 


\title{
Solar Thermochemical Fuel Generation
}

\author{
Hongsheng Wang
}

\begin{abstract}
Solar energy is one of the most abundant, clean, and widespread energy in the world, which has the potential to address the issues of environmental pollution, global warming, and energy crisis, while the intermittent distribution of solar energy in time and space limits its utilization. Among various approaches of solar energy utilization, converting solar energy into chemical fuel (e.g., hydrogen) by thermochemical approach could maintain the steady and high-efficient energy supply and can make use of the full-spectrum solar energy. The research about solar thermochemical fuel generation lasts more than 40 years, and lots of reaction system and reactors have been proposed. This chapter reviews the state-of-the-art progress of solar thermochemical fuel generation, and the characteristics of different systems have been compared and discussed, which may give systematical insight into the development and improvement of solar fuel generation by thermochemical approach in the future.
\end{abstract}

Keywords: solar energy, solar fuel, solar thermochemistry, thermochemical fuel, solar membrane reactor

\section{Introduction}

\subsection{Energy and environmental problems and solutions}

Due to population growth and rapid industrial development, world energy demand has increased significantly. Compared with the previous generation, the world's population has increased rapidly by 2 billion [1], and this mainly comes from the population growth of developing countries, and rapid population growth poses more severe challenges to increasingly scarce energy and resource supplies. The importance of energy for social development is self-evident. In order to ensure the supply of energy, a large amount of fossil energy is used, which at the same time has a serious impact on the environment, leading to increasingly serious problems of atmospheric pollution and the greenhouse effect. Therefore, improving energy efficiency and using more clean energy and exploring a sustainable development path compatible with energy use and the environment have become one of the important topics in energy science research.

The efficient use of renewable energy is of great significance. Among the many renewable energy sources, solar energy has become one of the best choices for future energy sources with its unique advantages, which is the most abundant renewable energy source and widely distributed. 


\subsection{Advantages of solar thermochemical fuel generation}

Solar energy is a huge amount of clean energy. It is of great significance to develop and utilize solar energy reasonably and efficiently. However, the efficient use of solar energy also faces limitations, such as the low energy density of solar energy, the unstable energy supply, the discontinuous time and spatial distribution of solar radiation, and the difficulty of direct storage [2, 3]. Therefore, solar energy is converted into chemical energy stored in fuels, which is generally considered to be an effective solution to make up for solar defects [2-6].

There are mainly four approaches for converting solar energy into chemical energy to generate solar fuel, which is illustrated in Figure 1. The photobiological process is limited by the low energy conversion efficiency now, and it is still at a very early stage of the development [7]. The other three methods have their own properties and have attracted lots of attention. Photo-electrolysis approach is most convenient, but it is also limited by the conversion rate, and researchers are seeking for the catalysts which have better performance. The electrolysis using photovoltaic (PV) materials and electrolyzer is the most mature approach for producing solar fuel. However, the PV materials can only utilize the light with a certain range of wavelength (usually short wavelength light), and the other part of sunlight absorbed is converted into thermal energy, which is wasted as residual heat, leading to a limited PV cell efficiency (the commercial PV cell efficiency is about $15 \%$; the highest multiple-junction PV cell efficiency in lab is higher than $40 \%$ with high cost). The total energy efficiency from solar energy to chemical energy is the product of solar power efficiency (e.g., PV cell efficiency) and electrolysis efficiency, so the total efficiency has potential to be further improved. Compared with electrolysis, solar fuel generation by thermochemistry can utilize the sunlight with whole solar spectrum, which has a high theoretical energy efficiency. So the solar thermochemical fuel generation is a promising method and will be discussed in this chapter in details.

Figure 2 is a schematic diagram of the solar thermochemical energy conversion process. Solar energy with lower energy density is received by solar collectors and converted into solar thermal energy. Solar thermal energy enters the absorber through heat transfer and drives the chemical reaction, so that low-energy-density solar energy is stored in the form of solar fuel as chemical energy with high energy density, which is relatively easy for storage and transportation. The sustainable and stable use of solar energy is achieved by transporting solar fuel to remote and needed places for power generation and chemical processes, etc., and solving the

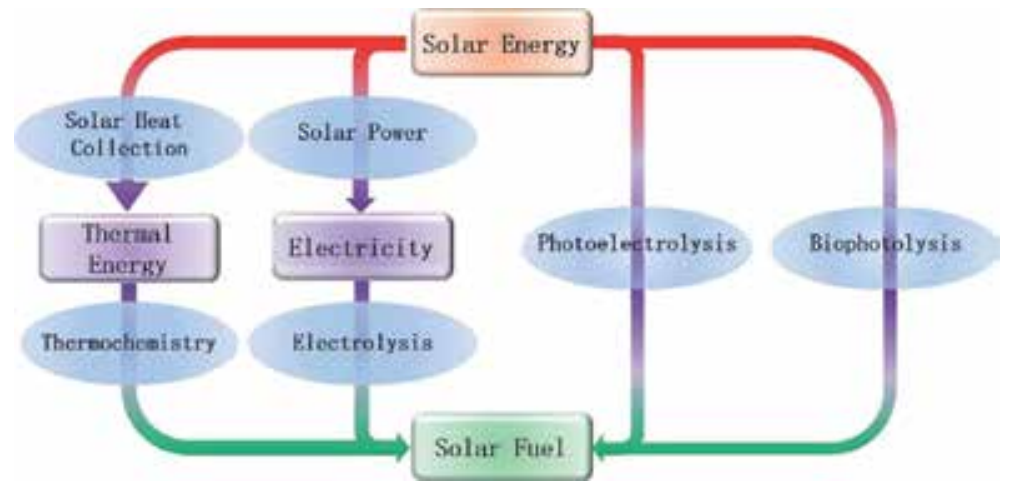

Figure 1.

Illustration of solar fuel via various approaches. 


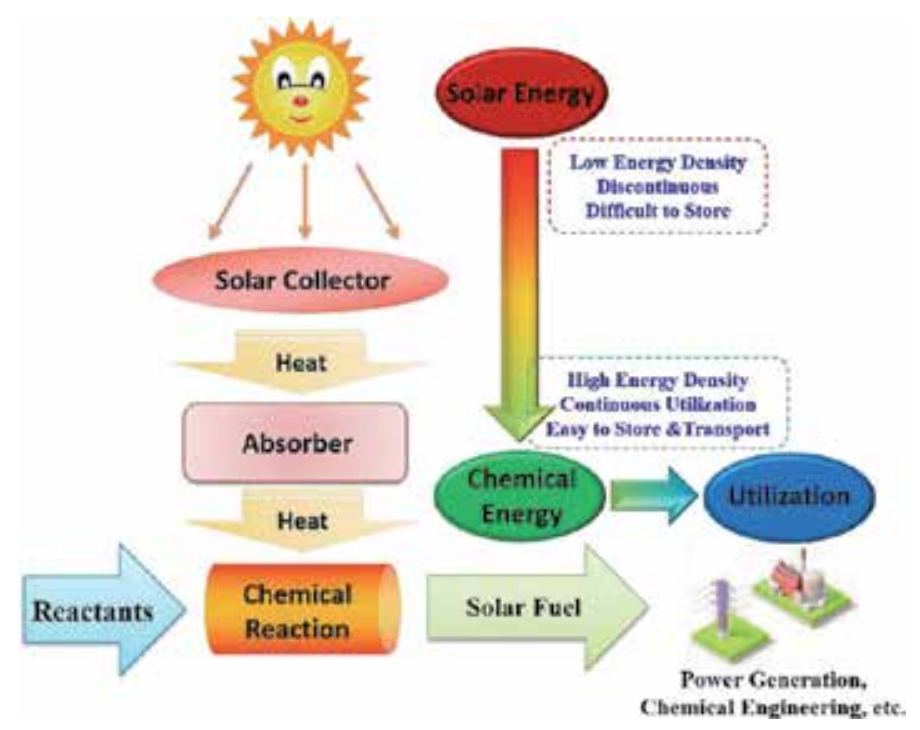

Figure 2.

Illustration of solar thermochemical energy conversion process.

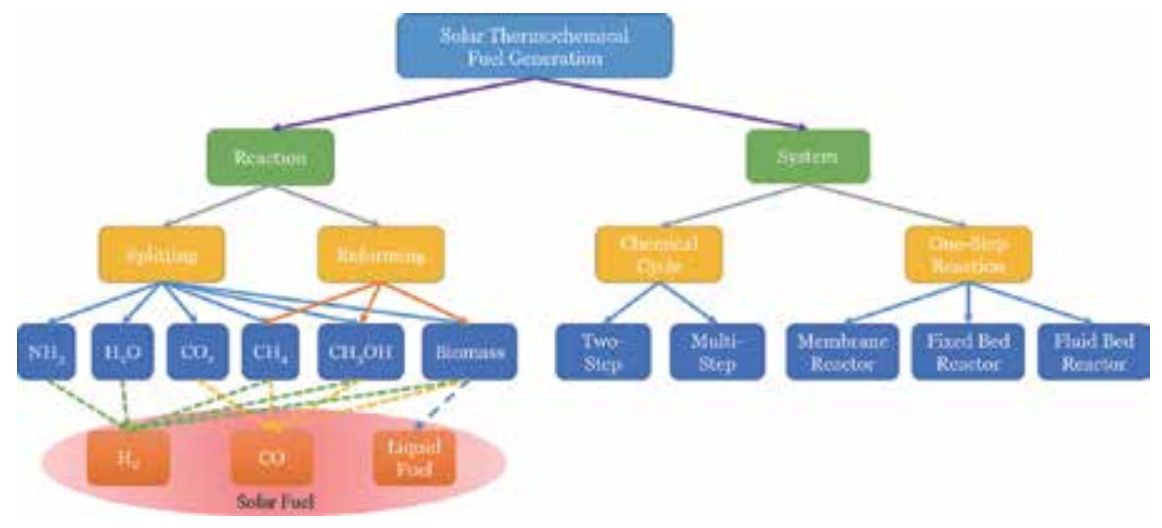

Figure 3.

Classification of solar thermochemical fuel generation.

discontinuity of solar distributed in time and space by means of chemical energy storage.

There are many researches about the reaction and system for solar thermochemical fuel generation published, and some of the significant parts have been classified in Figure 3. The two main fuels from solar energy are hydrogen and carbon monoxide, which both have great higher heating values and are potential to be utilized in the future, especially hydrogen, as hydrogen has the following characteristics:

1. Rich hydrogen energy reserves. On the earth, hydrogen mainly exists in the form of hydrocarbons and water, and more than $70 \%$ of the earth's surface is covered by water. Therefore, the earth contains a huge amount of hydrogen and has great potential for development.

2. The energy density of hydrogen is large. The higher heating value of hydrogen is much higher than that of hydrocarbons and alcohol compounds, and the consumption of hydrogen energy is increasing every year. 
3. Hydrogen is renewable. Hydrogen can be obtained from water, and the oxidation of hydrogen produces water. Therefore, the hydrogen combustion and energy release cycle does not consume other substances.

4. Hydrogen energy is clean energy. Whether hydrogen is consumed by direct combustion or fuel cell power generation, the only product is water, without any waste pollution, which is clean and environmentally friendly.

5. Hydrogen is relatively easy to be converted and stored. Compared with other energy sources such as solar energy, wind energy, electrical energy, and thermal energy, hydrogen is a chemical raw material and is easily to be converted into hydrocarbons for storage, thereby expanding the scope of hydrogen energy in time and space.

\subsection{Thermodynamic of solar thermochemical process}

Different chemical reaction processes require different temperatures, so it is necessary to match the solar thermal energy temperature with the chemical reaction temperature for efficient energy utilization. Different solar thermal temperatures need to be achieved with different forms of solar collectors for matching various chemical reactions.

The heat collection temperature of solar collectors depends on many factors, but the most important factor is the concentration ratio, which is the ratio of the total area of the opening of the collector mirror field to the spot area on the focal plane. Concentration ratio is an important parameter for designing concentrating solar thermal utilization. Under the same conditions, the higher the concentration ratio, the higher the heat collection temperature. In a unit of time, the energy emitted by a black body per unit area is proportional to the fourth power of its temperature, and solar energy is close to a $6000 \mathrm{~K}$ black body, so the radiant energy it emits is:

$$
Q_{\mathrm{s}}=4 \pi r^{2} \sigma T_{\mathrm{s}}^{4}
$$

Among them, $T_{\mathrm{s}}$ is the absolute surface temperature of the sun and $\sigma$ is the StefanBoltzmann constant. If the orbit of the earth is regarded as a circle with a radius $R$, as shown in Figure 4, the energy that $Q_{s}$ throws on the absorber of area $A$ is:

$$
Q_{\mathrm{S} \rightarrow \mathrm{A}}=A \cdot \frac{Q_{\mathrm{s}}}{4 \pi R^{2}}
$$

After the absorber absorbs energy, the temperature will rise. Assuming the temperature rises to $T_{\mathrm{a}}$, if the conduction and convection losses are ignored, the absorber will radiate energy, given as:

$$
Q_{\mathrm{a}}=a \sigma T_{\mathrm{a}}^{4}
$$

According to the second law of thermodynamics, heat can only be transferred spontaneously from a high-temperature object to a low-temperature object, so the temperature $T_{\mathrm{a}}$ of the absorber is always less than or equal to the solar surface temperature. In the limit, the two temperatures are equal, that is, $T_{\mathrm{a}}=T_{\mathrm{s}}$, and the amount of heat absorbed by the device is equal to the amount of radiation:

$$
Q_{\mathrm{S} \rightarrow \mathrm{A}}-Q_{\mathrm{a}}=A \cdot \frac{Q_{s}}{4 \pi R^{2}}-a \sigma T_{a}^{4}=0
$$




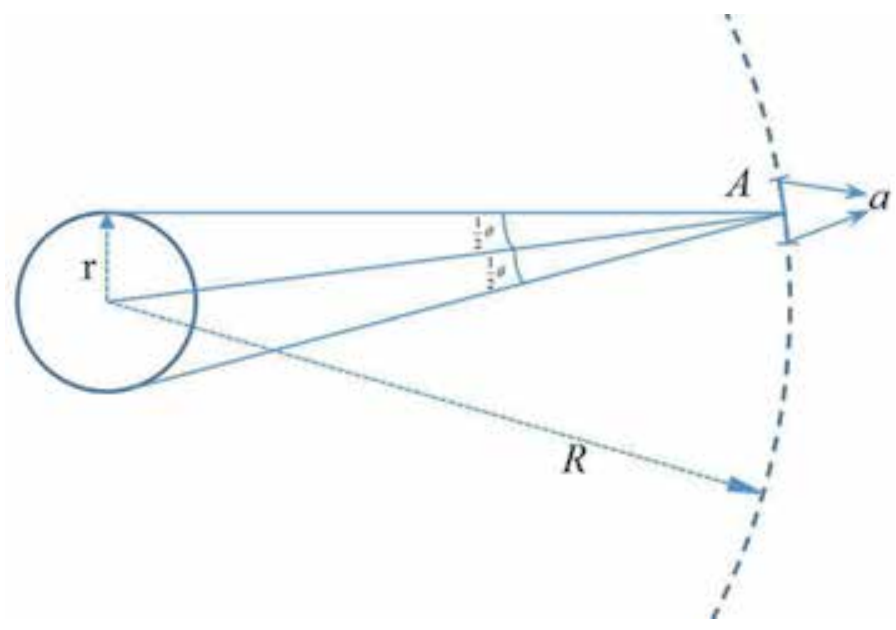

Figure 4.

Illustration of solar radiation trajectory.

According to the definition of the concentration ratio, it is:

$$
C=\frac{A}{a}=\frac{R^{2}}{r^{2}}=\frac{1}{\sin ^{2} \frac{\theta}{2}}
$$

Among them, $\theta$ is the opening angle of the sun, and the value is $32^{\prime}$, so the theoretical limiting concentration ratio is 45,000 . In practical applications, the light ratio is much lower than the theoretical condenser ratio, due to manufacturing errors (misfocus, specular errors, etc.), structural disturbances, unsatisfactory optical properties (specular reflectance, glass absorptivity, etc.), shadows and sun tracking, etc.

In the actual application process, there are two types of common solar concentrating forms: linear focusing and point focusing. Among them, linear focusing solar collectors include parabolic trough solar collectors and linear Fresnel solar collectors. Because the collectors have different heat collection methods, they also have different light collection ratios and heat collection temperatures. Because pointfocused solar collector focuses in two dimensions and line-focused solar collector focuses in one-dimensional directions, point-focused solar collectors usually have a larger concentration ratio, which could approach a greater temperature. However, high temperature usually means higher requirements for materials and processing industries, higher radiation losses, and heat costs of the collector. Table 1 lists the typical solar thermal power generation mirror field parameters. In thermal power plants, a higher temperature for power generation will allow the Rankine cycle to have a higher Carnot efficiency, leading to a greater power generation efficiency.

In the process of collecting solar energy by using a solar collector, energy is dissipated due to radiation. The absorption efficiency is defined as the ratio of the solar energy absorbed by the absorption cavity to the total solar energy projected by the collector into the absorption cavity, given as [18]:

$$
\eta_{a b s}=\frac{I A \eta_{\mathrm{A}} \alpha-a \varepsilon \sigma T^{4}}{I A}
$$

$\eta_{a b s}$ is the absorption efficiency; $I$ is the solar radiation intensity; $A$ is the area of the condenser; $\eta_{\mathrm{A}}$ is the optical efficiency; $\alpha$ and $\varepsilon$ are the absorptance and emissivity of the absorption cavity; $a$ is the area of the absorber; $\sigma$ is the Stefan-Boltzmann 


\begin{tabular}{lcccc}
\hline Type & $\begin{array}{c}\text { Annual power } \\
\text { generation } \\
\text { efficiency }(\%)\end{array}$ & $\begin{array}{c}\text { Peak } \\
\text { efficiency } \\
(\%)\end{array}$ & $\begin{array}{c}\text { Operating } \\
\text { temperature } \\
\left({ }^{\circ} \mathbf{C}\right)\end{array}$ & $\begin{array}{c}\text { Concentration } \\
\text { ratio }\end{array}$ \\
\hline $\begin{array}{l}\text { Parabolic trough } \\
\text { collector power plant }\end{array}$ & 14 & 25 & 400 & $30-100$ \\
\hline $\begin{array}{l}\text { Linear Fresnel } \\
\text { collector power plant }\end{array}$ & 13 & 18 & $300-400$ & 30 \\
\hline $\begin{array}{l}\text { Disc collector power } \\
\text { plant }\end{array}$ & 20 & 32 & $550-750$ & $1000-10,000$ \\
\hline $\begin{array}{l}\text { Tower collector power } \\
\text { plant }\end{array}$ & 16 & 22 & $400-600$ & $500-5000$ \\
\hline
\end{tabular}

Table 1.

Performance parameters of typical solar collector fields [8-17].

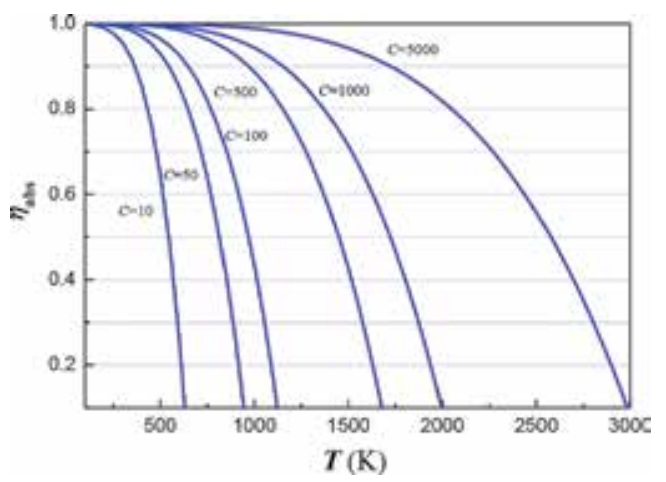

Figure 5.

The relationship among the ideal absorption efficiency of the collector, the concentration ratio, and the heat collection temperature.

constant $\left(5.67 \times 10^{-8} \mathrm{~W} /\left(\mathrm{m}^{2} \times \mathrm{K}^{4}\right)\right)$; and $T$ is the set thermal temperature. If it is assumed that the absorption cavity is black body, then $\eta_{\mathrm{A}}, \alpha$, and $\varepsilon$ are all 1 , the above formula can be simplified as:

$$
\eta_{a b s}=1-\frac{\sigma T^{4}}{I C}
$$

where $C$ is the concentration ratio. If $I=1000 \mathrm{~W} / \mathrm{m}^{2}$, the relationship curve between the ideal absorption efficiency of the collector, the concentration ratio, and heat collection temperature can be obtained through calculation, as shown in Figure 5. When the heat collection temperature is fixed, the heat collection efficiency increases with the increase of the concentration ratio; when the concentration ratio is determined, the heat collection efficiency is a decreasing function of the heat collection temperature, mainly because as the heat collection temperature increases, the temperature difference between reactor and environment rises up, leading to an increase in radiation loss, which reduces the efficiency of heat collection.

With multiplying the obtained absorption efficiency by the Carnot cycle efficiency, the system efficiency can be obtained, which is the maximum theoretical conversion efficiency from the solar thermal energy obtained to work or electricity [18]:

$$
\eta_{\mathrm{s}}=\left(\frac{T_{\mathrm{H}}-T_{0}}{T_{\mathrm{H}}}\right)\left(1-\frac{\sigma T_{\mathrm{H}}{ }^{4}}{I C}\right)
$$




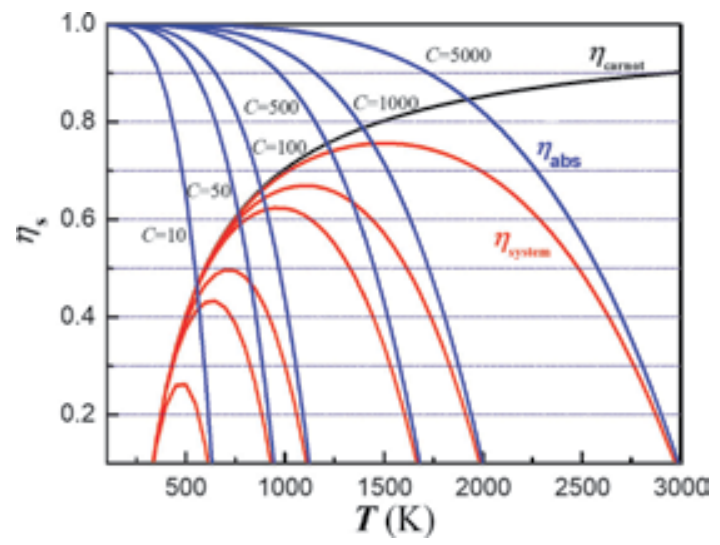

Figure 6.

The relationship among the maximum efficiency from solar thermal energy to work, the concentration ratio, and the heat collection temperature.

where $T_{\mathrm{H}}$ is the heat collection temperature of the solar collector and $T_{0}$ is the ambient temperature. The calculation results are shown in Figure 6.

When decomposing water to produce hydrogen without relying on fossil energy, the temperature required for thermochemical reactions is about $1300-1800^{\circ} \mathrm{C}$. According to Figures 5 and 6 , it can be seen that a tower or dish collector with a concentration ratio of 5000 should be selected. When using fossil fuel (e.g., methane) to split water for hydrogen generation, the reaction temperature could be decreased to $700-1000^{\circ} \mathrm{C}$. A tower or dish solar concentrator with a concentration ratio of 1000 should be used. The reaction temperature of the novel solar hydrogen permeation membrane alternating cycle methane reforming system introduced later in this chapter is about $350-400^{\circ} \mathrm{C}$, and a trough solar concentrator with a concentration ratio of $80-100$ is enough for it, which has a much lower cost compared with tower or the dish-type solar concentrating collector.

According to Eq. (8), when the concentration ratio $C$ is given, the first-order derivative function of can be obtained, shown as Eq. (9):

$$
\frac{\mathrm{d} \eta_{s}}{\mathrm{~d} T}=\frac{T_{0}}{T_{\mathrm{H}}{ }^{2}}+\frac{\sigma T_{\mathrm{H}}^{2}\left(3 T_{0}-4 T_{\mathrm{H}}\right)}{I C}
$$

By maintaining Eq. (9) equal to 0, the optimal heat collection temperature can be obtained at a given concentration ratio, and the optimal heat collection

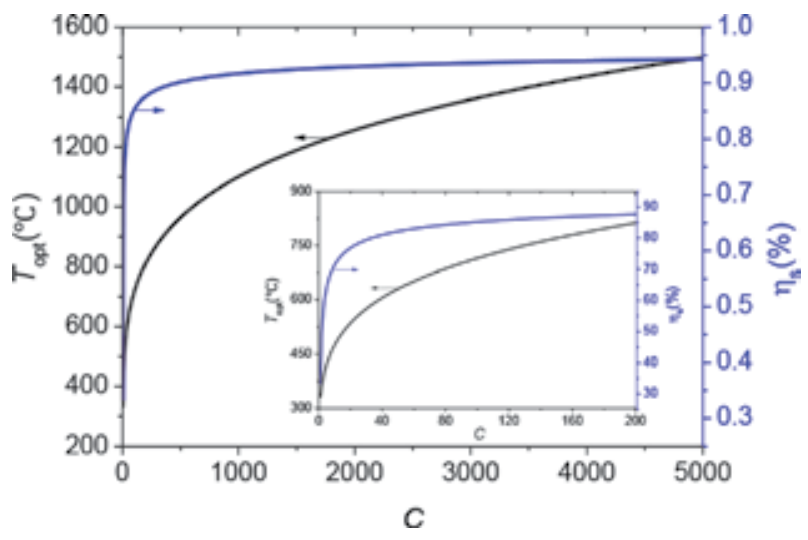

Figure 7.

Variation of maximum theoretical efficiency from solar energy to work and optimal thermal energy collection temperature with concentration ratio. 
temperature can be substituted into Eq. (8) to obtain the sun at the best heat collection temperature. The maximum theoretical efficiency from solar energy to work is shown in Figure 7.

From Figure 7, as the concentration ratio increases, the intensity of radiation received per unit area of the collector increases, so both the optimal heat collection temperature and the maximum theoretical efficiency increase. Because the solar collector has a fixed concentration ratio in practical applications, Figure 7 has guiding significance for determining the optimal heat-collecting temperature for a solar heat collector with a specific concentration ratio. The solar thermal energy of the system has the maximum work efficiency at the best concentration ratio.

\section{Thermochemical cycle}

The existing thermochemical cycle for hydrogen production mainly includes metal oxide system thermochemical cycle, sulfur-containing system, sulfuric acid decomposition method, metal-halide system, and reformed methane hydrogen production. All of the thermochemical cycles could be classified as multi-step thermochemical cycles and two-step thermochemical cycles.

\subsection{Multi-step thermochemical cycles}

\subsubsection{Hydrogen generation system containing sulfur}

There are four main types of hydrogen production in sulfur-containing systems: iodine-sulfur cycle, $\mathrm{H}_{2} \mathrm{SO}_{4}-\mathrm{H}_{2} \mathrm{~S}$ cycle, sulfuric acid-methanol cycle, and sulfate cycle. Among them, the iodine-sulfur cycle is the most famous. It was invented by the United States GA company in the 1970s, so it is also called the GA cycle. The process is shown in Figure 8. The main reaction process is as follows:

$$
\begin{gathered}
\mathrm{SO}_{2}+\mathrm{I}_{2}+2 \mathrm{H}_{2} \mathrm{O} \rightarrow 2 \mathrm{HI}+\mathrm{H}_{2} \mathrm{SO}_{4} \\
\mathrm{H}_{2} \mathrm{SO}_{4} \rightarrow \mathrm{H}_{2} \mathrm{O}+\mathrm{SO}_{2}+\frac{1}{2} \mathrm{O}_{2} \\
2 \mathrm{HI} \rightarrow \mathrm{H}_{2}+\mathrm{I}_{2} \\
\mathrm{H}_{2} \mathrm{O} \rightarrow \mathrm{H}_{2}+\frac{1}{2} \mathrm{O}_{2}
\end{gathered}
$$

GA company found [19] that the excess $\mathrm{I}_{2}$ exists, and $\mathrm{HI}$ and $\mathrm{H}_{2} \mathrm{SO}_{4}$ can be separated into two liquid phases, which is the basis for the development of the IS cycle. The advantages of the IS cycle are using of thermal energy below $1000^{\circ} \mathrm{C}$ for hydrogen generation, closed circuit, only water being needed to be added in the circulation process, and the expected efficiency which can reach 52\%. The disadvantages are concentrated sulfuric acid being highly corrosive when heated at high temperature; the equilibrium decomposition ratio of $\mathrm{HI}$ being low $(20 \%)$; and the reaction intermediate products sulfur dioxide and iodine being easy to cause pollution and liable to have side reactions.

\subsubsection{Sulfuric acid decomposition method}

This type of method is best known as the Westinghouse cycle [20], and its main process is shown in Figure 9. The highest temperature in the process needs to be above $800^{\circ} \mathrm{C}$, and the efficiency of the cycle can reach $40 \%$. If multi-stage electrolysis is used, it can reach $46 \%$. However, the disadvantage is that concentrated 
sulfuric acid is highly corrosive at high temperatures and has high requirements for material selection.

\subsubsection{Metal-halide system}

The most famous in this system is the UT-3 cycle proposed by the University of Tokyo. The main process is as follows:

$$
\begin{gathered}
\mathrm{CaBr}_{2}+\mathrm{H}_{2} \mathrm{O} \stackrel{1033 \mathrm{~K}}{\longrightarrow} \mathrm{CaO}+2 \mathrm{HBr} \\
\mathrm{CaO}+\mathrm{Br}_{2} \stackrel{845 \mathrm{~K}}{\longrightarrow} \mathrm{CaBr}_{2}+\frac{1}{2} \mathrm{O}_{2} \\
\mathrm{Fe}_{3} \mathrm{O}_{4}+8 \mathrm{HBr} \stackrel{493 \mathrm{~K}}{\longrightarrow} 3 \mathrm{FeBr}_{2}+4 \mathrm{H}_{2} \mathrm{O}+\mathrm{Br}_{2} \\
3 \mathrm{FeBr}_{2}+4 \mathrm{H}_{2} \mathrm{O} \stackrel{833 \mathrm{~K}}{\longrightarrow} \mathrm{Fe}_{3} \mathrm{O}_{4}+6 \mathrm{HBr}+\mathrm{H}_{2}
\end{gathered}
$$

Sakurai [21] found that the hydrolysis of calcium bromide was the slowest during this cycle, because the calcium oxide agglomerated, reducing the reaction interface area. The addition of lauric acid as a foaming agent for dispersing the calcium oxide aggregates can improve the performance of the reaction. The Argonne National Laboratory in the United States has also researched and developed this process [22]. Its main feature is the decomposition or formation of

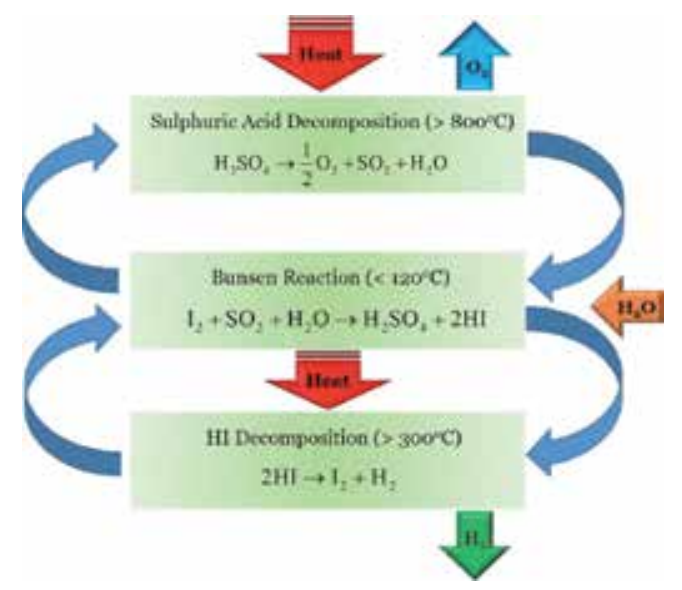

Figure 8.

Illustration of iodine-sulfur cycle.

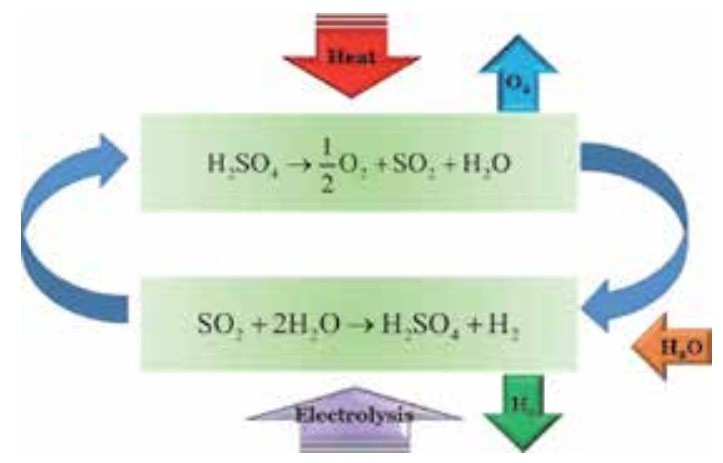

Figure 9.

Westinghouse cycle diagram. 


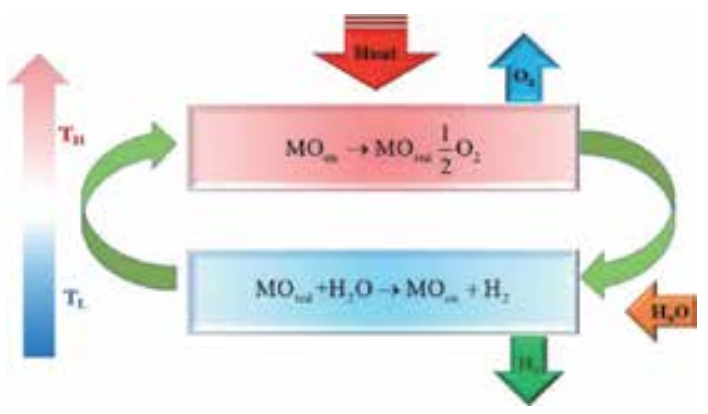

Figure 10.

Metal oxide thermochemical cycle for hydrogen production.

HBr by electrolytic method or "cold" plasma method. This reaction has the following advantages: its expected thermal efficiency is $35-40 \%$, and if the power is generated at the same time, the overall efficiency can be improved by $10 \%$; the two-step key reaction is a gas-solid reaction, which significantly simplifies the separation of products and reactants; the elements used are cheap and readily available; the process involves only solid and gaseous reactants and products. However, the separation of intermediate products in the reaction process is also a problem and challenge in the process.

\subsection{Two-step thermochemical cycles}

The common two-step thermochemical cycle hydrogen production process is mainly metal oxide thermochemical cycle, which has the following three forms:

Oxide:

$$
\begin{gathered}
\mathrm{XO} \rightarrow \mathrm{X}+0.5 \mathrm{O}_{2} \\
\mathrm{X}+\mathrm{H}_{2} \mathrm{O} \rightarrow \mathrm{XO}+\mathrm{H}_{2} \\
\text { or } \\
\frac{1}{\delta} \mathrm{XO}_{2} \rightarrow \frac{1}{\delta} \mathrm{XO}_{2-\delta}+\frac{1}{2} \mathrm{O}_{2} \\
\frac{1}{\delta} \mathrm{XO}_{2-\delta}+\mathrm{H}_{2} \mathrm{O} \rightarrow \frac{1}{\delta} \mathrm{XO}_{2}+\mathrm{H}_{2}
\end{gathered}
$$

Hydride:

$$
\begin{gathered}
\mathrm{XH}_{2} \rightarrow \mathrm{X}+\mathrm{H}_{2} \\
\mathrm{X}+\mathrm{H}_{2} \mathrm{O} \rightarrow \mathrm{XH}_{2}+0.5 \mathrm{O}_{2}
\end{gathered}
$$

Hydroxide:

$$
\begin{gathered}
2 \mathrm{XOH} \rightarrow 2 \mathrm{X}+\mathrm{H}_{2} \mathrm{O}+0.5 \mathrm{O}_{2} \\
2 \mathrm{X}+2 \mathrm{H}_{2} \mathrm{O} \rightarrow 2 \mathrm{XOH}+\mathrm{H}_{2}
\end{gathered}
$$

Among them, metal oxide thermochemical hydrogen production is the most common. The process is shown in Figure 10.

As shown in Figure 10, metal oxides are reduced by releasing oxygen at high temperatures, and oxidized with water at low temperatures, taking away oxygen 
atoms from water molecules to generate hydrogen. During the thermochemical cycle, metal oxides can be reduced to simple metals, such as:

$$
\begin{gathered}
\mathrm{ZnO} \rightarrow \mathrm{Zn}+\frac{1}{2} \mathrm{O}_{2} \\
\mathrm{Zn}+\mathrm{H}_{2} \mathrm{O} \rightarrow \mathrm{ZnO}+\mathrm{H}_{2}
\end{gathered}
$$

Metal oxides may also be reduced from higher valence to lower valence oxides, such as:

$$
\begin{gathered}
\mathrm{Fe}_{3} \mathrm{O}_{4} \rightarrow 3 \mathrm{FeO}+\frac{1}{2} \mathrm{O}_{2} \\
\mathrm{H}_{2} \mathrm{O}+3 \mathrm{FeO} \rightarrow \mathrm{Fe}_{3} \mathrm{O}_{4}+\mathrm{H}_{2}
\end{gathered}
$$

Among them, metal $\mathrm{Zn}$ is easy to form a dense oxide film, which is wrapped on the metal surface to prevent the reaction from proceeding. Wegner et al. [23] designed a spray reactor for solving this problem. By increasing the specific surface area of metal $\mathrm{Zn}$ to increase the contact area in the reaction, the experiment proves that the chemical conversion of $\mathrm{Zn}$ can reach $83 \%$. The disadvantage of this method is that the metal $\mathrm{Zn}$ needs to be gasified and atomized, which requires large energy consumption; $\mathrm{Zn}$, Sn, and other metals are also easily oxidized again during the decomposition process, affecting the reaction efficiency. The oxidation rate of iron oxide is easily reduced due to sintering, and ferrite has strong reducing ability. It can reduce $\mathrm{CO}_{2}$ to $\mathrm{C}$ solid element and cover the surface of ferrite to prevent the reaction from proceeding. One of the materials currently considered to be the most suitable for the thermochemical cycle of metal oxides is cerium oxide $\left(\mathrm{CeO}_{2}\right)$, because cerium oxide can efficiently reduce water or carbon dioxide to hydrogen or carbon monoxide [24], and cerium oxide also has good anti-coking properties. The specific reaction equations are:

High temperature (reduction step):

$$
\frac{1}{\delta_{r e d}-\delta_{o x}} \mathrm{CeO}_{2-\delta_{o x}} \rightarrow \frac{1}{\delta_{\text {red }}-\delta_{o x}} \mathrm{CeO}_{2-\delta_{\text {red }}}+\frac{1}{2} \mathrm{O}_{2}
$$

Low temperature (oxidation step):

$$
\mathrm{H}_{2} \mathrm{O}+\frac{1}{\delta_{r e d}-\delta_{o x}} \mathrm{CeO}_{2-\delta_{\text {red }}} \rightarrow \frac{1}{\delta_{\text {red }}-\delta_{o x}} \mathrm{CeO}_{2-\delta_{o x}}+\mathrm{H}_{2}
$$

In the two-step thermochemical cycle hydrogen production process, because there is a large heat transfer temperature difference between the "oxidation step" and "reduction step" (e.g., the temperature difference between cerium oxide heat transfer is about $700^{\circ} \mathrm{C}$ ), the thermal energy recovery of solid materials has always been a very difficult problem. Hao et al. [25] proposed an "isothermal" thermochemical cycle, that is, the "oxidation step" and "reduction step" reactions are performed at the same temperature. The "isothermal" thermochemical cycle effectively overcomes the defect that a large amount of solid sensible heat in the "dual-temperature" thermochemical cycle cannot be efficiently recovered and does not generate thermal stress, which can maintain high energy utilization efficiency at high temperatures. However, the isothermal thermochemical cycle also has certain limitations that need to be resolved, such as the requirement of maintaining a quite low oxygen partial pressure, less hydrogen production in a single cycle, etc. The thermochemical cycle with metal oxide can also be utilized for $\mathrm{CO}$ generation from $\mathrm{CO}_{2}$, and the thermodynamics is similar to that of $\mathrm{H}_{2}$ generation from $\mathrm{H}_{2} \mathrm{O}$, which will not be discussed here. 


\section{Carbon feed-based solar thermochemistry}

\subsection{Methane reforming and decomposition}

Nowadays, more than $95 \%$ of the hydrogen for refinery use is produced via hydrocarbon steam reforming [26]. Industrial hydrogen production through methane steam reforming exceeds 50 million tons annually and accounts for $2-5 \%$ of global energy consumption [27]. Methane steam reforming process for hydrogen production is usually described by the following reactions:

$$
\begin{gathered}
\mathrm{CH}_{4}(\mathrm{~g})+\mathrm{H}_{2} \mathrm{O}(\mathrm{g}) \rightleftharpoons \mathrm{CO}(\mathrm{g})+3 \mathrm{H}_{2}(\mathrm{~g}), \Delta{H^{\circ}}_{25^{\circ} \mathrm{C}}=205.9 \mathrm{~kJ} / \mathrm{mol} \\
\mathrm{CO}(\mathrm{g})+\mathrm{H}_{2} \mathrm{O}(\mathrm{g}) \rightleftharpoons \mathrm{CO}_{2}(\mathrm{~g})+\mathrm{H}_{2}(\mathrm{~g}), \Delta H^{\circ}{ }_{25^{\circ} \mathrm{C}}=-41.1 \mathrm{~kJ} / \mathrm{mol}
\end{gathered}
$$

where the reversible water-gas shift reaction Eq. (11) is sometimes considered as superimposed onto the methane reforming reaction Eq. (10) for conveniences of analysis on methane conversion:

$$
\mathrm{CH}_{4}(\mathrm{~g})+2 \mathrm{H}_{2} \mathrm{O}(\mathrm{g}) \rightleftharpoons \mathrm{CO}_{2}(\mathrm{~g})+4 \mathrm{H}_{2}(\mathrm{~g}), \Delta{H^{\circ}}^{\circ}{ }^{\circ} \mathrm{C}=164.8 \mathrm{~kJ} / \mathrm{mol}
$$

The methane dry reforming is the reaction between methane and carbon dioxide for syngas generation, given as:

$$
\mathrm{CH}_{4}(\mathrm{~g})+\mathrm{CO}_{2}(\mathrm{~g}) \rightleftharpoons 2 \mathrm{CO}(\mathrm{g})+2 \mathrm{H}_{2}(\mathrm{~g}), \Delta H^{\circ}{ }_{25^{\circ} \mathrm{C}}=247.0 \mathrm{~kJ} / \mathrm{mol}
$$

The reforming reactions, Eqs. (10), (12), and (13), are highly endothermic, and a large amount of heat is often provided by burning a supplemental amount of methane [28], which will decrease the heat value of fuel gas generated by $22 \%$ for the same amount of methane consumed and release large amounts of greenhouse gas $\mathrm{CO}_{2}$ [29]. In recent years, as the technologies of concentrated solar energy (CSE) and solar thermal utilization improve rapidly, methane reforming driven by CSE emerged as a promising method for hydrogen production [30], which derives heat from solar energy instead of fossil fuels. Besides, solar thermal energy with relatively low temperatures (compared with methane combustion) is absorbed by methane reforming reaction and upgraded to the chemical energy with higher energy level (ratio of exergy change $\Delta E$ to enthalpy change $\Delta H$ of a process [31]) in such process. Solar energy thus stored in hydrogen as chemical energy, and it could be converted into power with significantly greater efficiencies than that of solarthermal-only power generation in the same temperature range [32].

In order to achieve the high-efficient progress of the solar methane reforming reaction, research on solar methane reforming reactors has also continued. Klein et al. [33] proposed a schematic diagram of a fluidized bed reactor. This experiment is a methane dry reforming experiment, where gas reactants and carbon particles are mixed and passed into the reactor together. The reactor can achieve a concentration ratio of 3000 through primary and secondary light concentration, and the reaction temperature is between 950 and $1450^{\circ} \mathrm{C}$ with the ratio of carbon dioxide to methane changes from 1: 1 to $6: 1$, which has a maximum methane conversion of $90 \%$. Edwards et al. [34] studied methane steam reforming in a solar tubular reactor, which is condensed by a $107 \mathrm{~m}^{2}$ dish condenser. The condensing temperature can reach $850^{\circ} \mathrm{C}$, and the pressure can reach 20 bars. The reactor can stably produce hydrogen, but there is no detailed introduction on the conversion rate in the literature. The device for hydrogen production by metal oxide thermochemical cycling was proposed by Steinfeld et al. [35]. The system contains a $51.8 \mathrm{~m}^{2}$ heliostat to focus the sunlight at first, and then the sunlight passed through a parabolic surface with an opening area of $2.7 \mathrm{~m}^{2}$ to focus it again. The final focusing ratio was 


\begin{tabular}{|c|c|c|c|c|c|c|c|}
\hline Participant & $\begin{array}{l}\mathrm{Q}_{\text {solar }} \\
(\mathrm{kW})\end{array}$ & $\begin{array}{l}\text { Pressure } \\
\quad(\text { bar })\end{array}$ & $\begin{array}{c}T \\
(\mathrm{~K})\end{array}$ & $\begin{array}{c}\text { Reactor } \\
\text { type }\end{array}$ & $\begin{array}{c}\text { Reactant conversion } \\
(\%)\end{array}$ & $\begin{array}{l}\text { Efficiency } \\
(\%)\end{array}$ & Ref. \\
\hline PSI & 5 & $>1$ & 1600 & $\begin{array}{l}\text { Vortex } \\
\text { flow }\end{array}$ & 64 & $15.1^{\mathrm{a}}, 16.2^{\mathrm{b}}$ & [37] \\
\hline PSI & 5 & $>1$ & 1600 & $\begin{array}{l}\text { Particle } \\
\text { flow }\end{array}$ & 99 & $16.1^{\mathrm{b}}$ & [38] \\
\hline $\begin{array}{l}\text { PROMES- } \\
\text { CNRS }\end{array}$ & 10 & 0.4 & 1773 & Tubular & 98 & $4.8^{\mathrm{b}}$ & {$[39,40]$} \\
\hline $\begin{array}{l}\text { PROMES- } \\
\text { CNRS }\end{array}$ & 50 & 0.45 & 1928 & Tubular & 100 & $13.5^{\mathrm{a}}, 15.2^{\mathrm{b}}$ & [41] \\
\hline NREL & 6 & 1 & 2133 & $\begin{array}{l}\text { Aerosol } \\
\text { flow }\end{array}$ & 90 & $2^{b}$ & [42] \\
\hline $\begin{array}{l}\text { PROMES- } \\
\text { CNRS }\end{array}$ & 0.8 & 0.61 & 1700 & $\begin{array}{l}\text { Nozzle } \\
\text { type }\end{array}$ & 95 & $5.9^{\mathrm{a}}$ & [43] \\
\hline
\end{tabular}

Table 2.

Summary of experimental research on solar methane decomposition [36].

3500. During the reaction, $\mathrm{ZnO}$ particles with an average particle diameter of $0.4 \mu \mathrm{m}$ were sent into a cylindrical reaction chamber for reaction by methane. The products were $\mathrm{Zn}$ simple substance and synthesis gas $\left(\mathrm{H}_{2}, \mathrm{CO}\right)$. The reactor can reach $50 \%$ methane conversion at $1030^{\circ} \mathrm{C}$.

Methane decomposition is also an endothermic reaction at $600-1200^{\circ} \mathrm{C}$, given as:

$$
\mathrm{CH}_{4}(\mathrm{~g}) \rightleftharpoons \mathrm{C}+2 \mathrm{H}_{2}(\mathrm{~g}), \Delta H^{\circ}{ }_{25^{\circ} \mathrm{C}}=74.6 \mathrm{~kJ} / \mathrm{mol}
$$

Solar methane decomposition has been researched in both indirectly and directly heated reactors from solar thermal energy. A summary of experimental study has been listed in Table 2.

\subsection{Methanol reforming and decomposition}

Methanol reforming and decomposition also attracts lots of attention in the field of solar thermochemical fuel generation [44], as the reaction temperature is about $150-300^{\circ} \mathrm{C}$, which is quite low and easy to be maintained by line-focusing solar collector (parabolic trough collector or linear Fresnel lens) with low cost. The reaction equations of methanol reforming and decomposition are given as:

$$
\begin{gathered}
\mathrm{CH}_{3} \mathrm{OH}(\mathrm{g})+\mathrm{H}_{2} \mathrm{O}(\mathrm{g}) \rightarrow \mathrm{CO}_{2}(\mathrm{~g})+3 \mathrm{H}_{2}(\mathrm{~g}), \Delta H^{\circ}{ }_{25^{\circ} \mathrm{C}}=49.321 \mathrm{~kJ} / \mathrm{mol} \\
\mathrm{CH}_{3} \mathrm{OH}(\mathrm{g}) \rightarrow \mathrm{CO}(\mathrm{g})+2 \mathrm{H}_{2}(\mathrm{~g}), \Delta H^{\circ}{ }_{25^{\circ} \mathrm{C}}=90.459 \mathrm{~kJ} / \mathrm{mol}
\end{gathered}
$$

Both of the two reactions are endothermic, which can convert the low-level solar thermal energy (low temperature) into high-level chemical energy, and have been researched with combining with other systems, like PV cell module, and combined cooling heating and power in downstream.

\subsection{Biomass gasification}

Biomass is widespread and is often perceived as a carbon-neutral source of energy. Solar biomass gasification is a clean route to obtain fuels, which may also reach liquid fuel for vehicle or jet utilization. Detailed reviews on solar biomass 


\begin{tabular}{|c|c|c|c|c|c|c|c|c|}
\hline Feed & $\begin{array}{l}Q_{\text {solar }} \\
(k W)\end{array}$ & $\begin{array}{c}T \\
(\mathrm{~K})\end{array}$ & $\begin{array}{l}\text { Gasifying } \\
\text { agent }\end{array}$ & $\begin{array}{l}\text { Reactor } \\
\text { type }\end{array}$ & $\begin{array}{c}\text { Reactant } \\
\text { conversion } \\
(\%)\end{array}$ & $\begin{array}{l}\text { Product } \\
\text { yield } \\
(\%)\end{array}$ & $\begin{array}{c}\text { Efficiency } \\
(\%)\end{array}$ & Ref. \\
\hline $\begin{array}{l}\text { Bituminous } \\
\text { coal }\end{array}$ & 1.2 & 1600 & $\mathrm{CO}_{2}$ & $\begin{array}{l}\text { Fluidized } \\
\text { bed quartz } \\
\text { tubular } \\
\text { reactor }\end{array}$ & 65 & - & $8^{\mathrm{a}}$ & [49] \\
\hline Pet coke & 5 & 1818 & Steam & Vortex flow & 87 & $\begin{array}{l}35\left(\mathrm{H}_{2}\right) \\
15(\mathrm{CO})\end{array}$ & $9^{a}, 20^{b}$ & {$[50]$} \\
\hline $\begin{array}{l}\text { Pet coke- } \\
\text { water slurry }\end{array}$ & 5 & 1500 & Steam & Vortex flow & 87 & $\begin{array}{l}65\left(\mathrm{H}_{2}\right) \\
25(\mathrm{CO})\end{array}$ & $4.7^{\mathrm{a}}, 17.4^{\mathrm{b}}$ & {$[51]$} \\
\hline $\begin{array}{l}\text { Petroleum } \\
\text { VR }\end{array}$ & 5 & 1573 & Steam & Vortex flow & 50 & $\begin{array}{l}68\left(\mathrm{H}_{2}\right) \\
15(\mathrm{CO})\end{array}$ & $2^{c}, 19^{a}$ & {$[52]$} \\
\hline Coal coke & 0.94 & 1123 & $\mathrm{CO}_{2}$ & $\begin{array}{l}\text { Internally } \\
\text { circulating } \\
\text { fluidized bed }\end{array}$ & - & - & $12^{\mathrm{a}}$ & [53] \\
\hline $\begin{array}{l}\text { IS, SS, STP, } \\
\text { fluff, SAC, } \\
\text { beech } \\
\text { charcoal }\end{array}$ & 5 & 1490 & Steam & Packed bed & 100 & $\begin{array}{c}\mathrm{H}_{2} / \\
\mathrm{CO}=1.5 \\
\mathrm{CO}_{2} / \\
\mathrm{CO}=0.2\end{array}$ & $\begin{array}{c}29^{\mathrm{d}} \\
U=1.3^{\mathrm{e}}\end{array}$ & [54] \\
\hline $\begin{array}{l}\text { Beech } \\
\text { charcoal }\end{array}$ & 3 & 1523 & Steam & $\begin{array}{l}\text { Particle flow } \\
\text { reactor }\end{array}$ & 30 & - & $1.53^{\mathrm{b}}$ & [55] \\
\hline Coal coke & 1.1 & 1573 & $\mathrm{CO}_{2}$ & $\begin{array}{l}\text { Fluidized } \\
\text { bed }\end{array}$ & 42 & - & $14^{\mathrm{a}}$ & {$[56]$} \\
\hline Coal coke & 3 & 1773 & $\mathrm{CO}_{2}$ & $\begin{array}{l}\text { Internally } \\
\text { circulating } \\
\text { fluidized bed }\end{array}$ & 73 & - & $12^{\mathrm{f}}$ & {$[57]$} \\
\hline $\begin{array}{l}\text { LRK, tire } \\
\text { chips, Fluff, } \\
\text { DSS, IS, SB }\end{array}$ & 150 & $\begin{array}{c}1350- \\
1453\end{array}$ & Steam & Packed bed & $36-100$ & $\begin{array}{c}\mathrm{H}_{2} / \\
\mathrm{CO}=2- \\
5.2\end{array}$ & $\begin{array}{c}25-35^{\mathrm{d}}, \\
U=1.03- \\
1.30^{\mathrm{e}}\end{array}$ & [58] \\
\hline \multicolumn{9}{|c|}{$\begin{array}{l}{ }^{a} \eta_{\text {chemical }}=\frac{\dot{Q}_{\text {chem }}}{\dot{Q}_{\text {solar }}} \\
{ }^{b} \eta_{\text {thermal }}=\frac{\dot{Q}_{\text {chem }}+\dot{Q}_{\text {sensible }}}{Q_{\text {solar }}+Q_{\text {steam }}} . \\
{ }^{c} \eta=\dot{n}_{\mathrm{H}_{2} L H V_{\mathrm{H}_{2}}+\dot{n}_{\mathrm{CO}} L H V_{\mathrm{CO}}+\sum_{i}^{\text {spceies }} \int_{473 K}^{T_{\text {reactor }}} \dot{n}_{i} C_{p, i}(T) \mathrm{d} T} .\end{array}$} \\
\hline
\end{tabular}

Table 3.

Summary of experimental research published in gasification of solid hydrocarbon feed [36].

gasification have been conducted by Epstein et al. [45], Lédé [46], Nzihou et al. [47], and Puig-Arnavat et al. [48], which will not be discussed here. A summary of experimental work published in gasification of solid hydrocarbon feed has been listed in Table 3.

\section{Solar thermochemical fuel generation by membrane reactor}

Solar thermochemistry usually requires high temperature (e.g., above $4000^{\circ} \mathrm{C}$ for $\mathrm{H}_{2} \mathrm{O}$ splitting; $3000^{\circ} \mathrm{C}$ for $\mathrm{CO}_{2}$ splitting; $700-1000^{\circ} \mathrm{C}$ for methane reforming), 
which requires high concentration ratio and large mirror area, and the system will be more complex and expensive. In situ separation by a permeable membrane for a target product shifts thermodynamic equilibrium of chemical reactions in favor of reactants conversion, which equivalently lowers solar collection temperature. Combination of membrane reactor and solar thermal collection offers unique advantages in many respects, such as the increment of conversion rate, decrease of reaction temperature, and emission reduction, which are otherwise unattainable by either alone. Besides, the all-solid-state feature and isothermal operation enable compact design of solar fuel reactors with minimized thermal stress. Now, the selective permeation membrane for gas species in high temperature is mainly oxygen permeation membrane, hydrogen permeation membrane, and carbon dioxide permeation membrane, which have been researched for solar thermochemical fuel generation.

\subsection{Oxygen permeation membrane for $\mathrm{H}_{2} \mathrm{O} / \mathrm{CO}_{2}$ splitting}

Perovskites, $\mathrm{ZrO}_{2}$ and $\mathrm{CeO}_{2}$ (or doped $\mathrm{ZrO}_{2}$ and $\mathrm{CeO}_{2}$ ), usually constitute the selective oxygen permeation membrane utilized in high temperature $\left(>600^{\circ} \mathrm{C}\right)$. Wang et al. [59] proposed a theoretical framework for the thermodynamic analysis of solar oxygen permeation membrane reactor, and the solar-to-fuel efficiency (ratio of the higher heating value of products to the total energy input) can reach as high as $89 \%$ in methane-assisted membrane reactor. Zhu et al. [60] brought up a thermodynamic model of ceria dense membrane for $\mathrm{CO}_{2}$ splitting, and the energy efficiency is above $10 \%$ at $1800 \mathrm{~K}$ without heat recovery. Steinfeld et al. [61, 62] have done a lot of experimental researches about solar $\mathrm{CO}_{2}$ splitting for $\mathrm{CO}$ generation by oxygen permeation membrane with $100 \%$ selectivity (e.g., $\mathrm{La}_{0.6} \mathrm{Sr}_{0.4} \mathrm{Co}_{0.2}$ $\mathrm{Fe}_{0.8} \mathrm{O}_{3-\delta}$ at $1030^{\circ} \mathrm{C}[61], \mathrm{CeO}_{2}$ at $1600^{\circ} \mathrm{C}$ [62]), and Ozin [63] said the research of Steinfeld is an elegant demonstration and an exciting breakthrough for continuous $\mathrm{CO}_{2}$ splitting in a single step, at a single temperature, in a single reactor.

\subsection{Hydrogen permeation membrane for hydrogen generation}

The materials of the hydrogen permeation membrane are various, such as metal (e.g., palladium, nickel), perovskites, pyrochlores, fluorites, polymers, which are usually used in the reaction of reforming, splitting, partial oxidation of hydrocarbon, splitting of other hydrogen carriers (e.g., $\mathrm{NH}_{3}$ ), and water-gas shift reaction. $\mathrm{Li}$ et al. [30] first presented an innovative solar-assisted hybrid power system integrated with methane steam reforming in membrane reactor, and the simulation results showed that capture ratio of $\mathrm{CO}_{2}$ is $91 \%$ and exergy efficiency and thermal efficiency are 58 and $51.6 \%$ (10.2 and 2.2\% points higher than the $\mathrm{CO}_{2}$ capture from exhaust cycle), respectively. Said et al. [64] simulated a CFD model about solar molten salt-heated $\mathrm{H}_{2}$-selective membrane reformer for methane upgrading and hydrogen generation, and the results showed the fuel heating value upgrade of $40 \%$ with methane conversion rate of $99 \%$ and hydrogen recovery of $87 \%$ at $600^{\circ} \mathrm{C}$. Wang et al. [65] put forward a novel reactor, which realized direct methane steam reforming in parabolic trough collector integrated with hydrogen permeation membrane reactor, and the system can perform high and stable efficiency (above $80 \%)$ at $400^{\circ} \mathrm{C}$. Mallapragada et al. [66] proposed a novel system that consists of oxygen permeation membrane and hydrogen permeation membrane for solar water splitting, and the solar-to- $\mathrm{H}_{2}$ efficiency (ratio of the lower heating value of hydrogen to the reversible work input for Gibbs free energy change of water splitting) is $72.4-80.1 \%$ at the concentration ratios of 2000-10,000. Sui et al. [67] reported an exploration on an efficient solar thermochemical water-splitting system enhanced 
by hydrogen permeation membrane, which has showed a sharply enhanced conversion rate of $87.8 \%$ at $1500^{\circ} \mathrm{C}$ and $10^{-5}$ bar at permeated side (versus $1.26 \%$ with oxygen permeation membrane or isothermal thermochemical cycle). Recently, a promising method for hydrogen generation without carbon emitting by ammonia decomposition in a catalytic palladium membrane reactor for hydrogen separation driven by solar energy has been theoretically proposed, and the first-law thermodynamic efficiency, net solar-to-hydrogen efficiency, and exergy efficiency can reach as high as $86.86,40.08$, and $72.07 \%$, respectively [68].

\subsection{Carbon dioxide permeation membrane for hydrocarbon reforming}

Carbon dioxide permeation membrane includes mixed $\mathrm{e}^{-} / \mathrm{CO}_{3}{ }^{2-}$ conducting membrane, $\mathrm{O}^{2-} / \mathrm{CO}_{3}{ }^{2-}$ conducting membrane, $\mathrm{OH}^{-} / \mathrm{CO}_{3}{ }^{2-}$ conducting membrane (hydroxide/ceramic dual-phase membrane), etc. [69, 70]. The combination between carbon dioxide permeation membrane and solar energy is very limited now. The combination of hydrogen permeation membrane and carbon dioxide permeation membrane has been proposed for methane steam reforming by way of an alternate $\mathrm{H}_{2}$ and $\mathrm{CO}_{2}$ separation driven by solar energy [71]. The carbon dioxide permeation membrane has great potential to be utilized for the hydrocarbon reforming or decomposition for $\mathrm{CO}_{2}$ separation and capture in the future.

\subsection{Challenges and perspectives}

Though the solar membrane reactor has lots of advantages and immense potential for application mentioned above, the efficient approach to lower the partial pressure of gas product (or avoid the relatively low pressure) is the main challenge to maintain a high energy conversion rate, and the improvement of stability and permeability of membrane material at corresponding reaction temperature is also significant. These issues have potential areas for big breakthroughs and require further studies to address. The multiple product separation with membrane reactor may be a promising method to increase the energy efficiency, due to a relatively high partial pressure and less separation energy required [71].

\section{Conclusions}

This chapter has reviewed the state-of-the-art researches about solar thermochemical fuel generation, and the highlighted conclusions are listed:

a. The thermodynamics in solar thermochemical fuel generation has been analyzed, and the maximum theoretical efficiency from solar energy to work has been obtained.

b. The most representative solar thermochemical reactions (e.g., $\mathrm{H}_{2} \mathrm{O} / \mathrm{CO}_{2}$ splitting, hydrocarbon reforming, and decomposition) have been reviewed, and the advantages and drawbacks have been analyzed and discussed.

c. Thermochemical cycle and membrane reactor driven by solar energy have been systematically introduced, which could decrease the reaction temperature and have the potential to be widely utilized in the future, especially the membrane reactor, which could purify the product with a continuous operation. 


\section{Acknowledgements}

This work is funded by the National Natural Science Foundation of China (no. 51906179) and the State Scholarship Fund (No. 201906275035) from China Scholarship Council.

\section{Author details}

Hongsheng Wang ${ }^{1,2}$

1 Wuhan University, Wuhan, China

2 The University of Tokyo, Tokyo, Japan

*Address all correspondence to: wanghongsheng@whu.edu.cn

\section{IntechOpen}

(C) 2020 The Author(s). Licensee IntechOpen. This chapter is distributed under the terms of the Creative Commons Attribution License (http://creativecommons.org/licenses/ by/3.0), which permits unrestricted use, distribution, and reproduction in any medium, provided the original work is properly cited. (c) BY 


\section{References}

[1] Kannan N, Vakeesan D. Solar energy for future world: a review. Renewable and Sustainable Energy Reviews. 2016; 62:1092-1105. DOI: 10.1016/j.rser. 2016.05.022

[2] Simakov DSA, Wright MM, Ahmed S, Mokheimer EMA, RománLeshkov Y. Solar thermal catalytic reforming of natural gas: A review on chemistry, catalysis and system design. Catalysis Science \& Technology. 2015;5: 1991-2016. DOI: 10.1039/C4CY01333F

[3] Kong H, Hao Y, Wang H. A solar thermochemical fuel production system integrated with fossil fuel heat recuperation. Applied Thermal Engineering. 2016;108:958-966. DOI: 10.1016/j.applthermaleng.2016.03.170

[4] Kong H, Kong X, Wang H, Wang J. A strategy for optimizing efficiencies of solar thermochemical fuel production based on nonstoichiometric oxides. International Journal of Hydrogen Energy. 2019;44(36):19585-19594. DOI: 10.1016/j.ijhydene.2019.05.197

[5] Fletcher EA. On the thermodynamics of solar energy use. Journal of the Minnesota Academy of Science. 1983; 49:30-34

[6] Wang H, Li W, Liu T, Liu X, Hu X. Thermodynamic analysis and optimization of photovoltaic/thermal hybrid hydrogen generation system based on complementary combination of photovoltaic cells and proton exchange membrane electrolyzer. Energy Conversion and Management. 2019;183:97-108. DOI: 10.1016/j. enconman.2018.12.106

[7] Yilanci A, Dincer I, Ozturk HK. A review on solar-hydrogen/fuel cell hybrid energy systems for stationary applications. Progress in Energy and Combustion Science. 2009;35(3):231-244. DOI: 10.1016/j.pecs.2008. 07.004
[8] Lovegrove K, Wes S. Concentrating solar power technology: Principles, developments and applications. Cambridge, UK: Elsevier; 2012

[9] IRENA. Renewable Energy Cost Analysis-Concentrating Solar Power. Abu Dhabi, United Arab Emirates: International Renewable Energy Agency; 2012

[10] IRENA. Renewable Power Generation Costs in 2014. Abu Dhabi, United Arab Emirates: International Renewable Energy Agency; 2014

[11] SunShot. SunShot Vision Study. Golden, USA: U.S. Department of Energy; 2012

[12] Ayre J. New solar stirling dish efficiency record of 32\% set. Clean Technica. 2013;17. Available from: https://cleantechnica.com/2013/01/17/ new-solar-stirling-dish-efficiencyrecord-of-32-set/

[13] Siva Reddy V, Kaushik SC, Ranjan KR, Tyagi SK. State of-the-art of solar thermal power plants-A review. Renewable and Sustainable Energy Reviews. 2013;27:258-273. DOI: 10.1016/ j.rser.2013.06.037

[14] Pavlović TM, Radonjić IS, Milosavljević DD, Pantić LS. A review of concentrating solar power plants in the world and their potential use in Serbia. Renewable and Sustainable Energy Reviews. 2012;16(6):3891-3902. DOI: 10.1016/j.rser.2012.03.042

[15] Kaygusuz K. Prospect of concentrating solar power in Turkey: The sustainable future. Renewable and Sustainable Energy Reviews. 2011;15(1): 808-814. DOI: 10.1016/j.rser.2010. 09.042

[16] Weinstein LA, Loomis J, Bhatia B, Bierman DM, Wang EN, Chen G. Concentrating solar power. Chemical 
Reviews. 2015;115(23):12797-12838.

DOI: 10.1021/acs.chemrev.5b00397

[17] Steinfeld A, Palumbo R. Solar thermochemical process technology.

Encyclopedia of Physical Science and Technology. 2001;15(1):237-256

[18] Fletcher EA, Moen RL. Hydrogen and oxygen from water. Science. 1977; 197(4308):1050-1056. DOI: $10.1126 /$ science.197.4308.1050

[19] Zhang P, Yu B, Chen J, Xu J. Study on the hydrogen production by thermochemical water splitting. Progress in. Chemistry. 2005;17(4): 643-650

[20] Spewock S, Brecher LE, Talko F. The thermal catalytic decomposition of sulfur trioxide to sulfur dioxide and oxygen. In: Proceedings of the 1st World Hydrogen Energy Conference. Coral Gables, FL: University of Miami Press. p. 1976

[21] Sakurai M, Bilgen E, Tsutsumi A, Yoshida K. Adiabatic UT-3 thermochemical process for hydrogen production. International Journal of Hydrogen Energy. 1996;21(10):865-870. DOI: 10.1016/0360-3199(96)00024-9

[22] Doctor RD, Dacid CW, Mendelsohn $\mathrm{MH}$. AIChE Spring National Meeting; New Orleans, 2002.

[23] Wegner K, Ly HC, Weiss RJ, Pratsinis SE, Steinfeld A. In situ formation and hydrolysis of $\mathrm{Zn}$ nanoparticles for $\mathrm{H}_{2}$ production by the 2-step $\mathrm{ZnO} / \mathrm{Zn}$ water-splitting thermochemical cycle. International Journal of Hydrogen Energy. 2006; 31(1):55-61. DOI: 10.1016/j. ijhydene.2005.03.006

[24] Chueh WC, Falter C, Abbott M, Scipio D, Furler P, Haile SM, et al. Highflux solar-driven thermochemical dissociation of $\mathrm{CO}_{2}$ and $\mathrm{H}_{2} \mathrm{O}$ using nonstoichiometric ceria. Science. 2010;
330:1797-1801. DOI: 10.1126/

science. 1197834

[25] Hao Y, Yang CK, Haile SM. Hightemperature isothermal chemical cycling for solar-driven fuel production. Physical Chemistry Chemical Physics. 2013;15(40):17084-17092

[26] Collodi G, Wheeler F. Hydrogen production via steam reforming with $\mathrm{CO}_{2}$ capture. Chemical Engineering Transactions. 2010;19:37-42

[27] Saavedra J, Whittaker T, Chen Z, Pursell CJ, Rioux RM, Chandler BD. Controlling activity and selectivity using water in the Au-catalysed preferential oxidation of $\mathrm{CO}$ in $\mathrm{H}_{2}$. Nature Chemistry. 2016;8:584-589. DOI: 10.1038/nchem.2494

[28] Barelli L, Bidini G, Gallorini F, Servili S. Hydrogen production through sorption-enhanced steam methane reforming and membrane technology: A review. Energy. 2008;33(4):554-570.

DOI: 10.1016/j.energy.2007.10.018

[29] Fan J, Zhu L. Performance analysis of a feasible technology for power and high-purity hydrogen production driven by methane fuel. Applied Thermal Engineering. 2015;75:103-114. DOI: 10.1016/j.applthermaleng.2014.10.013

[30] Li Y, Zhang N, Cai R. Low $\mathrm{CO}_{2}-$ emissions hybrid solar combined-cycle power system with methane membrane reforming. Energy. 2013;58:36-44. DOI: 10.1016/j.energy.2013.02.005

[31] Ishida M, Kawamura K. Energy and exergy analysis of a chemical process system with distributed parameters based on the enthalpy-direction factor diagram. Industrial \& Engineering Chemistry Process Design and Development. 1982;21(4):690-695

[32] Han W, Jin H, Zhang N, Zhang X. Cascade utilization of chemical energy of natural gas in an improved CRGT 
cycle. Energy. 2007;32(4):306-313. DOI: 10.1016/j.energy.2006.06.014

[33] Klein HH, Karni J, Rubin R. Dry methane reforming without a metal catalyst in a directly irradiated solar particle reactor. Journal of Solar Energy Engineering. 2009;131(2):021001-1021001-14. DOI: 10.1115/1.3090823

[34] Edwards JH, Duffy GJ, Benito R, Do T, Dave N, McNaughton R, et al. CSIRO's solar thermal-fossil energy hybrid technology for advanced power generation. In: Proceedings of Solar Thermal 2000 10th SolarPACES International Symposium on Solar Thermal Concentrating Technologies; Sydney, N.S.W. 2000. pp. 27-32

[35] Steinfeld A, Brack M, Meier A, Weidenkaff A, Wuillemin D. A solar chemical reactor for co-production of zinc and synthesis gas. Energy. 1998; 23(10):803-814. DOI: 10.1016/ S0360-5442(98)00026-7

[36] Yadav D, Banerjee R. A review of solar thermochemical processes. Renewable and Sustainable Energy Reviews. 2016;54:497-532. DOI: 10.1016/j.rser.2015.10.026

[37] Hirsch D, Steinfeld A. Solar hydrogen production by thermal decomposition of natural gas using a vortex-flow reactor. International Journal of Hydrogen Energy. 2004; 29(1):47-55. DOI: 10.1016/S0360-3199 (03) 00048-X

[38] Maag G, Zanganeh G, Steinfeld A. Solar thermal cracking of methane in a particle-flow reactor for the coproduction of hydrogen and carbon. International Journal of Hydrogen Energy. 2009;34(18):7676-7685. DOI: 10.1016/j.ijhydene.2009.07.037

[39] Rodat S, Abanades S, Sans JL, Flamant G. Hydrogen production from solar thermal dissociation of natural gas: Development of a $10 \mathrm{~kW}$ solar chemical reactor prototype. Solar Energy. 2009; 83(9):1599-1610. DOI: 10.1016/j. solener.2009.05.010

[40] Maag G, Rodat S, Flamant G, Steinfeld A. Heat transfer model and scale-up of an entrained-flow solar reactor for the thermal decomposition of methane. International Journal of Hydrogen Energy. 2010;35(24): 13232-13241. DOI: $10.1016 / j$. ijhydene.2010.08.119

[41] Rodat S, Abanades S, Sans JL, Flamant G. A pilot-scale solar reactor for the production of hydrogen and carbon black from methane splitting. International Journal of Hydrogen Energy. 2010;35(15):7748-7758. DOI: 10.1016/j.ijhydene.2010.05.057

[42] Dahl JK, Buechler KJ, Weimer AW, Lewandowski A, Bingham C. Solarthermal dissociation of methane in a fluid-wall aerosol flow reactor. International Journal of Hydrogen Energy. 2004;29(7):725-736. DOI: 10.1016/j.ijhydene.2003.08.009

[43] Abanades S, Flamant G. Production of hydrogen by thermal methane splitting in a nozzle-type laboratoryscale solar reactor. International Journal of Hydrogen Energy. 2005;30(8): 843-853. DOI: 10.1016/j.ijhydene.2004. 09.006

[44] Liu Q, Hong H, Yuan J, Jin H, Cai R. Experimental investigation of hydrogen production integrated methanol steam reforming with middle-temperature solar thermal energy. Applied Energy. 2009;86(2):155-162. DOI: 10.1016/j. apenergy.2008.03.006

[45] Epstein M, Spiewak I, Funken KH, Ortner J. Review of the technology for solar gasification of carbonaceous materials. Solar Engineering. 1994; 26(13):79-91

[46] Lédé J. Solar thermochemical conversion of biomass. Solar Energy. 
1999;65(1):3-13. DOI: $10.1016 /$

S0038-092X(98)00109-1

[47] Nzihou A, Flamant G, Stanmore B. Synthetic fuels from biomass using concentrated solar energy-A review. Energy. 2012;42(1):121-131. DOI: 10.1016/j.energy.2012.03.077

[48] Puig-Arnavat M, Tora EA, Bruno JC, Coronas A. State of the art on reactor designs for solar gasification of carbonaceous feedstock. Solar Energy. 2013;97:67-84. DOI: 10.1016/j. solener.2013.08.001

[49] Kodama T, Kondoh Y, Tamagawa T, Funatoh A, Shimizu KI, Kitayama Y. Fluidized bed coal gasification with $\mathrm{CO}_{2}$ under direct irradiation with concentrated visible light. Energy \& Fuels. 2002;16(5):1264-1270. DOI: 10.1021/ef020053x

[50] Z'Graggen A, Haueter P, Trommer D, Romero M, De Jesus JC, Steinfeld A. Hydrogen production by steam-gasification of petroleum coke using concentrated solar power-II. Reactor design, testing, and modeling. International Journal of Hydrogen Energy. 2006;31(6):797-811. DOI: 10.1016/j.ijhydene.2005.06.011

[51] Z'Graggen A, Haueter P, Maag G, Vidal A, Romero M, Steinfeld A. Hydrogen production by steamgasification of petroleum coke using concentrated solar power-III. Reactor experimentation with slurry feeding. International Journal of Hydrogen Energy. 2007;32(8):992-996. DOI: 10.1016/j.ijhydene.2006.10.001

[52] Z'Graggen A, Steinfeld A. Hydrogen production by steam-gasification of carbonaceous materials using concentrated solar energy-V. Reactor modeling, optimization, and scale-up. International Journal of Hydrogen Energy. 2008;33(20):5484-5492. DOI: 10.1016/j.ijhydene.2007.10.038
[53] Kodama T, Enomoto SI,

Hatamachi T, Gokon N. Application of an internally circulating fluidized bed for windowed solar chemical reactor with direct irradiation of reacting particles. Journal of Solar Energy Engineering. 2008;130(1):014504. DOI: $10.1115 / 1.2807213$

[54] Piatkowski N, Wieckert C, Steinfeld A. Experimental investigation of a packed-bed solar reactor for the steam-gasification of carbonaceous feedstocks. Fuel Processing Technology. 2009;90(3):360-366. DOI: 10.1016/j. fuproc.2008.10.007

[55] Melchior T, Perkins C, Lichty P, Weimer AW, Steinfeld A. Solar-driven biochar gasification in a particle-flow reactor. Chemical Engineering and Processing: Process Intensification. 2009;48(8):1279-1287. DOI: 10.1016/j. cep.2009.05.006

[56] Kodama T, Gokon N, Enomoto SI, Itoh S, Hatamachi T. Coal coke gasification in a windowed solar chemical reactor for beam-down optics. Journal of Solar Energy Engineering. 2010;132(4):041004. DOI: 10.1115/ 1.4002081

[57] Gokon N, Ono R, Hatamachi T, Liuyun L, Kim HJ, Kodama T. $\mathrm{CO}_{2}$ gasification of coal cokes using internally circulating fluidized bed reactor by concentrated Xe-light irradiation for solar gasification. International Journal of Hydrogen Energy. 2012;37(17):12128-12137. DOI: 10.1016/j.ijhydene.2012.05.133

[58] Wieckert C, Obrist A, Zedtwitz PV, Maag G, Steinfeld A. Syngas production by thermochemical gasification of carbonaceous waste materials in a 150 $\mathrm{kW}$ th packed-bed solar reactor. Energy \& Fuels. 2013;27(8):4770-4776. DOI: 10.1021/ef4008399

[59] Wang H, Hao Y, Kong H. Thermodynamic study on solar 
thermochemical fuel production with oxygen permeation membrane reactors. International Journal of Energy Research. 2015;39(13):1790-1799. DOI: 10.1002/er.3335

[60] Zhu L, Lu Y, Shen S. Solar fuel production at high temperatures using ceria as a dense membrane. Energy. 2016;104:53-63. DOI: 10.1016/j. energy.2016.03.108

[61] Michalsky R, Neuhaus D, Steinfeld A. Carbon dioxide reforming of methane using an isothermal redox membrane reactor. Energy Technology. 2015;3(7):784-789. DOI: 10.1002/ ente. 201500065

[62] Tou M, Michalsky R, Steinfeld A. Solar-driven thermochemical splitting of $\mathrm{CO}_{2}$ and in situ separation of $\mathrm{CO}$ and $\mathrm{O}_{2}$ across a ceria redox membrane reactor. Joule. 2017;1(1):146-154. DOI: 10.1016/j.joule.2017.07.015

[63] Ozin GA. “One-pot” solar fuels. Joule. 2017;1(1):19-23. DOI: 10.1016/j. joule.2017.08.010

[64] Said SA, Simakov DS, Waseeuddin M, Román-Leshkov Y. Solar molten salt heated membrane reformer for natural gas upgrading and hydrogen generation: A CFD model. Solar Energy. 2016;124:163-176. DOI: 10.1016/j.solener.2015.11.038

[65] Wang H, Liu M, Kong H, Hao Y. Thermodynamic analysis on $\mathrm{mid} /$ low temperature solar methane steam reforming with hydrogen permeation membrane reactors. Applied Thermal Engineering. 2019;152:925-936. DOI: 10.1016/j.applthermaleng.2018.03.030

[66] Mallapragada DS, Agrawal R. Limiting and achievable efficiencies for solar thermal hydrogen production. International Journal of Hydrogen Energy. 2014;39(1):62-75. DOI: 10.1016/ j.ijhydene.2013.10.075
[67] Sui C, Wang H, Liu X, Hu X. Solar thermochemical water-splitting reaction enhanced by hydrogen permeation membrane. arXiv preprint arXiv: 1808.02175. 2018

[68] Wang B, Kong H, Wang H, Wang Y, Hu X. Kinetic and thermodynamic analyses of mid/lowtemperature ammonia decomposition in solar-driven hydrogen permeation membrane reactor. International Journal of Hydrogen Energy. 2019;44(49): 26874-26887. DOI: 10.1016/j. ijhydene.2019.08.175

[69] Zhang L, Xu N, Li X, Wang S, Huang K, Harris WH, et al. High $\mathrm{CO}_{2}$ permeation flux enabled by highly interconnected three-dimensional ionic channels in selective $\mathrm{CO}_{2}$ separation membranes. Energy \& Environmental Science. 2012;5(8):8310-8317. DOI: 10.1039/C2EE22045H

[70] Ceron MR, Lai LS, Amiri A, Monte M, Katta S, Kelly JC, et al. Surpassing the conventional limitations of $\mathrm{CO}_{2}$ separation membranes with hydroxide/ceramic dual-phase membranes. Journal of Membrane Science. 2018;567:191-198. DOI: 10.1016/j.memsci.2018.09.028

[71] Wang H, Hao Y. Thermodynamic study of solar thermochemical methane steam reforming with alternating $\mathrm{H}_{2}$ and $\mathrm{CO}_{2}$ permeation membranes reactors. Energy Procedia. 2017;105: 1980-1985. DOI: 10.1016/j. egypro.2017.03.570 


\title{
Evaluation of PV-Wind Hybrid Energy System for a Small Island
}

\author{
Sajid Ali and Choon-Man Jang
}

\begin{abstract}
Hybrid renewable energy system (HRES) consists of more than one type of renewable energy technology such as wind and solar. The main application of such energy systems is to provide electricity to remote areas such as villages and islands, where no other means of power generation are available. Present study includes the basic information about the working methodology and other characteristics of HRES. Furthermore, two case studies of HRES have also been included to demonstrate the practical working of such energy systems. In first case study the performance of a small HRES, consisting of photovoltaic (PV) panels and wind turbines installed at Deokjeokdo island in South Korea, has been analyzed using real time measured experimental data. Second case study deals with the techno-economic optimization of HRES designed for fulfilling yearly electricity consumption of Deokjeokdo island. Out of multiple HRES solutions, two systems were declared as the optimal solutions based on lowest net present cost (NPC) and lowest levelized cost of energy (LCOE).
\end{abstract}

Keywords: renewable energy, hybrid energy system, economic feasibility, lowest net present cost

\section{Introduction}

In order to develop an eco-friendly island, it is necessary to make electricity, heat and water independence using renewable energy as an energy source. Present study is aimed at developing a sustainable eco-friendly energy and water-

independent community inside a small island called Deokjeokdo island-Incheon, South Korea. Considering the natural environment and geographical features with hills, a small communities called Urumsil town in Deokjeokdo island through ecofriendly energy sources based on a hybrid power system are developed. The hybrid power system consists of a small wind turbine, a photovoltaic panel, a pumped storage hydroelectricity and energy storage system. The renewable energy hybrid system can provide stable electricity and water to the island without greenhouse gas emission by fossil fuels. The Korea Institute of Civil Engineering and Building Technology (KICT) has signed a memorandum of understanding with Incheon city and is participating in the eco-island project centered on Deokjeokdo island. A local community in Deokjeokdo, Incheon city, was selected in 2013 to be developed as an environmentally-friendly energy and water independent community. A hybrid power system based on a small wind turbine, a photovoltaic panel, a pumped storage hydroelectricity and energy storage 
system was built. Through this arrangement, electricity is supplied to the community without diesel power generation.

\subsection{Hybrid renewable energy system (HRES)}

Hybrid renewable energy system (HRES) comprises of multiple sorts of sustainable power sources, for example, sun based and wind. The principle thought behind the idea of HRES is to give continues and maintainable supply of power to regions particularly far from primary terrains. A HRES can be associated with principle grid or it can likewise be an independent power producing unit, having its very own framework for storing surplus power, depending upon the nearby land conditions and some other monetary conditions. Figure 1 demonstrates the working structure of a conventional HRES with pumped hydro storage (PHS) as energy storage system (ESS).

Recently, many case studies of installing HRES at a remote location have been conducted around the globe. For instance, Perez-Navarro et al. [1] designed a hybrid system consisting of wind-biomass in order to compensate and stabilize the power production of a $40 \mathrm{MW}$ wind power plant in Spain. Apart from the main equipment, their designed HRES also consisted of other auxiliaries such as stand-by generators, separate ESS and biomass gasifier as well. The extra power generated by the biogas generator was used to compensate the low power production of wind farm. Borhanazad et al. [2] conducted a comprehensive study to investigate the wind conditions, solar radiations and hydro potential of multiple locations in Malaysia for rural electrification. Similarly, Zuberi et al. [3] estimated the biomass potential of Pakistan and concluded that biomass can contribute to generate almost $24 \%$ of the total electricity demand of the country. They covered biomasses such as municipal solid waste (MSW), bagasse and livestock in their study. They also presented an idea of stand-alone power generation system using biomass as raw fuel. Bhandari et al. [4] studied a very classic model of HRES for rural electrification, consisting of wind-PV-hydro as primary energy sources. They showed that installing such HRESs at very remote locations can be economically cheaper than connecting aforementioned areas with main grids. Mazzola et al. [5] designed a PVbiomass based HRES for a small town in India and conducted its economic feasibility as well. The authors mentioned that LCOE can be reduced up to $40 \%$ if

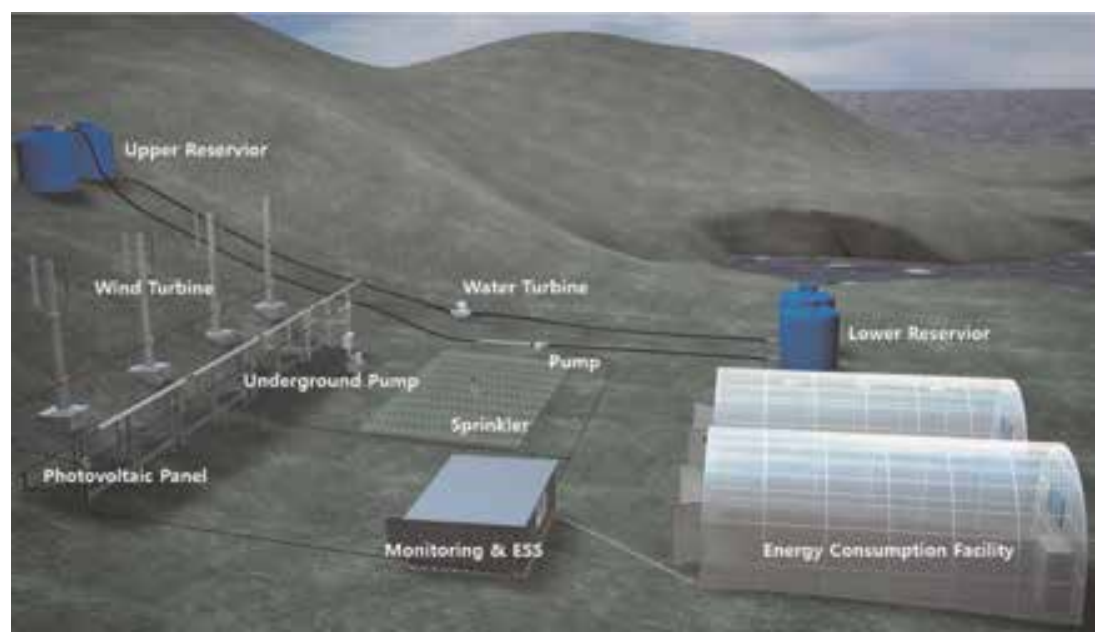

Figure 1.

Conceptual design of HRES. 
compared with electricity generation from diesel generators. Ahmad et al. [6] investigated the HRES consisting of wind-PV-biomass as primary energy sources for electrification of a small town in Pakistan called the Kallar Kahar. The study was conducted for multiple load conditions and authors recommended the installation of HRES near aforementioned site on the basis of strong economical conclusions.

\subsection{Deokjeokdo island in South Korea as test bed}

Deokjeokdo island (latitude: $37.22^{\circ}$, longitude: $126.15^{\circ}$ ) is the biggest island in the Ongjin-kun area in South Korea, arranged $50 \mathrm{~km}$ far from Incheon ocean port. At the end of 2013, the total population of Deokjeokdo island was approximately 5000 and its area is $21 \mathrm{~km}^{2}$. The island has a relatively large population engaged in

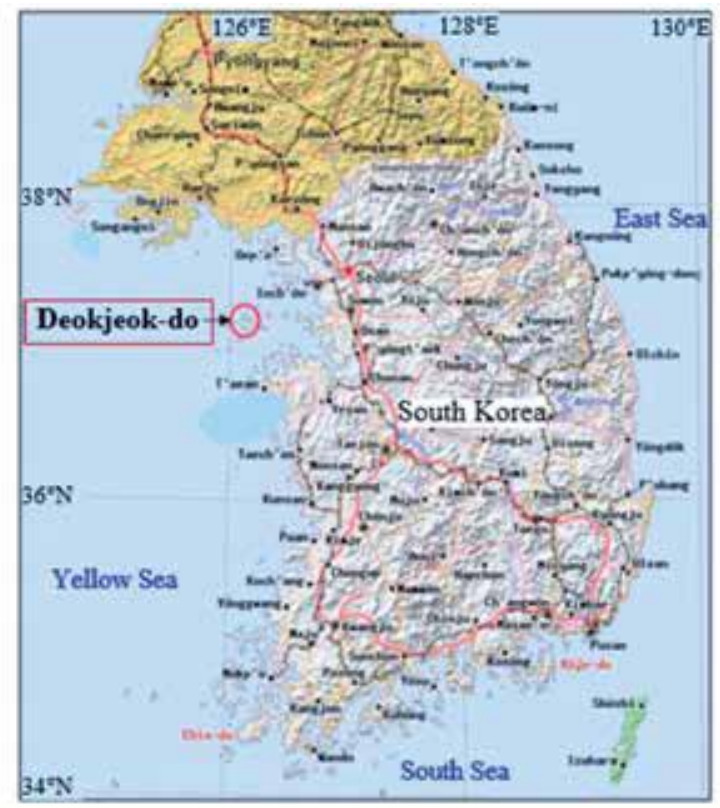

\section{(geographical location of the island)}

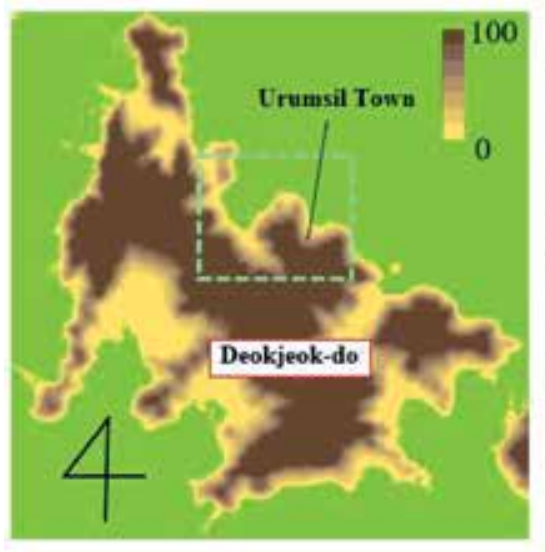

(top view of the island)

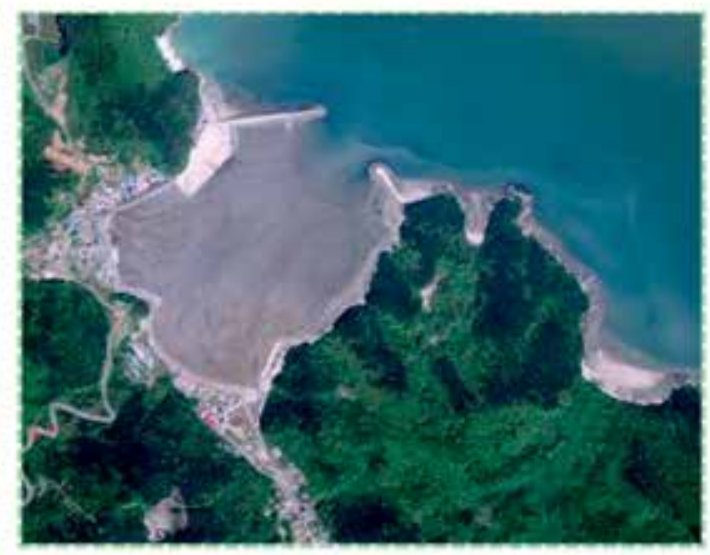

(test bed)

Figure 2.

Geographical location of Deokjeokdo island in South Korea. 
agriculture and tourism, rather than fishing, and is actively developing tourism resources as Green Island. This island is excessively long way from primary land of South Korea, so it is not monetarily suitable to associate it with main framework for power transmission. Subsequently, this island has its very own power generation system fueled by diesel. In any case, the local government has demonstrated its enthusiasm to make Deokjeokdo island, a green island as far as power generation is concerned. Present study investigates the sustainable power source potential at the mentioned site and after that recommends an ideal HRES dependent on economic assessments.

Figure 2 demonstrates geographical details of the Deokjeokdo including the Urumsil town and test bed of the hybrid renewable energy system.

\section{Analysis of experimental HRES installed at Deokjeokdo island}

Experimental HRES facility installed at Deokjeokdo island is shown in Figure 3. The experimental HRES consists of two Darrieus type vertical axis wind turbines (VAWT) and photovoltaic (PV) panels. The total capacity of this system is $24 \mathrm{~kW}$, with each wind turbine rated at $1.5 \mathrm{~kW}$ and solar panels of $3 \mathrm{~kW}$ capacity, respectively. In order to record the wind conditions such as wind speed and wind direction, a vertical tower called the "wind master" has been installed at the local site, as shown in Figure 3. Anemometer and anemoscope are attached on wind master to record wind speed and wind angle, respectively. Solar panels are inclined at $30^{\circ}$ to capture the maximum radiations from sun. This system was being monitored for two consecutive years, i.e., 2016 and 2018.

\subsection{Wind potential estimation}

Prior to assessing the power production from HRES, specifically from wind turbine, it is of immense importance to analyze the wind conditions of local site at first place. In current case, the wind data used for this purpose come from measured by wind master as mentioned above. Figure 4 shows the season wise plots of wind characteristics in the form of wind rose. It is clear from the figures that prevailing wind direction is south-west $\left(180-270^{\circ}\right)$; with most frequent wind speeds are in the range from 2 to $3 \mathrm{~m} / \mathrm{s}$ and spring is the "windiest" season. It is to be noted that wind data were measured at $10 \mathrm{~m}$ height.

Weibull probability density function (PDF) and cumulative density function (CDF) are two classical tools to study the wind characteristics of a region. Both functions can be defined as follows, respectively:

$$
\begin{gathered}
P D F=f(v)=(k / c)(v / c)^{k-1} \exp \left[-(v / c)^{k}\right](v>0 ; k, c>0) \\
C D F=F(v)=1-\exp \left[-(v / c)^{k}\right]
\end{gathered}
$$

where $k, c$ and $v$ are Weibull shape parameter, Weibull scale factor and wind speed, respectively. Shape factor and scale parameters are the defining parameters for Weibull distribution [7] and they determine the abscissa scale and the width of wind speed data distribution plot, respectively. There are many mathematical approaches to calculate $k$ and $c$ like graphical, maximum likelihood, empirical, power density and moment method [8]. Empirical method and method of Justus and Mikhail [9] will be used in this present study to estimate $k$ and $c$. 


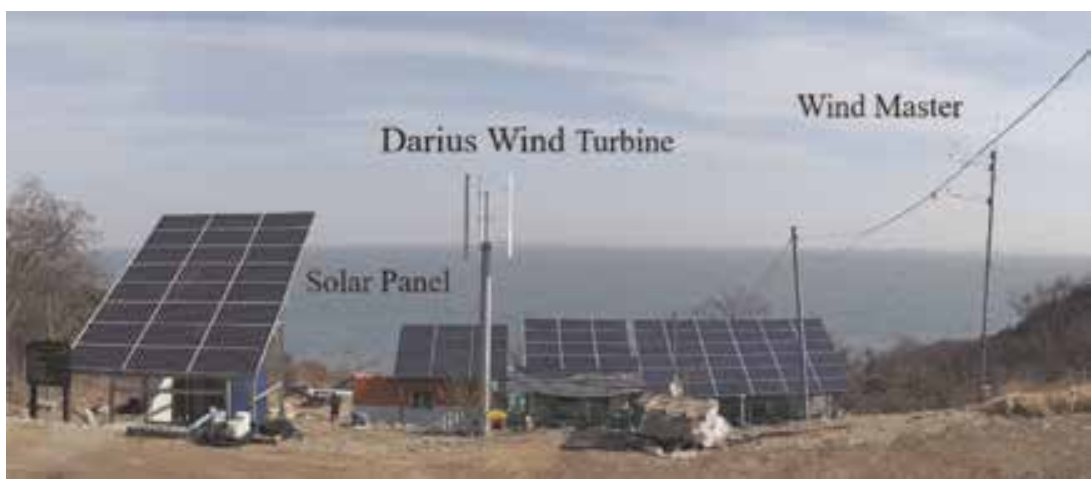

Figure 3.

Experimental HRES at Deokjeokdo island.

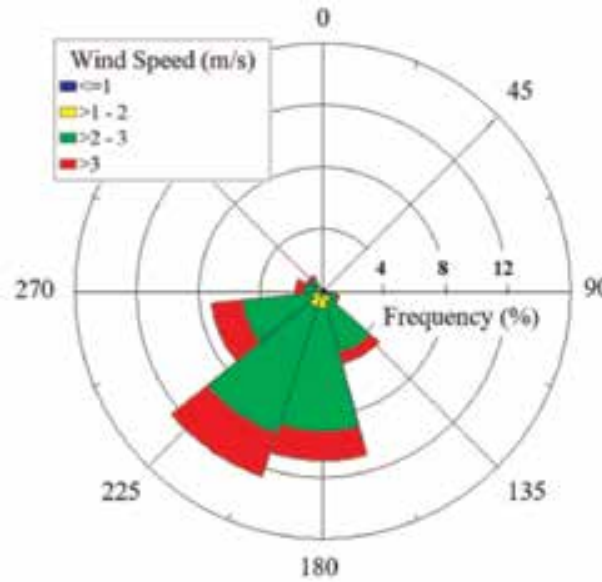

(a)

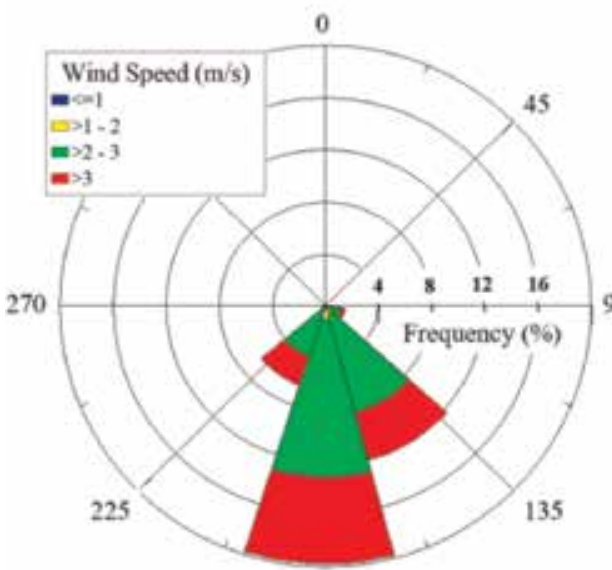

180

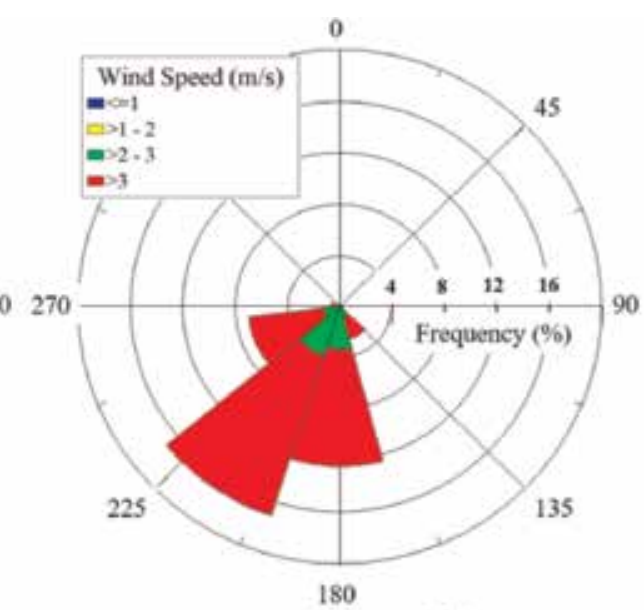

(b)

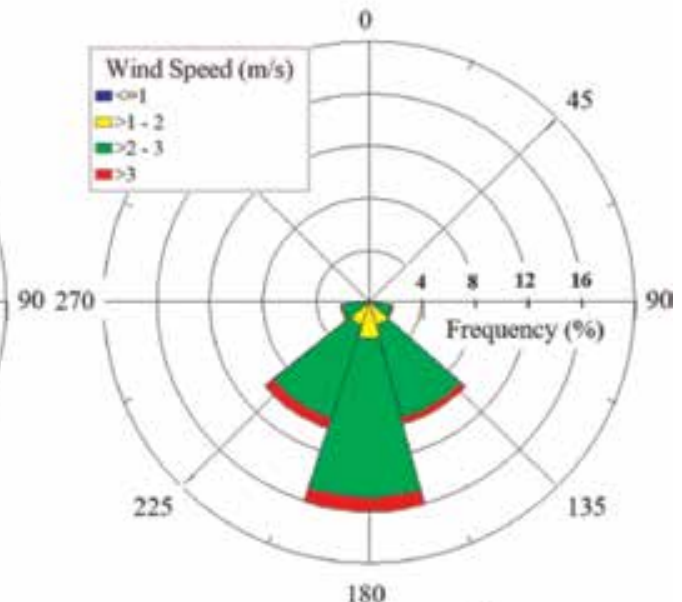

(d)

Figure 4 .

Wind rose at Deokjeokdo island (a) winter (b) spring (c) summer (d) fall. 


$$
\begin{gathered}
v_{m}=\frac{1}{n}\left[\sum_{i=1}^{n} v_{i}\right] \\
\sigma^{2}=\frac{1}{n-1} \sum_{i=1}^{n}\left(v_{i}-v_{m}\right)^{2}
\end{gathered}
$$

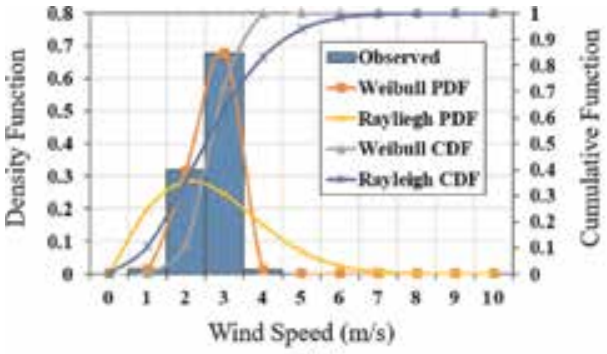

(a)

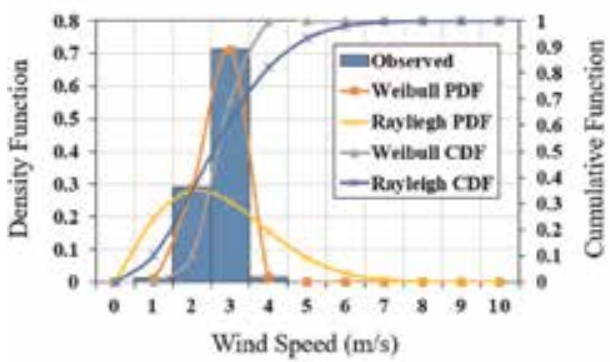

(c)

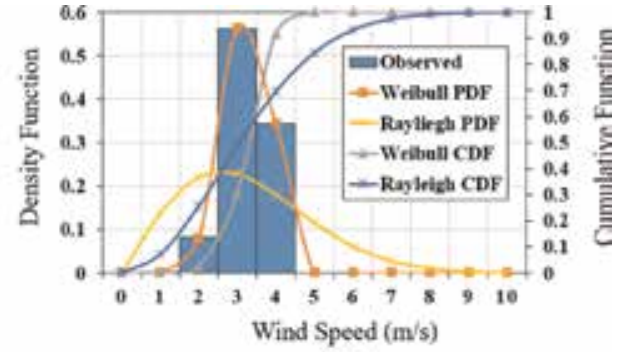

(b)

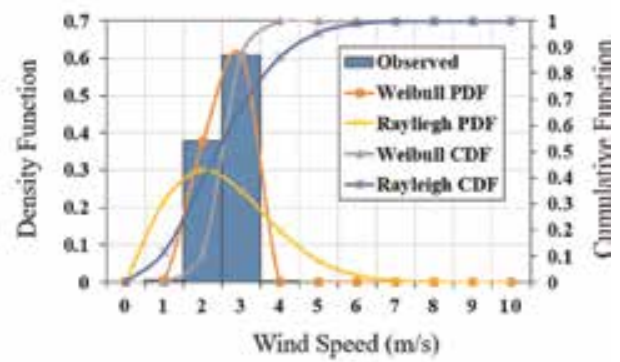

(d)

Figure 5.

\begin{tabular}{|c|c|c|c|c|c|c|c|c|c|c|c|c|}
\hline \multirow[t]{2}{*}{ Angle range $\left[{ }^{\circ}\right]$} & \multicolumn{12}{|c|}{ Percentage of total wind occurrence } \\
\hline & Jan & Feb & Mar & Apr & May & Jun & Jul & Aug & Sep & Oct & Nov & Dec \\
\hline$[0-30]$ & 0 & 0 & 0 & 0 & 0 & 0 & 0 & 0 & 0 & 0 & 0 & 0 \\
\hline$[30-60]$ & 0 & 0 & 0 & 0 & 0 & 0 & 0 & 0 & 0 & 0 & 0 & 0 \\
\hline$[60-90]$ & 0 & 0 & 0 & 0 & 0 & 0 & 0 & 0 & 0 & 0 & 0 & 0 \\
\hline$[90-120]$ & 2 & 0 & 0 & 0 & 0 & 1 & 1 & 2 & 2 & 1 & 1 & 1 \\
\hline [120-150] & 8 & 4 & 2 & 3 & 5 & 14 & 12 & 14 & 15 & 8 & 9 & 5 \\
\hline [150-180] & 23 & 18 & 13 & 23 & 29 & 43 & 43 & 36 & 36 & 24 & 28 & 17 \\
\hline [180-210] & 32 & 36 & 33 & 47 & 46 & 35 & 36 & 35 & 34 & 37 & 36 & 28 \\
\hline [210-240] & 26 & 31 & 35 & 24 & 19 & 6 & 7 & 12 & 12 & 24 & 20 & 26 \\
\hline [240-270] & 8 & 10 & 15 & 3 & 2 & 0 & 0 & 1 & 1 & 6 & 5 & 14 \\
\hline$[270-300]$ & 1 & 1 & 1 & 0 & 0 & 0 & 0 & 0 & 0 & 0 & 1 & 4 \\
\hline [300-330] & 0 & 0 & 0 & 0 & 0 & 0 & 0 & 0 & 0 & 0 & 0 & 4 \\
\hline [330-360] & 0 & 0 & 0 & 0 & 0 & 0 & 0 & 0 & 0 & 0 & 0 & 0 \\
\hline
\end{tabular}

Weibull plots at Deokjeokdo island (a) winter (b) spring (c) summer (d) fall.

Table 1.

Monthly variation in percentages of total wind speed according to wind direction ranges. 


$$
\begin{gathered}
k=\left(\frac{\sigma}{v_{m}}\right)^{-1.086}(1 \leq k \leq 10) \\
c=\frac{v_{m}}{\Gamma(1+1 / k)}
\end{gathered}
$$

Figure 5 shows the season wise Weibull plots for Deokjeokdo island prepared using 2 years measured data (2016 and 2017). These figures also show the curves for Rayleigh distributions (PDF and CDF), which are essentially Weibull distributions at $k=2$. Figure 5 reveals that the most frequently occurring wind during all the seasons is $3 \mathrm{~m} / \mathrm{s}$ and spring has high wind speeds, as it was also concluded above from Figure 4.

Table 1 explains the distribution of wind coming from different directions on monthly basis. Table 1 also concludes the same as Figure 4 that prevailing wind direction is south-west.

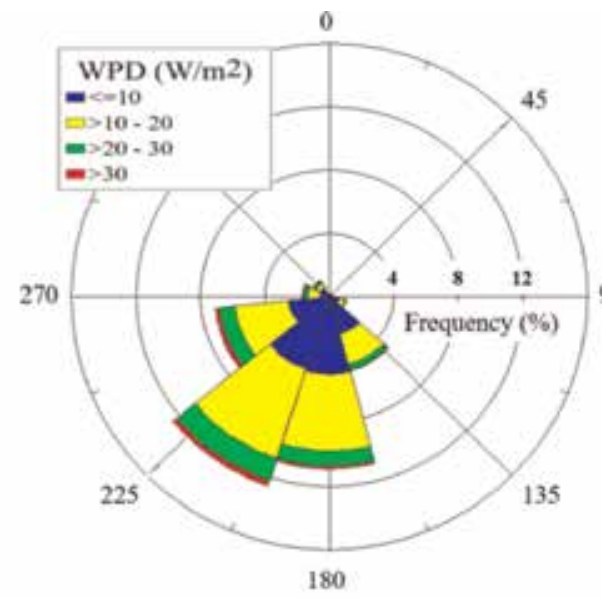

(a)

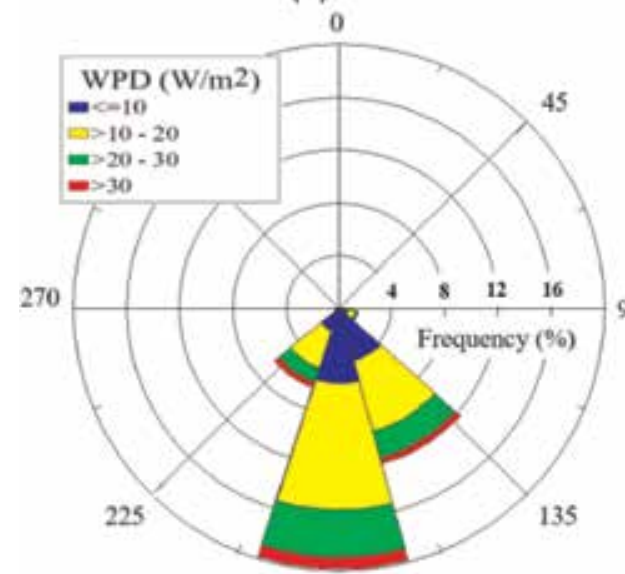

180

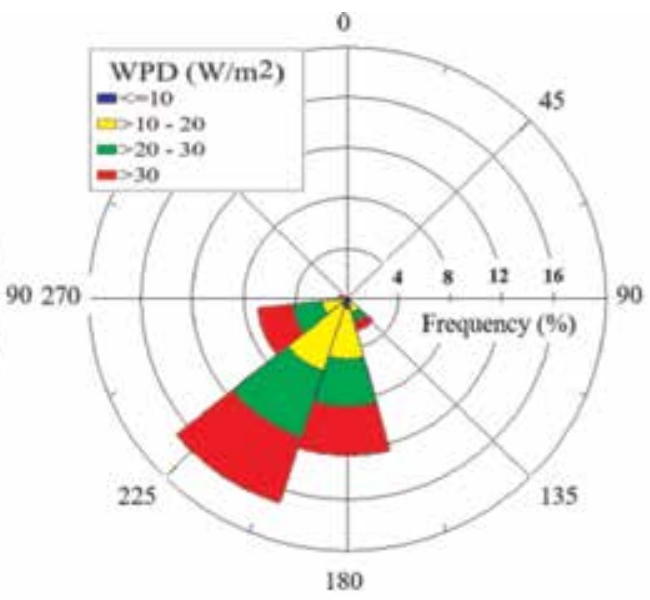

(b)

(c)

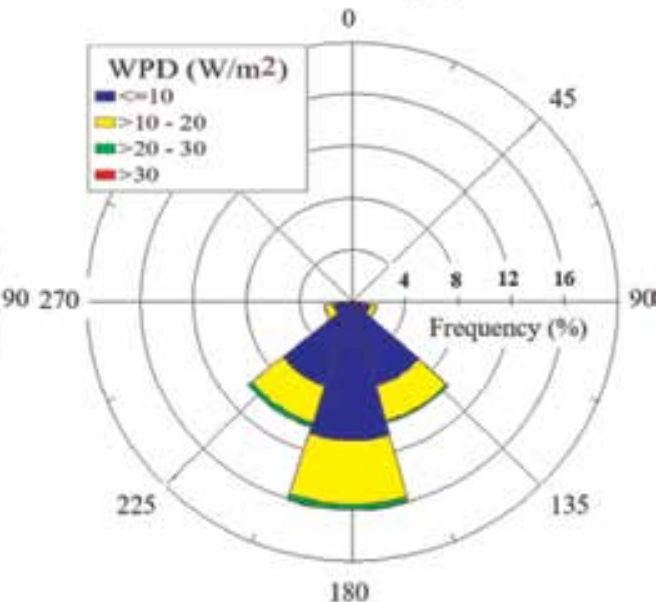

(d)

Figure 6.

Observed WPD at Deokjeokdo island (a) winter (b) spring (c) summer (d) fall. 
Figure 6 shows the wind power density (WPD) on the basis of seasons. The patterns being observed in Figure 6 are very much identical to patterns of Figure 4.

\subsection{Solar potential estimation}

Figure 7 shows the average solar radiations $\left(\mathrm{W} / \mathrm{m}^{2}\right)$ over different major cities of South Korea. Daejeon has the highest solar radiations value $\left(175 \mathrm{~W} / \mathrm{m}^{2}\right)$ whereas Seoul has the lowest $\left(145 \mathrm{~W} / \mathrm{m}^{2}\right)$.

Similarly, Figure 8 shows the average values of daily solar radiations and clearness index over Deokjeokdo island, on monthly basis.

\subsection{Estimation of power production from wind turbine}

This section presents the results such as power production from small Darrieus VAWT. Table 2 summarizes the important details about the wind turbine whereas Figure 9(a) shows the geometrical dimensions and Figure 9(b) shows the power curve of wind turbine installed at Deokjeokdo island. The blade height and chord of the turbine rotor are 3 and $0.2 \mathrm{~m}$, respectively. Design blade section profile is NACA0015, while the rotational diameter of the turbine rotor is $2 \mathrm{~m}$. Rated wind speed and rotor rotational speed are $13.5 \mathrm{~m} / \mathrm{s}$ and $300 \mathrm{rpm}$, respectively.

Figure 10 shows diagram for data acquisition system to obtain experimental data from the wind turbine and the wind master. Turbine performance data is measured between turbine and power transducer, thus contains power generator loss. Power output is stored in battery bank first, then supplied to users after converting to AC voltages.

The commercial code, SC/Tetra, has been employed in the present numerical simulation. It solves the governing fluid dynamics equations, which consist of

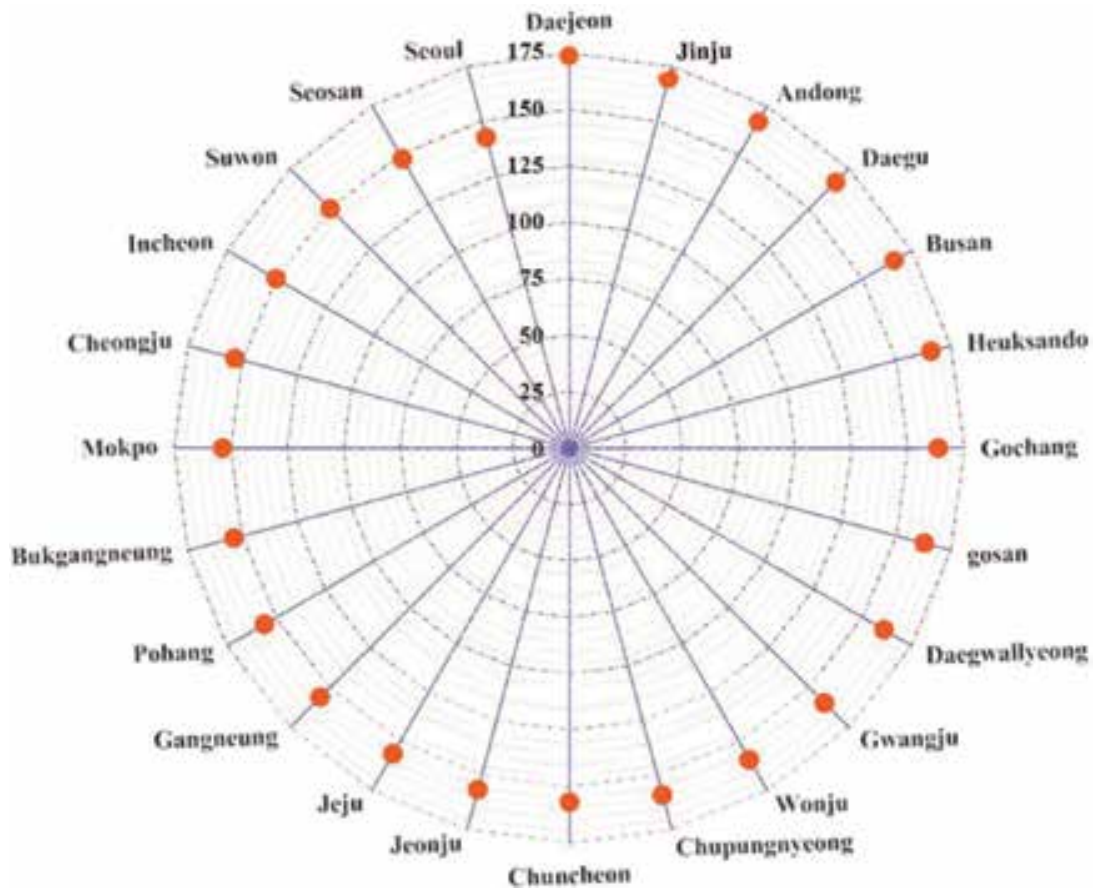

Figure 7.

Solar radiations over different cities of South Korea $\left(W / m^{2}\right)[10]$. 
Evaluation of PV-Wind Hybrid Energy System for a Small Island

DOI: http://dx.doi.org/10.5772/intechopen.85221

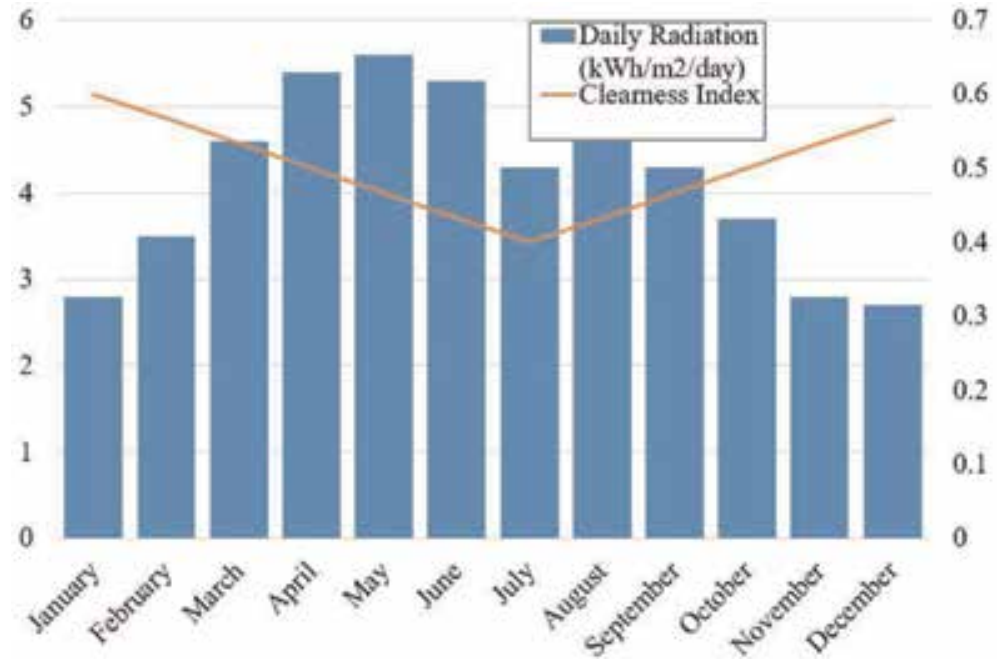

Figure 8.

Solar radiations over Deokjeokdo island [10].

\begin{tabular}{lc}
\hline Parameter & Value \\
\hline Rated power, $\mathrm{kW}$ & 1.5 \\
\hline Rated wind speed, $\mathrm{m} / \mathrm{s}$ & 13.5 \\
\hline Rated rotational speed, RPM & 300 \\
\hline Cut-in wind speed, $\mathrm{m} / \mathrm{s}$ & 3 \\
\hline Chord length, $\mathrm{m}$ & 0.2 \\
\hline Blade length (height), $\mathrm{m}$ & 3 \\
\hline Rotational diameter, $\mathrm{m}$ & 2 \\
\hline Blade profile & NACA0015 \\
\hline
\end{tabular}

Table 2.

Specifications of test Darrieus wind turbine.

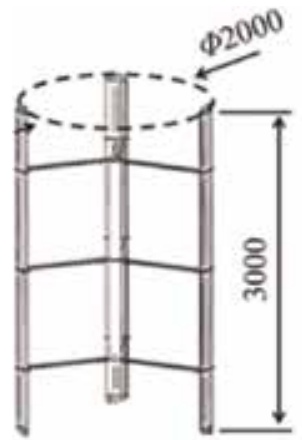

(a)

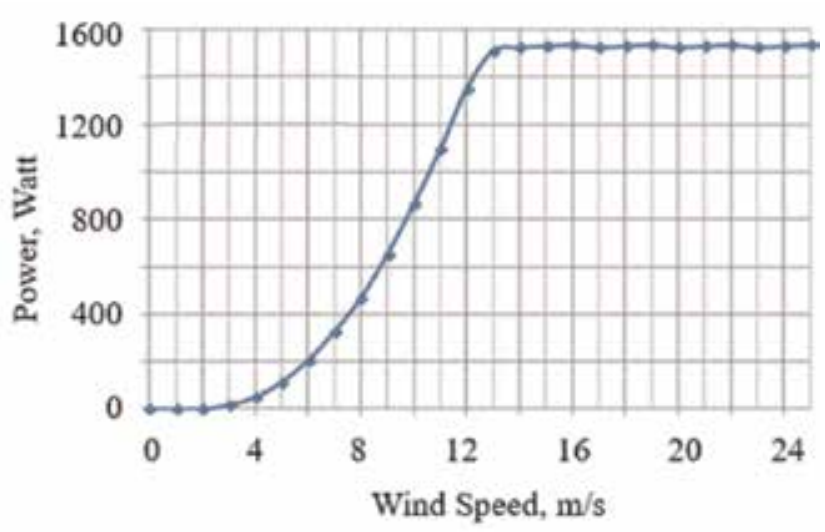

(b)

Figure 9.

Darrieus wind turbine installed at Deokjeokdo island (a) rotor dimensions (b) power curve. 
continuity and unsteady Reynolds averaged Navier-Stokes (URANS) equations. The computational domain which consists of rotational and stationary domains, is shown in Figure 11. Tetrahedral, prism and pyramid elements have been used overall but mostly only tetrahedral element type is employed. The total number of meshing elements is around 13 million, whereas the total number of nodes is approximately 3.5 million in complete domain. Shear stress transport (SST) model with a scalable wall function is employed to estimate eddy viscosity. In terms of the boundary conditions, a velocity of $5 \mathrm{~m} / \mathrm{s}$ is specified at the inlet, and natural outflow condition is imposed at the outlet.

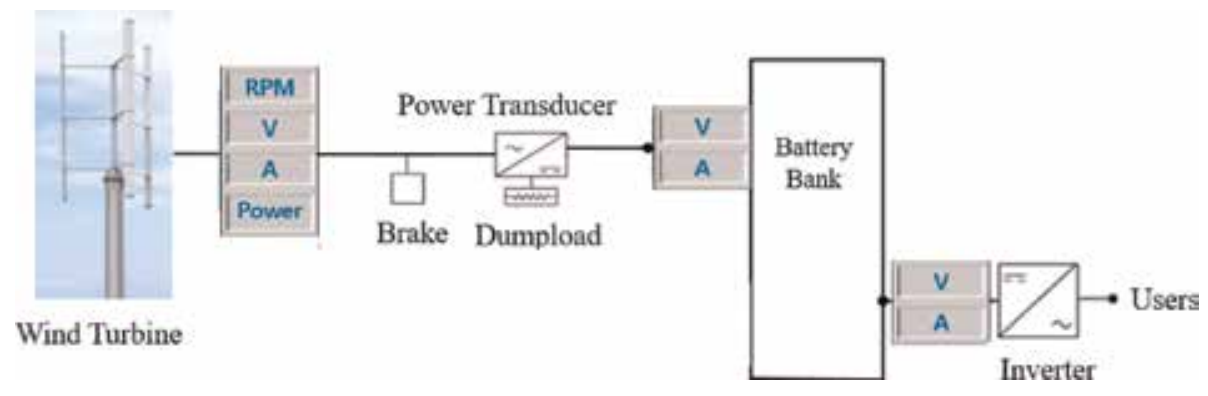

Figure 10.

Diagram for data acquisition system.
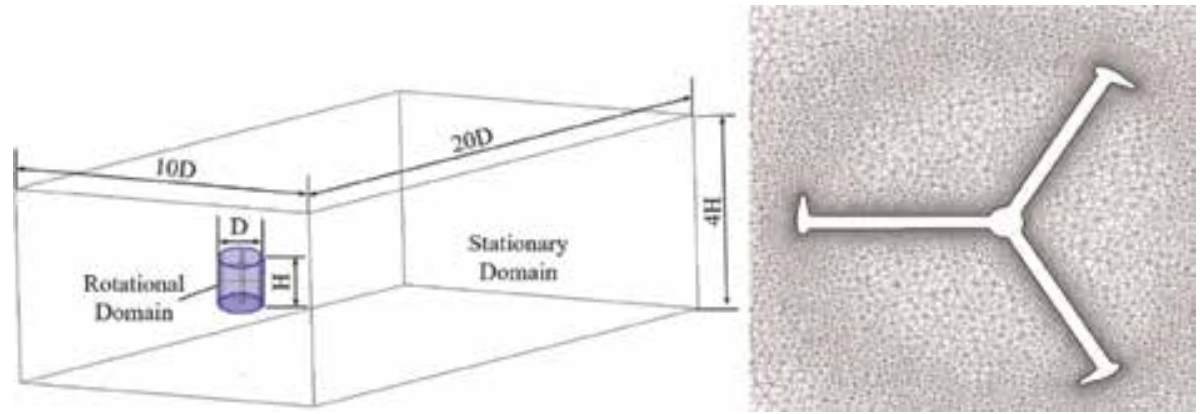

Figure 11.

Computational domain (left) and grid system around turbine rotor (right).
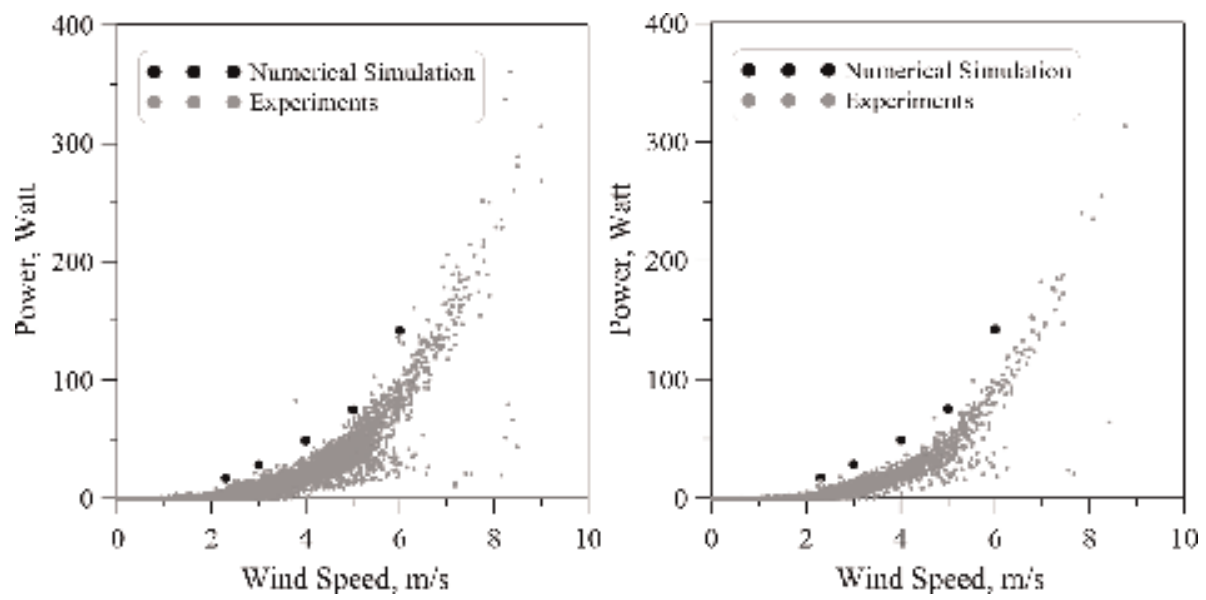

Figure 12.

Comparisons of turbine power between numerical simulation and experimental measurement for 10-min average (left) and 3o-min average (right). 


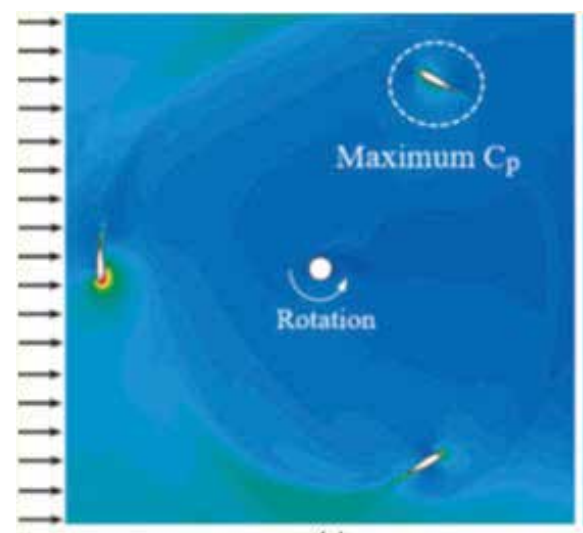

(a)

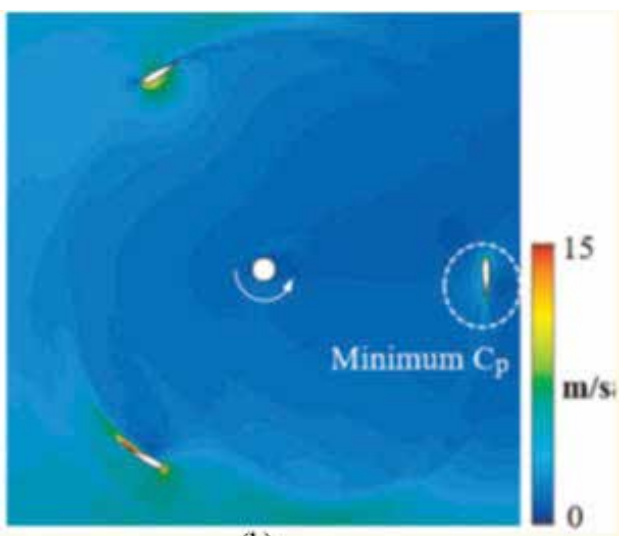

(b)

Figure 13.

Wind speed around turbine rotor with wind speed of $5 \mathrm{~m} / \mathrm{s}$ (a) Rotor orientation for maximum $C_{P}$ (b) Rotor orientation for minimum $C_{P}$.

Figure 12 shows the comparisons of turbine power between numerical simulation and experimental measurement for two time averages. In the figure, turbine power obtained by numerical simulation has similar trend to the experimental result. Especially turbine power determined by 10 -min average is more similar to the results of numerical simulation compared to 30 -min average. This is considered that 10-min average step having lower SD is more effective to analyze the performance of a small vertical wind turbine. From the above comparisons, it can be said that turbine power obtained by numerical simulation is correctly analyzed.

Figure 13 shows contours of wind speed around turbine rotor at two different rotation positions, where maximum and minimum values of power coefficient $\left(\mathrm{C}_{\mathrm{p}}\right)$ occurred, during one complete revolution. Blade having maximum power surrounded by dashed line in the left side is located at the blade rotation angle of $240^{\circ}$ where maximum wind velocity around the blade is occurred without large separation flow along the blade surface. An increase in linear speed of the blade leads to increase the rotational speed of rotor and eventually overall power output is enhanced. Larger separated flow is observed at the blade having minimum power because inflow in front of turbine rotor directly interfaces to the blade surface.

\section{Optimal design of HRES}

This section describes an optimum HRES for Deokjeokdo island based on lowest net present cost (NPC) and levelized cost of energy (LCOE) using HOMER pro software model. Hybrid optimization model for electric renewables (HOMER) pro software can efficiently model and optimize renewable energy plans for a specific region. The optimum HRES must fulfill hourly and annual electricity demand of the island, which corresponds to approximately 7.296 MWh/year without any external assistance such as grid, etc.

\subsection{Input data for HOMER pro}

Before starting energy simulations in HOMER pro, one must define pre requisites such as electric load, equipment such as wind turbines, PV panels and other essential details like interest rate and project life. 


\subsubsection{Electric load}

In order to optimally design a HRES, electric load information such as peak load, daily average electricity consumption and hourly load profile are of critical importance. The maximum availability of load information enables designing a more compact HRES. In the present case, the load data were not collected from any official government source (because of unavailability of such data), but from a previous study on Deokjeokdo island [11]. The average daily load was found to be approximately $24,720 \mathrm{kWh}$ with peak load of $2292 \mathrm{~kW}$ typically occurring during winter season and total annual electricity consumption corresponds to a value of $7.296 \mathrm{GWh}$. The electricity consumption during winter season is higher than rest of the seasons due to the extensive use of space heating equipment powered by electricity. Figure 14 shows the daily and monthly electricity consumption at Deokjeokdo island.

\subsubsection{Equipment selection}

Table 3 presents all technical and economic details about the selected equipment for the study. It is to be noted that all the equipment have been selected by default by HOMER pro except wind turbine; which has been selected after a detailed analysis of wind characteristics at Deokjeokdo island by same authors in Ali et al. [12].

\subsubsection{HOMER pro model}

Figure 15 shows the HOMER pro model built for current study with two electric transmission lines, i.e., DC and AC. Basically, the electricity generated from each energy source is stored in the battery based on the DC line. This is because, in comparison with AC, small-scale power generation systems can reduce losses due to electricity conversion. Electric load is used after converting it to AC by using the converter as shown in the figure.

Table 4 displays the values of all the sensitivity variables considered in current study. First values of all sensitivity variables in Table 4, makes the default case.

\subsection{Optimum HRES solutions}

This section presents the characteristics and analysis of the most optimal HRESs recommended for Deokjeokdo island on the basis of techno-economic evaluations.

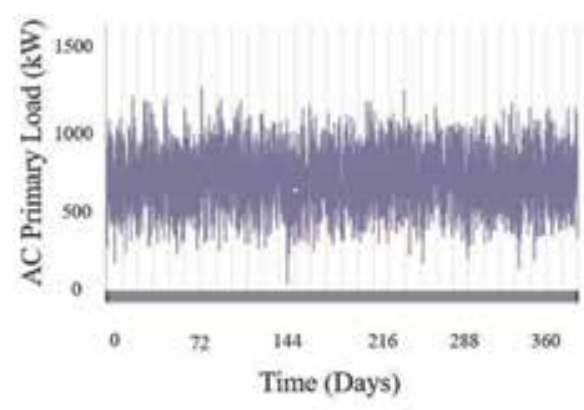

(a)

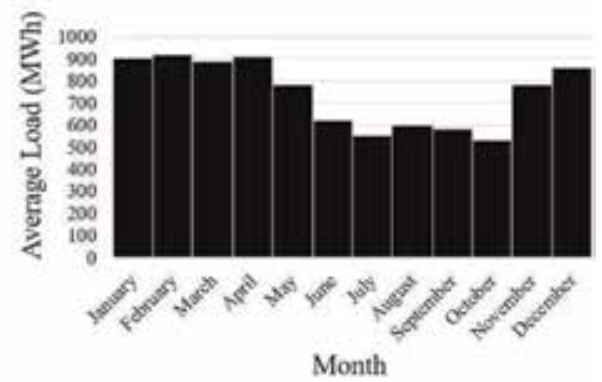

(b)

Figure 14.

Total AC electric load at Deokjeok island (a) daily load (b) monthly load. 
Evaluation of PV-Wind Hybrid Energy System for a Small Island

DOI: http://dx.doi.org/10.5772/intechopen.85221

(a) Converter

\begin{tabular}{lccccccc}
\hline Model & $\begin{array}{c}\text { Capital } \\
(\$ / \mathbf{k W})\end{array}$ & $\begin{array}{c}\text { Replacement } \\
(\$ / \mathbf{k W})\end{array}$ & $\begin{array}{c}\text { O\&M } \\
(\$ / \text { year })\end{array}$ & $\begin{array}{c}\text { Lifetime } \\
(\text { years })\end{array}$ & $\begin{array}{c}\text { Inverter } \\
\text { efficiency } \\
(\%)\end{array}$ & $\begin{array}{c}\text { Rectifier } \\
\text { capacity } \\
(\%)\end{array}$ & $\begin{array}{c}\text { Rectifier } \\
\text { efficiency } \\
(\%)\end{array}$ \\
\hline $\begin{array}{l}\text { Leonics } \\
\text { MTP-413F } \\
\text { 25kW }\end{array}$ & 800 & 800 & 10 & 10 & 96 & 80 & 94 \\
\hline
\end{tabular}

(b) Battery

\begin{tabular}{lccccccc}
\hline Model & $\begin{array}{c}\text { Capital } \\
(\$ / \text { battery })\end{array}$ & $\begin{array}{c}\text { Replacement } \\
(\$ / \text { battery })\end{array}$ & $\begin{array}{c}\text { O\&M } \\
(\$ / \text { year })\end{array}$ & $\begin{array}{c}\text { Lifetime } \\
(\text { years })\end{array}$ & $\begin{array}{c}\text { Initial state } \\
\text { of charge } \\
(\%)\end{array}$ & $\begin{array}{c}\text { Nominal } \\
\text { voltage } \\
(\mathbf{V})\end{array}$ & $\begin{array}{c}\text { Nominal } \\
\text { capacity } \\
(\mathbf{k W h})\end{array}$ \\
\hline $\begin{array}{l}\text { Surrette } \\
\text { 6CS25P } \\
\text { (kinetic) }\end{array}$ & 250 & 250 & 1 & 20 & 100 & 6 & 6.91 \\
\hline
\end{tabular}

(c) PV panel

\begin{tabular}{cccccccc}
\hline Model & $\begin{array}{c}\text { Capital } \\
(\mathbf{\$} / \mathbf{k W})\end{array}$ & $\begin{array}{c}\text { Replacement } \\
(\mathbf{\$} / \mathbf{k W})\end{array}$ & $\begin{array}{c}\text { O\&M } \\
(\mathbf{\$} / \text { year })\end{array}$ & $\begin{array}{c}\text { Lifetime } \\
(\text { years })\end{array}$ & $\begin{array}{c}\text { Derating } \\
\text { factor }(\%)\end{array}$ & $\begin{array}{c}\text { Rated } \\
\text { capacity } \\
(\mathbf{k W})\end{array}$ & $\begin{array}{c}\text { Efficiency } \\
(\%)\end{array}$ \\
\hline $\begin{array}{l}\text { CS6X- } \\
\text { 325P }\end{array}$ & 1500 & 1500 & 30 & 25 & 88 & 50 & 17 \\
\hline
\end{tabular}

(d) Wind turbine [10]

\begin{tabular}{lcccccccc}
\hline Model & $\begin{array}{c}\text { Capital (\$/ } \\
\text { wind } \\
\text { turbine) }\end{array}$ & $\begin{array}{c}\text { Replacement } \\
\text { (\$/wind } \\
\text { turbine) }\end{array}$ & $\begin{array}{c}\text { O\&M } \\
(\$ / \text { year) }\end{array}$ & $\begin{array}{c}\text { Lifetime } \\
\text { (years) }\end{array}$ & $\begin{array}{c}\text { Hub } \\
\text { height } \\
(\mathbf{m})\end{array}$ & $\begin{array}{c}\text { Wake } \\
\text { loss } \\
(\%)\end{array}$ & $\begin{array}{c}\text { Other } \\
\text { losses } \\
(\%)\end{array}$ & $\begin{array}{c}\text { Rated } \\
\text { capacity } \\
(\mathbf{k W})\end{array}$ \\
\hline $\begin{array}{l}\text { STX } \\
93 / 2000\end{array}$ & $2,869,747$ & $2,869,747$ & 110,375 & 25 & 80 & 5 & 10 & 1500 \\
\hline
\end{tabular}

Table 3.

Selected equipment.

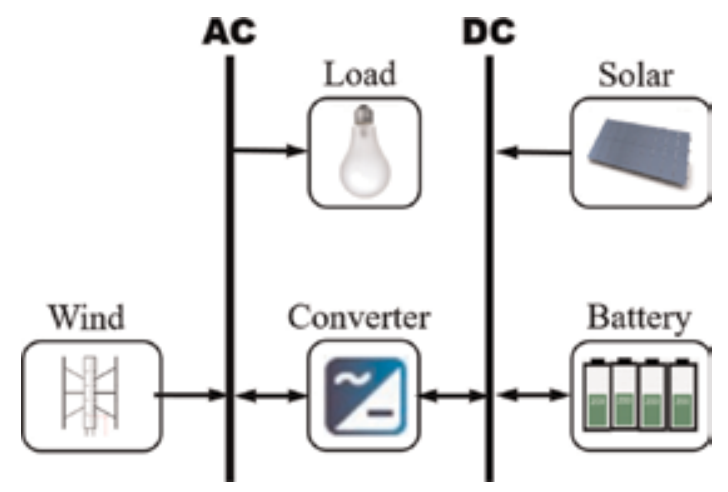

Figure 15.

HOMER pro model constructed for current study.

HOMER simulations generated a total of 551,035 alternatives, out of which only 232,683 solutions were found to be feasible. Figure 16 shows the breakdown of all the solutions generated and it also specifies the multiple reasons for omitted solutions.

Out of the 232,683 feasible solutions, only following two HRESs were finalized as the most suitable options. 
- System A: HRES with lowest overall net present cost (NPC)

- System B: HRES with lowest overall levelized cost of energy (LCOE)

Table 5 shows the basic characteristics of both of the optimized system solutions. It is to be noted that both systems have batteries as default option for storing surplus electricity. System A has the lowest overall NPC (11.3 million \$) whereas LCOE is lowest in case of system B (\$0.123). Table 5 also displays the values of

\begin{tabular}{lcc}
\hline Average load (kWh/day) & Discount rate $(\%)$ & Project lifetime (years) \\
\hline 24,720 & 8 & 25 \\
\hline 20,000 & 6 & 20 \\
\hline 30,000 & 4 & 15 \\
\hline
\end{tabular}

Table 4 .

Sensitivity variables.

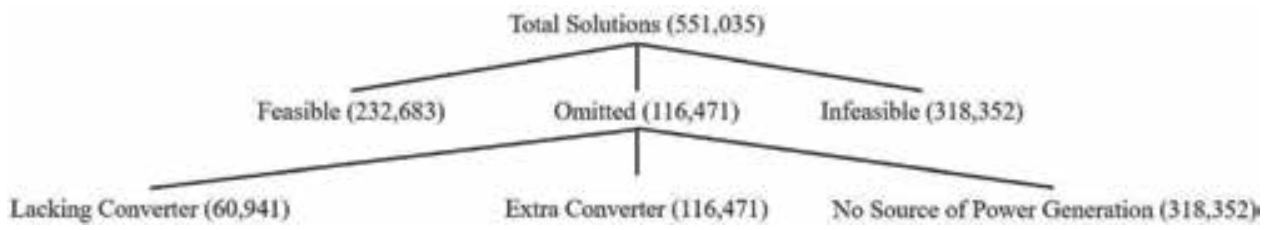

Figure 16.

Breakdown of multiple solutions obtained from HOMER simulations.

\begin{tabular}{lccc}
\hline Variable & Unit & System A & System B \\
\hline NPC & Million $\$$ & 11.3 & 17.61 \\
\hline LCOE & $\$ / \mathrm{kWh}$ & 0.158 & 0.123 \\
\hline Total load scaled average & $\mathrm{kWh} /$ day & 20,000 & 20,000 \\
\hline Nominal discount rate & $\%$ & 8 & 4 \\
\hline Project lifetime & years & 15 & 25 \\
\hline
\end{tabular}

Table 5 .

Basic information about both system solutions.

\begin{tabular}{lcccc}
\hline Component & Model & Unit & Size (system A) & Size (system B) \\
\hline PV panels & $\begin{array}{c}\text { Canadian Solar Max Power CS6X- } \\
\text { 325P }\end{array}$ & $\mathrm{kW}$ & 2504 & 3157 \\
\hline Battery & Surrette 6 CS 25P & Strings & 7197 & 6269 \\
\hline Wind turbine & STX 93/2000 & ea. & 1 & 1 \\
\hline $\begin{array}{l}\text { System } \\
\text { converter }\end{array}$ & Leonics MTP-413F 25 kW & $\mathrm{kW}$ & 1006 & 1009 \\
\hline $\begin{array}{l}\text { Dispatch } \\
\text { strategy }\end{array}$ & HOMER cycle charging & N/A & N/A & N/A \\
\hline
\end{tabular}

Table 6.

Systems architecture. 
sensitivity variables at which optimal system solutions have been obtained. Project life of system A (15 years) is less than that of system B ( 25 years), which is also one of the reasons for low NPC of system A.

Table 6 displays the selected size of each component for both systems. Both systems consist of one wind turbine and system converter of almost $1000 \mathrm{~kW}$ size. PV panel size for system B $(3,157 \mathrm{~kW})$ is higher than system A $(2,504 \mathrm{~kW})$, that is why NPC of system B is higher than system A. By selecting an appropriate model of wind turbine according to the wind conditions of Deokjeokdo island, both system architectures might be different from present cases. But, a right choice of wind

\begin{tabular}{lccc}
\hline Pollutant & Unit & Quantity (system A) & Quantity (system B) \\
\hline Carbon dioxide & $\mathrm{kg} /$ year & 795 & 700 \\
\hline Carbon monoxide & $\mathrm{kg} /$ year & 8.83 & 7.77 \\
\hline Unburned hydrocarbons & $\mathrm{kg} /$ year & 0 & 0 \\
\hline Particulate matter & $\mathrm{kg} /$ year & 0 & 0 \\
\hline Sulfur dioxide & $\mathrm{kg} /$ year & 0 & 0 \\
\hline Nitrogen oxides & $\mathrm{kg} /$ year & 5.52 & 4.86 \\
\hline
\end{tabular}

Table 7.

Pollutants emission.

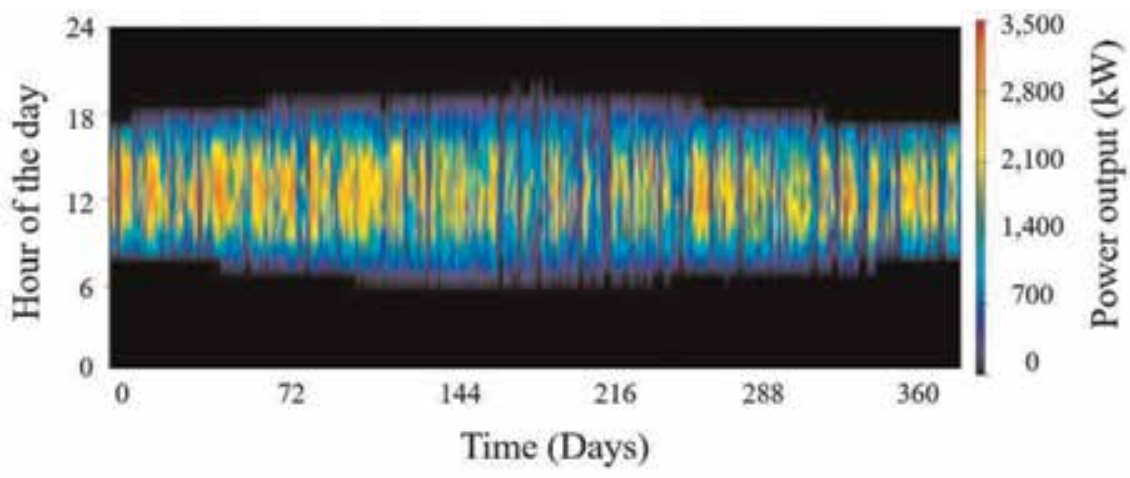

(a)

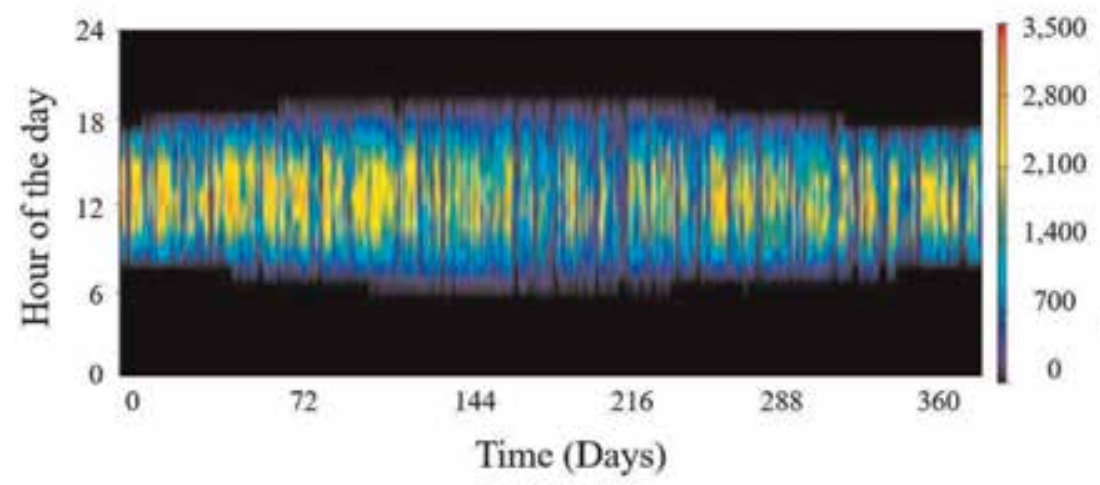

Figure 17.

Daily PV power output for both systems (a) system $A(b)$ system $B$. 
turbine depends on the detailed wind data analysis of local site, which is beyond the scope of current study; therefore not performed here.

Finally, Table 7 shows the annual amount of pollutant gases emissions due to operation of both systems.

Figure 17 displays the graphical representation of power produced by PV panels in both cases. Both the images of Figure 17 indicate that summer is the ideal season for harvesting energy from sun in South Korea, as the average day-time is almost 14-15 hours in Deokjeokdo island. The average hourly power generated by PV panels in case of system $\mathrm{A}$ is $425 \mathrm{kWh}$ whereas this value corresponds to $536 \mathrm{kWh}$ for system B.

\subsection{Sensitivity cases}

Although the most optimal system solutions have already been explained in detail in above sections. But it is also of critical importance to briefly explain some

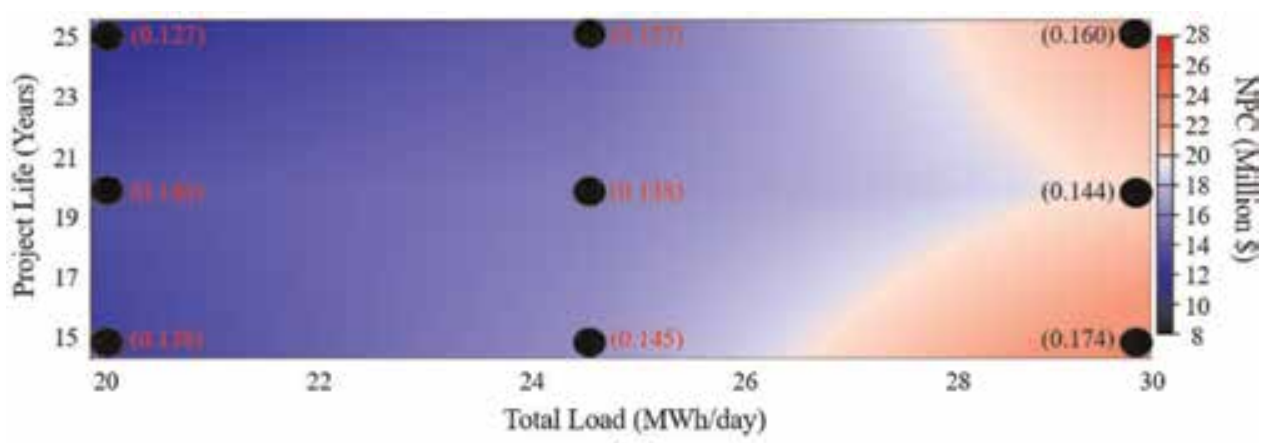

(a)

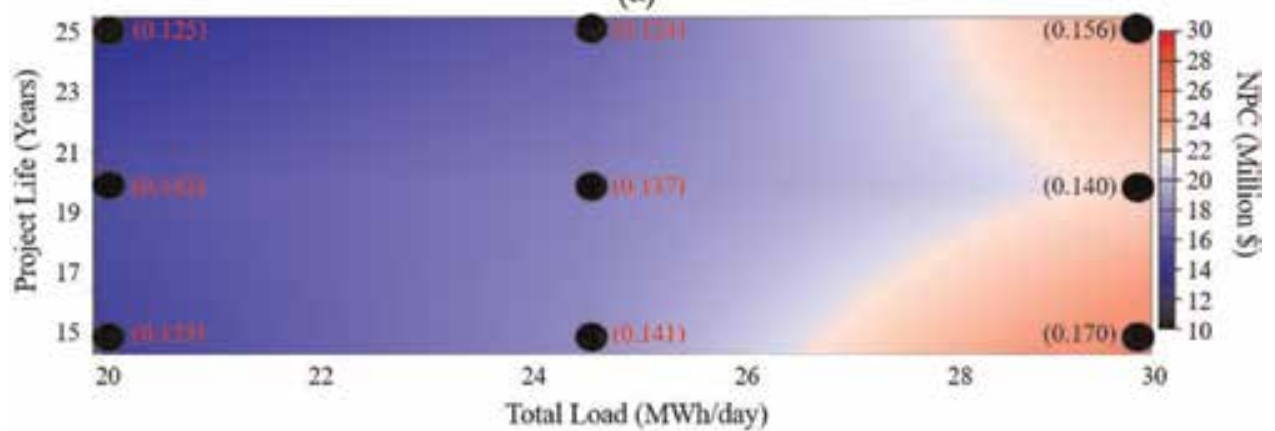

(b)

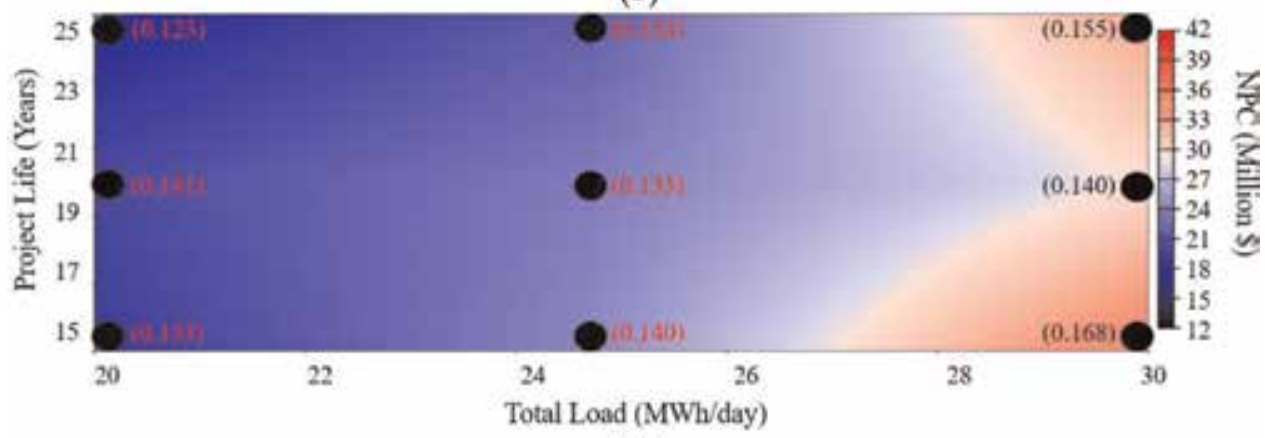

(c)

Figure 18.

Multiple system solutions with LCOE superimposed over NPC (a) discount rate $=4 \%(b)$ discount rate $=6 \%$ (c) discount rate $=8 \%$. 
of the other alternate system solutions on the basis of economic evaluations. In order to achieve this goal, Figure 18 has been prepared which shows multiple system solutions obtained by superimposing NPC over LCOE.

Figure 18 shows a total of 27 optimal system solutions obtained by varying the values of all sensitivity variables mentioned in Table 4 .

\section{Conclusions}

The present study provides a basic information about the working methodologies of a hybrid renewable energy system (HRES) consisting of wind and solar as primary energy resources. Two case studies of HRES have also been included to further clarify the economic aspects of such energy systems.

First case study deals with the analysis of a small HRES consisting of wind turbines and PV panels with batteries as energy storage system (ESS). This small HRES is being installed at Deokjeokdo island in South Korea and its performance have been monitored for two consecutive years (2016 and 2017). Analysis showed that the prevailing wind direction at Deokjeokdo island is either north-east or south-west, with mean wind speed of $3.6 \mathrm{~m} / \mathrm{s}$ at $10 \mathrm{~m}$ height. Similarly, average value of daily solar radiations was estimated to be $4.13 \mathrm{kWh} / \mathrm{m}^{2}$ with mean clearness index of 0.5 . The total capacity of this small HRES is $6 \mathrm{~kW}$; with two Darrieus VAWTs of $1.5 \mathrm{~kW}$ size each and $3 \mathrm{~kW}$ size of PV panels.

Second case study finds an optimum HRES to fulfill the yearly electricity demand of Deokjeokdo island, which corresponds to approximately 7.296 MWh/ year. Over 8760 simulations were performed to find out two optimum HRESs based on lowest NPC (system A) and lowest LCOE (system B), respectively. The overall NPC of system A was calculated to be 11.29 million USD, whereas for system B, it was 17.61 million USD. On the other hand, LCOE for system A was slightly higher than system B as it was $0.158 \$ / \mathrm{kWh}$ for system A and $0.123 \$ / \mathrm{kWh}$ for system $\mathrm{B}$. Both systems can independently provide electricity to Deokjeokdo island throughout the year without any external assistance such as grid, etc.

\section{Acknowledgements}

This study was supported with major project funding from the Korea Institute of Civil Engineering and Building Technology. We would also like to thank the KMA for providing long-term measured wind data at all proposed wind farm sites.

\section{Conflict of interest}

The authors declare no actual or potential conflicts of interest. 


\section{Author details}

Sajid $\mathrm{Ali}^{1,2}$ and Choon-Man Jang ${ }^{1,2 *}$

1 Smart City Construction Engineering, University of Science and Technology (UST), Daejeon, Korea

2 Department of Land, Water and Environmental Research, Korea Institute of Civil Engineering and Building Technology, Goyang-si, Korea

*Address all correspondence to: jangcm@kict.re.kr

\section{IntechOpen}

(C) 2019 The Author(s). Licensee IntechOpen. This chapter is distributed under the terms of the Creative Commons Attribution License (http://creativecommons.org/licenses/ by/3.0), which permits unrestricted use, distribution, and reproduction in any medium, provided the original work is properly cited. (c) BY 


\section{References}

[1] Perez-Navarro A, Alfonso D, Alvarez C, Ibanez F, Sanchez C, Segura I. Hybrid biomass-wind power plant for reliable energy generation. Renewable Energy. 2010;35:1436-1443

[2] Borhanazad H, Mekhilef S, Saidur R, Boroumandjazi G. Potential application of renewable energy for rural electrification in Malaysia. Renewable Energy. 2013;59:210-219

[3] Zuberi MJS, Torkmahalleh MA, Ali $\mathrm{SMH}$. A comparative study of biomass resources utilization for power generation and transportation in Pakistan. International Journal of Hydrogen Energy. 2015;40:11154-11160

[4] Bhandari B, Lee KT, Lee CS, Song CK, Maskey RK, Ahn SH. A novel offgrid hybrid power system comprised of solar photovoltaic, wind, and hydro energy sources. Applied Energy. 2014; 133:236-242

[5] Mazzola S, Astolfi M, Macchi E. The potential role of solid biomass for rural electrification: A techno economic analysis for a hybrid micro grid in India. Applied Energy. 2016;169:370-383

[6] Ahmad J, Imran M, Khalid A, Iqbal W, Ashraf SR, Adnan M, et al. Techno economic analysis of a windphotovoltaic-biomass hybrid renewable energy system for rural electrification: A case study of Kallar Kahar. Energy.

2018;148:208-234

[7] Hocaoğlu FO, Fidan M, Gerek ÖN. Mycielski approach for wind speed prediction. Energy Conversion and Management. 2009;50(6):1436-1443

[8] Bilir L, Imir M, Devrim Y, Albostan A. An investigation on wind energy potential and small scale wind turbine performance at İncek region-Ankara, Turkey. Energy Conversion and Management. 2015;103:910-923
[9] Justus C, Mikhail A. Height variation of wind speed and wind distributions statistics. Geophysical Research Letters. 1976;3(5):261-264

[10] Nematollahi O, Kim KC. A feasibility study of solar energy in South Korea. Renewable and Sustainable Energy Reviews. 2017;77:566-579

[11] Shin Y, Koo WY, Kim TH, Jung S, Kim H. Capacity design and operation planning of a hybrid PV-wind-batterydiesel power generation system in the case of Deokjeok island. Applied Thermal Engineering. 2015;89:514-525

[12] Ali S, Lee SM, Jang CM. Technoeconomic assessment of wind energy potential at three locations in South Korea using long-term measured wind data. Energies. 2017;10(9):1442 



\title{
Methodology for Sizing Hybrid Battery-Backed Power Generation Systems in Off-Grid Areas
}

\author{
Oswaldo A. Arraez-Cancelliere, Nicolás Muñoz-Galeano \\ and Jesús M. López-Lezama
}

\begin{abstract}
In developing countries, rural electrification in areas with limited or no access to grid connection is one of the most challenging issues for governments. These areas are partially integrated with the electrical grid. This poor electricity distribution is mainly due to geographical inaccessibility, rugged terrains, lack of electrical infrastructure, and high required economic investment for installing large gridconnected power lines over long distances to provide electricity for regions with a low population. On the other hand, rapid depletion of fossil fuel resources on a global scale and progressive increase of energy demand and fuel price are other motives to reduce the reliance on fossil fuels. Hybrid renewable energy system (HRES) can be a suitable option for such remote areas. The objective of this chapter is to develop a methodology for sizing hybrid power generation systems (solardiesel), battery-backed in non-interconnected zones, which minimizes the total cost and maximizes the reliability of supply using particle swarm optimization (PSO). The proposed methodology assists the sizing and designing process of an HRES for an off-grid area minimizing the cost of energy (COE) and maximizing the reliability of the system. Economic incentives offered by the Colombian government are considered in the model.
\end{abstract}

Keywords: hybrid renewable energy system (HRES), stand-alone systems, off-grid areas, particle swarm optimization (PSO), photovoltaic energy, power dispatch strategy

\section{Introduction}

Due to the technological and industrial worldwide progress and the growing industry and society need of power generation for the development and increment of life quality, it is of unquestionable importance to increase sustainable access to electrical energy. In developing countries, there are still many locations without power supply.

Power generation through fossil generators offers a continuous and reliable source of energy making it a very popular option for electrification in off-grid areas. This alternative presents an initial investment cost relatively low compared to other sources of power generation. However, fossil power generators are sized to meet peak demand and have a low performance when the load is quite below to its rated 
capacity. Additionally, operating and maintenance costs are high; the cost of energy (COE) is subject to changes according the national and international fuel markets. In addition, logistical challenges associated with fuel supply in remote areas can cause a significant increase in generation costs [1]. A solution for these disadvantages is the implementation of HRES which includes fossil and other energy sources. For warm and high-average daily radiation levels, photovoltaic solar energy with battery backup represents an attractive complementary source to diesel generation systems. This solution allows the reduction of generation costs and increased system reliability $[2,3]$.

Hybrid systems have shown lower generation costs and greater reliability than dependent systems of a single source of energy [1,2-6]. Each element of the system has to be properly sized to achieve a techno-economic profitability. Therefore, the penetration of renewable energy sources in the energy market depends mainly on the applied sizing methodology to optimize its design [7].

The optimization of these systems could be complex, since many variables are naturally stochastic and linked to the selected location. Examples of these variables are temperature, solar resource, and load profile of the location [8]. Moreover, the optimization technique depends on the selected objective function, which can be oriented in seeking financial gain, increasing system reliability, and reducing the environmental impact [9].

Then, it is necessary to develop a methodology for optimizing the design of HRES that allows the integration of photovoltaic and diesel generation systems, with or without energy storage, allowing to reduce energy costs and maintaining a high reliability in energy supply in off-grid areas. The methodology requires a set of input information linked to the project site, as meteorological and load profile data, and also technical and economic information of the main equipment of the HRES. Then, an optimization process is necessary to determine the best combination of diesel power, PV power, and battery bank capacity. Economic and reliability parameters that support the solution obtained is expected to be presented with the solution.

In the last decade, several optimization techniques have been used to obtain an optimal solution of the sizing of HRES [7, 10-13]. The results among different approaches may vary depending on the characteristics of the model which permits to simulate the behavior of different elements of the system and also the economic and reliability model used as base on the optimization process.

The main objective of this work is to develop an optimization methodology for sizing HRES in off-grid areas of developing countries. In contrast to other works, each step of the methodology is described in detail. Also, special condition will be considered on the development of the economic and reliable model to adjust it to the reality of Colombia, for example, the national and international physical distribution cost or the incentive proposed by the Act 1715 for electrification using nonconventional energy sources in Colombia.

\section{Proposed methodology}

In this methodology, the grid can be formed either from the diesel unit or from a master inverter. The diesel generation is only required when the energy produced by the photovoltaic source and the energy backup in the battery bank is lower than the demanded load. The following items summarize the key characteristics of the dispatch strategy used in this work to model PV-diesel with battery storage systems: (1) the system is considered DC-coupled (Figure 1) and (2) the load following strategy is adopted [1]. The diesel generators are only used to supply the load when 


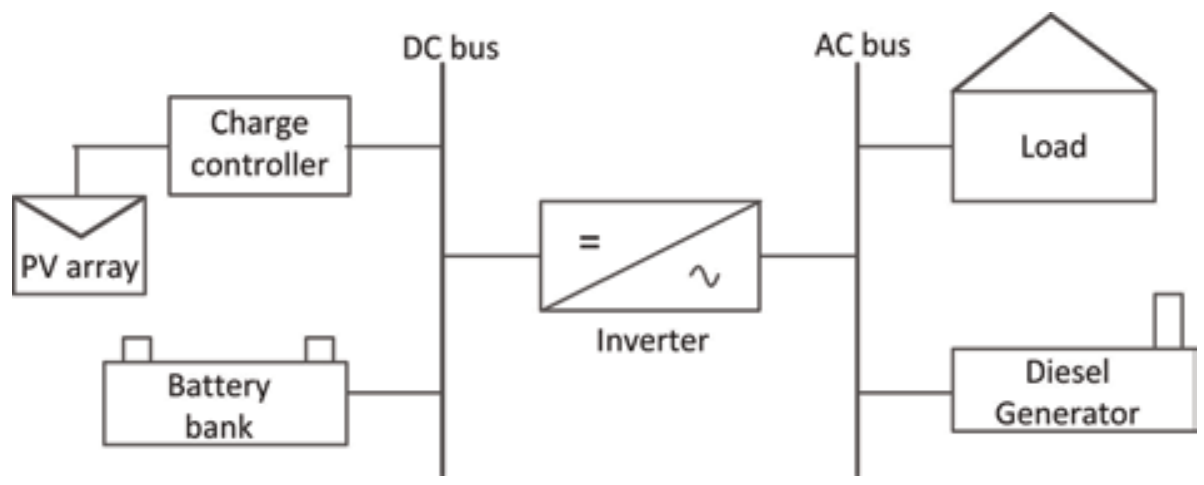

Figure 1.

Schematic diagram of a hybrid solar/battery/diesel generation system.

there is insufficient power from the PV source and the battery bank. Only the minimum DG unit required operates in every time step; (3) all DG units must operate over the minimum load ratio $\left(\delta_{\text {min }}\right)$ defined otherwise the DG unit must be turned off; (4) all DG units have the same nominal power capacity and operate at the equilibrium point at the same load ratio; (5) when diesel units are operating, the PV generation prioritizes the charge of the battery bank over the load; (6) only AC loads are considered; and (7) a maximum number of DG units are considered.

The proposed methodology is composed of the following steps: (1) a dispatch strategy algorithm, (2) calculation of economic indicators, (3) calculation of reliability indicators, (4) calculation of fiscal incentives, and (5) a PSO optimization process given an objective function which optimizes the number of components of the installation and a calculation of economic and reliability indicators for the best solution. The following subsections detail the steps of the methodology. Figure 2 shows the schematic of the proposed methodology and the optimization process.

\subsection{Dispatch strategy algorithm}

Figure 3 shows the dispatch strategy flowchart used on the diesel-PV-battery model for a year which algorithm is described in detail below.

1. Obtain or generate inputs of the system: load profile $\left(P_{L}\right)$, irradiance $(G)$, and temperature $(T)$ for the location in a year. Load profile can be obtained through a survey considering the uncertainties on the input data (38) and (39), and also load profile can also be obtained using measurement of the electrical demand. High-quality solar resource and meteorological data can be obtained by two approaches: high-accuracy instruments installed at a meteorological station and complex solar meteorological models which are validated using high-quality ground instruments.

2. Introduce the following technical information of each element of the system and initialize variables.

2.1. According to the available location and its restrictions, introduce the following technical information: $N_{D G}$, max (maximum number of DG units), $w_{D G}$ (rated power of the available diesel generator), $\delta_{\min }$ (minimum load ratio [\%]), $f_{0}$ (fuel Curve intercept coefficient $[l / k W]$ ), $f_{1}$ (fuel curve slope coefficient $[l / k W]$ ), $N_{p v}$ (number of PV modules), $P_{p v_{s c}}$ (rated power of the 


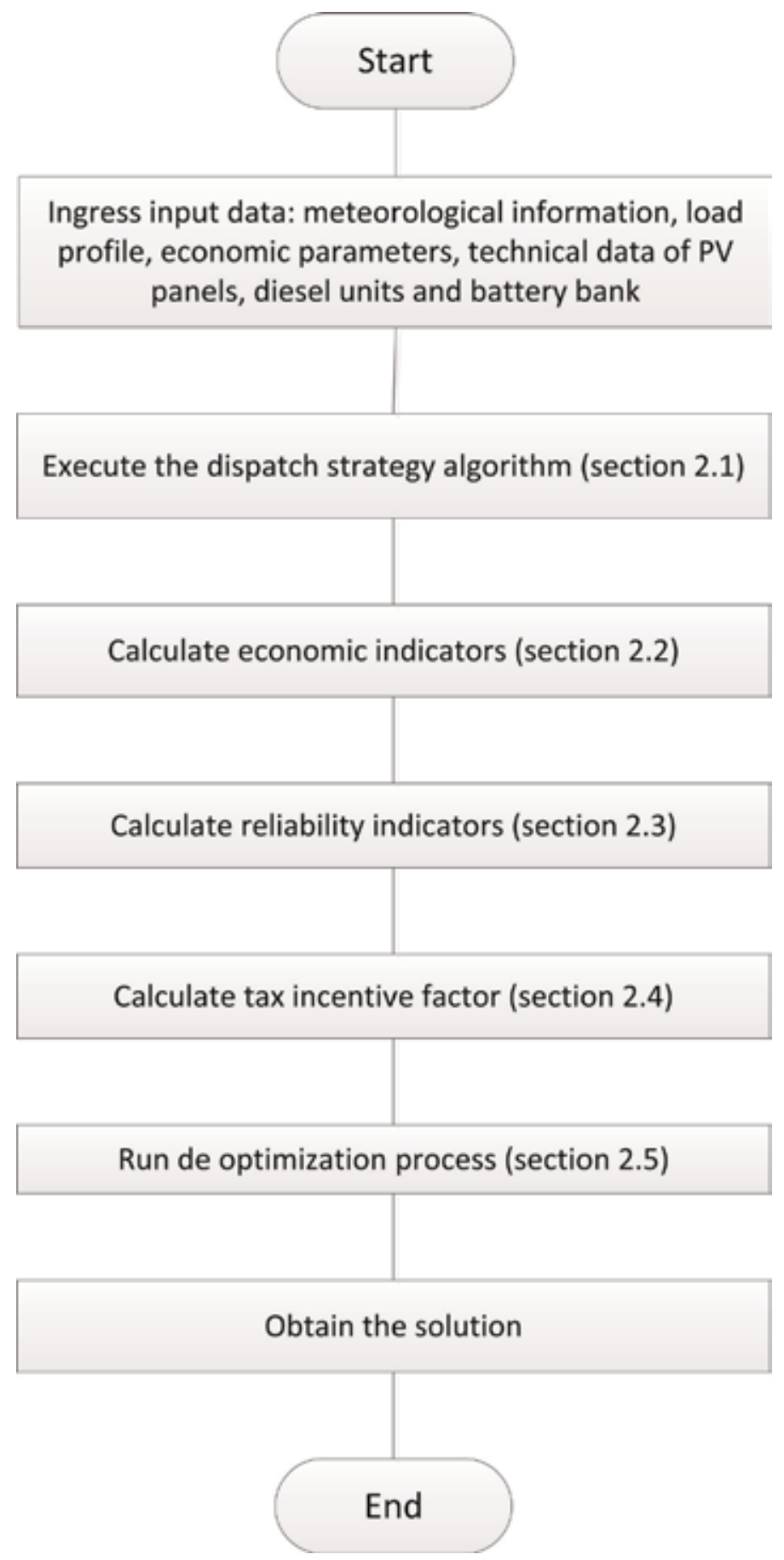

Figure 2.

Schematic diagram of the proposed methodology.

solar module in standard test conditions $[W p]$ ), $G_{s t c}$ (global irradiance in standard test condition $\left.\left[\mathrm{W} / \mathrm{m}^{2}\right]\right), \alpha_{p}$ (temperature coefficient of maximum power $\left[\% /{ }^{\circ} \mathrm{C}\right]$ ), NOCT (nominal operating cell temperature), $T_{s t c}$ (temperature of the cell standard test condition $\left[{ }^{\circ} \mathrm{C}\right]$ ), $T$ (cell's temperature), $f_{p v}$ (derating factor of the solar module), $\eta_{i n v}$ (efficiency of inverters), $N_{b p}$ 


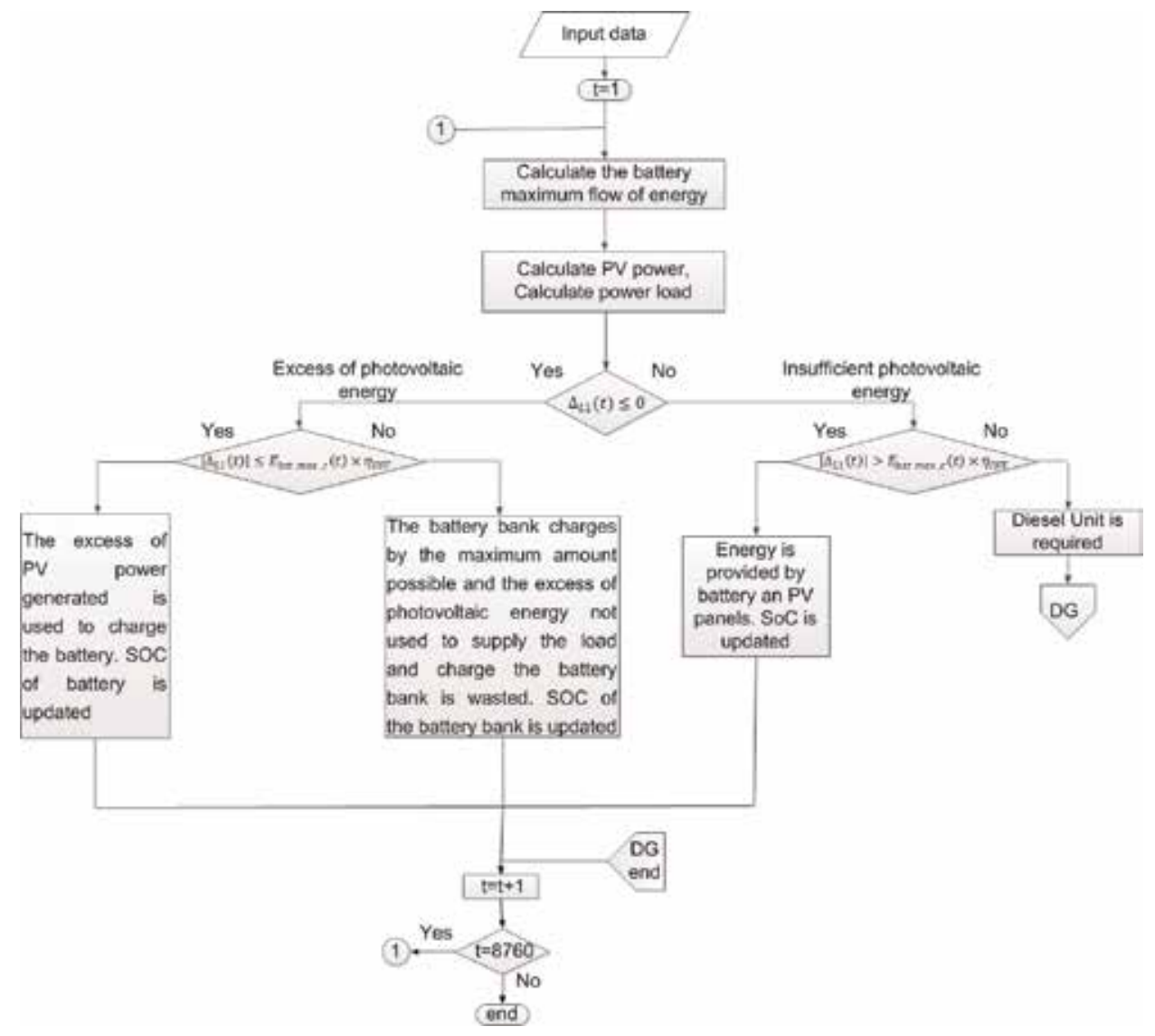

Figure 3.

Dispatch strategy flowchart.

(number of batteries in parallel), $E_{\text {bcell, nom }}$ (nominal capacity of one battery cell $[k W h]$ ), $V_{d c_{s i t}}$ (DC voltage system [V]), $E_{\max }$ (maximum flow of energy to charge or discharge the battery bank $[k W h]$ ), $V_{d c_{b c}}$ (nominal voltage of each battery cell), $\eta_{b a t, d}$ (discharge efficiency of the battery), $\eta_{b a t, c}$ (charge efficiency of the battery), $C_{\text {rate }}$ (capacity rate), $D O D_{\max }$ (maximum deep of discharge of the battery bank [\%]), $\sigma$ (self-discharge coefficient), $w_{D G}$ (diesel rated power), and $\delta_{\text {min }}$ (diesel minimum load ratio).

2.2. Initialize the following variables: $\Delta_{L 1}=0$ (difference between PV energy generated and the energy demanded by the load), $t=1$ (initial time instant, first hour of the year), $S O C(1)=S O C_{\max }$ (state of charge $(S O C)$ is initialized considering that the battery is full charged), ENS $=0$ (energy not supplied), $P F T=0$ (power time failure, $E W=0$ (energy wasted), $P_{D G}=0$ (diesel output power), $F C_{D G}=0$ (consumption of the diesel generator, $N_{o n}=0$ (number of DG on), and $\delta=0$ (diesel load ratio).

3. Calculate the battery model which expresses the equations in the function of the energy each hour:

(1) The maximum amount of energy that the battery bank can be discharged in one time step $\left(E_{b a t, \max , d}(t)[k W h]\right)$ is as follows: 


$$
E_{\text {bat }, \max d}(t)=\max \left[0, \min \left[E_{\text {max }},\left(S O C(t)-S O C_{\text {min }}\right)\right]\right]
$$

(2) The maximum amount of energy that the battery can be charged in one time step $\left(E_{b a t, \max , c}(t)[k W h]\right)$ is as follows:

$$
E_{\text {bat }, \max _{-} c}(t)=\max \left[0, \min \left[E_{\text {max }},\left(S O C_{\text {max }}-S O C(t)\right)\right]\right]
$$

4. Calculate the hourly generated energy of the PV system $\left(P_{p v}(t)[k W h]\right)$. The $\mathrm{PV}$ power output for time step $\mathrm{t}$ is calculated using [14]:

$$
P_{p v}(t)=N_{p v} \times P_{p v_{s c}} \times \frac{G(t)}{G_{s t c}} \times\left(1+\frac{\alpha_{p}}{100} \times\left(T(t)-T_{s t c}\right)\right) \times f_{p v}
$$

5. Calculate the difference between PV energy generated and the energy demanded by the load $\left(\Delta_{L 1}(t)\right)$ :

$$
\Delta_{L 1}(t)=P_{L}(t)-P_{p v}(t) \times \eta_{i n v}
$$

6. If $\Delta_{L 1}(t) \leq 0$, then the PV source can supply the load.

6.1. If $\left|\Delta_{L 1}(t)\right| \leq E_{\text {bat. max }, c}(t) \times \eta_{I N V}$, the excess of PV energy generated $\left(E_{b a t}(t)\right)$, if any, is used to charge the battery bank, and the SOC of the battery is updated:

$$
\begin{gathered}
E_{b a t}(t)=P_{p v}(t)-\frac{P_{L}(t)}{\eta_{I N V}} \\
S O C(t+1)=S O C(t) \times(1-\sigma)+E_{b a t}(t) \times \eta_{b a t, c}
\end{gathered}
$$

Go to step 10 .

6.2. Else, the battery bank is fully charged; SOC is updated. There is excess of energy that cannot be used supplying the load or charging the battery, so energy wasted $(E W)$ is calculated.

$$
\begin{gathered}
E_{\text {bat }}(t)=E_{\text {bat. max }, c}(t) \\
S O C(t+1)=S O C(t) \times(1-\sigma)+E_{b a t}(t) \times \eta_{b a t, c} \\
E W(t)=P_{p v}(t)-\frac{P_{L}(t)}{\eta_{I N V}}-E_{b a t}(t)
\end{gathered}
$$

Go to step 10 .

7. If $\Delta_{L 1}(t)>0$, the photovoltaic source is insufficient to supply the load.

7.1. If $\Delta_{L 1}(t)<E_{b a t, \max , d}(t) \times \eta_{I N V}$, the battery bank discharge to supply the lack of energy. SOC of the battery is updated.

$$
\begin{gathered}
E_{b a t}(t)=\frac{P_{L}(t)}{\eta_{I N V}}-P_{p v}(t) \\
S O C(t+1)=S O C(t) \times(1-\sigma)-E_{b a t}(t) \times \eta_{b a t, d}
\end{gathered}
$$

Go to step 10 . 
7.2. Otherwise, diesel generation is required. Go to step 8 .

8. Diesel generation is necessary. Photovoltaic energy is used to charge the battery bank, and the diesel generation is used to supply the load. The energy stored in the battery bank and energy generated by the diesel unit is used to supply the load at night.

8.1. Case 1: $P_{L}(t)<\delta_{\min } \times w_{D G}$. Since the DG units cannot operate under the minimum load ratio, $\delta_{\text {min }}$, all DG units must be turned off $\left(N_{o n}(t)=0\right.$, $\left.\delta(t)=0 ; P_{D G}(t)=0\right)$. The generated PV energy and the energy available in the battery bank are used to supply the load, while the energy not supplied (ENS) and the power time failure (PTF) are counted:

$$
\begin{gathered}
E_{\text {bat }}(t)=E_{\text {bat. max }, d} \\
\operatorname{SOC}(t+1)=\operatorname{SOC}(t) \times(1-\sigma)-E_{\text {bat }}(t) \times \eta_{b a t \_d} \\
\operatorname{ENS}(t)=P_{L}(t)-\left(P_{p v}(t)+E_{b a t}(t)\right) \times \eta_{i n v} \\
P F T=P F T+1
\end{gathered}
$$

Go to step 10 .

8.2. Case 2: $P_{L}(t) \geq \delta_{\min } \times w_{D G} \& \& P_{p v}(t)>0$. The photovoltaic energy is used to charge the battery bank. The diesel generation supplies the load.

8.2.1. Case 2.1: $P_{p v}(t) \geq E_{\text {bat. } \max , c}(t)$. The battery bank charges at its maximum ratio, and the excess of energy is used to supply the load with the diesel generation.

$$
\begin{gathered}
E_{b a t}(t)=E_{\text {bat. max }, c}(t) \\
\operatorname{SOC}(t+1)=\operatorname{SOC}(t) \times(1-\sigma)+E_{b a t}(t) \times \eta_{b a t, c} \\
P_{D G}(t)=\min \left(N_{d g, \max } * w_{d g}, P_{L}(t)-\left(P_{p v}-E_{b a t}(t)\right) \times \eta_{i n v}\right. \\
N_{o n}(t)=\left\lceil\frac{P_{D G}(t)}{w_{d g}}\right\rceil \\
\delta(t)=\frac{P_{D G}(t)}{N_{o n}(t) \times w_{D G}}
\end{gathered}
$$

Go to step 9.

8.2.1.1. Case 2.1.1: $P_{L}(t)>\left(P_{p v}(t)-E_{b a t}(t)\right) \times \eta_{i n v}-P_{D G}(t)$. Diesel generation is not sufficient to supply the load; the energy not supplied is accounted:

$$
\begin{gathered}
E N S(t)=P_{L}(t)-\left(P_{p v}(t)-E_{b a t}(t)\right) \times \eta_{i n v}-P_{D G}(t) \\
P F T=P F T+1
\end{gathered}
$$

Go to step 9.

8.2.1.2. Case 2.1.2: $\delta(t)<\delta_{\text {min }}$. If the load ratio of the DG unit is lower than the minimum load ratio allowed, then just one DG unit $\left(N_{o n}(t)=1\right)$ works operating at the minimum load ratio $\left(\delta(t)=\delta_{\min }\right)$, and the excess of PV energy generated is wasted: 


$$
\begin{gathered}
P_{D G}(t)=N_{o n}(t) \times \delta(t) \times w_{D G} \\
E W(t)=P_{p v}(t)-E_{b a t}(t)-\frac{P_{L}(t)-P_{d g}(t)}{\eta_{i n v}}
\end{gathered}
$$

Go to step 9.

8.2.2. Case 2.2: $P_{p v}(t) \leq E_{\text {bat. max }, c}(t)$. All photovoltaic energy is used to charge the battery bank:

$$
\begin{gathered}
E_{b a t}(t)=P_{p v}(t) \\
P_{D G}(t)=\min \left(N_{d g}, \max * w_{d g}, P_{L}(t)\right. \\
S O C(t+1)=S O C(t) \times(1-\sigma)+E_{b a t}(t) \times \eta_{b a t, c} \\
N_{o n}(t)=\left\lceil\frac{P_{D G}(t)}{w_{d g}}\right\rceil \\
\delta(t)=\frac{P_{D G}(t)}{N_{o n}(t) \times w_{D G}}
\end{gathered}
$$

8.2.2.1. Case 2.2.1: $P_{L}(t)>P_{D G}(t)$. The DG is insufficient to supply the load; the energy not supplied is accounted:

$$
\begin{gathered}
E N S(t)=P_{L}(t)-P_{D G}(t) \\
P F T=P F T+1
\end{gathered}
$$

8.3. Case 3: $\left(P_{L}(t) \geq \delta_{\text {min }} \times w_{D G} \& \& P_{p v}(t) \leq 0\right)$. At night, the battery bank and the DG units are used to supply the load.

8.3.1. Case 3.1: $\left(P_{L}(t)-E_{\text {bat. } \max , d}(t) \times \eta_{\text {inv }} \geq \delta_{\text {min }} \times w_{D G}\right)$. Battery bank is discharged at maximum rate, and DG units generate the remaining energy necessary to supply the load.

$$
\begin{gathered}
E_{\text {bat }}(t)=E_{\text {bat.max }, d}(t) \\
P_{D G}(t)=\min \left(N_{d g, \text { max }} * w_{d g}, P_{L}(t)-E_{b a t}(t) \times \eta_{\text {inv }}\right. \\
S O C(t+1)=S O C(t) \times(1-\sigma)-E_{\text {bat }}(t) \times \eta_{\text {bat }, d} \\
N_{\text {on }}(t)=\left\lceil\frac{P_{D G}(t)}{w_{d g}}\right\rceil \\
\delta(t)=\frac{P_{D G}(t)}{N_{o n}(t) \times w_{D G}}
\end{gathered}
$$

8.3.1.1. Case 3.1.1: $\left(P_{L}(t) \geq P_{D G}+E_{b a t}(t) \times \eta_{i n v}\right)$. The diesel generation and the energy provided by the battery bank are not sufficient to supply the load; the energy not supplied is accounted.

$$
\begin{gathered}
E N S(t)=P_{L}(t)-P_{D G}(t)-E_{b a t}(t) \times \eta_{i n v} \\
P F T=P F T+1
\end{gathered}
$$

8.3.2. Case 3.2: $\left(P_{L}(t)-E_{\text {bat. max }, d}(t) \times \eta_{i n v}<\delta_{\text {min }} \times w_{D G}\right)$. Just one DG unit works operating at the minimum load ratio $\left(N_{o n}(t)=1, \delta(t)=\delta_{\text {min }}\right)$. The battery bank provides the insufficient energy to supply the load. 


$$
\begin{gathered}
P_{D G}(t)=N_{o n}(t) \times \delta(t) \times w_{D G} \\
E_{b a t}(t)=\frac{P_{L}(t)-P_{D G}(t)}{\eta_{i n v}} \\
S O C(t+1)=S O C(t) \times(1-\sigma)-E_{b a t}(t) \times \eta_{b a t, d}
\end{gathered}
$$

9. The fuel consumption $F C_{D G}(t)$ is calculated by $[15,16]$ :

$$
F C_{D G}(t)=N_{o n}(t) \times w_{d g} \times f_{0}+P_{D G}(t) \times f_{1}
$$

10. Increase the time step $(t=t+1)$. If $t \leq 8760$, and return to step 3. Else END.

After run the previous algorithm; economic and reliability indicators should be calculated using the following procedure.

\subsection{Economic indicators}

An economic analysis is required to determine the optimum cost and benefit ratio of HRES. These systems generally require high capital investment, even though they have low operation and maintenance (O\&M) costs and less fuel costs in comparison with systems relaying only on fossil fuels. In this study, the annualized cost of the system (ACS) and the cost of energy (COE) are considered as the economic criteria to evaluate the feasibility of this hybridized system configuration.

The annualized cost of the system (ACS) is the sum of the annualized capital cost $(C C)$, the annualized replacement cost $(R C)$ and the annualized cost of maintenance $(O M)$ [7, 17-19]. In [17], the annualized cost of the system is defined as

$$
\left.A C S=\sum_{i=1}^{N_{c}}\left(C C_{i}+R C_{i}\right) \times C R F\left(i_{r}, R\right)+O \& M_{i}\right)
$$

where $N_{c}$ is the number of components; in this study there are three components (PV modules, battery banks, DG units). Subscript $i$ is used to describe the cost of each component. The capital recovery factor $\left(C R F\left(i_{r}, R\right)\right)$ can be defined as a ratio used to calculate the present value of an annuity (a series of equal annual cash flows) in the function of the real interest rate $\left(i_{r}\right)$ and the lifetime of the project $(R)$ [17]. The capital recovery factor is calculated by

$$
C R F(i r, R)=\frac{i_{r} \times\left(1+i_{r}\right)^{R}}{\left(1+i_{r}\right)^{R}-1}
$$

The real interest rate is used to convert between one-time costs and annualized costs. By defining the real discount rate, the inflation rate effect is factored out of the economic analysis. All costs, therefore, become real costs, which are in defined in terms of constant dollars. The real interest rate is calculated by

$$
i_{r}=\frac{i_{n}-i_{f}}{1+i_{f}}
$$

where $i_{n}$ and $i_{f}$ are the nominal interest rate and expected annual inflation rate, respectively.

The capital cost for each component is described as follows:

$$
C C_{p v}=c_{p v} \times N_{p v} \times P_{p v_{s t c}}
$$




$$
\begin{gathered}
C C_{b a t}=c_{b a t} \times N_{b a t} \times E_{b c e l l, \text { nom }} \\
C C_{D G}=c_{D G} \times N_{D G} \times w_{D G}
\end{gathered}
$$

where $c_{p v}$ is the cost per Watt peak installed of photovoltaic power in [USD/ $\mathrm{Wp}$ ]; this cost includes the cost of the module, the electronic power equipment required (charge controller and inverter), and the installation cost (engineering, transportation, balance of system equipment as cable, mounting rack, electrical protection, etc.). $c_{p v}$ varies according to the project location and site conditions; it can range from 3 to $10 \mathrm{USD} / \mathrm{Wp}$. The cost per unit of the battery system, $c_{b a t}$, in [USD/Wh], includes the average cost of the battery cell and the installation cost of the battery system. The parameter $c_{D G}$ in [USD/kW] is the cost per unit of diesel generation installed and also includes the cost of the diesel generator unit and the associated installation costs.

The replacement cost is calculated for each element. The replacement cost of the photovoltaic system is assumed null, as the photovoltaic modules have a life cycle superior to the lifetime of the project and it is assumed in this model that the charge controllers and inverters do not need replacement during the lifetime of the project. The replacement cost of the battery system and the DG unit can be calculated as

$$
\begin{gathered}
R C_{b a t}=\gamma_{b a t} \times C C_{b a t} \times K_{b a t}\left(i_{r}, L_{p v}, y_{i}\right) \\
R C_{D G}=\gamma_{D G} \times C C_{D G} \times K_{D G}\left(i_{r}, L_{D G}, y_{i}\right)
\end{gathered}
$$

where $\gamma_{\mathrm{bat}}$ and $\gamma_{\mathrm{DG}}$ are derate factors of the initial capital cost invested for the battery system and the diesel genset, respectively, as some cost necessary during the installation are no longer needed during the replacement (civil works, battery rack, electrical protections, fuel tank, etc.). $K_{i}\left(i_{r}, L_{i}, y_{i}\right)$ is the single payment present worth [17], which is defined by

$$
K_{i}\left(i_{r}, L_{i}, y_{i}\right)=\sum_{n=1}^{y_{i}} \frac{1}{\left(1+i_{r}\right)^{n \times L_{i}}}
$$

where $L$ and $y$ are the useful lifetime and the number of replacements of the component during the lifetime of the project, respectively. The number of replacements of each component is a function of useful lifetime of the component and the lifetime of the project $\left(y_{i}=\left\lfloor\frac{R}{L i}\right\rfloor\right)$.

The fixed mount PV systems do not have moving parts, so operating and maintenance costs consist of regular cleaning and monitoring of performance, the annual operation, and maintenance cost can be estimated as a percentage of the PV system total investment, $\rho_{p v}$, usually between 1 and $2 \%$ [20].

$$
O \& M_{P V}=\rho_{p v} \times C C_{p v}
$$

In a similar way, the annual operation and maintenance cost for the battery system can be calculated as percentage of the total investment cost of the battery system. This cost can vary according to the technology of the battery bank. For example, the cost of operation and maintenance for vented lead-acid batteries is higher than maintenance-free sealed lead-acid batteries or Li-ion batteries. The percentage of the total investment cost, $\rho_{\text {bat }}$, can vary between 1 and $3 \%$.

$$
O \& M_{b a t}=\rho_{b a t} \times C C_{b a t}
$$

The operation and maintenance cost for the diesel system components is divided in two values: a fixed cost, expressed as a percentage of the diesel initial investment, 
$\rho_{D G}$, and a variable cost associated to the cost of fuel, $f_{C}$, in [\$/gal], and the annual fuel consumption. The annual operation and maintenance cost of the diesel system can be calculated by

$$
O \& M_{D G}=\rho_{D G} \times C C_{D G}+f_{C} \times \sum_{t=1}^{8760} F C(t)
$$

The cost of energy (COE) can be defined as the average cost per kWh of useful electrical energy produced by the system [21]. It can be obtained as the ratio between the annualized cost of the system and the effective load served in 1 year. The economic model assumes that the yearly effective load served is constant over the lifetime of the project. COE can be calculated as follows:

$$
C O E=\frac{A C S}{\sum_{t=1}^{8760}\left(E_{L}(t)-E N S(t)\right)}
$$

\subsection{Reliability indicators}

The dependency on nature and unpredictability of solar resources has a great impact on energy production which leads to unreliable power supply during cloudy days. A system is reliable if it can supply the required power to the electrical load within a specific time period.

The loss of power supply probability (LPSP) is the most widely used method to evaluate the reliability in hybrid system, therefore is selected, in this work, as reliability criteria. The LPSP be calculated as the ratio of power supply deficit to the electric load demand during a certain period of time (normally a year). A ratio equal to zero means all load demand, during the period of time, is served by system (53). LPSP is given by

$$
L P S P=\frac{\sum_{t=1}^{8760} \operatorname{ENS}(t)}{\sum_{t=1}^{8760} E_{L}(t)}
$$

A method that takes into account the weight of reliability in the economic model includes a component of the cost of electricity interruptions or cost of load $\left(C_{\text {loss }}\right)$ [17]. The cost of electricity interruptions can be estimated in different ways, for example, looking at the customer's willingness to pay for an expansion or at production losses at industries affected, or at the level of compensations, which makes shortages acceptable. In [17], for 2009, the cost ranges from 5 to 40 USD $\$ / k W h$ for industrial users and 2-12 USD $\$ / \mathrm{kWh}$ for domestic users.

The cost of electricity lost for non-interconnected zone can vary with respect the reference cost and could be difficult to estimate, as depends on the willingness of users to pay for a more robust system. The cost of electricity not supply $\left(C_{\text {loss }}\right)$ in [USD/kWh] is an input parameter in the economic model. The annualized cost of energy not supplied can be calculated as

$$
A C_{\text {loss }}=C_{\text {loss }} \times \sum_{t=1}^{8760} E N S(t)
$$

LPSP and $A C_{\text {loss }}$ are calculated for each possible combination considered during the sizing methodology. 


\subsection{Fiscal incentives}

Under the Colombian Renewable Energy Law, new clean energy projects will receive up to $50 \%$ tax credits, but they can only be applied during the first 5 years. In this work, when the fiscal incentives are considered, it is assumed that the company will receive the $50 \%$ of the tax credit equally distributed over the first 5 years of the project. In general, investment tax credits can be calculated as

$$
\begin{gathered}
i=\sum_{j=1}^{5} i_{j}=0.5 \\
i_{1}=i_{2}=i_{3}=i_{4}=i_{5}=0.1
\end{gathered}
$$

In a similar way, it is assumed that the effect of depreciation is equally distributed each year, and the useful life for accelerated depreciation purposes is 5 years; then

$$
\begin{gathered}
d=\sum_{j=1}^{5} d_{j}=1 \\
d_{1}=d_{2}=d_{3}=d_{4}=d_{5}=0.2
\end{gathered}
$$

Assuming an effective corporate tax income rate of $33 \%$ and under the previous consideration, the tax reduction factor $\Delta$ for the purpose of this work is given by

$$
\Delta=\frac{1}{(1-t)} \times\left[1-t \times\left(\sum_{j=1}^{T 1} \frac{i_{j}}{\left(1+i_{r}\right)^{j}}+\sum_{j=1}^{T 2} \frac{d_{j}}{\left(1+i_{r}\right)^{j}}\right)\right]
$$

wheret is the effective corporate tax income rate, $T 1$ is the maximum number of years to apply the investment tax credit, $T 2$ is the useful life of the powergenerating facility for accelerated depreciation purposes (in year) $=5, i$ is the investment tax credit, and $d$ is the depreciation factor expressed as percentage of investment cost over $T 2$ year.

Fiscal incentives granted by the Colombian Act 1715 only apply to not conventional energy source installation and its components. In this way, the incentive tax factor only applies to the capital cost of photovoltaic and battery components:

$$
\begin{aligned}
A C S_{a d j}= & {\left[\left(C C_{p v}+C C_{b a t}\right) \times \Delta+C C_{D G}+R C_{b a t}+R C_{D G}\right] \times C R F\left(i_{r}, R\right)+O \& M_{p v} } \\
& +O \& M_{b a t}+O \& M_{D G}
\end{aligned}
$$

\subsection{Objective function: optimization process}

The objective of this work is sizing hybrid power generation systems (solardiesel) battery-backed, in non-interconnected zones, which minimizes the total cost of the solution and maximize the reliability of supply. To minimize the total cost of the system, the following objective function is used:

$$
\text { Cost }=\frac{A C S_{a d j}+A C_{\text {loss }}}{\sum_{t=1}^{8760}\left(E_{L}(t)-E N S(t)\right)}
$$

This work aims to develop an optimization model for sizing an energy system to supply the energy demand on an off-grid location. The optimization of these 
systems could be complex, since many variables are naturally stochastic depending mostly on the characteristic of the solar resource and the load profile of the selected location. The objective is to minimize the total cost of the solution and maximize the reliability of the supply.

As a result of the optimization problems, the following information are obtained: (1) amount of photovoltaic modules and therefore the total photovoltaic power in $\mathrm{kWp},(2)$ amount of diesel generation units and the total diesel energy power in $\mathrm{kWp}$, (3) amount of battery cell required and total capacity of the energy storage system in $\mathrm{kWh}$, (4) energy flow in the system showing the different states of the system according to the dispatch strategy described in this work, (5) discriminated cost of each technology in terms of initial capital required and O\&M cost, (6) annualized cost of energy of the best solution, and (7) amount and cost of energy not supplied and LPSP.

\section{Case study}

"Santa Cruz del Islote" in Bolivar, Colombia, was used as a location for the case study. This rural community is selected to evaluate the optimization model developed in this work.

\subsection{Meteorological inputs and load profile}

The monthly global irradiance over the horizontal and over the plane of the array was calculated using a MATLAB routine developed in this work and then compared with results obtained from Solargis. Table 1 shows the results obtained.

\begin{tabular}{ccccccc}
\hline & $\begin{array}{c}\text { Global } \\
\text { horizontal } \\
\text { irradiation } \\
{\left[\mathbf{k W h} / \mathbf{m}^{2}\right]} \\
\text { Solargis }\end{array}$ & $\begin{array}{c}\text { Global horizontal } \\
\text { irradiation } \\
{\left[\mathbf{k W h} / \mathbf{m}^{2}\right]}\end{array}$ & $\begin{array}{c}\text { Dev } \\
\text { calculated }\end{array}$ & $\begin{array}{c}\text { Global tilted } \\
\text { irradiation } \\
{\left[\mathbf{k W h} / \mathbf{m}^{2}\right]} \\
\text { Solargis }\end{array}$ & $\begin{array}{c}\text { Global tilted } \\
\text { irradiation } \\
{\left[\mathbf{k W h} / \mathbf{m}^{2}\right]}\end{array}$ & $\begin{array}{c}\text { Dev } \\
\text { [\%] }\end{array}$ \\
\hline Jan & 183.6 & 182.0 & $-0.88 \%$ & 201.9 & 198.6 & $-1.65 \%$ \\
\hline Feb & 175.6 & 174.2 & $-0.81 \%$ & 186.9 & 184.3 & $-1.41 \%$ \\
\hline Mar & 194.3 & 193.0 & $-0.68 \%$ & 198.5 & 196.2 & $-1.14 \%$ \\
\hline Apr & 177.2 & 176.1 & $-0.65 \%$ & 175 & 172.9 & $-1.17 \%$ \\
\hline May & 166.4 & 165.2 & $-0.70 \%$ & 160.1 & 158.8 & $-0.83 \%$ \\
\hline Jun & 161.9 & 160.8 & $-0.71 \%$ & 153.6 & 152.6 & $-0.65 \%$ \\
\hline Jul & 173.2 & 172.0 & $-0.69 \%$ & 165.3 & 163.9 & $-0.84 \%$ \\
\hline Aug & 171.7 & 170.6 & $-0.65 \%$ & 167.8 & 166.1 & $-1.03 \%$ \\
\hline Sep & 160.9 & 159.8 & $-0.70 \%$ & 162 & 160.1 & $-1.17 \%$ \\
\hline Oct & 155.8 & 154.4 & $-0.91 \%$ & 162.4 & 159.5 & $-1.79 \%$ \\
\hline Nov & 149.1 & 147.7 & $-0.96 \%$ & 160.5 & 157.1 & $-2.13 \%$ \\
\hline Dec & 161.2 & 159.7 & $-0.93 \%$ & 177.8 & 174.1 & $-2.09 \%$ \\
\hline Year & 2030.9 & 2015.3 & $-0.77 \%$ & 2071.8 & 2044.1 & $-1.34 \%$ \\
\hline
\end{tabular}

Table 1.

Meteorological input parameters (monthly). 
The difference can be accounted to the simplicity of the transposition model used in our MATLAB routine; nevertheless the results are good enough for the purpose of this work.

The load profile data was obtained from the National Monitoring Center (CNM) of the IPSE [22]. Table 2 shows the input data used to generate the daily load profile curve. Figure 4 shows the daily load profile for a week generated by a MATLAB routine developed in this work.

\subsection{Technical inputs}

This subsection describes the technical inputs required by the photovoltaic, diesel, and battery model employed in the optimization model developed in this work.

\begin{tabular}{cccccc}
\hline Hour & Power [\%] & Uncertainty factor [\%] & Hour & Power [\%] & Uncertainty factor [\%] \\
\hline $\boldsymbol{h}$ & $\boldsymbol{\alpha}_{\text {power }}$ & $\boldsymbol{\alpha}_{\text {unc }}$ & $\boldsymbol{h}$ & $\boldsymbol{\alpha}_{\text {power }}$ & $\boldsymbol{\alpha}_{\text {unc }}$ \\
\hline 0 & 7.78 & 10 & 12 & 0.96 & 10 \\
\hline 1 & 7.68 & 10 & 13 & 2.88 & 10 \\
\hline 2 & 7.40 & 10 & 14 & 5.67 & 10 \\
\hline 3 & 7.20 & 10 & 15 & 5.86 & 10 \\
\hline 4 & 6.34 & 10 & 16 & 3.75 & 10 \\
\hline 6 & 1.15 & 10 & 17 & 1.54 & 10 \\
\hline 7 & 0.00 & 0 & 18 & 0.96 & 10 \\
\hline 8 & 0.00 & 0 & 19 & 6.24 & 10 \\
\hline 9 & 0.00 & 0 & 20 & 8.65 & 10 \\
\hline 10 & 0.00 & 0 & 21 & 8.65 & 10 \\
\hline 11 & 0.00 & 0 & 22 & 8.65 & 520.5 \\
\hline
\end{tabular}

Table 2.

Daily load profile for "Santa Cruz del Islote" July 2018.

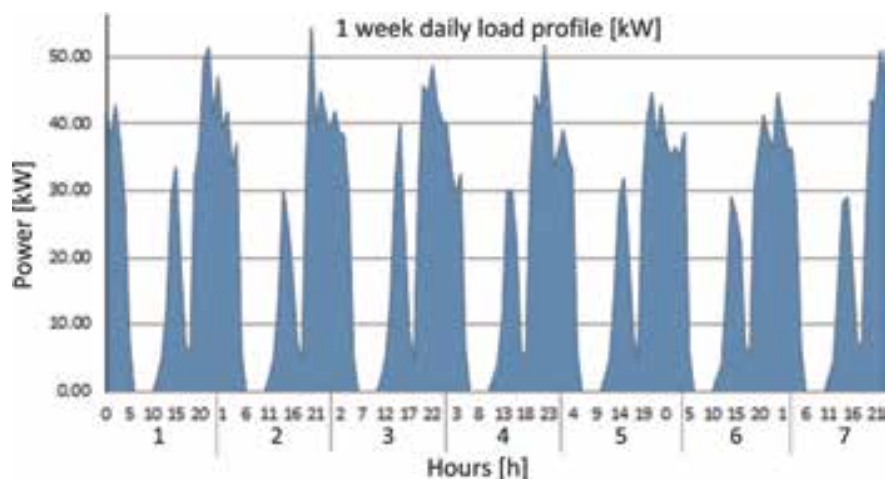

Figure 4.

Daily load profile for a week generated for "Santa Cruz del Islote." 


\subsubsection{Photovoltaic module technical data}

A monocrystalline PV module of $300 \mathrm{Wp}$, reference JKM300M-60, from the company JINKO SOLAR, is used. Table 3 shows the technical characteristics of the PV module selected. The cost per Wp installed presented in Table 3 includes other costs not related to the price of the PV modules as the cost of charge controller, the $\mathrm{PV}$ inverters, and the mounting structure. Also this price includes indirect cost associated to the PV installation as engineering study costs, logistic costs, and certification costs. The cost per Wp presented is taken as reference and is provided by experts consulted in companies of energy sector.

\subsubsection{Diesel genset technical data}

The input data required by the diesel generation model is presented in Table 4 . This information is collected from expert opinions on companies in the energy

\begin{tabular}{lcc}
\hline Symbol & Description & Value \\
\hline$P_{p v_{s t c}}$ & Maximum power [Wp] & 300 \\
\hline$V_{m p p}$ & Maximum power voltage [V] & 32.6 \\
\hline$I_{m p p}$ & Maximum power current [A] & 9.21 \\
\hline$V_{o c}$ & Open-circuit voltage [V] & 40.1 \\
\hline$I_{s c}$ & Short-circuit current $[\mathrm{A}]$ & 9.72 \\
\hline$\eta_{p v}$ & Module efficiency $(\%)$ & 18.33 \\
\hline$\alpha_{P}$ & Power temperature coefficient $\left[\% /{ }^{\circ} \mathrm{C}\right]$ & -0.39 \\
\hline$\alpha_{V}$ & $\mathrm{~V}_{\text {oc }}$ temperature coefficient $\left[\% /{ }^{\circ} \mathrm{C}\right]$ & -0.29 \\
\hline$\alpha_{I}$ & $\mathrm{I}_{\mathrm{sc}}$ temperature coefficient $\left[\% /{ }^{\circ} \mathrm{C}\right]$ & 0.05 \\
\hline$N O C T$ & NOCT [ $\left.{ }^{\circ} \mathrm{C}\right]$ & 45 \\
\hline$c_{P V}$ & Cost per Wp installed [USD $/ \mathrm{Wp}]$ & 2 \\
\hline$\rho_{P V}$ & Fixed OM factor as ratio of the PV CC & 0.01 \\
\hline$f_{P V}$ & Photovoltaic derating factor & 0.85 \\
\hline$\eta_{I N V}$ & Inverter efficiency & 0.9 \\
\hline
\end{tabular}

Table 3.

PV module technical inputs.

\begin{tabular}{lcc}
\hline & Diesel input data & \\
\hline Symbol & Description & Value \\
\hline$N_{D G, \max }$ & Maximum number of DG unit & 5 \\
\hline$\delta_{\min }$ & Minimum load ratio allowed & 0.3 \\
\hline$L_{D G}$ & Lifecycle [years] & 10 \\
\hline$\rho_{D G}$ & Fixed OM value as percentage of the diesel initial investment [\%] & 0.1 \\
\hline$f_{C}$ & Fuel cost [USD/l] & 0.8 \\
\hline
\end{tabular}

Table 4.

Diesel model technical inputs. 
sector. This information must be validated each time the optimization model is used since it can vary depending on the studied case.

Table 5 shows a database of diesel generation units with the cost per $\mathrm{kW}$ and the fuel curve parameters. This table was built using information supplied by the Colombian Regulation Commission of Energy and Gas (CREG-Comisión de Regulación de Energía y Gas) in [23]. The cost per kW presented in Table 5 includes the direct and indirect costs related to the installation of a Diesel plant in non-interconnected zones.

\begin{tabular}{lcccccc}
\hline $\begin{array}{l}\text { DG } \\
\text { power } \\
{[\mathbf{k W}]}\end{array}$ & $\begin{array}{c}\text { Cost per } \mathbf{k W} \\
\text { installed [USD/ } \\
\mathbf{k W}]\end{array}$ & $\begin{array}{c}\text { Derate factors of the } \\
\text { initial capital cost } \\
\text { invested }[\%]\end{array}$ & $\begin{array}{c}\mathbf{1 / 2} \text { load } \\
\mathbf{1} \text { hour } \mathbf{i n} \\
\text { liters }\end{array}$ & $\begin{array}{c}\text { Full-load } \\
\mathbf{1} \text { hour in } \\
\text { liters }\end{array}$ & $\begin{array}{c}\mathbf{f}_{\mathbf{o}}[\mathbf{L} / \\
\mathbf{k W h} \text { ] }\end{array}$ & $\begin{array}{c}\mathbf{f}_{\mathbf{1}}[\mathbf{L} / \\
\mathbf{k W h}]\end{array}$ \\
\hline 10 & 2724.09 & 31.83 & 1.4 & 2.6 & 0.020 & 0.240 \\
\hline 20 & 1697.26 & 32.43 & 3.4 & 6.05 & 0.037 & 0.265 \\
\hline 25 & 1540.12 & 31.63 & 3.6 & 6.4 & 0.032 & 0.224 \\
\hline 30 & 1934.44 & 23.00 & 6.8 & 10.96 & 0.088 & 0.277 \\
\hline 40 & 1654.09 & 23.71 & 8.69 & 15.12 & 0.056 & 0.321 \\
\hline 50 & 1434.92 & 25.12 & 9.825 & 16.63 & 0.060 & 0.272 \\
\hline 60 & 1343.75 & 25.26 & 10.96 & 18.14 & 0.063 & 0.239 \\
\hline 70 & 1788.83 & 18.13 & 11.43 & 19.77 & 0.044 & 0.238 \\
\hline 80 & 1686.08 & 18.56 & 11.9 & 21.4 & 0.030 & 0.237 \\
\hline 100 & 1723.40 & 17.24 & 12.85 & 23.06 & 0.026 & 0.204 \\
\hline 125 & 1587.11 & 17.92 & 18.9 & 34.4 & 0.027 & 0.248 \\
\hline 150 & 1572.63 & 17.55 & 22.3 & 41.2 & 0.022 & 0.252 \\
\hline 200 & 1373.73 & 19.32 & 29.11 & 54.43 & 0.019 & 0.253 \\
\hline
\end{tabular}

Table 5 .

Diesel genset unit database.

\begin{tabular}{lcc}
\hline & Battery bank input data & \\
\hline Symbol & Description & Value \\
\hline$V_{d c b c}$ & Battery voltage [V] & 2 \\
\hline$V_{d c s i s t}$ & DC system voltage [V] & 48 \\
\hline$C_{\text {rate }}$ & Capacity rate [h] & 5 \\
\hline$\eta_{b a t \_c}$ & Charge efficiency & 0.9 \\
\hline$\eta_{b a t \_d}$ & Discharge efficiency & 1 \\
\hline$\sigma$ & Self-discharge rate & 0.000083 \\
\hline$L_{b a t}$ & Lifecycle [years] & 10 \\
\hline$\delta_{b a t}$ & Factor of the initial capital cost invested for the battery bank & 0.7 \\
\hline$\rho_{b a t}$ & Fixed OM factor as ratio of the battery bank initial investment & 0.02 \\
\hline$D O D_{\text {max }}$ & Maximum depth of discharge & 0.5 \\
\hline
\end{tabular}

Table 6.

Battery bank technical inputs. 
Methodology for Sizing Hybrid Battery-Backed Power Generation Systems in Off-Grid Areas DOI: $h t t p: / / d x$.doi.org/10.5772/intechopen. 88830

\subsubsection{Battery bank technical data}

In this chapter book, vented lead-acid battery banks only are considered. This kind of battery cells are often selected for large energy storage banks due the low cost, low maintenance, and high cycle stability. Table 6 shows the input data required by the battery bank. The battery bank charge and discharge efficiency and the self-discharge ratio is taken from [24]. The maximum depth of discharge is set in 0.5 since the battery bank can accomplish 3000 cycles during its life service

\begin{tabular}{|c|c|c|c|c|c|}
\hline $\begin{array}{l}\text { Battery cell } \\
\text { capacity }[\mathrm{Ah}] \text { at } \\
\text { C10 }\end{array}$ & $\begin{array}{c}\text { Battery cell } \\
\text { capacity }[\mathrm{kWh}] \\
\text { at } \mathrm{C10}\end{array}$ & $\begin{array}{l}\text { Battery cell } \\
\text { voltage [V] }\end{array}$ & $\begin{array}{l}\text { \# of cycles } \\
\text { at } 50 \% \\
\text { DOD }\end{array}$ & $\begin{array}{c}\text { Price per } \\
\text { unit [USD] } \\
(€)\end{array}$ & $\begin{array}{c}\text { Price per kWh } \\
{[\mathrm{USD} / \mathrm{kWh}](€)}\end{array}$ \\
\hline 280 & 0.56 & 2 & 3000 & 114.00 & 203.57 \\
\hline 350 & 0.7 & 2 & 3000 & 135.00 & 192.86 \\
\hline 420 & 0.84 & 2 & 3000 & 153.00 & 182.14 \\
\hline 520 & 1.04 & 2 & 3000 & 161.00 & 154.81 \\
\hline 620 & 1.24 & 2 & 3000 & 186.00 & 150.00 \\
\hline 730 & 1.46 & 2 & 3000 & 210.00 & 143.84 \\
\hline 910 & 1.82 & 2 & 3000 & 234.00 & 128.57 \\
\hline 1070 & 2.14 & 2 & 3000 & 303.00 & 141.59 \\
\hline 1220 & 2.44 & 2 & 3000 & 330.00 & 135.25 \\
\hline 1370 & 2.74 & 2 & 3000 & 361.00 & 131.75 \\
\hline 1520 & 3.04 & 2 & 3000 & 389.00 & 127.96 \\
\hline 1670 & 3.34 & 2 & 3000 & 426.00 & 127.54 \\
\hline 1820 & 3.64 & 2 & 3000 & 460.00 & 126.37 \\
\hline 2170 & 4.34 & 2 & 3000 & 538.00 & 123.96 \\
\hline 2540 & 5.08 & 2 & 3000 & 664.00 & 130.71 \\
\hline 2900 & 5.8 & 2 & 3000 & 744.00 & 128.28 \\
\hline 3250 & 6.5 & 2 & 3000 & 834.00 & 128.31 \\
\hline 3610 & 7.22 & 2 & 3000 & 906.00 & 125.48 \\
\hline 3980 & 7.96 & 2 & 3000 & 981.00 & 123.24 \\
\hline 4340 & 8.68 & 2 & 3000 & 1056.00 & 121.66 \\
\hline 4700 & 9.4 & 2 & 3000 & 1097.00 & 116.70 \\
\hline
\end{tabular}

Table 7.

Battery cell database.

\begin{tabular}{lcc}
\hline & System inputs parameters & \\
\hline Symbol & Description & Value \\
\hline$R$ & Time of the project [years] & 20 \\
\hline$i_{r}$ & Real interest rate $[\%]$ & 8.08 \\
\hline$c_{\text {loss }}$ & Cost of energy loss [USD $/ \mathrm{kWh}]$ & 0.2 \\
\hline$\Delta$ & Fiscal incentive factor & 0.9038 \\
\hline
\end{tabular}

Table 8.

System input parameters. 


\begin{tabular}{lcc}
\hline & PSO input parameters & \\
\hline Symbol & Description & Value \\
\hline$N_{P V l}$ & Lower bound number of PV modules & 0 \\
\hline$w_{D G l}$ & Lower bound nominal power of diesel & 0 \\
\hline$N_{B_{p l}}$ & Lower bound number of battery cell in parallel & 0 \\
\hline$E_{b c e l l, \text { nom } l}$ & Lower bound nominal capacity of battery cell $[\mathrm{kWh}]$ & 0 \\
\hline$N_{P V u}$ & Upper bound number of PV modules & 20,000 \\
\hline$w_{D G u}$ & Upper bound nominal power of diesel unit in $[\mathrm{kW}]$ & 200 \\
\hline$N_{B_{p} u}$ & Upper bound number of battery cell in parallel & 10 \\
\hline$E_{b c e l l, \text { nom }}$ & Mpper bound nominal capacity of battery cell $[\mathrm{kWh}]$ & 9.40 \\
\hline Max & Maximum number of iterations & 50 \\
\hline$n P o p$ & Population size & 200 \\
\hline$w$ & Inertia coefficient max & 1 \\
\hline$w_{\text {max }}$ & Inertia coefficient min & 1.5 \\
\hline$w_{\text {min }}$ & Social acceleration coefficient & 0.9 \\
\hline$c_{1}$ & Inertia coefficient & 0.5 \\
\hline$c_{2}$ & Inal acceleration coefficient \\
\hline
\end{tabular}

Table 9.

PSO input parameters.

according the datasheet. Other values as maintenance cost, $\rho_{b a t}$, and the fraction of reposition cost, $\delta_{b a t}$, are set according to the recommendation of experts in the energy sector.

The main characteristics and price of the battery cells of the reference used in this work are presented in Table 7. The information was obtained from inquiries to local companies.

\subsubsection{System inputs}

The system input parameters are shown in Table 8. The cost of energy lost is assumed in $0.2 \mathrm{USD} / \mathrm{kWh}$. This value depends on the necessities and characteristics of the users of the select location. The interest rate considered in this work is $8.08 \%$ taken in [25].

Fiscal incentive factor is calculated applying an effective corporate tax income rate of $33 \%$. The resulting incentive factor is 0.938 .

The parameters for the PSO algorithm and the boundaries for each decision variable are shown in Table 9.

\section{Results of the case study}

Table 10 summarized the obtained results after applying the proposed sizing methodology. The best cost achieved was $0.2090 \mathrm{USD} / \mathrm{kWh}$ being the lowest obtained. The optimization results deliver no only the design (number of components) but also economic and reliability indicators. 
Methodology for Sizing Hybrid Battery-Backed Power Generation Systems in Off-Grid Areas DOI: http://dx.doi.org/10.5772/intechopen.88830

\begin{tabular}{lccccc}
\hline Component & Design & Unit & Indicator & Value & Unit \\
\hline$N_{p v}$ & 13 & Units & $C C_{p v}$ & 7800.00 & USD \\
\hline$P_{p v_{s t c}}$ & 3.9 & {$[\mathrm{kWp}]$} & $C C_{D G}$ & 48257.99 & USD \\
\hline$w_{D G}$ & 25 & {$[\mathrm{~kW}]$} & $C C_{b a t}$ & 3864.00 & USD \\
\hline$N_{D G}$ & 2 & Units & $O \& M_{p v}$ & 78.00 & USD/year \\
\hline$P_{D G}$ & 50 & {$[\mathrm{~kW}]$} & $O \& M_{D G_{f}}$ & 4825.80 & USD/year \\
\hline$N_{b p}$ & 1 & Units & $O \& M_{D G_{v}}$ & 26884.74 & USD/year \\
\hline$N_{b s}$ & 24 & Units & $O \& M_{D G}$ & 31710.54 & USD/year \\
\hline$N_{b a t}$ & 24 & Units & $O \& M_{b a t}$ & 77.28 & USD/year \\
\hline$E_{b c e l l, n o m}$ & 1.04 & {$[\mathrm{kWh}]$} & $R C_{D G}$ & 7019.48 & USD \\
\hline$E_{b a t, n}$ & 24.96 & {$[\mathrm{kWh}]$} & $R C_{b a t}$ & 1243.60 & USD \\
\hline & & $F C$ & 38406.77 & {$[1]$} \\
\hline & & $A C S_{a d j}$ & 38737.05 & USD/year \\
\hline & & $L P S P$ & 1.25 & $\%$ \\
\hline & & $C O E_{a d j}$ & 0.26 & USD/kWh \\
\hline & & $A C_{l o s s}$ & 475.03 & USD/year \\
\hline & & $L P G$ & 0.00 & 0.21 & USD/kWh \\
\hline
\end{tabular}

Table 10.

Results of the case study.

\section{Conclusions}

In this work, an optimization methodology was developed and described in detail to help sizing HRSE integrated by photovoltaic and diesel generation with energy storage.

The main features of the sizing methodology developed were as follows: (a) it allows the simulation of hybrid renewable systems and the evaluation of its economic and reliability integrated by diesel and photovoltaic generation with energy storage, (b) the dispatch strategy developed prioritize the use of renewable energy among other energy sources, and (c) fiscal incentives granted by the Act 1715 of 2014 in Colombia were considered on the calculation of the cost of energy using the fiscal incentive factor.

The reliability of the system was included in the objective function of the PSO algorithm through the annual cost of the energy not supplied. Also a fiscal incentive factor was used to include the financial benefits granted by the Act 1715 of 2014 in Colombia to non-conventional renewable source of energy. The results were obtained after simulating the energy flow of the system for 1 year with 1-hour resolution.

Dispatch strategy was described in detail, prioritizing the use of renewable resource over diesel generation to supply the load. Also diesel generation cannot be used to charge the battery bank. This condition was based on the fact that, in offgrid areas, the complications associated to supply the fuel and the maintenance of DG units are commonly underestimated. 
It is expected that this work will help the process of designing HRES in noninterconnected areas, thus contributing to the development of these locations and improving the life quality of the population living on these places.

\section{Acknowledgements}

The authors gratefully acknowledge the financial support provided by the Colombia Scientific Program within the framework called "Ecosistema Científico" (Contract No. FP44842-218-2018).

\section{Author details}

Oswaldo A. Arraez-Cancelliere, Nicolás Muñoz-Galeano* and Jesús M. López-Lezama

Facultad de Ingeniería, Grupo GIMEL, Universidad de Antioquia, Medellín, Colombia

*Address all correspondence to: nicolas.munoz@udea.edu.co

\section{IntechOpen}

(C) 2019 The Author(s). Licensee IntechOpen. This chapter is distributed under the terms of the Creative Commons Attribution License (http://creativecommons.org/licenses/ by/3.0), which permits unrestricted use, distribution, and reproduction in any medium, provided the original work is properly cited. (cc) BY 


\section{References}

[1] Salas V, Suponthana W, Salas RA. Overview of the off-grid photovoltaic diesel batteries systems with AC loads. Applied Energy. 2015;157:195-216. DOI: 10.1016/j.apenergy.2015.07.073

[2] Shaahid SM, Elhadidy A. Opportunities for utilization of standalone hybrid (photovoltaic + diesel + battery) power systems in hot climates. Renewable Energy. 2003;28: 1741-1753. DOI: $10.1016 /$ S0960-1481 (03) 00013-2

[3] Shaahid SM, El-Amin I. Technoeconomic evaluation of off-grid hybrid photovoltaic-diesel-battery power systems for rural electrification in Saudi Arabia-A way forward for sustainable development. Renewable Energy. 2009; 13:625-633. DOI: 10.1016/j.rser.2007. 11.017

[4] Tsuanyo D, Azoumah Y, Aussel D, Neveu P. Modeling and optimization of batteryless hybrid PV (photovoltaic)/ diesel systems for off-grid applications. Energy. 2015;86:152-163. DOI: 10.1016/ j.energy.2015.03.128

[5] Jeyaprabha SB, Selvakumar AI. Optimal sizing of photovoltaic/battery/ diesel based hybrid system and optimal tilting of solar array using the artificial intelligence for remote houses in India. Energy and Buildings. 2015;96: 40-52. DOI: 10.1016/j.enbuild.2015. 03.012

[6] Belmili H, Haddadi M, Bacha S, Almi MF, Bendib B. Sizing stand-alone photovoltaic-wind hybrid system: Techno-economic analysis and optimization. Energy Review. 2014;30: 821-832. DOI: 10.1016/j.rser.2013.11.011

[7] Luna-Rubio R, Trejo-Perea M, Vargas-Vázquez D, Ríos-Moreno GJ. Optimal sizing of renewable hybrids energy systems: A review of methodologies. Solar Energy. 2012;
86(4):1077-1088. DOI: 10.1016/j. solener.2011.10.016

[8] Erdinc O, Uzunoglu M. Optimum design of hybrid renewable energy systems: Overview of different approaches. Renewable and Sustainable Energy Reviews. 2012;16(3):1412-1425. DOI: 10.1016/j.rser.2011.11.011

[9] Ahmad Khan A, Iqbal M, Qaisar S, Anpalagan A. A compendium of optimization objectives, constraints, tools and algorithms for energy management in microgrids. Renewable and Sustainable Energy Reviews. 2016; 58:1664-1683. DOI: 10.1016/j.rser.2015. 12.259

[10] Siddaiah R, Saini P. A review on planning, configurations, modeling and optimization techniques of hybrid renewable energy systems for off grid applications. Renewable and Sustainable Energy Reviews. 2016;58:376-396. DOI: 10.1016/j.rser.2015.12.281

[11] Prakash P, Khatod DK. Optimal sizing and siting techniques for distributed generation in distribution systems: A review. Renewable and Sustainable Energy Reviews. 2016;57: 111-130. DOI: 10.1016/j.rser.2015.12.099

[12] Mandelli S, Brivio C, Colombo E, Merlo M. A sizing methodology based on levelized cost of supplied and lost energy for off-grid rural electrification systems. Renewable Energy. 2016;89: 475-488. DOI: 10.1016/j.renene. 2015.12.032

[13] Haghighat Mamaghani A, Avella Escandon SA, Najafi B, Shirazi A, Rinaldi F. Techno-economic feasibility of photovoltaic, wind, diesel and hybrid electrification systems for off-grid rural electrification in Colombia. Renewable Energy. 2016;97: 293-305. DOI: 10.1016/j.renene.2016. 05.086 
[14] Hatata AY, Osman G, Aladl MM. An optimization method for sizing a solar/ wind/battery hybrid power system based on the artificial immune system. Sustainable Energy Technologies and Assessments. 2018;27:83-93. DOI: 10.1016/j.seta.2018.03.002

[15] Mohammed A, Pasupuleti J, Khatib T, Elmenreich W. A review of process and operational system control of hybrid photovoltaic/diesel generator systems. Renewable and Sustainable Energy Reviews. 2015;44:436-446. DOI: 10.1016/j.rser.2014.12.035

[16] Ameen AM, Pasupuleti J, Khatib T. Simplified performance models of photovoltaic/diesel generator/battery system considering typical control strategies. Energy Conversion and Management. 2015;99:313-325

[17] Kashefi Kaviani A, Riahy GH, Kouhsari SM. Optimal design of a reliable hydrogen-based stand-alone wind/PV generating system, considering component outages. Renewable Energy. 2009;34(11): 2380-2390. DOI: $10.1016 /$ j. renene.2009.03.020

[18] Fathima AH, Palanisamy P. Optimization in microgrids with hybrid energy systems-A review. Renewable and Sustainable Energy Reviews. 2015; 45:431-446. DOI: 10.1016/j.rser. 2015.01.059

[19] Chauhan A, Saini RP. A review on integrated renewable energy system based power generation for stand-alone applications: Configurations, storage options, sizing methodologies and control. Renewable and Sustainable Energy Reviews. 2014;38:99-120. DOI: 10.1016/j.rser.2014.05.079

[20] Hernández-Moro J, Martínez-Duart JM. Analytical model for solar PV and CSP electricity costs: Present LCOE values and their future evolution. Renewable and Sustainable Energy
Reviews. 2013;20:119-132. DOI:

10.1016/j.rser.2012.11.082

[21] Zhang J, Huang L, Shu J, Wang H, Ding J. Energy management of PVdiesel-battery hybrid power system for island stand-alone micro-grid. Energy Procedia. 2017;105:2201-2206. DOI: 10.1016/j.egypro.2017.03.622

[22] CNM-IPSE [Internet]. 2018.

Available from: http://www.ipse.gov.co/ [Accessed: 11 July 2019]

[23] Comisión de Regulación de Energía y Gas [Internet]. 2014. Available from: http://www.creg.gov.co/ [Accessed: 11 07 2019]

[24] Yang H, Zhou W, Lu L, Fang Z. Optimal sizing method for stand-alone hybrid solar-wind system with LPSP technology by using genetic algorithm. Solar Energy. 2008;82(4):354-367. DOI: 10.1016/j.solener.2007.08.005

[25] Castillo-Ramíre A, Mejía-Giraldo D, Molina-Castro JD. Fiscal incentives impact for RETs investments in Colombia. Energy Sources. 2017;12(9): 759-764. DOI: 10.1080/15567249.2016. 1276648 
Section 3

Sustainable Development and Impacts of Renewable Energies 



\title{
Towards Sustainable Rural Development in South Africa through Passive Solar Housing Design
}

\author{
Ochuko K. Overen, Edson L. Meyer and Golden Makaka
}

\begin{abstract}
Rural low-cost housing in South Africa is characterised by poor thermal performance, as these houses are designed with no consideration of utilising ambient weather conditions for indoor thermal comfort. Hence, a prototype low-cost energy efficiency house was built based on the principle of passive solar design to avert the energy burden faced by low-cost house dwellers. Passive solar design in this context is the strategic selecting and locating of building envelope components to utilise the ambient weather factor of a house to enhance indoor thermal comfort. The aim of this study is to analyse the thermal performance of the passive solar house. To this effect, the indoor and weather conditions of the house which include air temperature, relative humidity, and solar radiation were monitored. The thermal contribution of the windows was determined from the measured data. In summer, $49 \%$ of the whole building air temperature and approximately $85 \%$ of its corresponding relative humidity were found within the thermal comfort. Only $23 \%$ temperature and $78 \%$ relative humidity distributions of the whole building were in the thermal comfort zone in the winter season. The daily cumulative heat contribution of the clerestory windows with no shading material was higher than that of the south-facing windows by $1.08 \mathrm{kWh} / \mathrm{m}^{2} /$ windows in summer and $4.45 \mathrm{kWh} / \mathrm{m}^{2} /$ windows in winter.
\end{abstract}

Keywords: rural development, housing, passive solar design, thermal performance, solar energy

\section{Introduction}

Over the years, building design, occupants' behaviour, choice of technology usage, and manufacturing and construction processes have resulted in the increasing energy consumption as well as the release of greenhouse gases (GHG) in the building sector [1]. Globally the building sector consumes over $30 \%$ of total final energy, having increased by more than $35 \%$ since 1990 and, at the same time, accounting for $30 \%$ of $\mathrm{CO}_{2}$ gas emission. The building sector also accounts for half of the world electricity demand, with some region electricity consumption increased by $500 \%$ [2]. In the residential sector, energy is consumed for space heating, cooling, domestic activities, and lighting, among others. 
However, the use of improved thermal building envelope, bioclimatic design, and energy-efficient appliance, as well as light fittings, has seen the offset of energy demand from floor and population growth in the building sector [3]. Thus, final energy demand in the building sector only rose by 5\% between 2010 and 2017. Within the above specified period, a significant decline in space heating was observed, while improvement in space heating is not visible [4].

In South Africa, the housing shortage in most rural communities resulted in the mass construction of houses (low-cost) in the Reconstruction Development Program (RDP) in 1994. Since the inception of low-cost housing (LCH), more than 4.9 million households have been accommodated with over 2.3 million backlogs $[5,6]$. According to Klunne, LCH are designed with no consideration of thermal energy efficiency, as they cannot utilise solar energy for space heating. He further indicated that uncontrollable heat exchange between the inner and outer space of the house due to openings and cracks on the building envelope leaves the inner space extremely cold in winter [7]. In 2005, Overy also found that the quality of $\mathrm{LCH}$ is poor with $90 \%$ of newly built houses not conforming to the national norms and standards. In his report, he also eluded that corruption and the use of unqualified contractors (builders) are at the forefront of the nature of the houses [8]. However, LCH dwellers tend to bear the burden as they spend a significant amount of their income to achieve thermal comfort indoors [9]. Most households that cannot afford electrical energy resort to the use of firewood, coal, paraffin heaters, or thick clothing as alternative sources of energy for heating. This results in poor indoor air quality, cold-related illness, early child motility, respiratory diseases, etc. $[10,11]$. Needless to say, the provision of LCH is a positive approach to rural development in the country, but incorporating passive solar design will improve the welfare of occupants and energy consumed in space heating as well as cooling, creating sustainable rural development.

The ambient weather of a house possesses a significant amount of energy required to naturally heat or cool the inner space at little or no expense. At the same time, the uncomfortable thermal condition indoors is due to the uncontrollable interaction between the indoor and ambient weather factors [12]. Hence, to efficiently utilise the ambient weather energy indoors, a selective thermal exchange between the inner and outer environment is required; this process is known as passive solar or bioclimatic design [13]. A passive solar design uses heat movement such as conduction, convection, and radiation to admit and distribute heat in the inner space of a house.

On a typical sunny day, heat is transmitted through the windows due to radiation and conduction. The transmitted heat is stored and distributed by furniture and indoor air due to conduction and convection, respectively. Minimum infiltration air heat transfer through enhanced airtightness and controlled ventilation components are among the strategies of passive solar design. Conductive heat transfer through the perimeter walls of a passive solar house is also avoided as it is uncontrollable [14]. Regarding cooling, strategic locating and sizing of windows are used to achieve various airflow indoors. Windows at the windward and leeward side of the house create pressure difference indoors, resulting in a cross-ventilation $[15,16]$. Also, locating windows or vents at significant height results in another form of airflow known as stack effect. Stack effect occurs due to vertical air temperature variation indoor. Therefore, the rate of airflow increases with an increase in the height between the upper and lower windows or vents [17].

In both aspects of passive solar design mentioned above, the windows play a vital role, considering the building envelope components, whereas the sun and wind constitute the ambient weather influencing factors. The windows in a passive solar house are strategically located and sized to take advantage of the 
ambient climate condition effectively. A prototype rural LCH energy-efficient house was design and constructed in SolarWatt Park based on the principle of a passive solar house. The aim of this study is to analyse the thermal performance of the house. In the context of this study, the thermal performance of the house is analysed based on the indoor weather condition relative to the outdoor ambient weather and the windows.

\section{SolarWatt Park and the passive solar house}

SolarWatt Park is located at the University of Fort Hare, Alice, in the Eastern Cape, South Africa. Alice is classified in the temperate interior (zone 2) climate of South Africa [18]. Typical annual season of Alice is characterised by a hot summer and mild (no snow) winter, with an average dry bulb temperature of 29 and $15^{\circ} \mathrm{C}$, respectively. The east wind is predominant in summer, while the winter is dominated by the west wind. An average wind speed of $2.5 \mathrm{~m} / \mathrm{s}$ is experienced in Alice throughout the year [19]. A climatic map of South Africa [20], the Google earth map of SolarWatt Park and the passive solar house are presented in Figure 1.

The site was found suitable for the design and construction of the passive solar house due to its clear north side with no sunrays' obstacle such as tall trees, mountains and high-rise buildings. Therefore, the house was designed with its major glazing area facing north which is by the energy-efficient building design recommendation in South Africa $[18,20]$. A simulated daily sun path of the house with respect to its orientation is shown in Figure 2.

In the northern hemisphere, north-orientated housing design guarantees optimum sunray penetration in the winter season due to the low-angle sun. The penetrated sunrays, therefore, provide heating and daylighting indoors. However, the 44-cm long eaves are used to prevent overheating indoors during the summer season by blocking direct sunrays. To this effect, the two large north-facing windows (see Figure 2) distribute solar radiation to the northern floor area of the house, while the clerestory windows channel solar radiance to the southern floor area. Hence, even solar radiation distribution is achieved indoors.

Meanwhile, the clerestory windows enhance indoor passive cooling due to convectional current and various wind effects through effective operations of the windows [16].

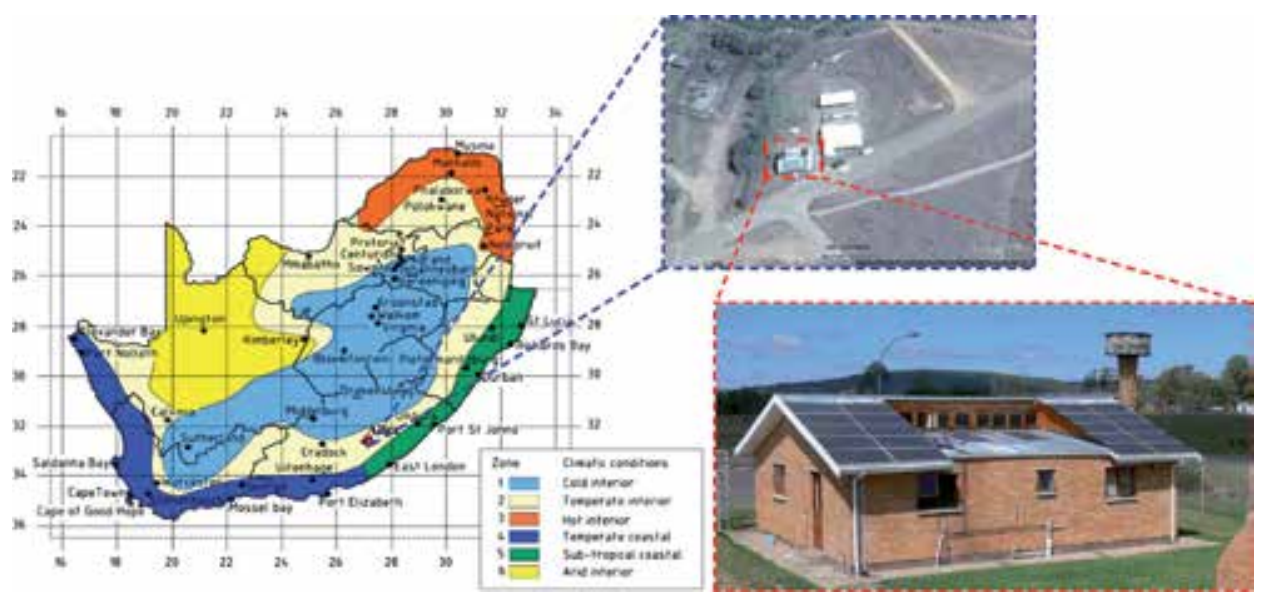

Figure 1.

Climatic map of South Africa indicating the location of the SolarWatt Park and a photo of the passive solar house. 
Furthermore, the house is made up of $10 \mathrm{~m} \times 8 \mathrm{~m}\left(80 \mathrm{~m}^{2}\right)$ floor area and consists of a bathroom, an open plan living room/kitchen and two north- and south-facing bedrooms. The floor area was arranged to ensure optimum and uniform distribution of solar radiation. The floor plan of the house indicating the floor arrangement is shown in Figure 3.

The floor plan was virtually partitioned into three thermal zones. Zone 1 marked with blue diagonal cross-hatch lines filled region is the living room/kitchen. The red diagonal up lines filled region used to indicate the north-facing bedroom is zone 2, while the south-facing bedroom is zone 3 , represented by the region filled with green vertical lines. The bathroom was not shaded since it is not considered as a thermal zone.

In 2009 , the construction of the passive solar house was estimated to be $\$ 36$, 579.55 with 1.00 USD equivalent to 11.76 ZAR, while its counterpart cost is $\$ 8505.62$ [21]. In spite of the cost margin, passive solar house presents a decent home compared to conventional low-cost house $[9,16,22]$.

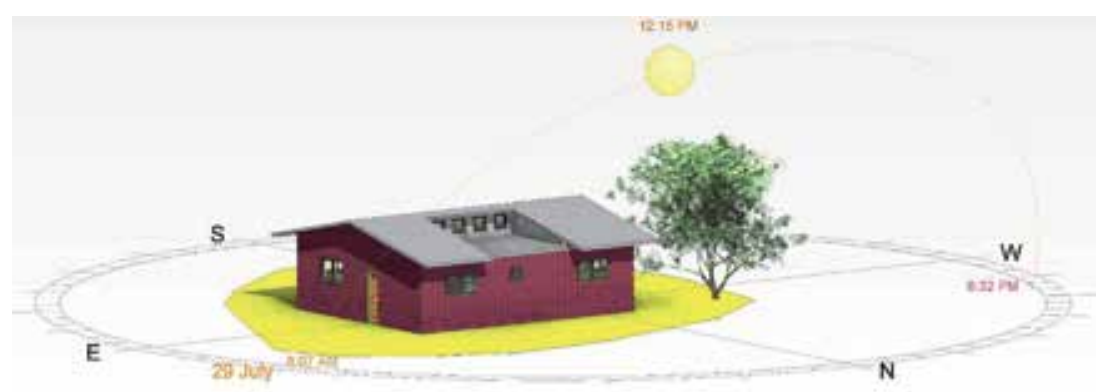

Figure 2.

A $3 D$ view sun path simulation of the house.

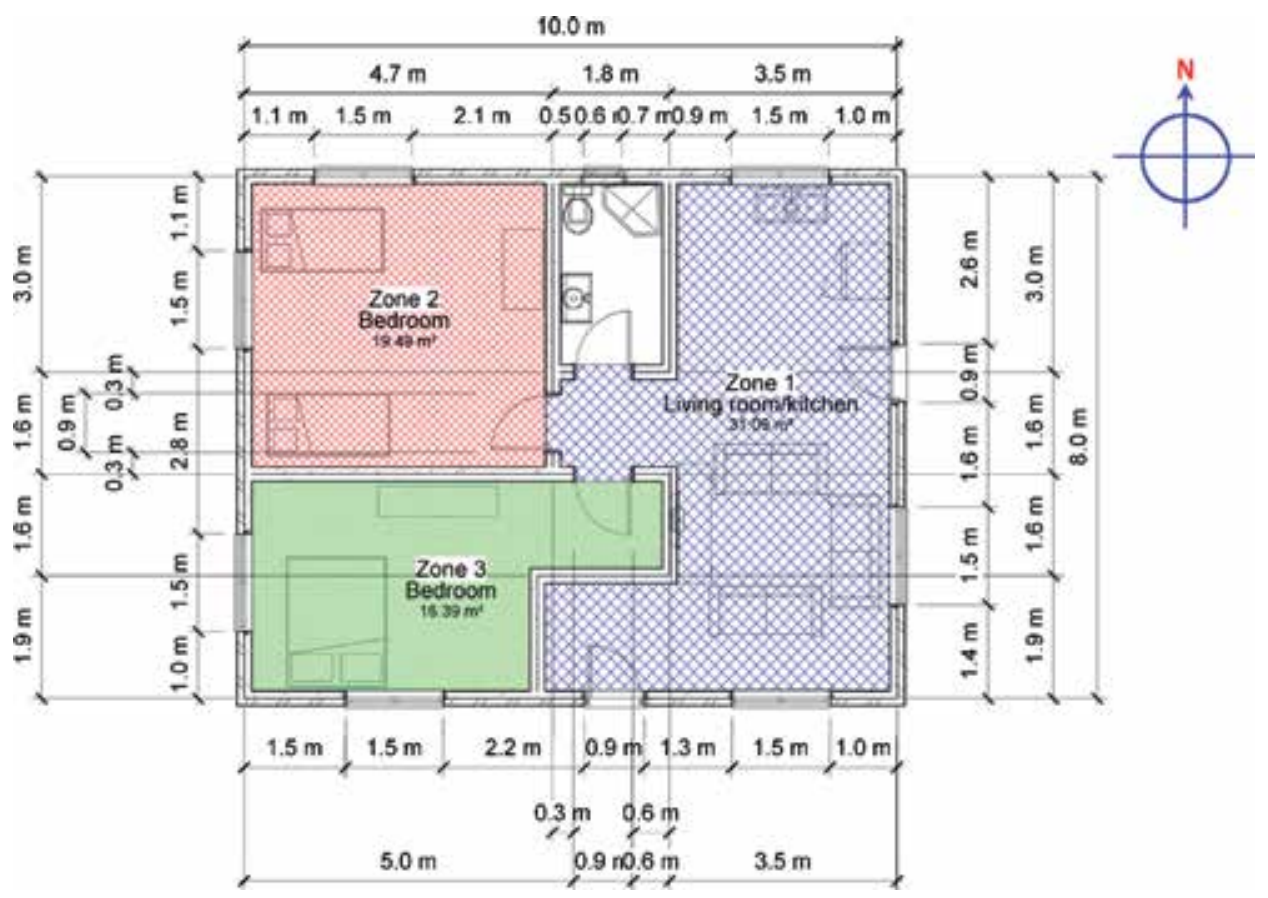

Figure 3.

The floor layout of the passive solar house indicating the floor arrangement and various zone 1. 


\section{Methods and instrumentation}

\subsection{Indoor and outdoor thermal conditions}

The indoor and outdoor thermal condition measurement deals with the air temperature and relative humidity in both environments. Therefore, HMP60 temperature and relative humidity probe were used to measure the indoor as well as the outdoor air temperature and relative humidity of all zones in the house. The HMP60 probe uses a platinum resistance temperature (PRT) detector to measure air temperature, while air relative humidity is measured by capacitive relative humidity sensor [23, 24]. The measurement specifications of HMP60 probe temperature and relative humidity sensor are given in Table 1 [25].

Three sets of HMP60 probes were used to measure the indoor air temperature and relative humidity. In each zone, one HMP60 probe was suspended at the height of $0.8 \mathrm{~m}$ to ensure that the measured air temperature is nearest to the temperature felt by the occupants. At the same time, the probe does not obstruct the activities of the occupants. The locations of the HMP60 probe in the house and a set outdoor weather station are indicated in Figure 4.

As shown in Figure 4(b), the outdoor air temperature and relative humidity measuring probe was housed in a 6-plate naturally aspirated radiation shield. The white painted radiation shield enables it to reflect solar radiation. At the same time, the louvre allows natural free flow of air through the shield, thereby keeping the probe as close as possible to the ambient air temperature (eliminating solar effect) and water vapour [26].

\subsection{Solar radiation measurements}

In this study, solar radiation measurements cover ambient global horizontal irradiance (GHI) and global irradiance at the four perimeter walls of the house. Due to atmospheric interference, the sum of direct and diffuse solar radiation reaching the earth surface, excluding albedo, is called global radiation, and it can be observed on vertical and horizontal surfaces. Thus measured global radiation on a horizontal plane is called global horizontal irradiance [27, 28].

At the right-hand side of the outdoor weather monitoring setup in Figure 4(b), the horizontally levelled Kipp and Zonen CMP-11 pyranometer was used to monitor the global horizontal irradiance (GHI). The pyranometer uses a 32-junction thermopile to measure solar radiation with a sensitivity of $8 \mu \mathrm{V} \mathrm{m}^{-2}$ and a spectral range of $285-2800 \mathrm{~nm}$. Its response time is less than $1.7 \mathrm{~s}$ (63\%) and $5 \mathrm{~s}(95 \%)$ [29]. The outdoor weather setup was elevated by $1 \mathrm{~m}$ above the roof, to ensure an unobstructed space for the radiometer. The pyranometer's dome was also cleaned twice per week to keep the dome clear of dew, dust,

\begin{tabular}{lccc}
\hline Parameters & \multicolumn{2}{c}{ Measurement range } & Accuracy $( \pm)$ \\
\hline Temperature $\left({ }^{\circ} \mathrm{C}\right)$ & -40 to +60 & & 0.6 \\
\hline Relative humidity $(\%)$ & At $0-40^{\circ} \mathrm{C}$ & $0-90$ & 3 \\
\cline { 2 - 4 } & & $90-100$ & 5 \\
\cline { 2 - 4 } & At $0-40^{\circ} \mathrm{C}$ and $+40^{\circ} \mathrm{C}$ to $+60^{\circ} \mathrm{C}$ & $0-90$ & 5 \\
\cline { 2 - 4 } & & $90-100$ & 7 \\
\hline
\end{tabular}

Table 1.

HPM60 temperature and relative humidity sensor specification. 
frost, birds' excreta, and any substance that may obstruct transmission of solar radiation.

Due to the daily sun movement, the solar irradiance at the elevations of a house varies. This, however, influences the thermal impact of the windows at various elevations. Thus, four Li-Cor 200R pyranometers with one pyranometer at each of the house elevation were used to monitor the global irradiance at the various elevations. Figure 5 shows a Li-Cor 200R pyranometer measuring the global irradiance in one of the house's elevations.

As illustrated in Figure 5, the pyranometers were mounted vertically on the outer surface of each of the perimeter wall. They were mounted at an even height of $1.8 \mathrm{~m}$. By so doing, the solar radiation falling on the walls was measured. Li-Cor pyranometer uses a silicon photovoltaic sensor mounted in a cosine-corrected head to measure solar irradiance. Together, a variable shunt resistor circuit in the cable is used to convert the measured current to a voltage signal [30].
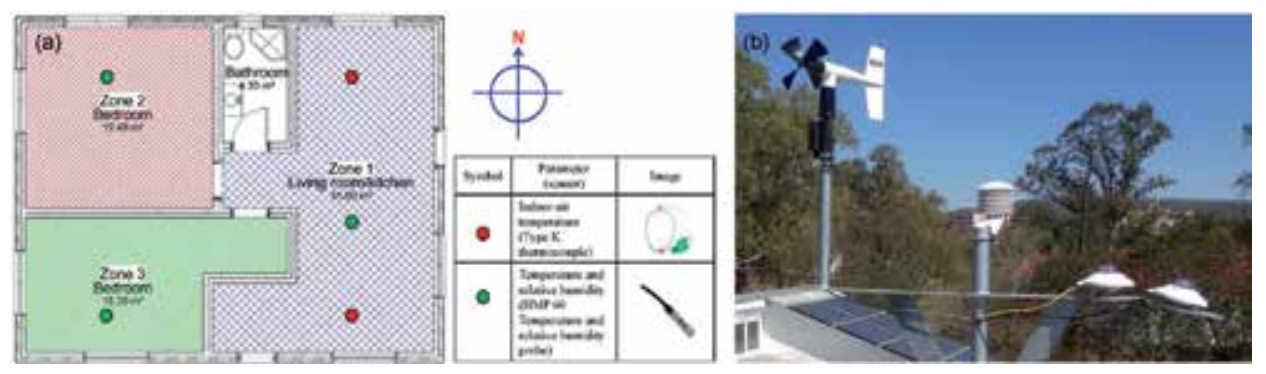

Figure 4.

(a) Floor layout of the house indicating the location of the indoor thermal sensor and (b) setup outdoor weather station.

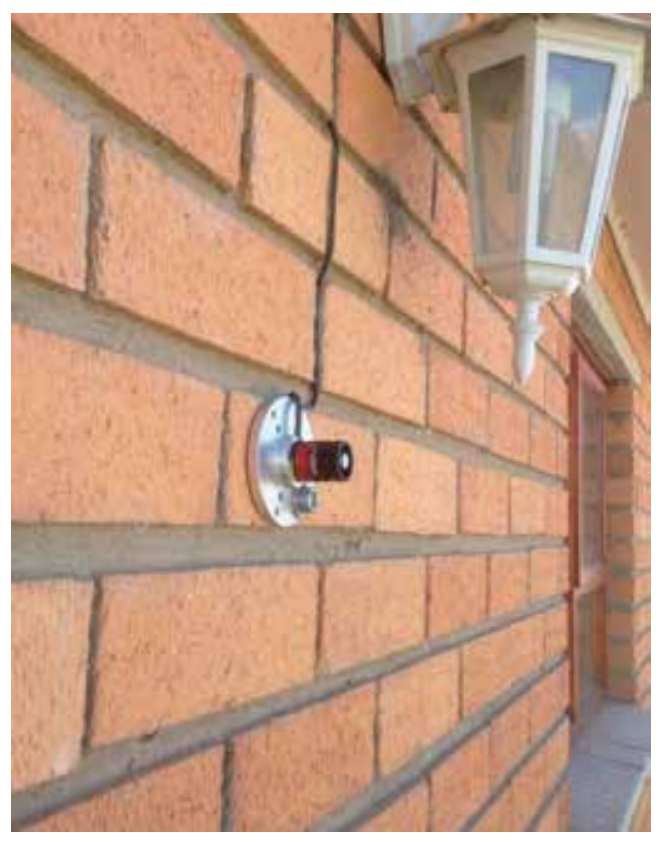

Figure 5.

Li-Cor 200R pyranometer for monitoring global vertical irradiance on the east elevation of the passive solar house. 


\section{Results and discussions}

\subsection{Ambient solar radiation analysis}

Thermal monitoring of the house which involves the global horizontal irradiance (GHI), resultant global irradiance at the various elevations, and indoor and ambient air temperature was initiated in September 2016 and continued until September 2017. Uncontrollably, 944 data entries were missed, amounting to 5\% of missing data. The missing data occurs in November 2016, December 2016, February 2017, and March 2017. The periods with missing data in the affected months were excluded in the data analysis going forward.

The measured GHI and average irradiance profile are given in Figure 6(a), while Figure 6(b) shows the monthly average GHI and total irradiation over the measurement period.

As seen in Figure 6(a), due to the measurement period considered, the winter dip, represented by June, July, and August months, was obtained at the right-hand side of the profile. This, however, did not affect the measured irradiance during the entire period. In agreement with theory $[31,32]$, the measured GHI as seen in Figure 4(a) ranges from 0 to $996.0 \mathrm{~W} / \mathrm{m}^{2}$, where periods with the sun absent produce $0 \mathrm{~W} / \mathrm{m}^{2}$ and the maximum irradiance of $996.0 \mathrm{~W} / \mathrm{m}^{2}$ was logged in February 2017 at 12 h30. Furthermore, monthly average irradiance and total irradiation were developed to portray a typical sequential distribution of annual GHI at the southern hemisphere as shown in Figure 6(b). Also, the solar irradiance and irradiation distribution were predicted using a Gaussian function. The trend of the chart tends to correspond with the solar radiation distribution in the southern hemisphere [33]. In other words, a relatively lower solar irradiance of an average of $140.5 \mathrm{~W} / \mathrm{m}^{2}$ was observed in June, July, and August, whereas the rest of the months had an average of $192.8 \mathrm{~W} / \mathrm{m}^{2}$. Due to data loss and sky formation, an irregular distribution of solar irradiance was observed in January, February, November, and December. Hence, the red and blue band areas were used to indicate the period considered as summer and winter seasons, respectively, in the thermal performance evaluation of the house.

Solar irradiance across the north, east, south, and west elevations of a house varies due to daily movement of the sun. Consequently, heat transfer through the perimeter walls varies across the elevations [34]. The global irradiance at the various elevations was measured to depict the received solar irradiance and corresponding heat transfer through the windows. Daily summer and winter average global

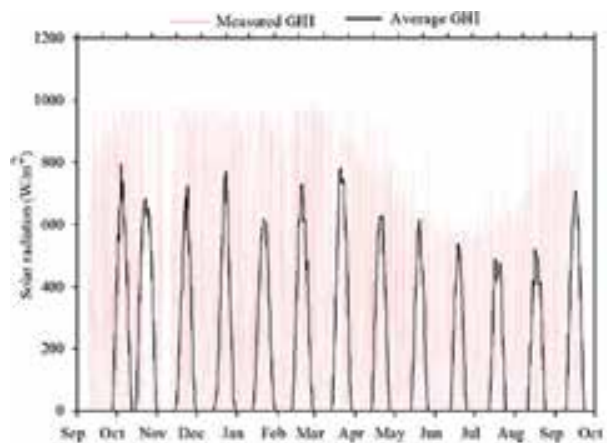

(a)

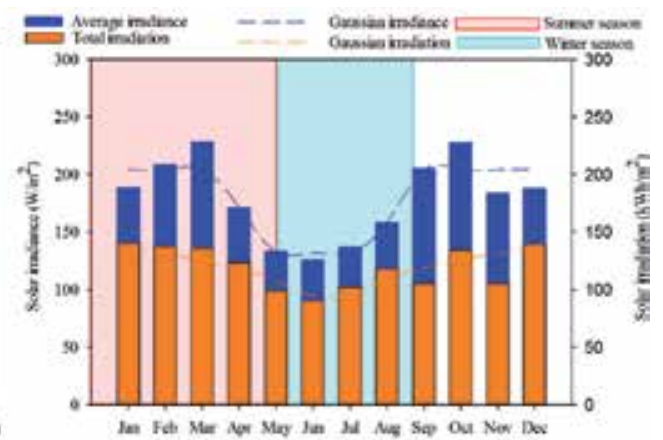

(b)

Figure 6.

(a) Measured and average global horizontal irradiance and (b) monthly average irradiance and total irradiance chart. 
irradiance at the north, east, south, and west elevations, as well as their corresponding irradiation, is given in Figure 7.

Practically, the sun travels daily from the east to the west through the north elevation in the southern hemisphere and the south elevation in the northern hemisphere $[35,36]$. The measured solar irradiance at the building elevations in Figure 7 concords with the above concept. Hence the north elevation receives a significant amount of solar radiation during the day, while the south elevation receives minimum daily solar radiation. Nevertheless, the solar irradiance at the north and south elevations peaked at approximately the same time (mid-day). Also, both irradiance (north and south elevations) followed the same trend as the GHI. The solar irradiance at the east and west elevations was also observed to peak at the early and late hours of the day, respectively.

The sharp dip in the west irradiance distribution was due to deciduous trees planted at the west side of the house. The trees were intended to shade the late afternoon sun and prevent cold winter wind. Further analysis of the solar radiation at the house elevations is given in Table 2.

As observed in Figure 7 as well as Table 1, the daily average winter irradiance at the north elevation was higher than the average GHI by $106.27 \mathrm{~W} / \mathrm{m}^{2}$. It was also observed to be higher than the north elevation average irradiance by $161.27 \mathrm{~W} /$ $\mathrm{m}^{2}$ in the summer season. The relatively high north irradiance during the winter season which is due to the low-angle winter sun is the fundamental principle of passive solar design for heating concerning the ambient weather conditions. The north elevation outperformed the others regarding daily average and maximum
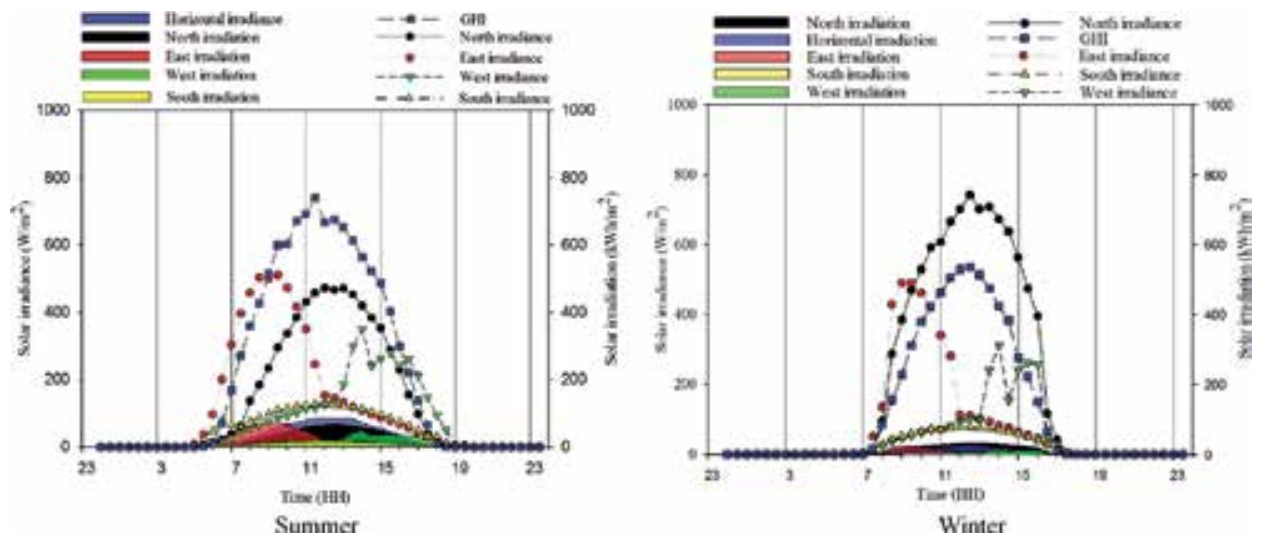

Figure 7.

Measured solar global irradiance at the elevations of the house and corresponding heat energy on typical summer and typical winter days.

\begin{tabular}{lcccccccc}
\hline $\begin{array}{l}\text { Building } \\
\text { elevation }\end{array}$ & \multicolumn{5}{c}{ Summer season } \\
\cline { 2 - 9 } & $\begin{array}{c}\text { Average } \\
\left(\mathrm{W} / \mathbf{m}^{2}\right)\end{array}$ & $\begin{array}{c}\text { Maximum } \\
\left(\mathrm{W} / \mathbf{m}^{2}\right)\end{array}$ & $\begin{array}{c}\text { Peak } \\
\text { time }\end{array}$ & $\begin{array}{c}\text { Daily } \\
\text { irradiation } \\
\left(\mathbf{k W h} / \mathbf{m}^{2}\right)\end{array}$ & $\begin{array}{c}\text { Average } \\
\left(\mathrm{W} / \mathbf{m}^{2}\right)\end{array}$ & $\begin{array}{c}\text { Maximum } \\
\left(\mathrm{W} / \mathbf{m}^{2}\right)\end{array}$ & $\begin{array}{c}\text { Peak } \\
\text { time }\end{array}$ & $\begin{array}{c}\text { Daily } \\
\text { irradiation } \\
\left(\mathbf{k W h} / \mathbf{m}^{2}\right)\end{array}$ \\
\hline North & 215.1 & 472.4 & $12 \mathrm{~h} 00$ & 3.23 & 376.4 & 741.5 & $12 \mathrm{~h} 30$ & 4.71 \\
\hline East & 188.4 & 510.7 & $09 \mathrm{~h} 30$ & 2.83 & 156.0 & 490.6 & $9 \mathrm{~h} 30$ & 1.95 \\
\hline West & 123.4 & 349.6 & $14 \mathrm{~h} 00$ & 1.85 & 92.7 & 311.7 & $14 \mathrm{~h} 00$ & 1.16 \\
\hline South & 76.6 & 126.7 & $11 \mathrm{~h} 00$ & 1.15 & 44.2 & 78.0 & $12 \mathrm{~h} 00$ & 0.55 \\
\hline
\end{tabular}

Table 2.

Typical summer day solar global radiation at the house elevations. 
irradiance as well as the daily irradiation, comparing other elevations in Table 1. The solar irradiation in Figure 7(a) and (b) serves as the instantaneous (30 min interval) heat energy received from the sun at the respective elevation, whereas, Table 1 gives the daily cumulative heat energy. Per day, the north elevation had the maximum heat energy during the winter season.

\subsection{Windows heat transfer and solar heat gain}

Heat transfer through the windows of a house is the sum of conductive and radiative heat transfer. During the day (present of the sun), both means of heat transfer coincide. While at night, conductive heat transfer is dominant. Due to the indoor and ambient air temperature difference, conductive heat transfer transpires through the windows. Conductive heat transfer through the windows is given as [37]

$$
Q_{c \theta}=\sum A U\left(T_{e o}-T_{i}\right)
$$

where $A$ is the area of the window including the frame, $U$ is the conductive heat transfer coefficient, and $T_{i}$ represents indoor air temperature. The ambient air temperature was replaced by $T_{e o}$ in Eq. (1). As indicated in Table 1, the daily irradiance varies across the house elevations; it is, however, not viable to quantify the conductive heat transfer through each window, assuming a uniform ambient air temperature. Thus, a parameter that incorporates the ambient air temperature and solar radiation was introduced to evaluate the heat transfer through the windows; this parameter is called as sol-air temperature $T_{e 0}$. Sol-air temperature also caters for convective and radiative heat transfer between the windows and ambient air film. Sol-air temperature is given by [38]:

$$
T_{e o}=T_{o}+\frac{\alpha I}{h_{o}}-\frac{\Delta q i r}{h_{o}}
$$

$h_{o}$ represents the surface (convective and radiative) heat transfer coefficient $\left(\mathrm{W} / \mathrm{m}^{2} \mathrm{~K}\right)$, while $I$ is global solar irradiance at a given elevation $\left(\mathrm{W} / \mathrm{m}^{2}\right) . \Delta q i r$ is the correction to infrared radiation transfer between a surface and the environment, if the sky temperature is different from $T_{o}\left(\mathrm{~W} / \mathrm{m}^{2}\right)$. Furthermore, $\alpha$ is the absorptance of the surface for solar radiation. Using Eq. (2) and Figure 7, the average summer and winter sol-air temperature at the various elevations of the passive solar house was computed and given in Figure 8.

In computing the sol-air temperature, longwave radiation factor $\left(\frac{\Delta q i r}{h_{\circ}}\right)$ was assumed to be $0^{\circ} \mathrm{C}$ for the windows, considering that they are vertically inclined to the sunrays. Regarding solar absorption $\alpha$ of the surfaces, a dark-coloured surface of $0.053 \mathrm{~m}^{2} \mathrm{~K} / \mathrm{W}$ was assumed [36], since the perimeter walls and the wooden frame of the windows were dark in colour. Comparing Figures 9 and 7, the sol-air temperature at each of the house elevation corresponds to their respective global irradiance. Both parameters (sol-air temperature and global irradiance) were observed to follow the same trend and peaks at the same time. However, during the absence of the sun, the sol-air temperature at the various elevations of the house was found to be approximately equal.

The sun is solely responsible for radiative heat transfer. Hence, radiative heat transfer only occurs during the period the sun is present, and it is referred to as solar heat gain. Equation (1) must be positive to achieve solar heat gain. However, the instantaneous radiative or solar heat gain through a window can be evaluated by 


$$
Q_{s o l}=A \times S C \times S H G F
$$

where $S C$ is the shading coefficient of the window. It varies with respect to the type of glazing and shading device (blind, drape, etc.). Depending on the reflection, absorption, and transmission of the glazing and shading device, a significant amount of solar heat gain is reduced or transferred through the window. An inverse function of the SC is the solar heat gain factor (SHGF). SHGF is the fraction of solar heat transmitted through a specific window. Hence, $S H G F$ takes into consideration the geographical location of the window; time of the day, month, and year; as well as orientation of the window. The $S H G F$ for windows in specific locations on the earth with respect to the parameters as mentioned above is given by ASHRAE [38]. However, a universal substitute for $S H G F$ is the solar heat gain coefficient (SHGC). The relationship between both parameters are given as

$$
S H G F=S H G C \times I
$$

Therefore Eq. (3) can be rewritten as

$$
Q_{s o l}=A \times S C \times S H G C \times I
$$

Hence, the instantaneous heat transfer through the house windows at the various elevations was computed by combining Eqs. (1) and (5) together with Figures 7 and 8 . The resultant summer and winter daily heat transfer through the house windows is given in Figure 9(a) and (b), respectively.

The following assumptions were made to obtain the profile given in Figure 10. The SHGC and U-value of a timber frame window are 0.77 and $5.6 \mathrm{~W} / \mathrm{m}^{2} \mathrm{~K}$, respectively [18]. In all windows excluding the clerestory windows, semiopen weave and medium colour single drapes were used. Hence, the SC of the windows was 0.51 . On the other hand, no drapes were used in the clerestory windows; thus the SC was 0.95 [39]. The area of the frame and thermal lag factor of the windows were ignored. In terms of operation, all windows were closed at all times.

In both figures, the north-facing windows had the maximum heat gain. This includes the clerestory and north perimeter windows. Heat gain through the clerestory windows was found to be maximum with $759 \mathrm{~W}$ at $14 \mathrm{~h} 00$ and $1356.50 \mathrm{~W}$ at $12 \mathrm{~h} 30$, in summer and winter, respectively. The daily average heat gain through the same windows was $507.72 \mathrm{~W}$ in summer and $896.51 \mathrm{~W}$ in winter. The north
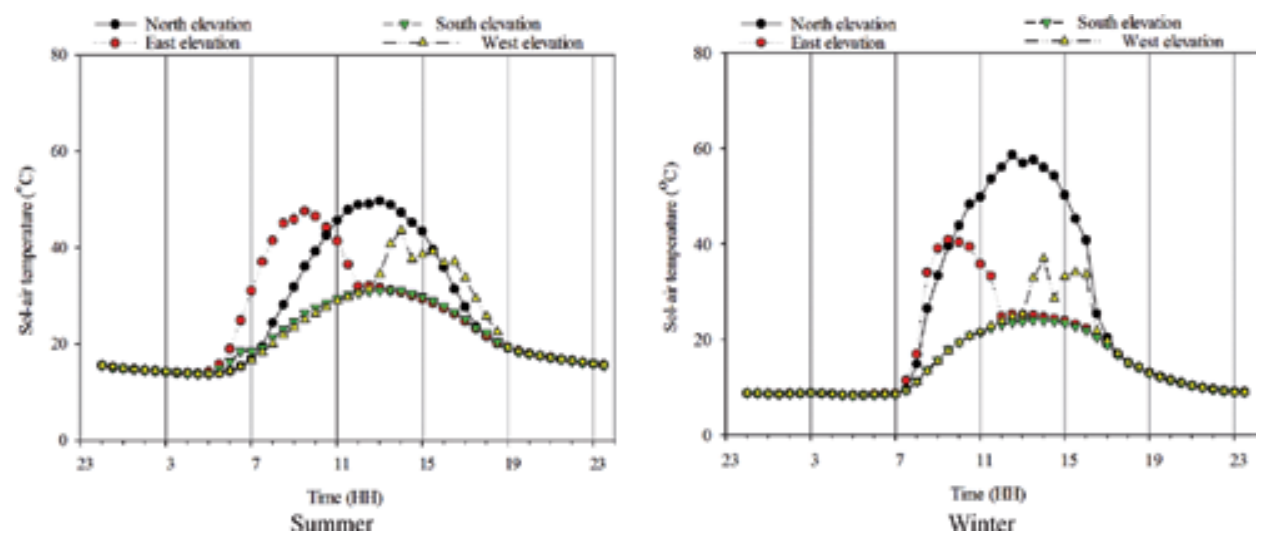

Figure 8.

Sol-air temperature at various elevations of a passive solar house on average summer and winter days. 

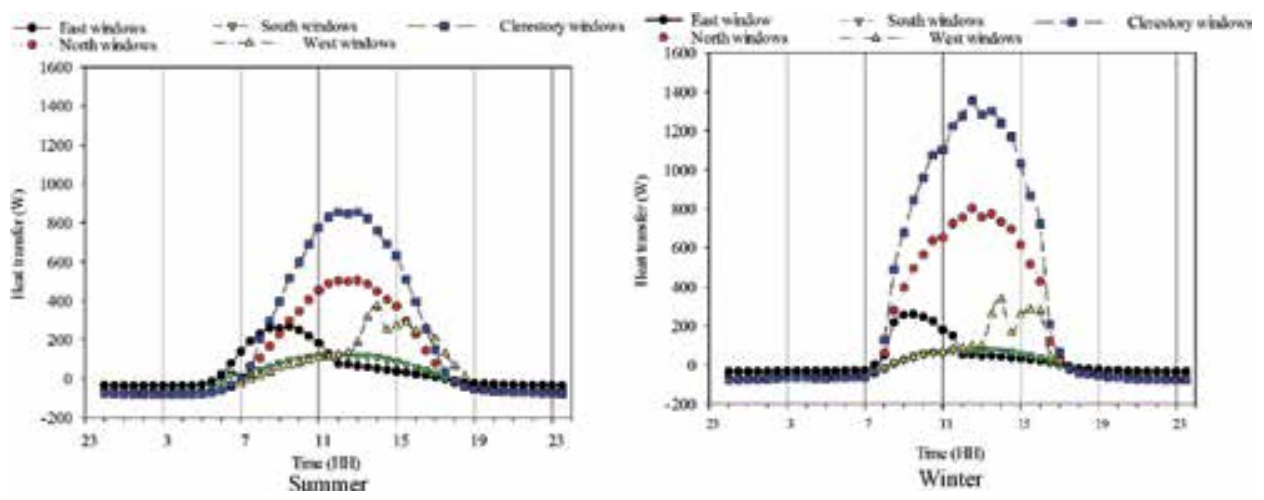

Figure 9.

Typical summer and winter daily heat transfer through the windows of a passive solar house.

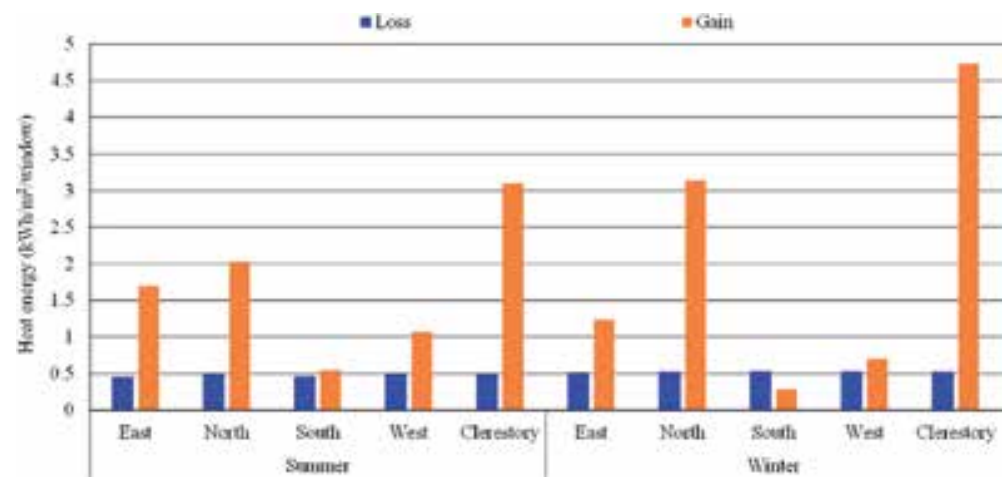

Figure 10.

Seasonal daily windows' cumulative heat energy.

perimeter windows' heat transfer follows the same trend but 79 and $41 \%$ lesser in summer and winter, respectively.

The least heat gain was obtained at the south perimeter windows. Their daily average heat gain was $76.29 \mathrm{~W}$ in summer and $50.85 \mathrm{~W}$ in winter. Heat transfer through the south windows was $85 \%$ lesser than that of the clerestory windows in summer and $94 \%$ lower in the winter season. The clerestory windows also had the maximum heat loss. The daily average heat loss difference between the clerestory windows and the other perimeter windows was 15.62 and $14.42 \mathrm{~W}$ in summer and winter, respectively. Furthermore, the daily cumulative heat energy through the windows in summer and winter is given in Figure 10.

The clerestory windows generate $3.10 \mathrm{kWh} / \mathrm{m}^{2} /$ window in summer and 4.73 $\mathrm{kWh} / \mathrm{m}^{2} /$ window in winter. This results in 9.30 and $18.60 \mathrm{kWh} / \mathrm{m}^{2}$ daily cumulative heat energy generated in the living room and south-facing bedroom in summer, as well as $14.19 \mathrm{kWh} / \mathrm{m}^{2}$ in the living room and $28.38 \mathrm{kWh} / \mathrm{m}^{2}$ in the south-facing bedroom in winter. Once again, the south perimeter windows were the most underperforming. The daily cumulative heat energy gain through the south windows was 82 and 94\% lesser than that of the clerestory windows in summer and winter, respectively. An average daily cumulative heat energy loss of $0.48 \mathrm{kWh} / \mathrm{m}^{2} /$ window was obtained in all windows in summer. Also in winter, $0.52 \mathrm{kWh} / \mathrm{m}^{2} /$ window was obtained. Besides, the east perimeter windows had the maximum heat energy loss with $0.45 \mathrm{kWh} / \mathrm{m}^{2} /$ window in summer and $0.51 \mathrm{kWh} / \mathrm{m}^{2} /$ window in winter.

The clerestory windows show significant heat contribution to the inner space of the house. From the findings, it indicates that the clerestory windows were able to 
offset the underperforming south perimeter windows. Also, irrespective of size (glass surface area), the clerestory windows outperformed the other windows in both seasons in terms of heat gain. It can be said that the performance of the windows is a factor of the orientation of the house rather than the surface area of the windows (glass). Additionally, the solar heat gain through the windows was examined. The summer and winter daily average solar irradiation on the outer surface of the house windows and the resultant heat energy gain are given in Figures 11 and 12, respectively.

From Figure 11, the south perimeter windows had minimum heat energy transmission. It was found that $48 \%$ /window of solar irradiation was transmitted through the south perimeter windows. The clerestory windows, on the other hand, had the maximum heat energy transmission with 96\%/window. Similar behaviour was observed in Figure 12. The south perimeter and clerestory windows heat energy transmission with respect to their solar irradiation were 52 and 101\%/window, respectively. Detailed findings of the solar irradiation of the house windows and the resultant heat energy transmission are given in Table 3.

As stated earlier, the heat energy transmitted through the windows (glass area) is due to simultaneous conductive and radiative heat transfer. Although, the above comparative analysis only took into consideration the radiative heat energy generated on the windows' outer surface. Also, no shading device (drapes) was used in the clerestory windows. Hence, during the winter season, more than $100 \%$ of heat energy was transmitted through the clerestory windows.

\subsection{Indoor weather conditions analysis}

The indoor air temperature and relative humidity were the focus of the indoor weather conditions analysis. Thus, both parameters of each zone in the house were measured separately to establish the thermal influence of the various activities and orientation of the rooms (see Figure 4). The seasonal daily indoor air temperature and relative humidity profiles of the different zones in the house are given in Figure 13.

In Figure 13, the vertical bar charts below and above represent the air temperature and relative humidity percentage difference, respectively, of the three zones. A minimal summer day air temperature and relative humidity percentage difference were observed. However, zone 3 had the maximum air temperature and relative
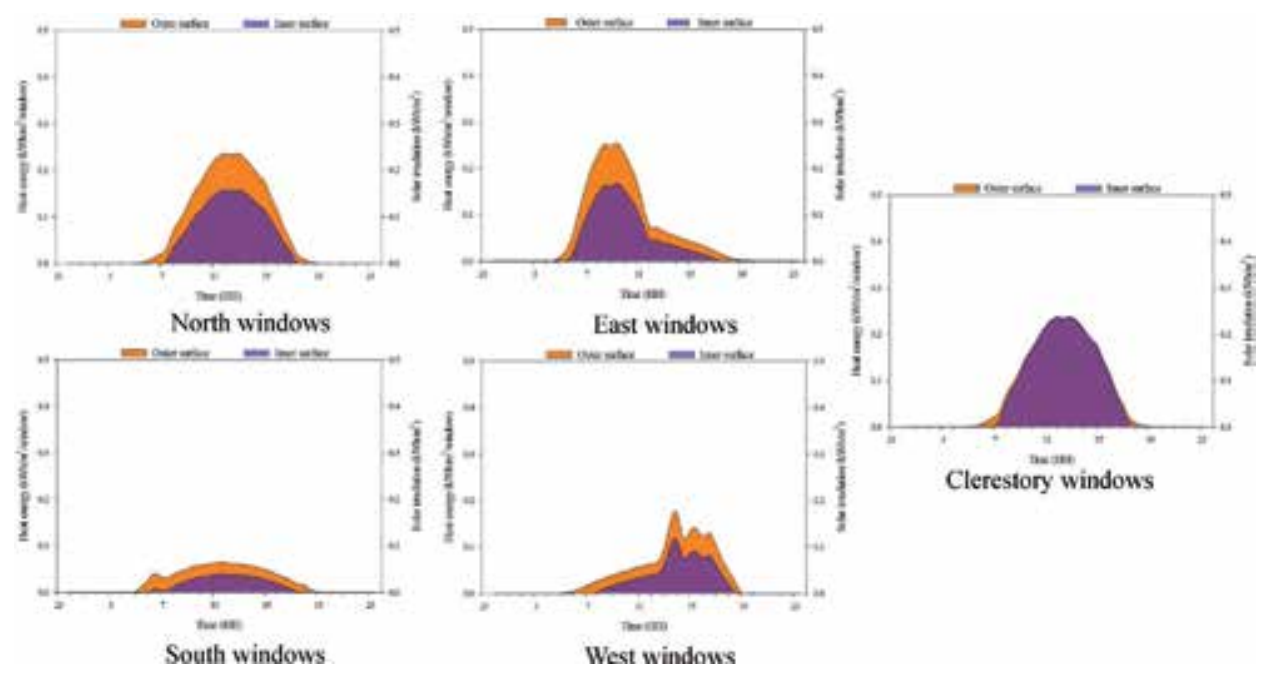

Figure 11.

Typical summer day solar heat gain of the house north, east, south, west, and clerestory windows. 
Towards Sustainable Rural Development in South Africa through Passive Solar Housing Design DOI: http://dx.doi.org/10.5772/intechopen.85997

humidity percentage difference in both days. On a typical summer day, the air temperature percentage difference was $4 \%$ at $12 \mathrm{~h} 00$ and a corresponding relative humidity of $3 \%$ at $11 \mathrm{~h} 00$. The air temperature and relative humidity percentage differences were, respectively, $16 \%$ and $12 \%$ on a typical winter day. Further findings of Figure 13 are summarised in Table 4.

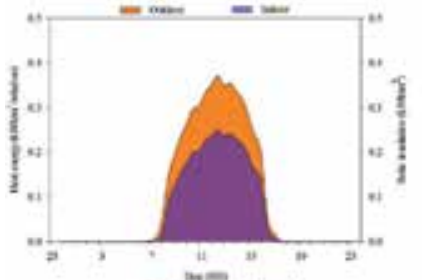

North windows

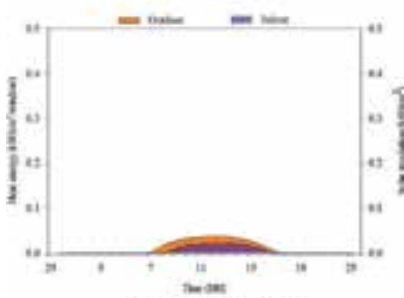

South windows

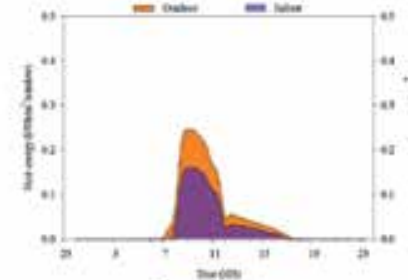

East windows

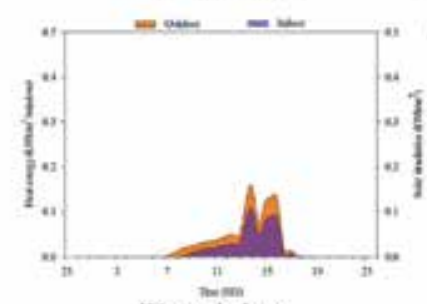

West windows

Figure 12.

Typical winter day solar heat gain of the house north, east, south, west, and clerestory windows.

\begin{tabular}{lcccc}
\hline Windows & \multicolumn{2}{c}{ Summer } & \multicolumn{2}{c}{ Winter } \\
\hline & $\begin{array}{c}\text { Solar } \\
\text { irradiation } \\
\left(\mathbf{k W h} / \mathbf{m}^{2}\right)\end{array}$ & $\begin{array}{c}\text { Transmitted heat } \\
\text { energy (\%/window) }\end{array}$ & $\begin{array}{c}\text { Solar } \\
\text { irradiation } \\
\left(\mathbf{k W h} / \mathbf{m}^{2}\right)\end{array}$ & $\begin{array}{c}\text { Transmitted heat } \\
\text { energy (\%/window) }\end{array}$ \\
\hline North & 3.23 & 63 & 4.71 & 67 \\
\hline East & 2.83 & 60 & 1.95 & 63 \\
\hline South & 1.15 & 48 & 0.55 & 52 \\
\hline West & 1.85 & 57 & 1.16 & 61 \\
\hline Clerestory & 3.23 & 96 & 4.71 & 101 \\
\hline
\end{tabular}

Table 3.

Seasonal solar heat energy transmitted through the windows.

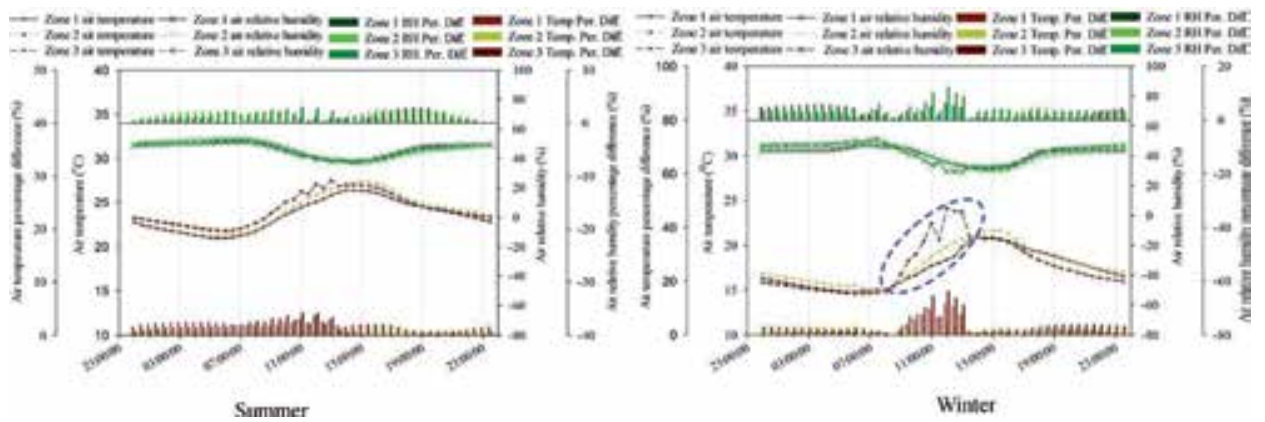

Figure 13.

Average summer and winter days' air temperature and relative humidity distribution in the various zone. 
In Tables 4 and 5, the daily swing refers to the difference between the daily maximum and minimum air temperature and relative humidity. In Table 4, a fairly constant daily air temperature swing with an average of $5.6^{\circ} \mathrm{C}$ was observed, although the relative humidity swing in each zone varies. This was expected given that the presence and activities of occupants in a room are an influencing factor of relative humidity.

Furthermore, varying air temperature and relative humidity were observed in each zone during the typical winter day. This implies that a relatively high diurnal temperature variation was experienced during the winter season. In addition, zone 3 had the maximum daily air temperature swing of $9.6^{\circ} \mathrm{C}$. This was as a result of the northfacing clerestory windows. Recall that the house was designed to optimise even air temperature indoors. Hence, the clerestory windows were installed to distribute solar radiation to the south floor area of the house. The blue dash circles in Figure 13 indicate air temperature increase in zone 3 (south-facing room) due to penetrated solar radiation, consequently increasing the day and night air temperature differences in the zone. Zone 3 air temperature tends to increase more in winter due to the low-angled sun experienced during the season, regarding the blue circled area in both figures.

However, the average indoor air temperature and relative humidity in all zones were obtained and used to illustrate the indoor air temperature and relative humidity distribution within the thermal comfort zone. The average air temperature of all zones was represented by the whole building air temperature, while whole building relative humidity served as the average relative humidity of the three zones. Summer season frequency distribution of the whole building and ambient air temperature, as well as their corresponding relative humidity, are shown in Figure 14.

\begin{tabular}{lcccccc}
\hline Zone & \multicolumn{2}{c}{$\mathbf{1}$} & \multicolumn{2}{c}{$\mathbf{2}$} & \multicolumn{3}{c}{$\mathbf{3}$} \\
\hline $\begin{array}{l}\text { Indoor weather } \\
\text { parameter }\end{array}$ & $\begin{array}{c}\text { Temp. } \\
\left({ }^{\circ} \mathrm{C}\right)\end{array}$ & $\mathbf{R H}(\%)$ & $\begin{array}{c}\text { Temp. } \\
\left({ }^{\circ} \mathrm{C}\right)\end{array}$ & $\mathbf{R H}(\%)$ & $\begin{array}{c}\text { Temp. } \\
\left({ }^{\circ} \mathrm{C}\right)\end{array}$ & $\mathbf{R H}(\%)$ \\
\hline Daily swing & 5.4 & 14.4 & 5.8 & 16.1 & 5.7 & 13.5 \\
\hline $\begin{array}{l}\text { Max. per. diff. (\%) } \\
\text { (equiv. temp.) }\end{array}$ & $\begin{array}{c}4 \\
\left(0.9^{\circ} \mathrm{C}\right)\end{array}$ & $\begin{array}{c}2.9 \\
(1.3 \%)\end{array}$ & $\begin{array}{c}2 \\
\left(0.5^{\circ} \mathrm{C}\right)\end{array}$ & $\begin{array}{c}2.7 \\
(1.2 \%)\end{array}$ & $\begin{array}{c}\left(1.1^{\circ} \mathrm{C}\right) \\
(1.3 \%)\end{array}$ \\
\hline Peak time & $12 \mathrm{~h} 00$ & $18 \mathrm{~h} 30$ & $16 \mathrm{~h} 00$ & $18 \mathrm{~h} 30$ & $11 \mathrm{~h} 00$ & $11 \mathrm{~h} 00$ \\
\hline $\begin{array}{l}\text { Average per. diff. }(\%) \\
\text { (equiv. temp.) }\end{array}$ & $\begin{array}{c}2 \\
\left(0.4^{\circ} \mathrm{C}\right)\end{array}$ & $\begin{array}{c}0.9 \\
(0.4 \%)\end{array}$ & $\begin{array}{c}1 \\
\left(0.2^{\circ} \mathrm{C}\right)\end{array}$ & $\begin{array}{c}1.7 \\
(0.8 \%)\end{array}$ & $\begin{array}{c}1 \\
\left(0.3^{\circ} \mathrm{C}\right)\end{array}$ & $\begin{array}{c}1.1 \\
(0.5 \%)\end{array}$ \\
\hline
\end{tabular}

Table 4.

Typical summer day indoor air temperature and relative humidity variation in the house zones.

\begin{tabular}{lcccccc}
\hline Zone & \multicolumn{2}{c}{$\mathbf{1}$} & \multicolumn{2}{c}{$\mathbf{2}$} & \multicolumn{3}{c}{$\mathbf{3}$} \\
\hline $\begin{array}{l}\text { Indoor weather } \\
\text { parameter }\end{array}$ & $\begin{array}{c}\text { Temp. } \\
\left({ }^{\circ} \mathrm{C}\right)\end{array}$ & $\mathbf{R H}(\%)$ & $\begin{array}{c}\text { Temp. } \\
\left({ }^{\circ} \mathrm{C}\right)\end{array}$ & $\mathbf{R H}(\%)$ & $\begin{array}{c}\text { Temp. } \\
\left({ }^{\circ} \mathrm{C}\right)\end{array}$ & $\mathbf{R H}(\%)$ \\
\hline Daily swing & 6.1 & 16.3 & 6.7 & 20.0 & 9.6 & 22.2 \\
\hline $\begin{array}{l}\text { Max. per. diff. }(\%) \\
\text { (equiv. temp.) }\end{array}$ & $\begin{array}{c}11.6 \\
\left(2.4^{\circ} \mathrm{C}\right)\end{array}$ & $\begin{array}{c}6.5 \\
(2.2 \%)\end{array}$ & $\begin{array}{c}4.8 \\
\left(0.9^{\circ} \mathrm{C}\right)\end{array}$ & $\begin{array}{c}5.7 \\
(1.9 \%)\end{array}$ & $\begin{array}{c}16.2 \\
\left(3.4^{\circ} \mathrm{C}\right)\end{array}$ & $\begin{array}{c}12.2 \\
(4.1 \%)\end{array}$ \\
\hline Peak time & $12 \mathrm{~h} 00$ & $12 \mathrm{~h} 00$ & $11 \mathrm{~h} 00$ & $12 \mathrm{~h} 00$ & $12 \mathrm{~h} 00$ & $12 \mathrm{~h} 00$ \\
\hline $\begin{array}{l}\text { Average per. diff. }(\%) \\
\text { (equiv. temp.) }\end{array}$ & $\begin{array}{c}2.2 \\
\left(0.4^{\circ} \mathrm{C}\right)\end{array}$ & $\begin{array}{c}3.1 \\
(1.3 \%)\end{array}$ & $\begin{array}{c}2.3 \\
\left(0.4^{\circ} \mathrm{C}\right)\end{array}$ & $\begin{array}{c}2.5 \\
(1.0 \%)\end{array}$ & $\begin{array}{c}3.9 \\
\left(0.7^{\circ} \mathrm{C}\right)\end{array}$ & $\begin{array}{c}3.6 \\
(1.5 \%)\end{array}$ \\
\hline
\end{tabular}

Table 5.

Typical winter day indoor air temperature and relative humidity variation in the house zones. 
A total of 12,607 data entries were used to develop the summer whole building and ambient air temperature and relative humidity distribution profile. As seen in Figure 14, the whole building and ambient air temperature distributions were divided into nine classes of $4.9^{\circ} \mathrm{C}$ width, and the air relative humidity frequency distribution curve is made of 11 classes of $9.99 \%$ width. Statistically, the whole building and ambient air temperature were not normally distributed. The whole building temperature skewness was 0.20 with a standard error (S.E) of 0.02 , whereas a skewness of 0.41 (S.E 0.02) was obtained for the ambient temperature. Although both whole building and ambient air relative humidity in Figure 14 were also not normally distributed, an opposite skewness was obtained. The whole building and ambient relative humidity had a skewness of -23.32 and -23.68 , respectively, both with a S.E of 0.02 .

This implies that the measured air temperature and relative humidity deviate away from their mean towards the positive and negative side, respectively, where the whole building and ambient mean values are indicated by the blue broken $\left(24^{\circ} \mathrm{C}\right)$ and solid $\left(19^{\circ} \mathrm{C}\right)$ lines in their respective classes. The broken red line $(47 \%)$ is used to identify the mean value of the whole building relative humidity, while the solid red line signifies the mean ambient relative humidity of $68 \%$.

Thermally in Figure 14, the solid grey line and band indicate the indoor air temperature $\left(20\right.$ and $\left.24^{\circ} \mathrm{C}\right)$ and relative humidity (30 and 60\%) comfort zones, respectively [40]. In this regard, $49 \%$ of the whole building air temperature and approximately $85 \%$ of its corresponding relative humidity were found within the thermal comfort, whereas only 21 and $28 \%$ of the ambient air temperature and relative humidity, respectively, were in the thermal comfort zone.

In the winter season, a total of 4386 data entries were used to develop the whole building thermal condition, ambient air temperature, and relative humidity distribution profile. Nonetheless, a similar behaviour of the whole building, ambient air temperature, and relative humidity were observed. Figure 15 shows the measured ambient and whole building air temperature as well as the resultant relative humidity during the winter season.

From Figure 15, the whole building temperature and relative humidity skewness were 0.22 and -0.21 , respectively, with a S.E of 0.04 . Meanwhile, a skewness of 0.22 was observed for the ambient air temperature, while the relative humidity skewness was -0.15 , both with a S.E of 0.04 . Hence, the whole building, ambient air temperature, and relative humidity curves are asymmetric. In other words, the mean of the whole building air temperature drifts away from the thermal comfort zone, leaving only $23 \%$ of the whole building temperature distribution in the
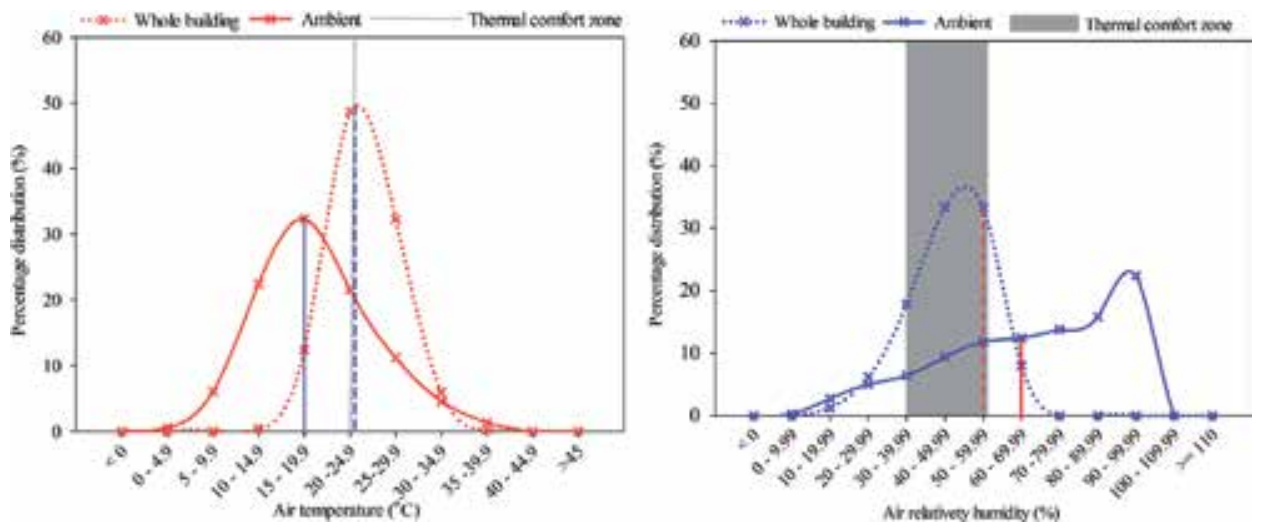

Figure 14.

Whole building and ambient air temperature and relative humidity summer season profile. 

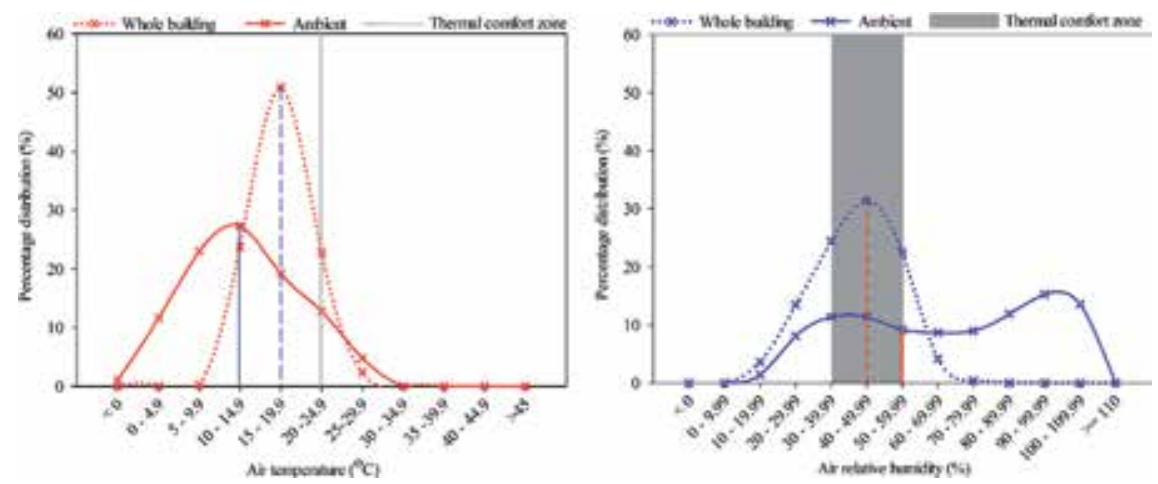

Figure 15.

Whole building and ambient air temperature and relative humidity winter season profile.

thermal comfort zone. However, the percentage of the ambient air temperature in the thermal comfort zone deviates by $10 \%$, whereas approximately 78 and $29 \%$ of the whole building and ambient air relative humidity, respectively, were inside the thermal comfort zone.

Based on the findings, it could be said that the whole building air temperature to a certain degree is influenced by the ambient air temperature given that both distributions follow the same trend in both seasons. Nevertheless, the same cannot be said for the whole building and ambient air relative humidity. In both seasons, the whole building relative humidity distribution tends to follow the whole building air temperature.

Theoretically, relative humidity is a measure in percentage of the amount of water vapour in the air compared to the amount of water vapour the air can hold at a given temperature. Considering that the amount of water vapour the air can hold mainly depends on the air temperature, an increase in air temperature increases the capacity of water vapour the air can hold. At a fixed amount of water vapour, an increase in air temperature results in a decrease of the air relative humidity and vice versa. Therefore, the measured air temperature and relative humidity in Figures 14 and $\mathbf{1 5}$ are in line with theory.

\section{Conclusion}

The aim of this study is to analyse the thermal performance of a prototype low-cost energy-efficient house in South Africa. A passive solar house in SolarWatt Park, Alice, was used in the study. The indoor and ambient weather conditions of the house were monitored. Indoor and outdoor air temperature, relative humidity, as well as global horizontal irradiance and global irradiance at the various elevations of the house constitute the weather conditions.

It was found that strategic locating of the windows provides significant daylighting and heating for the inner space of the house. Also, the heat contribution of the windows was found to be dependent on the house orientation and shading materials (blind and drape). The performance of the north-facing clerestory and south-facing windows supports this claim. The daily cumulative heat contribution of the clerestory windows with no shading material was higher than that of the south-facing windows by $1.08 \mathrm{kWh} / \mathrm{m}^{2} /$ windows in summer and $4.45 \mathrm{kWh} / \mathrm{m}^{2}$ / windows in winter. Due to conductive and radiative heat transfer which co-occurs in the windows, the clerestory windows were found to transmit more than $100 \%$ of the solar radiative energy generated on the outer surface in winter. The performance 
of the clerestory windows as shown in the findings made it an essential component of direct solar heat gain strategy in passive solar design.

It was also observed that the generated heat from the windows does not constitute overheating indoor. In summer, $49 \%$ of the whole building air temperature and approximately $85 \%$ of its corresponding relative humidity were found within the thermal comfort. Only $23 \%$ temperature and $78 \%$ relative humidity distributions of the whole building were in the thermal comfort zone in the winter season.

\section{Acknowledgements}

This work was based on the research supported in part by the National Research Foundation of South Africa (Grant number 116763). We also acknowledge the Department of Science and Technology and Govan Mbeki Research and Development Centre for supporting this research.

\section{Conflict of interest}

The authors declared no conflicts of interest regarding the authorship of this publication.

\section{Author details}

Ochuko K. Overen*, Edson L. Meyer and Golden Makaka

University of Fort Hare, Fort Hare Institute of Technology, Alice, South Africa

*Address all correspondence to: ooveren@ufh.ac.za

\section{IntechOpen}

(C) 2019 The Author(s). Licensee IntechOpen. This chapter is distributed under the terms of the Creative Commons Attribution License (http://creativecommons.org/licenses/ by/3.0), which permits unrestricted use, distribution, and reproduction in any medium, provided the original work is properly cited. (cc) BY 


\section{References}

[1] Blok K, Geng L, Harvey D, Lang S, Levermore G, Mehlwana M, et al. Residential and commercial buildings. In: Clim. Chang. 2007 Work. Gr. III Mitig. Clim. Chang. New York: Cambridge; 2007. pp. 387-446

[2] International Energy Agency. Energy Technology Perspectives 2016: Towards Sustainable Urban Energy Systems. Paris: International Energy Agency; 2016

[3] International Energy Agency and the United Nations Environment Programme. 2018 Global Status Report: Towards a zero-emission, efficient and resilient buildings and construction sector. Germany: United Nation Environment; 2018

[4] IEA. Energy Efficiency 2018Analysis and outlooks to 2040. Paris: International Energy Agency; 2018

[5] Msindo BYE. Housing backlog Protests and the demand for Housing in South Africa. Johannesburg: Public Service Accountability Monitor; 2017

[6] Parliamentary Monitoring Group. Department of Human Settlements 2016/17 Annual Report, with AuditorGeneral \&amp; DPME input | PMG [Online] . 2017. Available from: https:// pmg.org.za/committee-meeting/25122/ [Accessed: 2019-02-9]

[7] Klunne WE. Energy efficient housing to benefit South African households. Boiling Point. 2002;48:27-29

[8] Overy N. The Housing Crisis in the Eastern Cape. Johannesburg: Public Service Accountability Monitor; 2005

[9] Overen OK, Meyer EL, Makaka G. Thermal, economic and environmental analysis of a lowcost house in Alice, South Africa. Sustainability. 2017;9:425
[10] Dear KBG, McMichael AJ. The health impacts of cold homes and fuel poverty. BMJ. 2011;342:d2807. DOI: 10.1136/bmj.d2807

[11] Sustainable Energy Africa. Tackling Urban Energy Poverty in South Africa. Cape Town: Heinrich Böll Stiftung Southern Africa; 2014

[12] Eltaweel A, SU Y. Parametric design and daylighting: A literature review. Renewable and Sustainable Energy Reviews. 2017;73:1086-1103. DOI: 10.1016/j.rser.2017.02.011

[13] Victoria J, Akhtar S, Akmal W, Wan Z. Bioclimatic design approach in Dayak traditional longhouse. Procedia Engineering. 2017;180:562-570. DOI: 10.1016/j.proeng.2017.04.215

[14] Schnieders J, Feist W, Rongen L. Passive Houses for different climate zones. Energy and Buildings. 2015;105:71-87. DOI: 10.1016/j. enbuild.2015.07.032

[15] Karava P, Stathopoulos T, Athienitis AK. Airflow assessment in cross-ventilated buildings with operable façade elements. Building and Environment. 2011;46:266-279. DOI: 10.1016/j.buildenv.2010.07.022

[16] Overen OK, Meyer EL, Makaka G, Ziuku S, Mamphweli S. Zonal air exchange rate of a passive solar house and resultant sensible air heat transfer. Indoor and Built Environment. 2018;0:1-13. DOI: 10.1177/1420326X18804605

[17] Jo JH, Lim JH, Song SY, Yeo MS, Kim KW. Characteristics of pressure distribution and solution to the problems caused by stack effect in high-rise residential buildings. Building and Environment. 2007;42:263-277. DOI: 10.1016/j. buildenv.2005.07.002 
[18] South African Bureau of Standards. SANS 204: Energy efficiency in buildings; 2011; South Africa. Pretoria: South African Bureau of Standards; 2011. pp. 1-65

[19] Conradie DCU. South Africa's climatic zones: Today, tomorrow. In: International Green Building Conference and Exhibition. Pretoria: Center of Science and Industrial Research; 2012;2012. pp. 1-9

[20] South African Bureau of Standards, SANS 10400-XA. The application of National Building Regulations, Part X: Environmental sustainability, Part XA: Energy usage in buildings; 2011; South Africa. Pretoria: South African Bureau of Standards; 2011. pp. 1-17

[21] Ziuku S, Meyer E. Energy Efficient Building Integrated Photovoltaic Housing. Alice: University of Fort Hare; 2011

[22] Makaka G, Meyer EL, McPherson M. Thermal behaviour and ventilation efficiency of a low-cost passive solar energy efficient house. Renewable Energy. 2008;33:1959-1973. DOI: 10.1016/j.renene.2007.11.014

[23] Moser Y, Gijs MAM. Miniaturized flexible temperature sensor. Journal of Microelectromechanical Systems. 2007;16:1349-1354

[24] Rontronic Measurement Solutions. The Capacitive Humidity Sensor-How It Works \& Attributes of the Uncertainty bUdget. New York: Rontronic Measurement Solutions; 2016

[25] Campbell Scientific INC. HMP60 Temperature and Relative Humidity Probe. Utah: Campbell Scientific; 2010

[26] Campbell Scientific INC. Solar Radiation Shields: For Temperature/ Relative Humidity Sensors. Utah: Campbell Scientific; 2015
[27] Ruiz-Arias JA, Gueymard CA. Worldwide inter-comparison of clear-sky solar radiation models: Consensus-based review of direct and global irradiance components simulated at the earth surface. Solar Energy. 2018;168:10-29

[28] Gueymard CA. Cloud and albedo enhancement impacts on solar irradiance using high-frequency measurements from thermopile and photodiode radiometers. Part 1 : Impacts on global horizontal irradiance. Solar Energy. 2017;153:755-765. DOI: 10.1016/j.solener.2017.05.004

[29] Kipp \& Zonen. Instruction manual-Pyranometer and albedometer. Delft: Kipp \& Zonen; 2015

[30] Kerr J, Thurtell G, Tanner C. An integrating pyranometer for climatological observer stations and mesoscale networks. Journal of Applied Meteorology. 1967;6:688-694. DOI: 10.1175/1520-0450(1967)006<0688:aip fco $>2.0 . c o ; 2$

[31] Hinrichs R, Kleinbach M. Energy: Its Use and the Environment. 5th ed. New York: BROOKS/COLE; 2013

[32] Paulescu M, Paulescu E, Gravila P, Badescu V. Weather Modeling and Forecasting of PV Systems Operation. London: Springer Science \& Business Media; 2012

[33] Marzo A, Trigo M, AlonsoMontesinos J, Martínez-Durbán M, López G, Ferrada P, et al. Daily global solar radiation estimation in desert areas using daily extreme temperatures and extraterrestrial radiation. Renewable Energy. 2017;113:303-311. DOI: 10.1016/j.renene.2017.01.061

[34] Overen OK, Meyer EL, Makaka G. Perimeter walls solar heat gain, a mechanism for building design. In: 11 Built Environment Conference; 2017. 2017. pp. 680-691 
[35] Szokolay SV. Introduction to Architectural Science: The Basis of Sustainable Design. Vol. 8. 2nd ed. Oxford: Elsevier; 2008

[36] Kaşka Ö, Yumrutaş R, Orhan A. Theoretical and experimental investigation of total equivalent temperature difference (TETD) values for building walls and flat roofs in Turkey. Energy Conversion and Management. 2008;50:2818-2825. DOI: 10.1016/j.enconman.2009.06.027

[37] Kreider JF, Curtiss PS, Rabl A. Heating and Cooling of Buildings: Design for Efficiency. 2nd ed. Denver: CRC Press; 2009

[38] ASHRAE. ASHRAE Handbook: Fundamentals. Am. Soc. Heating, Refrig. Air Cond. Eng. Atlanta, 32, 2005, TC 4.7, Energy Calculations. DOI: 10.1039/c1cs15219j

[39] Robert AP. Cooling load calculation. In: ASHRAE Handbook-Fundamentals, SI. Vol. 2001. New York: ASHRAE; 2001. pp. 1-106

[40] SABS. SANS 204:2011—South African National Standard: Energy efficiency in buildings. Pretoria: South African Bureau of Standards; Vol. 1. 2011 


\title{
Social, Economic, and Environmental Impacts of Renewable Energy Resources
}

\author{
Mahesh Kumar
}

\begin{abstract}
Conventional energy source based on coal, gas, and oil are very much helpful for the improvement in the economy of a country, but on the other hand, some bad impacts of these resources in the environment have bound us to use these resources within some limit and turned our thinking toward the renewable energy resources. The social, environmental, and economical problems can be omitted by use of renewable energy sources, because these resources are considered as environmentfriendly, having no or little emission of exhaust and poisonous gases like carbon dioxide, carbon monooxide, sulfur dioxide, etc. Renewable energy is going to be an important source for power generation in near future, because we can use these resources again and again to produce useful energy. Wind power generation is considered as having lowest water consumption, lowest relative greenhouse gas emission, and most favorable social impacts. It is considered as one of the most sustainable renewable energy sources, followed by hydropower, photovoltaic, and then geothermal. As these resources are considered as clean energy resources, they can be helpful for the mitigation of greenhouse effect and global warming effect. Local employment, better health, job opportunities, job creation, consumer choice, improvement of life standard, social bonds creation, income development, demographic impacts, social bonds creation, and community development can be achieved by the proper usage of renewable energy system. Along with the outstanding advantages of these resources, some shortcomings also exist such as the variation of output due to seasonal change, which is the common thing for wind and hydroelectric power plant; hence, special design and consideration are required, which are fulfilled by the hardware and software due to the improvement in computer technology.
\end{abstract}

Keywords: conventional energy resources, social, environmental, economical, hydropower, photovoltaic, geothermal

\section{Introduction}

Renewable energy is going to be an important source for power generation in the near future, because we can use these resources again and again to produce useful energy. The energy resources are normally classified as fossil resources, renewable, and nuclear energy resources. Different renewable energy resources, like hydropower, wind, solar, biomass, ocean energy, biofuel, geothermal, etc., provide $15-20 \%$ of the total world's energy. The world is going to turn into a global village due to more requirement of energy due to fast growing population, which leads to 
the use the fossil fuels like coal, gas, and oil to fulfill the energy requirement, which creates unsustainable situations and many problems like depletion of fossil fuels, environmental and geographical conflicts, greenhouse effect, global warming, and fluctuation in fuel prices. Due to environment-friendly and less emission of gases from renewable energy, it is considered as sustainable energy; also supported for the society from each dimensions like economic, social and environmental. "Approximately 1.6 billion people have no access to electricity and about 1.1 billion are without water supply" [1]. Renewable energy resources have an ability to complete the world's energy demand, protect the environment, and provide energy security. Along with the outstanding advantages of these resources, some shortcomings also exist like the variation of output due to seasonal change, which is the common thing for wind and hydroelectric power plant; hence, special design and consideration are required, which are fulfilled by the hardware and software due to the improvement in computer technology. The main renewable energy sources with their usage in different form are classified in Table 1, and it is expected that renewable energy will be one of the important sources for the future; the world's renewable energy sources scenario by 2040 is estimated as given in Table 2.

\begin{tabular}{ll}
\hline Energy resource & Energy conversion and usage option \\
\hline Hydropower & Power generation \\
\hline Biomass & Heat and power generation, pyrolysis, gasification, digestion \\
\hline Geothermal & Urban heating, power generation, hydrothermal, hot rock \\
\hline Solar & Solar home system, solar dryers, solar cookers \\
\hline Direct solar & Photovoltaic, thermal power generation, water heaters \\
\hline Wind & Power generation, wind generators, windmills \\
\hline Wave & Numerous designs \\
\hline Tidal & Barrage, tidal stream \\
\hline
\end{tabular}

Table 1.

Main renewable energy sources with their usage form [2].

\begin{tabular}{lccccc}
\hline & $\mathbf{2 0 0 1}$ & $\mathbf{2 0 1 0}$ & $\mathbf{2 0 2 0}$ & $\mathbf{2 0 3 0}$ & $\mathbf{2 0 4 0}$ \\
\hline Total consumption (million tons equivalent) & 10,038 & 10,549 & 11,425 & 12,352 & 13,310 \\
\hline Biomass & 1080 & 1313 & 1791 & 2483 & 3271 \\
\hline Large hydro & 22.7 & 266 & 309 & 341 & 358 \\
\hline Geothermal & 43.7 & 86 & 186 & 333 & 493 \\
\hline Small hydro & 9.5 & 19 & 49 & 106 & 189 \\
\hline Wind & 4.7 & 44 & 266 & 542 & 688 \\
\hline Solar thermal & 4.1 & 15 & 66 & 244 & 480 \\
\hline Photovoltaic & 0.1 & 2 & 24 & 221 & 784 \\
\hline Solar thermal electricity & 0.1 & 0.4 & 3 & 16 & 68 \\
\hline Marine (tidal/wave/ocean) & 0.05 & 0.1 & 0.4 & 3 & 20 \\
\hline Total RES & 1365,5 & 1745,5 & 2964,4 & 4289 & 6351 \\
\hline Renewable energy contribution source (\%) & 13.6 & 16.6 & 23.6 & 34.7 & 47.7 \\
\hline
\end{tabular}

Table 2.

Global renewable energy scenario by 2040 [3]. 


\section{Background}

The economy of Pakistan has been variable and unstable for a long time, but it started to grow somehow since 1990s. Energy demand also increased, as the economy of the country increased. To fulfill the energy demand, oil, natural gas, and coal are used, but due to limited resources, Pakistan is forced to import oil and gases from U.A.E and Saudi Arabia. The location of Pakistan is very good for getting benefit from the sun to generate power, and there are also some places suitable for wind power generation in Pakistan. However, the main problem to generate power is the funding. The energy overview of Pakistan is given in Figure 1. Ref. [4] addressed the impacts of renewable energy projects (REP) on the community in Australia. The study focused on four major factors impacting REP: social, political, economic, and environmental. According to one prediction, the world's energy demand will be increased up to 5 times from that of current demand. Currently, three-fourths of that demand is fulfilled by the fossil fuels. On the other hand, the more usage of these resources causes environment pollution and results in more greenhouse effect [5]. For the protection of environment, social development and economics benefits can be get by using renewable energy sources, because there is no requirement of fuel [6]. These resources avoid the fluctuations in prices and importing of fossil fuel. Wind energy has some effects like bird strike and noise etc., which can be mitigated by proper placement of installation. The hydroelectric power may develop slowly with respect to other resources, because a number of people have to leave their homes. But, this may be beneficial for the companies to improve flood control [7]. The increasing global warming effect can easily be prevented with the proper access of renewable energy and by improving the renewable energy technologies [8]. In developing countries like Pakistan, our main focus is to create jobs and the financial development, than focusing on the environment impacts; with the shift of consumers' attention toward renewable energy, society will be more effective and efficient and enhancement in smart gird system [9].

Renewable energy source will be the best option for minimizing pollution, increasing economy, energy security, and job opportunities; also, poverty will be reduced because mostly poor people rely on the natural resources [10]. It is believed that after $2050,50 \%$ of global energy supply will be generated using renewable energy resources; the magnitude of renewable energy sources is 140 times the worldwide annual energy consumption. Renewable energy resources as "job motor for Germany," 55\% increase in total number of jobs since 2004, reported in a publication from Environmental Ministry (BMU) [11]. Pakistan has

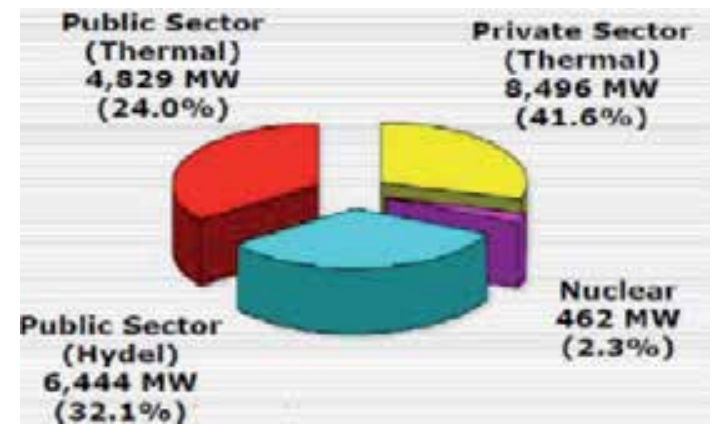

Figure 1.

Energy overview of Pakistan [22]. 
abundant renewable energy resources and also shows the potential to overcome the energy demand gap, but it is inhibited by some factors like policy, institutional, regulatory, fiscal, social, economic, technical, industrial, and informational barriers [12]. Globally, around three billion people rely on solid fuel mostly fossil fuel, causing health concerns and diseases like pneumonia, chronic respiratory diseases, and lung cancer. It is found that with the $1 \%$ increment of growth there will be an increment in $\mathrm{CO}_{2}$ emission up to $0.84 \%$ [13]. Population and GDP per capita have positive impacts on increasing $\mathrm{CO}_{2}$ emission. Government of Pakistan should initiate, in short run, small dams in the northern area and, in long run, big dams and hydro power projects, and for domestic purposes, coal and hydel resources can be used in small scale [14]. With the proper and efficient use of energy, the culture will be developed [15]. Still most of the northern areas of Pakistan are not electrified and we are under the huge crises of electric power; urban and rural areas experienced 10-12 and 16-18 h, respectively, of load shedding, which is caused direct decrement in the overall economics. With solar cell, electricity can be generated but in small amount, which would be useful as backup during load shedding time. In Baluchistan, there is no scope of gird system because of scattered villages; $77 \%$ of the population lives in villages and $90 \%$ of them do not have electricity [16]. In Pakistan, big cities produce millions of tons of biomass, but lack of technologies to generate electric power from these wastes is one of the biggest barriers for the improvement in renewable energy resources in Pakistan. Pakistan has potential to produce almost 652 million $\mathrm{kg}$ of manure per day, only from cattle and buffalo. It can produce 16.6 million $\mathrm{m}^{3}$ biogas daily, and 21 million tons of biofertilizer can be generated per year. That means $20 \%$ nitrogen and $66 \%$ phosphorous can be provided to the crop fields. Additionally, $3000 \mathrm{MW}$ energy can be generated from sugarcane industries. A $10 \mathrm{~m}^{3}$ biogas unit can save almost 92,062 PKR per year. Finally, the study concluded that biogas energy system has low initial cost, low operating cost, and positive impact on household income. Biogas energy can do good for almost 70\% of the country's population living in rural areas [17]. Nuclear energy can be useful for the development in the long term to meet global increasing demand [18].

Every year, Pakistan spends 3 billion US dollars to import oil to meet the energy requirement, and this ratio is increasing $1 \%$ yearly. Decreased efficiencies of thermal plants, periodic changes in water flow, fuel availability, auxiliary consumption and transmission limitations are main cause that Maximum system capability is lower. The main cause of load shedding is the circular debt caused by government institutions, poor revenue collection, insufficient tariff, corruption, losses, theft of electric power, and dispute on tariff with FATA, AJK, and KESC and also due to ignorance of merit, appointments of noneligible employees on political basis, etc. Circular debt can only be improved with the introduction of more and more renewable energy to the national gird [19]. Nonrenewable consumption increases the real GDP rapidly as compared to renewable energy consumption. However, it has $87 \%$ variation in carbon dioxide emission, which causes deforestation and dangerous impacts on the human health and the environment. Finally, it was concluded that renewable energy consumption along with nonrenewable energy consumption is the better solution for the GDP growth of the country [20]. It is found that economical, technical, reliability, availability of renewable energy resources, and financial risk are the important factors for selection and ranking of renewable energy technologies. The study prioritizes the renewable energy resources as wind energy, biomass, solar photovoltaic, and solar thermal energy. Further, wind energy and biomass were preferred for power generation in Pakistan [21], and energy review of Pakistan is shown in Figure 1. 


\section{Impacts of renewable energy resources}

\subsection{Social impacts}

These resources also provide social benefits like improvement of health, according to choice of consumer, advancement in technologies, and opportunities for the work, but some basic considerations should be taken for the benefit of humans, for example, climate conditions, level of education and standard of living, and region whether urban or rural from agricultural point of view. Social aspects are the basic considerations for the development of any country. The following social benefits can be achieved by renewable energy systems: local employment, better health, job opportunities, and consumer choice. The study concluded that the total emission reduction is exponentially increasing in different years after the installation of renewable energy projects in remote areas [23]. Social impacts of each resource with its magnitude are listed in Table 3.

\subsection{Economics}

It was discovered that renewable energy projects provide benefits in economic point of view because they utilize local labor from rural areas, local material and business, local shareholders, and services of local banks. In addition, the renewable energy projects have facilitated the communities by establishing a trust fund that aims to invest the money earned by selling electricity in local economy. This makes it easy for a few communities to invest money on any small business of their own choice [4]. Biofuel projects created large number of jobs; however, very low jobs were created by solar power plants, as the ratio of people working in different companies increase that will create more jobs for others by using the part of their economy for entertainment, leisure, restaurant, etc. The consumers will be provided with electric power at a low cost as compared to that of conventional energy sources, and overall economy will be enhanced because there will be multiple options to generate power using different renewable energy sources present in that region [23].

\begin{tabular}{lll}
\hline Technology & Impact & Magnitude \\
\hline \multirow{2}{*}{ Photovoltaic } & Toxins & Minor-Major \\
\cline { 2 - 3 } & Visual & Minor \\
\hline \multirow{2}{*}{ Wind } & Bird strike & Minor \\
\cline { 2 - 3 } & Noise & Minor \\
\cline { 2 - 3 } & Visual & Minor \\
\hline \multirow{2}{*}{ Hydro } & Displacement & Minor-Major \\
\cline { 2 - 3 } & Agricultural & Minor-Major \\
\cline { 2 - 3 } & River damage & Minor-Major \\
\hline \multirow{2}{*}{ Geothermal } & Seismic activity & Minor \\
\cline { 2 - 3 } & Odor & Minor \\
\cline { 2 - 3 } & Pollution & Minor-Major \\
\cline { 2 - 3 } & Noise & Minor \\
\hline
\end{tabular}

Table 3.

Social impacts assessment for different renewable energy sources [7]. 


\subsection{Environmental impacts}

Renewable energy projects have also contributed in improving environmental impacts such as reduction of carbon dioxide gas, awakening community about the climate change. The study observed very small impacts on the people living in a particular area, tourism, cost of energy supply, and educational impacts. Significant impacts were observed in improvement of life standard, social bonds creation, and community development. They also observed that the renewable energy projects are complex to install and are local environmental and condition sensitive. Their forecasting, execution, and planning require more consideration and knowledge as compared to other projects [4]. The two main aspects of environment are air and water pollution, normally created by the discharged water from houses, industries, and polluted rain, and discharge of used oils and liquids contains poisonous chemicals and heavy metals like mercury, lead, etc. Along with water pollution, natural resources can be maintained and greenhouse effect and air pollution can be mitigated by the proper usage of renewable energy sources [23] as shown in Table 4. Carbon dioxide emission with the generation of electric power using different energy resources is given in Figure 2.

Various greenhouse gases in atmosphere is being increased by humankind by doing many economic activities. The role of greenhouse gases and current situation are given in Table 5 .

\subsection{Sociopolitical impacts}

Solar panels are usually installed at the roofs of the buildings that increase the job opportunities in the PV system fabrication and installation. This increases the regional development and reduces the usage of energy from nonrenewable energy projects. It is very useful at the regions where there is no access of electricity. The major problem

\begin{tabular}{lll}
\hline Category of impact & $\begin{array}{l}\text { Relationship to } \\
\text { conventional sources }\end{array}$ & Comment \\
\hline $\begin{array}{l}\text { Exposure to harmful } \\
\text { chemicals }\end{array}$ & Reduced emissions & $\begin{array}{l}\text { Emission reduced a few hundred } \\
\text { times. }\end{array}$ \\
\hline toxic elements & Reduced emissions & Much less emission. \\
\hline Emission of particles & Exposure to harmful gases & \\
\hline $\mathrm{CO}_{2}$ emission & Reduced emissions & A big advantage. \\
\hline Acid rain, SO, NO $\mathrm{x}_{\mathrm{n}}$ & Reduced emissions & Reduced more than 25 times. \\
\hline Other greenhouse gases & Reduced greenhouse gases & Big advantage-global warming. \\
\hline Spouts off fossil fuels & Other & $\begin{array}{l}\text { Heavy fuel oil and other petroleum } \\
\text { product spills. }\end{array}$ \\
\hline Water quality & Better quality water & Reduced water pollution. \\
\hline Soil erosion & Smaller loss of land & $\begin{array}{l}\text { In most cases, there is no penetration } \\
\text { deep into earth. }\end{array}$ \\
\hline
\end{tabular}

Table 4.

Summary of environmental effects [24]. 


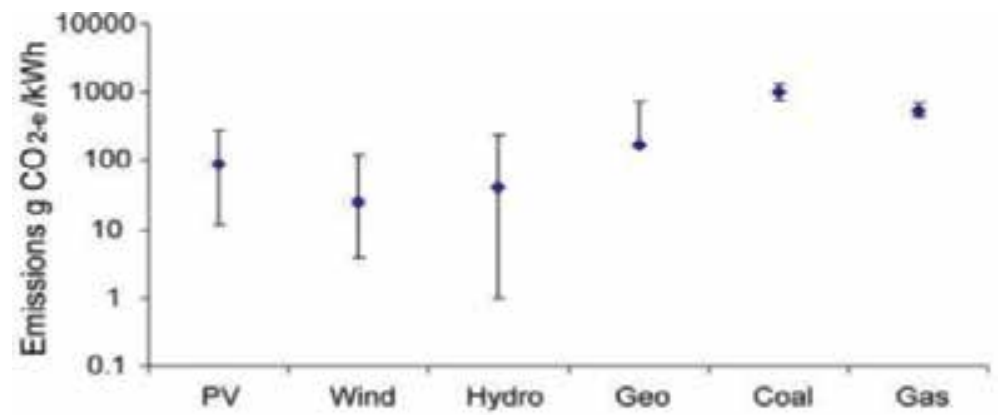

Figure 2.

Carbon dioxide equivalent emission during power generation [7].

\begin{tabular}{lcccccc}
\hline Substance & $\begin{array}{c}\text { Ability } \\
\text { to retain } \\
\text { infrared } \\
\text { radiation } \\
\text { compared } \\
\text { to CO } 2\end{array}$ & $\begin{array}{c}\text { Preindustrial } \\
\text { concentration }\end{array}$ & $\begin{array}{c}\text { Present } \\
\text { concentration }\end{array}$ & $\begin{array}{c}\text { Annual } \\
\text { growth } \\
\text { rate } \\
(\%)\end{array}$ & $\begin{array}{c}\text { Share in } \\
\text { greenhouse } \\
\text { effect due } \\
\text { to human } \\
\text { activity }\end{array}$ & $\begin{array}{c}\text { Share in } \\
\text { greenhouse } \\
\text { effect increase } \\
\text { due to human } \\
\text { activity }\end{array}$ \\
\hline Alpha & 1 & 275 & 346 & 0.4 & 71 & $50 \pm 5$ \\
\hline Beta & 25 & 0.75 & 1.65 & 1.0 & 8 & $15 \pm 5$ \\
\hline Gamma & 250 & 0.25 & 0.35 & 0.2 & 18 & $9 \pm 5$ \\
\hline Delta & 17,500 & 0 & 0.00023 & 5.0 & 1 & $13 \pm 5$ \\
\hline Epsilon & 20,000 & 0 & 0.00040 & 5.0 & 2 & $13 \pm 5$ \\
\hline
\end{tabular}

Table 5 .

Role of different substances in greenhouse effect [15].

with solar system is the high investment and maintenance cost. Biomass energy projects have great contribution in the local job creation and the development of rural areas. Such types of power plants have large opportunities of jobs in construction of plants, management, maintenance of plants, production, and preparation of biomass. Only the noise production and unpleasant smell are the negative impacts of these plants. Fuel cells have slow implementation because of their high cost of plant construction and energy generation. Their construction and operation create jobs in almost all technical activities. In hydro power plants, the major sociopolitical problem is the shifting of the people from the areas where the plant is going to be constructed. These plants provide significant jobs for local community and also play an important role in the economic development of the community. The construction of tidal energy plants has no effect on humans, and they have better contribution in the local and official employment. These plants are very expensive and are not common. Wind energy projects do not have any emigration problem, and they create large number of job opportunities especially for engineers. Geo thermal energy projects provide the following sociopolitical benefits: improvement in the education of local people, improvement in living standards, and improvement in the care of health issues [25].

\subsection{Impacts on grid}

When the solar panels are connected to the distribution system, the cost of safety equipment is reduced because their short circuit current is higher than the nominal value. Biomass power plants have the same effects on the gird as do conventional plants. The integration of wind energy plants, tidal energy, and geothermal energy is complex [25]. 


\subsection{Socioeconomic impacts}

Three case studies were made to investigate the socioeconomic benefits of renewable energy projects, and the three cases were solar, wind, and biofuel energy projects; empirical method was used to collect data. The basic aim of study was to know the contribution of renewable energy projects to local sustainability, which includes social, economic, and environmental, and to identify the socioeconomic benefits of REPs through the concerned community. It was done by doing survey of the communities. Eleven parameters were used including job creation, impacts on education, easy usage of energy, income development, demographic impacts, social bonds creation and community development, usage of native resources, and tourism. They concluded that the impacts of REPs on employment are positive, and indirect employment is high in comparison with the size of community, whereas direct employment is moderate [26].

\section{Availability and technical limitations}

One of the important assessing factors to generate power from renewable energy sources is the availability and their technical limitation. Each resource has some limitations; photovoltaic has limitation to generate power only because heat energy from sun can only be received during the day time, except cloudy season. For wind turbine, speed should not increase beyond $25 \mathrm{~m} / \mathrm{s}$; otherwise, turbine will be damaged. Also, low speed of wind, that is, $<3 \mathrm{~m} / \mathrm{s}$, will not be sufficient for the generation of electric power. Geothermal has good ability to generate power throughout the day for $24 \mathrm{~h}$ but is geography limited according to the presence of resources. Hydro-electric power plants are easy to start, stop, and operate within minutes; hence, they are considered as one of the highest available, reliable, and flexible renewable energy resources. From efficiency point of view, hydroelectric is classified at the top of the list, and then wind energy, photovoltaic, and geothermal are lowest efficient renewable energy resources. Because of availability of cells in different categories, the efficiency of photovoltaic is very much variable [7]. According to the efficiency, different energy sources are categorized in Table 6.

\begin{tabular}{lc}
\hline Technique & Efficiency \\
\hline Photovoltaic & $4-22 \%$ \\
\hline Wind & $24-54 \%$ \\
\hline Hydro & $>90 \%$ \\
\hline Geothermal & $10-20 \%$ \\
\hline Coal & $32-45 \%$ \\
\hline Gas & $45-53 \%$ \\
\hline
\end{tabular}

Table 6.

Efficiency of electricity generation [7].

\section{Conclusion}

The conventional energy resources like oil, gas, and coal are very important for the improvement in economics of a country. A country like Pakistan is fully dependent on the conventional energy sources in spite of knowing its bad effects for health and environment like greenhouse effect, global warming effect, etc. Pakistan 
is blessed with all the renewable energy sources like hydro, wind, and geothermal, and for solar power generation also, it is a suitable country. But, the main problems to generate power from renewable energy resources are funds and politics. All the factors like emission of greenhouse gases, availability of resources, land requirements, water consumption, social impacts, and price of power generated are taken into consideration for the classification of renewable energy sources. Wind power generation is considered as lowest water consumption, lowest relative greenhouse gas emission, and most favorable social impacts. It is considered as one of the most sustainable renewable energy sources, followed by hydropower, photovoltaic, and then geothermal. Biomass is considered suitable for the small-scale industries because of saving of fuel in considerable amount. Local employment, better health, job opportunities, job creation, consumer choice, improvement of life standard, social bonds creation, income development, demographic impacts, social bonds creation, and community development can be achieved by the proper usage of renewable energy system. Along with benefits of renewable energy resources, these are complex to install and are local environmental and conditions sensitive. Their forecasting, execution, and planning require more consideration and knowledge as compared to other projects.

\section{Acknowledgements}

The author thanks the Mehran University of Engineering and Technology, Jamshoro, for providing the necessary facilities for carrying out this research.

\section{Author details}

\section{Mahesh Kumar}

Department of Electrical Engineering, Mehran University of Engineering and Technology, Jamshoro, Sindh, Pakistan

*Address all correspondence to: rathii.mahesh@faculty.muet.edu.pk

\section{IntechOpen}

(C) 2020 The Author(s). Licensee IntechOpen. This chapter is distributed under the terms of the Creative Commons Attribution License (http://creativecommons.org/licenses/ by/3.0), which permits unrestricted use, distribution, and reproduction in any medium, provided the original work is properly cited. (cc) BY 


\section{References}

[1] Yuksel I et al. Hydro energy and environmental policies in Turkey. Journal of Thermal Engineering. 2016;2(5):934-939

[2] Demirbaş A. Global renewable energy resources. Energy Sources, Part A: Recovery, Utilization, and Environmental Effects. 2006;8(28):779-792

[3] Kralova I, Sjöblom J. BiofuelsRenewable energy sources: A review. Journal of Dispersion Science and Technology. 2013;3(31):409-425

[4] Hicks et al. Community-owned renewable energy (CRE): Opportunities for Australia. Rural Society. 2011:244-255

[5] Ali S et al. Renewable and nonrenewable energy and its impact on environmental quality in South Asian countries. Forman Journal of Economic Studies. 2017;13:177-194

[6] Chilán JCH et al. Social impact of renewable energy sources in the province of Loja: Ecuador. International Journal of Physical Sciences and Engineering. 2018;2(1):13-25

[7] Evans A, Strezov V, Evans YJ. Renewable and Sustainable Energy Reviews. 2009;13:1082-1088

[8] Bilgil F, Kocak E, Bulut U. The dynamic impact of renewable energy consumption on $\mathrm{CO}_{2}$ emissions: $\mathrm{A}$ revisited environmental Kuznets curve approach. Renewable and Sustainable Energy Reviews. 2016;54:838-845

[9] Shahzad SJ et al. Carbon emission, energy consumption, trade openness and financial development in Pakistan: A revisit. Renewable and Sustainable Energy Reviews. 2017;70:185-192

[10] Zeb R et al. Causal links between renewable energy, environmental degradation and economic growth in selected SAARC countries. Renewable Energy. 2014;71:123-132

[11] Frondel M et al. Economic impacts from the promotion of renewable energy technologies: The German experience. Energy Policy. 2010;38:4048-4056

[12] Yazdanie M. Renewable Energy in Pakistan: Policy Strengths, Challenges $\&$ the Path Forward; June 2010

[13] Aslam S, Fatima A, Butt MS. Sustainable development in Pakistan in the context of energy consumption demand and environmental degradation. Journal of Asian Economics. 2007;18:825-837

[14] Haseeb M, Azam M. Energy consumption, economic growth and $\mathrm{CO}_{2}$ emission Nexus in Pakistan. Asian Journal of Applied Sciences. 2015

[15] Shove E, Walker G. What Is Energy For? Social Practice and Energy

Demand. 2014. pp. 1-18

[16] Shahbaz M, Zeshan M, Afza T. Is energy consumption effective to spur economic growth in Pakistan? New evidence from bounds test to level relationships and Granger causality tests. Economic Modelling. 2012;29:2310-2319

[17] Amjid SS, Bilal MQ, Nazir MS, Hussain A. Biogas, renewable energy resource for Pakistan. Renewable and Sustainable Energy Reviews. 2011;15:2833-2837

[18] Mirza UK, Ahmad N, Majeed T. An overview of biomass energy utilization in Pakistan. Renewable and Sustainable Energy Reviews. 2008;12:1988-1996

[19] Mahmood A et al. Pakistan's overall energy potential assessment, 
comparison of LNG, TAPI and IPI gas projects. Renewable and Sustainable Energy Reviews. 2014;31:182-193

[20] Shabir Muhammad S, et al. Renewable and non renewable energy consumption, real GDP, and $\mathrm{CO}_{2}$ emission Nexus. Lahore; 2011

[21] Umer M, Daim TU. Selection of renewable energy technologies for a developing county: A case of Pakistan. Energy for Sustainable Development. 2011;15:420-435

[22] Shahzad U. The importance of renewable energy sources in Pakistan. Durreesmin Journal. 2015;1(3)

[23] Akella AK et al. Social, economical and environmental impacts of renewable energy systems. Renewable Energy. 2009;34:390-396

[24] Turney VFD. Environmental impacts from the installation and operation of large scale solar power plants. Renewable and Sustainable Energy. 2011;15(6):3261-3270

[25] Vezmar S et al. Positive and negative impacts of renewable energy sources. 2014. p. 5

[26] Rio D et al. An empirical analysis of impact of renewable energy deployment on local sustainability. Renewable and Sustainable Energy Reviews.

2009:1314-1324 
